Monika Hanswillemenke, Bernd Rahmann

\title{
Zwischen Reformen \\ und Verantwortung für Vollbeschäftigung
}

Die Finanz- und Haushaltspolitik der sozialliberalen Koalition von 1969 bis 1982 


\section{Zwischen Reformen und Verantwortung für Vollbeschäftigung}

Der Beginn eines nennenswerten Anstiegs der öffentlichen Verschuldung fällt in die Zeit der sozial-liberalen Koalition von 1969 bis 1982, die zugleich eine Politik der inneren Reformen propagierte. Mitte der 70er Jahre wird diese Politik durch einen anhaltenden Beschäftigungseinbruch durchkreuzt. Daraus entsteht ein Konflikt zwischen Reformen und Verantwortung für Vollbeschäftigung. Die haushalts- und finanzpolitischen Konsequenzen zeigen sich in einer veränderten Budgetstruktur, vor allem aber in einer erhöhten öffentlichen Verschuldung. Auf der Basis des Konzepts der Tragfähigkeit öffentlicher Verschuldung wird der Zeitraum von 1969 bis 1982 analysiert und die Folgen für zukünftige Haushaltsjahre untersucht.

Monika Hanswillemenke, geboren 1966, Studium der Volkswirtschaftslehre in Paderborn und Dublin. Diplom (1993) in Paderborn. Seit 1995 dort wissenschaftliche Mitarbeiterin am Lehrstuhl für Finanzwissenschaft. Bernd Rahmann, geboren 1940, Studium der Volkswirtschaftslehre in Köln und London. Diplom (1968), Promotion (1972) und Habilitation (1979) in Köln. Seit 1980 Professor für Volkswirtschaftslehre und Finanzwissenschaft in Paderborn. 


\section{Zwischen Reformen und Verantwortung}

für Vollbeschäftigung 


\section{FINANZWISSENSCHAFTLICHE SCHRIFTEN}

Herausgegeben von den Professoren

Albers, Krause-Junk, Littmann, Oberhauser, Pohmer, Schmidt

Band 83

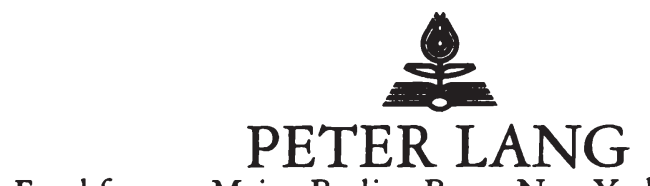

Frankfurt am Main · Berlin · Bern · New York · Paris · Wien 


\title{
Monika Hanswillemenke Bernd Rahmann
}

\author{
Zwischen Reformen \\ und Verantwortung für \\ Vollbeschäftigung
}

Die Finanz- und Haushaltspolitik der sozial-liberalen Koalition von 1969 bis 1982

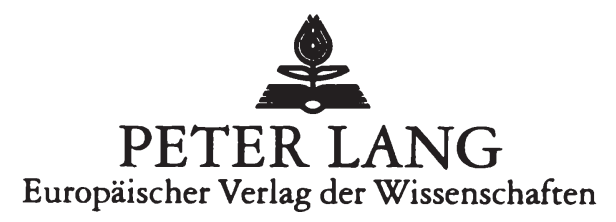


Die Deutsche Bibliothek - CIP-Einheitsaufnahme

Hanswillemenke, Monika:

Zwischen Reformen und Verantwortung für Vollbeschäftigung : die Finanz- und Haushaltspolitik der sozial-liberalen Koalition von 1969 bis 1982 / Monika Hanswillemenke ; Bernd Rahmann. - Frankfurt am Main ; Berlin ; Bern ; New York ; Paris ; Wien : Lang, 1997

(Finanzwissenschaftliche Schriften ; Bd. 83)

ISBN 3-631-32558-4

Open Access: The online version of this publication is published on www.peterlang.com and www.econstor.eu under the international Creative Commons License CC-BY 4.0. Learn more on how you can use and share this work: http://creativecommons. org/licenses/by/4.0.

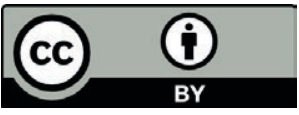

This book is available Open Access thanks to the kind support of ZBW - Leibniz-Informationszentrum Wirtschaft.

Gefördert durch die Friedrich-Ebert-Stiftung

\author{
ISSN 0170-8252 \\ ISBN 3-631-32558-4 \\ ISBN 978-3-631-75268-5 (eBook) \\ (C) Peter Lang GmbH \\ Europäischer Verlag der Wissenschaften \\ Frankfurt am Main 1997 \\ Alle Rechte vorbehalten.
}

Das Werk einschließlich aller seiner Teile ist urheberrechtlich geschützt. Jede Verwertung außerhalb der engen Grenzen des

Urheberrechtsgesetzes ist ohne Zustimmung des Verlages

unzulässig und strafbar. Das gilt insbesondere für

Vervielfältigungen, Übersetzungen, Mikroverfilmungen und die Einspeicherung und Verarbeitung in elektronischen Systemen.

Printed in Germany 123467 


\section{Inhalt}

Einleitung

1 Finanzpolitische Konzeption und Entwicklung in der sozial-liberalen Koalition

1.1 Die institutionelle Ausgangssituation

1.2 Die Haushalts- und Finanzpolitik vor und nach der Rezession 1975

1.3 Auswirkungen der Finanzpolitik auf die Defizit- und Schuldenentwicklung

1.4 Verschiebungen in der Struktur des Haushalts 47

1.4.1 Entwicklung der Ausgabenstruktur 47

1.4.2 Entwicklung der Einnahmenstruktur 53

2 Beschäftigungspolitik der sozial-liberalen Koalition 57

$\begin{array}{lll}2.1 & \text { Vorbemerkung } & 57\end{array}$

2.2 Die beschäftigungspolitische Konzeption 59

2.3 Die Entwicklung der Arbeitslosigkeit 61

2.4 Klassische oder keynesianische Arbeitslosigkeit? 66

2.5 Ein erweiterter Erklärungsansatz zur Entwicklung der Arbeitslosigkeit

2.6 Die Beschäftigungspolitik vor dem Hintergrund der Erkärungsansätze

3 Beurteilung des Deficit Spending in der sozial-liberalen Koalition aus langfristiger Sicht

3.1 Die Entwicklung der Schuldenindikatoren $\quad 89$

3.2 Zur Dynamik der Verschuldung 93

3.2.1 Zins-Wachstums-Differentiale 93

$\begin{array}{ll}3.2 .2 & \text { Finanzierungslücken } \\ & 104\end{array}$ 
3.3 Zur Tragfähigkeit der Verschuldungspolitik

3.3.1 Die Grundlagen

3.3.2 Die Entwicklung der Einnahmen- und Ausgabenlücken

3.3.3 Die Verschuldungspolitik im Kontext vorhergehender und nachfolgender Jahre

3.3.4 Auswirkungen des Haushaltsgebarens auf die Entwicklung der Indikatoren

3.3.5 Tragfähigkeit der mittelfristigen Finanzplanung

3.3.6 Die Haushaltspolitik des Bundes im Rahmen des Gesamthaushalts

3.4 Einfluß der Finanzpolitik auf Wachstum und Zinsen und ihre Auswirkungen auf die Tragfähigkeit der Verschuldung $\quad 148$

3.5 Geldpolitische Aspekte der Verschuldung 


\section{Einleitung}

Die öffentliche Diskussion über den Beitritt zur Europäischen Währungsunion hat in der Bundesrepublik Deutschland insbesondere die Staatsverschuldung in den Vordergrund des politischen Interesses gerückt. Bei der Betrachtung ihrer Entwicklung zunächst für die zurückliegenden 14 Jahre der CDU-FDP-Koalition im Bund wird ab 1982 ein Muster erkennbar, das mit Hilfe des Indikators „Schuldenquote“ (Schuldenstand in v.H. des Bruttosozialprodukts) gut veranschaulicht werden kann und das deutlich durch einen Niveauverschiebungseffekt gekennzeichnet ist.

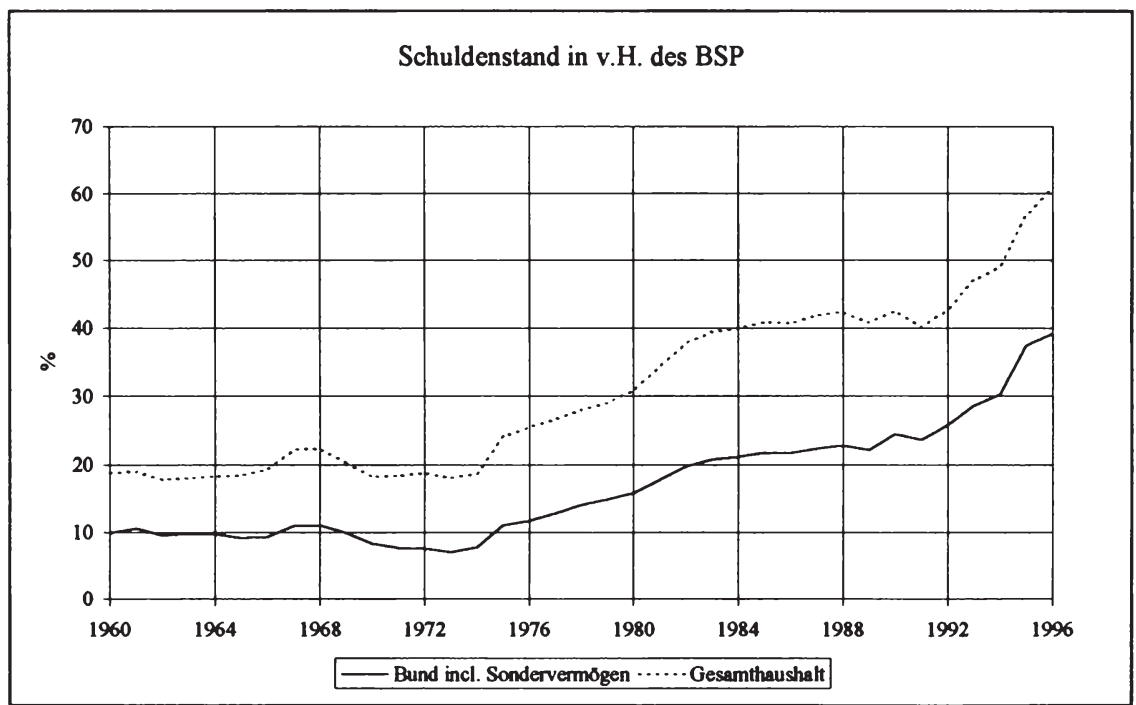

Quelle: Statistisches Bundesamt (1995), Deutsche Bundesbank (1997), Sachverstăndigenrat zur Begutachtung der gesamtwirtschaftlichen Entwicklung (1996); eigene Berechnungen.

Öffentliche Aufmerksamkeit und Problembewußtsein sind dadurch geschärft worden, daß seit 1991 die Verschuldung des Bundes einschließlich der Sonderfonds etwa so stark (und die der Gebietskörperschaften insgesamt nur wenig langsamer) gestiegen ist wie in der gesamten Zeitspanne davor seit 1960, d.h. seit dem ersten Haushaltsjahr der voll entwickelten Volkswirtschaft nach der endgültigen Überwindung der Wiederaufbauphase nach dem 2. Weltkrieg. 
Im wesentlichen sind der Niveauverschiebungseffekt und die anschließende rasante Beschleunigung der öffentlichen Verschuldung bekanntlich auf die Wiedervereinigung Deutschlands zurückzuführen. Davor vollzog sich seit Regierungsantritt im Jahre 1982 eine annähernd regelmäßige, im Trend durch Konstanz gekennzeichnete Entwicklung. Die fortgesetzte Steigerung in den 90er Jahren ist - rein statistisch betrachtet - hauptsächlich auf die der Wiedervereinigung zuzurechnenden Sonderfonds konzentriert; sie muß politisch bewertet - allerdings unzweifelhaft dem Bund zugerechnet werden.

Dieses Entwicklungsmuster ist nicht einmalig, sondern es wiederholt sich in der Bundesrepublik zum zweiten Mal. Wie aus der Abbildung klar ersichtlich, ist auch die Periode von 1960 bis Anfang der 80er Jahre durch eine zunächst trendmäßig konstante und nur durch die 1967er Rezession konjunkturell vorübergehend unterbrochene, Mitte der 70er Jahre dann aber sprunghaft und nachhaltig veränderte und weiter steigende Schuldenquote charakterisierbar. Im Gegensatz zu 1967 wird die rezessionsbedingte Veränderung des Jahres 1975 nicht wieder ausgeglichen. Eingebettet in diese Entwicklung ist die Regierungsperiode der sozial-liberalen Koalition im Bund von 1969 bis 1982. Der Niveauverschiebungseffekt ist auf die schwere Arbeitsmarktkrise, den Ölpreisschock sowie auf verschärfte Verteilungsauseinandersetzungen im öffentlichen Dienst Mitte der 70er Jahre zurückzuführen. In der Folgezeit kämpft die Politik mit den Auswirkungen der Krise, und dies ist begleitet von einer weiteren Steigerung der Staatsverschuldung. Wie seinerzeit der Konflikt für die sozial-liberale Regierung zwischen Reformeifer und Verantwortung für die Wiedergewinnung der gesamtwirtschaftlichen Vollbeschäftigung die Politik beherrschte, kann gegenwärtig beobachtet werden, wie für die christlich-liberale Koalition der Konflikt zwischen finanzpolitischem Konsolidierungseifer und Kampf mit den wirtschaftlichen Folgen der Wiedervereinigung zum Desaster für die Staatsverschuldung wird.

Die skizzierte Parallelität der Entwicklungen legt für uns den Versuch nahe, die Finanz- und Haushaltspolitik der sozial-liberalen Koalition von 1969 bis 1982 noch einmal insgesamt zu untersuchen und dabei insbesondere die Frage nach der öffentlichen Verschuldung zu stellen in der Hoffnung, aus der jüngeren Geschichte verwertbare Erkenntnisse für die Gegenwart gewinnen zu können. Bei der in der folgenden Arbeit versuchten Beantwortung dieser Frage im Rahmen einer ökonomischen Analyse stehen mittlerweile 
differenziertere Methoden zur Bewertung der öffentlichen Verschuldung im gesamtwirtschaftlichen Kontext zur Verfügung. Dabei wird die Frage nach der Tragfähigkeit einer volkswirtschaftlichen Entwicklung mit öffentlicher Verschuldung aufgeworfen. Die Antwort darauf wird vor dem Hintergrund einer theoretisch fundierten und modellhaft skizzierten Untersuchung der Bedingungen einer dauerhaften Existenz eines gesamtwirtschaftlichen Systems mit defizitärem Staatsbudget formuliert. Die allgemeine Problemstellung ist im Prinzip sehr alt, wie die theoretische Diskussion der Rechtfertigungsargumente für öffentliche Verschuldung in der finanzwissenschaftlichen Literatur belegt. ${ }^{1}$ Sie politisch konkret auf eine abgeschlossene, zurückliegende Regierungsperiode zu projizieren, hat aber nun den unschätzbaren Vorteil der definitiven zeitlichen Begrenzung für die Zuordnung haushalts- und finanzpolitischer Entscheidungen und ihrer politischen Zurechenbarkeit zur zeitgleich beobachteten gesamtwirtschaftlichen Entwicklung. Deshalb ist unser analytisches Interesse auf die Haushalts- und Finanzpolitik der sozial-liberalen Koalition gerichtet. Quasi resümierend können wir dann im Gesamtkontext auch die Frage aufwerfen, inwieweit öffentliche Verschuldung eine Last für die Zukunft darstellt dergestalt, daß eigenständige finanzpolitische Entscheidungen durch die in der Vergangenheit geschaffenen Fakten möglicherweise eingeschränkt werden. Auch hier ist wiederum die faktische Zäsur bei der politischen Regierungsverantwortung von $\mathrm{Be}$ deutung.

Unser Interesse ist auf den Bund konzentriert, um eindeutige politische Abgrenzungen nicht nur zeitlich, sondern auch parteipolitisch definieren zu können. Die Gesamtwirtschaft wird lediglich als Vergleichshintergrund für quantitative Entwicklungen betrachtet. Eine differenzierte Analyse der Bundesländer mit unterschiedlichen politischen Machtverhältnissen unterbleibt. Zudem wird die Haushalts- und Finanzpolitik unter dem spezifischen Blickwinkel der Verschuldung gesehen und insbesondere in Zusammenhang mit der volkswirtschaftlichen Stabilisierungspolitik gebracht. Dabei tritt zwangsläufig das Problem der Bekämpfung von Arbeitslosigkeit in den Vordergrund. Spezielle allokative und distributive Entscheidungen werden nicht gesondert analysiert. Es steht auch nicht die konkrete, im Zeitverlauf erfolgte politische Entscheidung persönlich identifizierter Personen zur Diskussion²,

1 Siehe im Überblick Gandenberger (1981).

2 Wie etwa bei Scherf (1986). 
sondern das in Zahlen meßbare Ergebnis der insgesamt erfolgten Politik. Es interessiert uns also, ob das quantifizierte Gesamtresultat aller tatsächlich erfolgten Entscheidungen tendenzielle Deutungen zuläßt. Bezüglich der politischen Absichten, die hinter den Aktivitäten standen, werden die regierungsseitigen Erklärungen herangezogen. Für unsere verbale Zustandsbeschreibung berücksichtigen wir die seinerzeit veröffentlichte Literatur. Damit soll der wissenschaftliche Erkenntnisstand dokumentiert werden, der für die damalige politische Entscheidungsfindung zur Verfügung stand.

Unsere Untersuchung ist in vier Kapitel untergliedert. Zunächst werden die Konturen der Haushalts- und Finanzpolitik der sozial-liberalen Bundesregierung vor und nach dem für den Niveauverschiebungseffekt entscheidenden Rezessionsjahr 1975 nachgezeichnet. Es wird gezeigt, wie die anfangs auf Reformpolitik in weiten Teilen der Gesellschaft ausgerichtete Aktivität rasch und zunehmend von für unabwendbar erachteten Arbeitsmarktprogrammen bedrängt und später sogar verdrängt wird. Im zweiten Kapitel wird die Entwicklung der gesamtwirtschaftlichen Arbeitslosigkeit von einer ursprünglich konjunkturellen zu einer verstärkt strukturell verhärteten und damit dauerhaften Erscheinung untersucht. Hier steht insbesondere die Frage nach den konkurrierenden theoretischen Erklärungsansätzen im Sinne keynesianischer oder klassischer Arbeitslosigkeit im Vordergrund, um mit dieser Unterscheidung auch die verschiedenen konjunkturell-nachfrageseitig bzw. strukturellangebotsseitig ausgerichteten Arbeitsmarktprogramme beurteilen zu können. Das dritte Kapitel ist dann der konkreten haushalts- und finanzpolitischen Situation gewidmet. Hier wird mit den faktisch und unveränderbar vorliegenden Daten gerechnet und das Ergebnis nach heutigen Erkenntnissen interpretiert. Dabei steht im Hintergrund der bereits erwähnte Aspekt der Nachhaltigkeit. Wir wenden ihn insbesondere auf die fiskalischen Konsequenzen der öffentlichen Verschuldung an, also auf den Konflikt zwischen zukünftigem Schuldendienst und konkurrierenden öffentlichen Aufgaben bzw. Ausgaben. Indem empirisch aber der gesamtwirtschaftliche Rahmen berücksichtigt wird, erfassen wir zumindest implizit auch die übrigen ökonomischen Wirkungen der öffentlichen Verschuldung. Wir entwickeln dagegen kein differenziertes Modell mit hypothetischen Funktionen endogenisierter Zusammenhänge zwischen öffentlicher Schuld und anderen Makrogrößen. Vielmehr wollen wir die praktisch vollzogene Politik und die daraus entstandenen Datenreihen für eine bestimmte Zeitperiode nach dem theoretischen 
Kriterium der fiskalischen Tragfähigkeit beurteilen. Wir sind uns der spezifischen Sichtweise durchaus bewußt. Deshalb erfolgt im vierten Kapitel eine relativierende Bewertung unserer Berechnungen, die wir zunächst formal im Rahmen der verfügbaren Daten gar nicht anders vornehmen konnten. Ein schlußfolgerndes Resümee kann dagegen die Frage aufwerfen, welche Konsequenzen eingetreten wären, wenn es eine andere als die vorgezeichnete Empirie gegeben hätte. Diese kann natürlich mangels vorfindbarer Daten nicht berechnet werden. Das Fazit stellt also der quantitativen Berechnung eine qualitative Alternative gegenüber. Für eine zurückliegende Zeit ist eine solche Gegenüberstellung selbstverständlich nicht mehr von praktischem Wert. Für die Zukunft eröffnet sich daraus dagegen die Möglichkeit zu lernen. Wir beziehen unsere Erkenntnis dabei speziell auf die eingangs erwähnte Diskussion über die Beitrittskriterien zum Maastrichter Vertrag und lernen daraus, daß eine einseitig auf die rein fiskalischen Wirkungen der öffentlichen Verschuldung gerichtete Beurteilung zu kurz greift, um die gesamtwirtschaftliche Widerstandsfähigkeit einer Volkswirtschaft und damit ihre Güteklasse zu beschreiben. Es ist allerdings hier nicht unsere Absicht, neue Beitrittskriterien zu formulieren.

Die vorliegende Arbeit ist mit freundlicher finanzieller Unterstützung der Friedrich-Ebert-Stiftung entstanden; dafür bedanken wir uns. Dank entrichten wir auch an Jochem Langkau, Alois Oberhauser, Otto Roloff und KarlHeinz Schmidt für aufmerksame Kritik und wertvolle Hinweise zu früheren Entwürfen unserer Studie. Last not least danken wir Ulrich Berhorst, Nikola Ebker, Monika Hentschel und Marko Köthenbürger für ihre Unterstützung mit Rat und Tat. Wir sind nicht allen Ratschlägen voll gefolgt und wie üblich verbleibt die Verantwortung für den veröffentlichten Text selbstverständlich bei der Verfasserin und dem Verfasser. 
Monika Hanswillemenke and Bernd Rahmann - 978-3-631-75268-5

Downloaded from PubFactory at 01/11/2019 06:29:54AM

via free access 


\section{Finanzpolitische Konzeption und Entwicklung in der sozial-liberalen Koalition}

\subsection{Die institutionelle Ausgangssituation}

Die Finanz- und Haushaltspolitik der sozial-liberalen Regierungskoalition muß vor dem Hintergrund der seinerzeit allgemein anerkannten neuen volkswirtschaftlichen bzw. gesamtwirtschaftlichen Lenkungsfunktion des öffentlichen Haushaltes gesehen werden. Diese auf der makroökonomischen keynesianischen Kreislauftheorie basierende Aufgabenzuweisung an den (Zentral-)Staat löst seit Mitte der 50er Jahre allmählich die ordoliberale Doktrin der Wirtschaftspolitik im Sinne von Walter Eucken ab und integriert Konjunkturpolitik in das Konzept der sozialen Marktwirtschaft von Alfred Müller-Armack bzw. Ludwig Erhard. ${ }^{3} 1956$ veröffentlichten die Wissenschaftlichen Beiräte beim Bundesministerium für Wirtschaft und beim Bundesministerium der Finanzen gemeinsam ein Gutachten über „Instrumente der Konjunkturpolitik und ihre rechtliche Institutionalisierung“. Darin heißt es, „daß ein stetiges Wachstum der Volkswirtschaft am besten sichergestellt wird, wenn es der Wirtschaftspolitik gelingt, die Kaufkraft der Währungseinheit... (Niveau der Konsumgüterpreise)... tunlichst stabil, die Beschäftigung der Produktionskräfte möglichst hoch und die Zahlungsbilanz... ausgeglichen zu halten. Maßnahmen, die dieser dreifachen Zielsetzung dienen, sollen hier unter dem Worte „Konjunkturpolitik“ verstanden werden. ${ }^{\text {4 }} \mathrm{Im}$ Jahre 1963 wird dann das „Gesetz über die Bildung eines Sachverständigenrates zur Begutachtung der gesamtwirtschaftlichen Entwicklung“ verabschiedet; darin wird gefordert, zu „untersuchen, wie im Rahmen der marktwirtschaftlichen Ordnung gleichzeitig Stabilität des Preisniveaus,

3 Vgl. zu paradigmatischen Phasen in der bundesrepublikanischen Wirtschaftspolitik Bombach/Netzband/ Ramser/Timmermann (1983), S. If.

4 Zitiert nach Bombach/Netzband/Ramser/Timmermann (1983), S. 70. Bemerkenswerterweise gehen die Beiräte davon aus, daß die „maximale Steigerung des realen Volkseinkommens je Kopf... ein mögliches, aber sicher nicht das einzig denkbare Ziel der Wirtschaftspolitik (ist). Vielmehr kann es zugunsten anderer, z.B. kultur-, sozial- oder auch wirtschaftspolitischer Zielsetzungen durchaus sinnvoll sein, sich mit einem Tempo... unterhalb des an sich Erreichbaren zu begnügen oder sogar die Wachstumsrate absichtlich niedriger als möglich zu halten." (ebenda TZ 1 bei I: Zielsetzung). 
hoher Beschäftigungsstand und außenwirtschaftliches Gleichgewicht bei stetigem und angemessenem Wachstum gewährleistet werden können“. Das "Gesetz zur Förderung der Stabilität und des Wachstums der Wirtschaft" (StabWG) vom 8. Juni 1967 ist dann der Höhepunkt dieser Entwicklung. ${ }^{5}$ Dort wiederholt sich im $\S 1$ bekanntlich der Text aus dem Gesetz über die Bildung eines Sachverständigenrates und bildet seitdem die Grundlage für den jährlich von der Bundesregierung im Januar vorzulegenden Jahreswirtschaftsbericht. Gesamtwirtschaftliche Konjunktur- und Wachstumspolitik ist (im Rahmen der marktwirtschaftlichen Ordnung) zur politischen Aufgabe geworden, die die vordem geltende Maxime der (Wieder-)Herstellung und Sicherung der marktwirtschaftlichen Ordnung, damit gesamtwirtschaftliche Abläufe störungsfrei stattfinden können, ablöst. Aufgrund der im Stabilitätsgesetz vorgesehenen Eingriffs-, Koordinations-, Informations- und Planungsinstrumente ist eindeutig zu erkennen, daß konjunkturpolitischer Steuerungsbedarf zur Erreichung gesamtwirtschaftlich zufriedenstellender Wachstumsraten gefordert wird. Das (ordnungspolitisch-liberale) Vertrauen bezüglich der Stabilisierungskräfte einer marktwirtschaftlich organisierten und ansonsten sich selbst überlassenen Wirtschaft wird jedenfalls durch die Beobachtung der Fortsetzung der regelmäßigen Wirtschaftsschwankungen nach Beendigung des 2 . Weltkrieges nachhaltig erschüttert. In die „Normalisierung“ der Wirtschaftsordnung (volle Konvertibilität seit 1958, Abschluß der Wiederaufbauphase seit 1960) paßt sich die „Normalisierung“ der zyklischen konjunkturellen Wechsellagen im Sinne des seit den ersten Beobachtungen von Clément Juglar (1860) seit über hundert Jahren stattfindenden Musters mittelfristiger Zyklen („Juglar-Zyklen") nahtlos ein. Gesamtwirtschaftliche Stabilitäts- und Wachstumspolitik ist somit antizyklische volkswirtschaftliche Lenkung.

Zentraler Bestandteil des Stabilitäts- und Wachstumsgesetzes ist die der ca. 8-jährigen Mittelfristigkeit der Konjunkturzyklen angepaßte „fünfjährige Finanzplanung“ ( $\S 9-11)$ mit tatsächlich 4-jähriger Vorherigkeit und damit eine um 3 Jahre längere Planungsperiode als der laufende Haushalt. Hier werden „Wechselbeziehungen $\mathrm{zu}$ der mutmaßlichen Entwicklung des gesamtwirtschaftlichen Leistungsvermögens... gegebenenfalls durch Alternativ-

5 Zur vollständigen inhaltlichen Information siehe Möller (1969) sowie Stern/Münch/ Hansmeyer (1972). 
rechnungen“ unterstellt ( $§ 9)$ und dementsprechend „mehrjährige Investitionsprogramme“..., „nach Dringlichkeit und Jahresabschnitten gegliedert“ gefordert ( $\$ 10)$. Fortzuführende bestehende sowie neue Investitionsvorhaben sind angemessen bei „einer die Ziele des $\S 1$ gefährdenden Abschwächung der allgemeinen Wirtschaftstätigkeit... zu beschleunigen, (so) daß mit ihrer Durchführung kurzfristig begonnen werden kann“". (§ 11)

In der Tat scheint das Gesetz seine erste Bewährungsprobe mit der raschen Überwindung der Rezession des Jahres 1967 glänzend zu bestehen. Im wesentlichen durch kurzfristig beschleunigte (vormals mittelfristig geplante) Durchführung der Elektrifizierung der Deutschen Bundesbahn erfolgt zumindest zeitgleich einerseits die Wiedergewinnung der Vollbeschäftigung und des angemessenen Wirtschaftswachstums sowie andererseits die Anwendung eines Lenkungsinstrumentes im Sinne der mittelfristigen Finanzplanung. Es ist daher verständlich, daß der Optimismus der sozial-liberalen Koalition bei der Übernahme der Regierungsverantwortung im Herbst 1969 bezüglich der Steuerbarkeit der Wirtschaft nicht nur politisch-normativ, sondern auch empirisch-positiv untermauert war. Die allgemein verbreitete und anerkannte Interpretation der keynesianischen makroökonomischen Kreislauflehre bildete dabei den theoretischen Rückhalt.

Zwar setzt die konzeptionelle und die pragmatische Kritik früh ein; sie findet jedoch nur zögerlich Eingang in den politischen Entscheidungsprozeß. Abgesehen von der prinzipiellen theoretischen Gegenposition des Monetarismus $^{6}$ (hier wird gegen die „keynesianische Revolution“ die „monetaristische Gegenrevolution" ins Feld geführt) braucht die vielfältige pragmatische Kritik offenbar erst ihre empirische Begründung in der real-wirtschaftlichen Erscheinung. Schon bald wird deutlich, daß das StabWG instrumentell asymmetrisch zuungunsten der kontraktiven Maßnahmen bei Bekämpfung von Boom und Inflation ausgestaltet ist. Zwar sind steuerliche Abschöpfungen und Abschreibungsvariationen vorgesehen ( $\$ 26$ und 27), deren Erlöse einer Konjunkturausgleichsrücklage $(\S 15)$ zuzuführen und so dem Kreislauf $\mathrm{zu}$ entziehen sind; es macht sich jedoch dabei eine weitere Asymmetrie,

6 Vgl. z.B. Friedman (1968); ders. (1970) und im Überblick Kalmbach (1973); siehe auch Phelps (1970) - ein wichtiges Buch, das erst später in der theoretischen Diskussion entsprechend zur Kenntnis genommen wird - sowie Felderer/Homburg (1994), insbesondere Kap. IX. 
nämlich in bezug auf die verteilungspolitische Dimension, bemerkbar. Während expansives Nachfragemanagement über steuerliche Begünstigungen vor allem der unteren und mittleren Einkommen zugleich einen maximalen Nachfrageeffekt erwarten läßt und die Sekundärverteilung tendenziell entzerrt, ist im Falle einer entsprechenden kontraktiv angelegten Inflationsbekämpfung eher mit einem Konflikt zwischen Verteilungs- und Stabilisierungsziel zu rechnen. Nach allen politischen Erfahrungen kann zwar „im Rahmen der marktwirtschaftlichen Ordnung" kein definitives Verteilungsziel vorgegeben werden. $\mathrm{Da} B$ im Rahmen des Stabilitätsgesetzes jedoch gänzlich darauf verzichtet wurde bzw. diese Flanke lediglich durch die ohne jede Verbindlichkeit vorgesehene Institution der „konzertierten Aktion der Gebietskörperschaften, Gewerkschaften und Unternehmerverbände“ (§ 3) mangelhaft abgedeckt wurde, ist ein merklicher Nachteil für die Stabilitätspolitik im Falle erforderlicher Kontraktionsmaßnahmen. ${ }^{7}$ So wird z.B. von August 1970 bis Juni 1971 ein Konjunkturzuschlag zur Bekämpfung der Inflation erhoben, der aber ab Juni 1972 zurückgezahlt wurde (was so nicht im StabWG vorgesehen ist). Diese Maßnahme war wirkungslos, weil sie wegen der Antizipation der baldigen Rückzahlung die Konsumausgaben nicht nachhaltig einschränken half. Auch eine ähnliche spätere Maßnahme von Juni 1973 bis April 1974 war - obwohl ohne vorgesehene Rückerstattung - kaum erfolgreicher und wurde zudem in darauf folgenden Verteilungskämpfen gewissermaßen erfolgreich kompensiert.

Eine weitere frühe Erkenntnis bezieht sich auf den Mangel global angelegter makroökonomischer Interventionen bei Existenz unterschiedlicher und unberechenbarer zeitlicher Verzögerung der einzelwirtschaftlichen Wirkungen. Diese mit vielschichtigen Diagnose-, Prognose-, Entscheidungs- und Wirkungsursachen behaftete Problematik führt $\mathrm{zu}$ intensiver Diskussion der schon früher im anglo-amerikanischen Sprachraum erörterten Frage nach „rules or authorities“. 8 Die Einführung wirksamer Regelmechanismen zur Behebung diesbezüglicher Schwächen der diskretionären Fiscal Policy scheitert aber nicht zuletzt an allgemeinen und im besonderen wiederum an verteilungspolitischen Problemen.

8

Vgl. stellvertretend für viele Neumark (1971). 
Der Globalsteuerung wurde weiters schon bald der Vorwurf gemacht, bei mikroökonomisch differenzierter Struktur- und Problemlage gespaltene Wirkungen hervorzubringen. So konnte je nach Höhe des Auslastungsgrades einzelner Sektoren eine expansive Maßnahme zum einen einen gewünschten positiven Beschäftigungseffekt zeitigen, zum anderen aber auch zu Inflation führen, wenn wegen der spezifischen Auslastung keine Mengen-, sondern nur noch Preiseffekte zu erwarten waren. Voll bzw. hoch ausgelastete einzelne Sektoren wurden damit zu „,bottle necks“ auf dem Pfad zu einer erfolgreichen Stabilisierung. Schlimmstenfalls blieben Mengeneffekte sogar (wegen der erwähnten Probleme) gänzlich aus oder erfolgten erst später, was dann allein zu Inflation bei ansonsten andauernder Stagnation führte (sogenannte „Stagflation“ oder „gespaltene Konjunktur"). ${ }^{9}$

Das Gegenstück zur fehlenden binnenwirtschaftlichen Strukturorientierung ist im internationalen Kontext die mangelhafte außenwirtschaftliche Absicherung. Ohne Nennung wirklich neuer Möglichkeiten wird im $\S 4$ StabWG auf „alle Möglichkeiten der internationalen Koordination“ hingewiesen und "(S)oweit dies nicht ausreicht" auf die ,zur Wahrung des außenwirtschaftlichen Gleichgewichts zur Verfügung stehenden wirtschaftspolitischen Mittel“". Alle damals bestehenden Möglichkeiten haben jedoch ihre deutliche Einschränkung durch das System fester Wechselkurse (Bretton Woods System) und die dadurch programmierte Interventionspflicht der Zentralnotenbank gefunden. Die außenwirtschaftliche Flanke wird denn auch erst nach mühevollen und letztlich vergeblichen Versuchen einer Neuordnung des internationalen Systems fester Wechselkurse („Realignment") mit der Ablösung dieses Systems durch flexible Wechselkurse im Jahre 1973 stärker gesichert. Dies führt jedoch nicht (mehr) zu einer Renaissance der Fiskalpolitik, sondern hilft vielmehr der Geldpolitik als nunmehr verantwortlicher Pfeiler der Stabilisierung zum Durchbruch. Dort findet konsequenterweise ein Übergang zu einer geldmengenorientierten Konzeption im Sinne des neuen Monetarismus statt. Im wirtschaftspolitischen Bereich ist dieser Übergang zur Dominanz des Ziels der Geldwertstabilität aber keineswegs zu beobachten. Faktisch herrscht für den Mix einer „monetary fiscal policy" vielmehr nach wie vor die postulierte Gleichrangigkeit der Ziele des StabWG vor; allerdings mit sehr wohl unterschiedlichem Zielbe-

9 Vgl. z.B. Kock/Leifert/Schmid/Stirnberg (1977); siehe auch Eckey (1978). 
wußtsein bei Bundesregierung und Zentralnotenbank, weshalb auch hier im analogen Sinne von einer Asymmetrie gesprochen werden kann.

Schließlich (auch dies allerdings erst später) wird deutlich, daß bei länger andauernder Abschwächung der allgemeinen Wirtschaftstätigkeit und demzufolge höherem Einsatz antizyklisch konzipierter Budgetressourcen deren Finanzierung zum Problem werden kann. Das einfache Bild einer Konjunkturausgleichsrücklage, in die in Boomzeiten eingezahlt, was in Rezessionszeiten benötigt wird, stimmt bei unregelmäßigen Zyklen natürlich schnell nicht mehr, und die nach Keynes zu empfehlende Politik des Deficit Spending wird dann kontra-produktiv, wenn ihr kurzfristiger Erfolg ausbleibt, weil in der (anhaltenden) Rezession mehr private Investitionen unterbleiben als antizyklische öffentliche Ausgaben getätigt werden. Der Streit um mögliche ursächliche Zusammenhänge soll hier nicht aufgerollt oder weitergeführt werden; er ist je nach (ideologischem) Standpunkt entweder längst (klar) entschieden oder dauert bis heute an. Entscheidend ist viel einfacher, daß beide Fakten - steigende kreditfinanzierte Staatsausgaben und sinkende private Investitionen - zeitgleich auftreten und so die gegenläufigen (Multiplikator-)Effekte für die Auslastung des Produktionspotentials erzeugen können, wenn bei der Nachfrage nach Finanzierung Konkurrenz zwischen dem privaten und dem öffentlichen Sektor entsteht (Hypothese vom Crowding Out).

In einer rückblickenden zusammenfassenden Würdigung wird so leicht verständlich, daß das zunächst sowohl international („der Welt bestes Stabilisierungsprogramm“) als auch national („Grundgesetz für die Wirtschaftspolitik“) gefeierte Stabilitäts- und Wachstumsgesetz in der Zeit seiner wirklichen Bewährung, d.h. bei Inflation und Massenarbeitslosigkeit, weit weniger erfolgreich eingesetzt wurde als nach der 1967er Rezession bzw. daß es (wohl angesichts der aufgezählten Schwächen) tatsächlich „gewissermaßen vergessen“ wurde. $^{10}$ Die eher negative Einschätzung war bei Beginn der sozial-liberalen Regierungszeit jedoch keineswegs „common sense“; vielmehr galt das von Milton Friedman 1970 selbst formulierte „We are all Keynesi-

10 So Hansmeyer (1977), S. 607. Es findet sich allerdings (später) durchaus auch die Einschätzung eines ,heute oft zu unrecht unterschätzten bzw. 'vergessenen”“ Gesetzes - so bei Rürup/Körner (1985); (in der 1. Auflage 1981 fehlt dieser Passus freilich). 
ans now" nur halb ironisch und zur anderen Hälfte auch anerkennend - jedenfalls bezüglich der kurzfristig zu erwartenden Stabilisierungswirkungen keynesianischer Nachfragesteuerung. ${ }^{11}$

Der lenkungspolitische Optimismus gründet freilich nicht nur auf dem Stabilitäts- und Wachstumsgesetz, sondern auch auf einer Reihe von anderen institutionellen Vorkehrungen, die teils im Zuge dieses Gesetzes nachfolgen, teils eigenständige Ergänzungen in bezug auf die volkswirtschaftliche Lenkungsfunktion darstellen. Eindeutig im Zusammenhang mit der Verabschiedung des StabWG stehen die später im Jahre 1969 noch von der großen Koalition beschlossenen Änderungen des Grundgesetzes im Abschnitt X. über „Das Finanzwesen“. Dort wird die Formel „zur Abwehr einer Störung des gesamtwirtschaftlichen Gleichgewichts" bzw. „Erfordernisse des gesamtwirtschaftlichen Gleichgewichts" an mehreren Stellen aufgenommen: bei der Lastverteilung (neuer Art. 104a), der Haushaltswirtschaft (Art. 109) und der Kreditbeschaffung (Art. 115). Insbesondere die verfassungsgerechten Regeln zur „Aufnahme von Krediten sowie die Übernahme von Bürgschaften, Garantien oder sonstigen Gewährleistungen, die zu Ausgaben in künttigen Rechnungsjahren führen können“, erfahren gewissermaßen einen Paradigmawechsel: Die bisherige objektorientierte öffentliche Verschuldung, deren Begründung mit der Lehre von Adolph Wagner in das späte 19. Jahrhundert zurückging, wird abgelöst durch die sogenannte ,situationsorientierte Verschuldung", deren Begründung eben auf dem vermuteten Erfolg des Deficit Spending im Sinne von J.M. Keynes basiert. Nicht mehr die (nur schwer bis gar nicht meßbaren und zurechenbaren "werbenden Zwecke", d.h. die) Ertragsfähigkeit einzelner Objekte, aus der der fällige Schuldendienst und die Tilgung zu finanzieren waren, sondern die insgesamt verstärkte Wachstumsaktivität der Volkswirtschaft im Zuge der erfolgreichen „Abwehr einer Störung des gesamtwirtschaftlichen Gleichgewichts" soll die aus öffentlicher Verschuldung resultierenden zukünftigen Lasten abtragen helfen. Und dies gelingt natürlich auch, wenn das „Objekt“ Gesamtwirtschaft die gewünschten Wachstumsraten hervorbringt und die daran anknüpfenden Steuermehreinnahmen tatsächlich für Schuldendienst und Schuldentilgung verwendet werden.

11 So auch in Anspielung auf Keynes' berühmtes Zitat („In the long run, we are all dead.") Blinder/Solow: „Since we are all Keynesians now in the short run, those of us, who are not dead in the long run are at least near-monetarists." 
Dem Grunde nach ist dies keine Abkehr von der produktivitätsorientierten Philosophie, daß gegenwärtige Verschuldung nur dann unschädlich ist, wenn dadurch erzielte zukünftige Vorteile per Saldo positiv ausfallen. Es ist vielmehr eine Übertragung der Gedankengänge vom einzelnen schuldfinanzierten (Investitions-)Objekt zur gesamtwirtschaftlichen (Entwicklungs-)Situation. Heute wissen wir, daß dadurch die Messungs- und Zurechnungsprobleme und die Frage nach der Produktivität bzw. Umwegsproduktivität kreditfinanzierter Aktivitäten keineswegs gelöst sind. Der Übergang zur situationsorientierten Verschuldungsregel wurde damals freilich von allen als Fortschritt gerühmt. ${ }^{12}$ Öffentliche Schulden werden nicht nur im Kontext des (vorübergehenden) Deficit Spending, sondern durchaus auch als (dauerhafte) Alternative zur Finanzierung eines höheren Staatsanteils diskutiert. ${ }^{13}$ Von politischer Seite wird dann später konstatiert, daß „(d)ie Auseinandersetzung über Notwendigkeit und Gefahren der Staatsverschuldung... in den letzten Jahren teilweise einem Glaubenskrieg (glich). Dies trifft insbesondere auf die mit einer defizitfinanzierten Fiskalpolitik verbundenen Auswirkungen auf Konjunktur und Beschäftigung zu. “14

Ein weiteres positives Element bezüglich der Erfolgsaussichten gesamtwirtschaftlicher Stabilisierung durch den Bund (und die Länder) ist die im Grundgesetz verankerte Veränderung bei den Steuererträgen für die Gemeinden (Art. 108 Abs. 5) im Zusammenhang mit der Gemeindefinanzreform 1969. ${ }^{15}$ Im Gegenzug zu einer 15\%igen Gewerbesteuerumlage wurde den Gemeinden ab 1970 ein (dem Betrag nach sogar höherer) Anteil an der Lohn- und veranlagten Einkommensteuer zugeteilt. Dies erfolgte nicht nur zur quantitativen Verbesserung der Kommunalfinanzen, sondern hauptsächlich zur Abschwächung der durch die Gewerbesteuer programmierten prozyklischen Ausgabewellen der Gemeinden als Ganzes. Eine damals als gefährlich erkannte konjunkturelle Destabilisierungsursache war damit nachhaltig beseitigt. Schließlich ist im Zusammenhang mit den Änderungen des Grund-

12 Im Überblick vgl. Diekheuer (1978) sowie Hansmeyer (1984), §§ 8-10; siehe auch die 2. Auflage 1970.

13 Vgl. Finanzwissenschaftlicher Beirat beim Bundesministerium für Wirtschaft und Finanzen (1972). So Lambsdorff (1981); dort befindet sich auch ein Überblick zum Stand der Diskussion über öffentliche Verschuldung.

Vgl. im Überblick Elsner/Schüler (1970), insbesondere Erster Teil, B. 


\subsection{Die institutionelle Ausgangssituation}

gesetzes auch die Einführung der Gemeinschaftsaufgaben von Bund und Ländern (im Art. 94a GG) zu nennen. Insbesondere die „Verbesserung der regionalen Wirtschaftsstruktur in Problemgebieten" (z.B. Zonenrand) als ausdrückliches Ziel zeigt die Absicht, nicht nur gesamtwirtschaftlich global zu steuern, sondern den Erfolg auch durch Intervention in problematische regionale Strukturen abzusichern.

Auf dem Gebiet der administrativen Finanzplanung ist im Kontext der Verbesserung der Grundlagen für die volkswirtschaftliche Lenkungsfunktion die Haushalts- und Finanzreform vom August 1969 zu nennen. ${ }^{16}$ Auch hier wird ausdrücklich betont, daß ,(d)er Haushaltsplan... die Grundlage für die Haushalts- und Wirtschaftsführung (ist)“ und daß „(b)ei seiner Aufstellung und Ausführung... den Erfordernissen des gesamtwirtschaftlichen Gleichgewichts Rechnung zu tragen (ist)" ( $\$ 2 \mathrm{HGrG}$ ). Bund und Länder werden verpflichtet, ihr Haushaltsrecht bis zum 1. Januar 1972 nach den Grundsätzen dieser Bundesrahmenordnung zu regeln $(\S 1 \mathrm{HGrH})$; die Bundeshaushaltsordnung wird gleichzeitig mit dem Haushaltsgrundsätzegesetz beschlossen und ist im zitierten $\S 2$ textgleich. Die gebietskörperschaftliche Koordination übernimmt der Finanzplanungsrat (unter Vorsitz des Bundesfinanzministers), und diese Institution ist unter dem Vorsitz des Bundeswirtschaftsministers mitgliedergleich mit dem Konjunkturrat ( $\$ 18$ StabWG). Damit wird die finanzpolitische Kooperation aus föderaler Sicht zumindest den Regeln nach gesichert.

Neben den Vorkehrungen für die Haushalts- und Finanzpolitik ist insbesondere noch auf das Arbeitsförderungsgesetz von Juni 1969 hinzuweisen. Vor dem Hintergrund einer erfolgreichen makroökonomischen Steuerung der Vollbeschäftigung durch das Stabilitäts- und Wachstumsgesetz sollen hier die mikroökonomischen Probleme der Förderung der beruflichen Aus- und Fortbildung sowie der Verbesserung der Beschäftigtenstruktur gelöst werden. Die im wesentlichen reaktive antizyklische Konjunktur- und Beschäftigungspolitik wird damit ergänzt um eine vorausschauende bzw. vorbeugende Arbeitsmarktpolitik, die - ebenfalls dem Vollbeschäftigungsziel ver-

16 Siehe dazu Leicht (1970). „Ziel der Haushaltsreform ist es, Inhalt und Methoden des öffentlichen Haushaltswesens neuen Aufgaben und Erkenntnissen entsprechend neu zu gestalten“ (S. 9). Gemeint ist damit vor allen Dingen „die Anerkennung der wirtschaftspolitischen Budgetfunktion in Bund und Ländern" (S. 10). 
pflichtet - strukturelle Anpassungsprozesse erleichtern und diesbezüglich verursachte Arbeitslosigkeit vermindern soll.

Die institutionellen Rahmenbedingungen sind zusammengesehen konsequent zielgerichtet und systematisch erarbeitet worden. Dabei ist die Mitentscheidung der SPD in der "großen Koalition" und insbesondere die Rolle von Wirtschaftsminister Karl Schiller ein wichtiger Grundpfeiler für die Finanzund Wirtschaftspolitik im Rahmen einer sozial-liberalen Regierungsverantwortung. Es ist vor diesem Hintergrund durchaus verständlich, daß sich Stabilisierungspolitik nicht nur auf ihre eigenständige Aufgabe der Glättung von Konjunkturzyklen beschränkt, sondern auch der Absicherung allokativer Veränderungen zugunsten der Staatswirtschaft den nötigen Rückhalt geben soll. ${ }^{17}$

Mit einer (im heutigen Rückblick: einmalig) günstigen Ausgangskonstellation - nicht nur für die statische Momentaufnahme des Jahres 1969, sondern auch für die dynamische Beobachtung eines zurückliegenden 4-Jahres-Zeitraums - bezüglich der im $\S 1$ StabWG aufgeführten Indikatoren für ein gesamtwirtschaftliches Gleichgewicht im Rücken und mit einer Reihe konzeptionell abgestimmter erfolgverheißender Institutionen im Sinne von kodifizierten, auf ein gemeinsames Ziel ausgerichteten Regelwerken zur Seite, ist es nicht verwunderlich, daß sich die wirtschaftspolitisch Verantwortlichen in der sozial-liberalen Koalition gut ausgerüstet glauben für das, was vor ihnen liegt. Die positiven Erfahrungen sozialdemokratisch beeinflußter Wirtschaftspolitik in der vorangegangenen CDU-SPD-Koalition tragen dazu bei, den Glauben an die Machbarkeit gesamtwirtschaftlicher Lenkung zu stärken. Ein „Programm der inneren Reformen“ aufzulegen ${ }^{18}$ und dies im Verbund mit erfolgreicher Vorsorge für bzw. Wiederherstellung von gesamtwirt-

17 Vgl. Oberhauser (1975). Oberhauser konstatiert zwar unmißverständlich, daß „die antizyklische Finanzpolitik in der Bundesrepublik Deutschland kaum als besonders erfolgreich bezeichnet werden (kann)“ (S. 1), kommt aber immerhin später zu dem Schluß, daß bei einem „,asymmetrischen Einsatz der Mittel“... „Stabilitätspolitik in den Dienst einer Erhöhung des nominellen und realen Staatsanteils" gestellt werden kann. „Eine derartige asymmetrische Einnahmen-Ausgaben-Politik hemmt nicht, sondern erleichtert sogar die Erhöhung der Staatsquote... . Die Voraussetzungen dafür zu schaffen, ist allerdings eine schwierige Aufgabe" (S. 92).

Vgl. Scharpf (1974). 
schaftlichem Gleichgewicht mit Preisniveaustabilität und Vollbeschäftigung in einer „Politik aus einem Guß" (Schiller) bewerkstelligen zu wollen, ist aus damaliger Sicht eigentlich nicht als Utopie zu bezeichnen. Seit Ende der 60er Jahre verbreitet sich allgemein in Theorie und Praxis die Hoffnung auf Steigerung der Effizienz des politischen Planungs- und Entscheidungsprozesses. Im Mittelpunkt steht dabei die Konzeption der politischen Programmplanung mit der Kosten-Nutzen-Analyse als Herzstück ${ }^{19}$. Durch sie soll die input-orientierte Kameralistik überwunden werden zugunsten einer output-orientierten Staatswirtschaft, die auf ökonomische Effizienzkriterien ausgerichtet ist. Angesichts dieser generellen Tendenz ist der Optimismus bezüglich der Steuerbarkeit der Konjunktur im Sinne der volkswirtschaftlichen Lenkungsfunktion nur ein Teil eines umfassenderen Planungsenthusiasmus im Sinne der politischen Programmfunktion des öffentlichen Haushaltes.

19 Siehe Recktenwald (1970) sowie Rürup (1971). 


\subsection{Die Haushalts- und Finanzpolitik vor und nach der Rezession 1975}

Vor dem Hintergrund der einschlägigen Jahreswirtschaftsberichte, Finanzberichte und Regierungserklärungen als direkte Publikationen der Bundesregierung ${ }^{20}$ soll die Entwicklung der Wirtschafts- und Finanzpolitik der sozialliberalen Koalition in ihren wesentlichen Aspekten nachgezeichnet werden. Obwohl bis heute (ungeachtet der jeweiligen politischen Konstellation der Koalition) gilt, da $\beta$ keiner der regierungsamtlich veröffentlichten Berichte eine schonungslose Kritik an der eigenen Politik enthält, sondern geprägt ist von strategischer Rechtfertigung der eigenen Ziele und Konzepte sowie von unvermeidlicher Parteilichkeit ${ }^{21}$, lassen sich doch allgemeine Tendenzen recht gut daraus ableiten. Dies deshalb, weil zum einen wegen des Erhalts der Glaubwürdigkeit dem reinen politischen Strategiedenken Grenzen gesetzt sind und weil zum anderen die idealisierten Projektionen sich jährlich an der tatsächlich eingetretenen Entwicklung messen lassen müssen, also durch die ex post nachweisbaren tagespolitischen Ereignisse eine gewisse Relativierung erfahren. So gesehen stellen die dargelegten Ziele und Konzeptionen eine Art Kompromiß oder Extrakt der bis dahin abgelaufenen Diskussion innerhalb der Bundesregierung und zwischen den einzelnen gesellschaftichen Gruppen dar.

Ausgangspunkt sind der Jahreswirtschaftsbericht 1970 und die Regierungserklärung Willy Brandts vom 28 . Oktober $1969^{22}$, in denen die wirtschaftspolitischen Perspektiven und Zielsetzungen der nächsten Jahre dargelegt werden. So waren zu Beginn der Regierungsperiode für die kommenden Jahre umfangreiche Reformen in allen gesellschaftspolitisch relevanten Bereichen geplant - angefangen bei einer Verwaltungsreform und einer Reform des öffentlichen Dienstrechts über die Schaffung eines Arbeitsgesetzbuches und eines Sozialgesetzbuches bis hin zu einer Reform des Betriebs-

20 Die Jahresgutachten des Sachverständigenrates und die Bundesbankberichte bestätigen die anhand der Jahreswirtschaftsberichte und der Regierungserklärungen beschriebene Entwicklung, werden jedoch nicht gesondert zitiert.

Lediglich die gewählten Formulierungen wechseln von „werden wir erreichen...“ über „wird angestrebt...“ bis zu „konnte nicht verhindert werden...“ (mit impliziter Schuldzuweisung an fremdverursachte Restriktionen).

Vgl. Brandt (1969), S. 1121-1128. 
verfassungsgesetzes und des Personalvertretungsgesetzes. ${ }^{23}$ Dieser Reformeifer wird durch das von Bundeskanzler Brandt als Motto formulierte „Mehr Demokratie wagen" schlagwortartig hervorgehoben. Er galt verständlicherweise nicht den Konzepten für die quantitative Steuerung der gesamtwirtschaftlichen Entwicklung, da die Bundesrepublik seit ihrer Gründung eine Periode beispielloser Prosperität durchlaufen hatte und auch die erste Rezession nach der Rekonstruktionsphase durch ihre rasche Überwindung keinerlei Anlaß zum Pessimismus gab. Politische Reformen waren dagegen auf die gesellschaftlichen Rahmenbedingungen gerichtet, und wann sonst, wenn nicht auf Basis einer prosperierenden Ökonomie, können soziale Reformen mit Aussicht auf Erfolg angegangen werden. Daneben war in der Wirtschaftspolitik geplant, die ,mit dem Stabilitäts- und Wachstumsgesetz und seiner Anwendung sowie der Modernisierung der Strukturpolitik eingeleitete Reform und Modernisierung der Wirtschaftspolitik durch Maßnahmen zur Stärkung der marktwirtschaftlichen Ordnung “24 fortzusetzen. Diese Maßnahmen sahen insbesondere eine Intensivierung des Wettbewerbs, eine Auflockerung administrativer Preisregelungen sowie die Förderung der Vermögensbildung in breiten Schichten der Bevölkerung - vor allem der Arbeitnehmerschaft - vor. ${ }^{25}$

Einen besonderen Stellenwert innerhalb der wirtschaftspolitischen Konzeption nahm die „Konzertierte Aktion“ der Gebietskörperschaften, der Gewerkschaften und der Unternehmerverbände ein. Für eine wirksame Koordinierung der einzelwirtschaftlichen Pläne war nach Ansicht der Bundesregierung entscheidend, „daß alle Beteiligten aufgrund ihrer Einsicht in die gesamtwirtschaftlichen Zusammenhänge und in die Rückwirkungen ihres eigenen Verhaltens auf das Verhalten der übrigen ihre gemeinsame Verantwortung erkennen und ihre autonomen Entscheidungen entsprechend treffen ${ }^{\text {“26 }}$. Hierzu stellte die Bundesregierung mit ihrer Jahresprojektion und der Erläuterung der geplanten Wirtschafts- und Finanzpolitik Orientierungsdaten zur Verfügung, an denen die gesellschaftlichen Entscheidungsträger ihr Handeln ausrichten konnten - und sollten. Gleichzeitig sollte die konzertierte Aktion jedoch Spielraum für verteilungspolitische Korrekturen behalten. Insgesamt

\footnotetext{
23 Vgl. ebenda, S. 1124 und 1126.

24 Jahreswirtschaftsbericht 1970 der Bundesregierung, S. 22.

25 Vgl. ebenda, S. 22-24.

26 Ebenda, S. 7.
} 
waren zur Erreichung der mit dem Stabilitäts- und Wachstumsgesetz angestrebten wirtschaftspolitischen Ziele - Stabilität des Preisniveaus, hoher Beschäftigungsstand, außenwirtschaftliches Gleichgewicht und stetiges und angemessenes Wirtschaftswachstum - laut Sachverständigenrat und Regierung ein hohes $\mathrm{Ma} B$ an

- Prognosefähigkeit der Experten,

- Vertrauen der Politiker in die Prognosen,

- Handlungsbereitschaft sowie

- Koordinierung und Konzertierung der autonomen wirtschaftlichen Kräfte notwendig. Eine erfolgreiche Konjunktursteuerung konnte ihrer Ansicht nach umso eher erreicht werden, je größer die Einsicht vor allem der wirtschaftlichen Entscheidungsträger in die konjunkturellen Zusammenhänge und ökonomischen Wirkungsmechnismen war. Zur weiteren Verbesserung der Konjunkturdiagnose und -prognose wurde daher eine Verbesserung des statistischen Informationssystems angestrebt ${ }^{27}$ Prinzipiell schien die Bundesregierung jedoch keinen Zweifel daran zu hegen, daß die Ziele des Stabilitätsund Wachstumsgesetzes bei entsprechender Kenntnis und Kooperationsbereitschaft der am Wirtschaftsprozeß Beteiligten erreichbar waren. Dieser Optimismus bezüglich der „Machbarkeit“ stabilitäts- und wachstumsgerechter Wirtschaftspolitik bringt sie nicht zuletzt zum Ausdruck, indem sie dem Stabilitätsgesetz ,bedeutende Fortschritte hinsichtlich einer laufenden Orientierung der Exekutive an der konjunkturellen Entwicklung ${ }^{\text {c28 }}$ und eine Vergrößerung der Anpassungsmöglichkeiten an konjunkturpolitische Erfordernisse attestiert und der Sachverständigenrat ihrer Meinung nach die „rechtlichen Möglichkeiten prophylaktischen Handelns ${ }^{\text {‘29 }}$ eher unterschätzt.

Der Planungs- und Lenkungsoptimismus ging jedoch noch weiter. Zum einen sollten die Orientierungsdaten der Bundesregierung - und damit die geplante Wirtschafts- und Finanzpolitik - dem „Grundsatz der sozialen Symmetrie ${ }^{630}$ Rechnung tragen. Auf längere Sicht sollten die Arbeitnehmer stärker am Volkseinkommen beteiligt werden. Darüber hinaus sollte aber auch eine wachstumsorientierte Strukturpolitik betrieben werden, die die Be-

Vgl. ebenda, S. 7.

Ebenda, S. 8.

Ebenda, S. 8.

Ebenda, S. 21. 
reiche Regional-, Sektoral- und Unternehmensgrößenpolitik umfaßt. Durch eine „Strukturpolitik aus einem Guß“ sollten so verschiedene Bereiche wie die Raumordnungspolitik, die Agrarstrukturpolitik, die Arbeitsmarktpolitik, die Berufsbildungspolitik, die regionale und sektorale Wirtschaftspolitik, die Politik für kleine und mittlere Unternehmen, die Städtebaupolitik, die Technologiepolitik und die Verkehrspolitik aufeinander abgestimmt und stärker miteinander verknüpft werden. So sollte beispielsweise die (Berufs-) Bildungspolitik mit einer zukunftsorientierten Aus- und Fortbildung zu einer schnellen Anpassung der Arbeitnehmer an die fortschrittsbedingten wirtschaftlichen Veränderungen beitragen. ${ }^{31}$ Im Rückblick erscheint es zunächst schon verwunderlich, wie man glauben konnte, all diese Bereiche unter einen gemeinsamen - noch dazu konjunktur- und wachstumsgerechten - Hut zu bekommen. Daraus der damaligen Gegenwart aus heutiger Sicht einen Vorwurf zu machen, sollte allerdings nicht vorschnell erfolgen. Gerade die bereits erwähnte beispiellose Prosperität über ein Jahrzehnt und überhaupt das weltweit gelobte „deutsche Wirtschaftswunder" der Nachkriegszeit sind Anlaß genug, mehr für erreichbar zu halten als auf Dauer erreicht werden konnte - zumal am Beginn einer neuen Regierungskonstellation, wo notwendigerweise alle Politikfelder neu zu besetzen sind.

Noch deutlicher wird die Zielsetzung der Bundesregierung im Jahreswirtschaftsbericht 1971. Hier heißt es: „Die Bundesregierung wird ihre Politik zur Stärkung der marktwirtschaftlichen Ordnung und eines freien Außenhandels intensivieren. Wettbewerbspolitik, Konjunkturpolitik, Strukturpolitik und Gesellschaftspolitik sollen zu einem Gesamtkonzept verschmolzen wer-

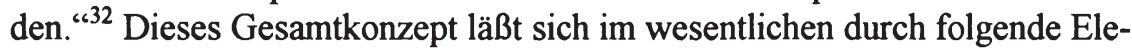
mente charakterisieren:

- eine vorausschauende Konjunkturpolitik in Form einer Globalsteuerung,

- die Sicherung des sozialen Ausgleichs und des sozialen Fortschritts, u.a. durch eine gerechtere Vermögensverteilung,

- die Erhaltung der Funktionsfähigkeit der dezentralen Selbststeuerungskräfte des Marktes sowie

- eine Strukturpolitik in Form einer angebotserweiternden Wachstumspolitik, die Anreize für eine wachstumsfördernde intersektorale und räumliche

31 Vgl. ebenda, S. 24-29 sowie Brandt (1969), S. 1125 f.

32 Jahreswirtschaftsbericht 1971 der Bundesregierung, S. 29. 
Allokation von Kapital und Arbeit schafft und den Strukturwandel begünstigt. $^{33}$

Letztere beinhaltete eine Förderung von Forschung und Entwicklung sowie eine berufliche Bildung und Fortbildung, die dazu beitragen sollte, „Unternehmer und Arbeitnehmer besser über die volkswirtschaftlichen, betriebswirtschaftlichen und technischen Zusammenhänge ${ }^{\text {“34 }} \mathrm{zu}$ informieren. Allgemein lag dem Wirtschaftskonzept der sozial-liberalen Koalition zu Beginn ein relativ mechanistisches Verständnis des Wirtschaftsablaufs und seiner Steuerbarkeit zugrunde. Es war sogar von einem „fine tuning“ die Rede.

Die Finanzpolitik der kommenden Jahre sollte dabei durch Solidität gekennzeichnet sein. Als wichtige Vorhaben wurden eine Steuerreform zur Schaffung eines gerechten, einfachen und überschaubaren Steuersystems sowie eine Reformierung der Abgabenordnung genannt. Ebenso sollte die bereits eingeleitete Finanzreform vollendet werden. Besonders hervorgehoben wurde dabei die Zusammenarbeit von Bund, Ländern und Gemeinden im Finanzplanungsrat. Die Finanzsituation selber sieht Brandt als „weniger günstig“ an „als sie von bestimmter Seite dargestellt wurde“335. Dies erstaunt (nicht nur) im Rückblick, da - wie noch gezeigt werden wird - die Haushaltslage der Gebietskörperschaften besser war als in der gesamten zurückliegenden Periode ${ }^{36}$ und so gut wie sie in den darauffolgenden Jahren bis heute nie wieder gewesen ist.

Wie sich schnell zeigte, wurde die Möglichkeit einer Koordinierung der Interessen bzw. die Einsichtsfähigkeit der Handelnden in gruppenspezifisch jeweils nachteilige Beiträge zu den „Erfordernissen eines gesamtwirtschaftlichen Gleichgewichts“ insbesondere im Rahmen des Verteilungskampfes überschätzt. ${ }^{37}$ Die Äußerungen zur geplanten Wirtschaftspolitik wurden dementsprechend zunehmend vorsichtiger und verhaltener. In der Ausein-

Vgl. ebenda, S. 29-36.

Ebenda, S. 34.

Brandt (1969), S. 1123.

Lediglich die Länder erzielen Anfang der 60er Jahre bessere Tragfähigkeitsindikatoren.

$37 \mathrm{Zu}$ einer Kritik an der Stabilisierungspolitik vgl. etwa Beyfuss (1977) oder Kloten (1976). Zur kritischen Analyse des Stabilitätsgesetzes selbst vgl. auch Hansmeyer (1973a, 1973b, 1973c, 1977) oder Rürup/Siedenberg (1974). 
andersetzung mit dem Sachverständigenratsgutachten 1972 im Jahreswirtschaftsbericht 1973 begrüßte die sozial-liberale Regierung dessen ,versachlichende Wirkung", die dazu beitrage, die Grenzen wirtschaftspolitischer Steuerungsmöglichkeiten zu verdeutlichen und „überhöhte Ansprüche“ und Erwartungen an die Politik zu vermeiden. Denn: „In Überschätzung des institutionell und situationsgerecht „Machbaren“" werden nämlich sonst von der Bundesregierung oft Maßnahmen erwartet, über die sie aufgrund zweifelsfreier, zeitgerechter Indikatoren nur mutig zu entscheiden brauche, um der konjunkturellen Entwicklung in all ihren Ausprägungen die gewünschte Richtung zu geben. ${ }^{‘ 38}$ Die Bundesregierung wies außerdem darauf hin, daß im Rahmen der marktwirtschaftlichen Ordnung eine staatliche Beeinflussung des Wirtschaftsprozesses nur indirekt über eine Veränderung der globalen Rahmenbedingungen erfolgen könne. ${ }^{39}$ Als Gründe für die eingeschränkte Wirksamkeit staatlicher Stabilisierungsmaßnahmen wurden u.a. die zunehmende internationale Verflechtung der Volkswirtschaften und die Erwartungen (im Jahreswirtschaftsbericht 1973 konkret die Preis- und Einkommenserwartungen) der Marktteilnehmer genannt ${ }^{40}$ sowie allgemein die begrenzte Vorhersagbarkeit wirtschaftlicher Entwicklung und ordnungspolitischer Grenzen. ${ }^{41}$ Zusätzlich dürfte auch die Tatsache, daß sich die Verteilungskonflikte nur begrenzt im Rahmen der Konzertierten Aktion lösen ließen, zu der insgesamt inzwischen eher skeptischen Einschätzung der Steuerbarkeit des Wirtschaftsablaufs beigetragen haben. Bereits in dieser Anfangsphase wurde zudem deutlich, daß eine restriktive Konjunkturpolitik über die Ausgabenseite des Haushalts bei anhaltender Inflation und einem aufgestauten Bedarf an öffentlichen Leistungen schwierig ist. ${ }^{42}$ War anfänglich von einer Unterschätzung des Stabilitäts- und Wachstumsgesetzes und seiner Instrumentarien die Rede, so hielt die Bundesregierung zu der Zeit bereits eine Überprüfung und Verbesserung des konjunkturpolitischen Instrumentariums - einschließlich des kreditpolitischen Instrumentariums der Deutschen Bundesbank - für nötig. Im Hintergrund spielten dabei die politischen Erfahrungen mit dem maroden System fester Wechselkurse (Bretton Woods) und der dann 1973 erfolgende Übergang zu flexiblen Wechselkursen eine nicht un-

Vil. ebenda, S. 6.

41 Vgl. ebenda, S. 18.

42 Vgl. ebenda, S. 20.
} 
bedeutende Rolle. Die folgenden Jahreswirtschaftsberichte bestätigen und bekräftigen das. Grundsätzlich hielt die sozial-liberale Koalition jedoch zu dieser Zeit noch an der Konzeption des Stabilitäts- und Wachstumsgesetzes fest. $^{43}$

Im Laufe der folgenden Jahre vollzog sich jedoch eine sukzessive Umorientierung von der reinen Nachfragesteuerung in Richtung einer Angebotssteuerung. Eingeleitet wurde diese Abkehr von der Globalsteuerung durch die Ölkrise (Ende 1973) und die ihr folgende Rezession 1975, die die bis dahin schwerste Rezession der Nachkriegszeit darstellte und in der Bundesrepublik die Arbeitslosenraten von 1,2\% auf 4,6\% anschwellen ließen. Erstmals seit 1967 war damit auch das Ziel eines hohen Beschäftigungsstandes gefährdet. Dieser Anstieg der Arbeitslosigkeit wurde in erster Linie auf strukturelle Veränderungen in der Wirtschaft zurückgeführt, die durch den Abschwung lediglich zutage gefördert wurden. Der untere Wendepunkt eines konjunkturellen, mittelfristigen (Juglar-)Zyklus traf zusammen mit dem Beginn eines strukturellen, langfristigen (Kondratieff-)Zyklus, eingeleitet durch die mikroelektronische technische Revolutionierung und den Niedergang sogenannter altindustrieller Strukturen. Eine rasche Überwindung der Rezession wie 1967 trat deshalb nun nicht ein.

Die Umorientierung läßt sich an verschiedenen Punkten festmachen, die eng miteinander verbunden sind:

- Höherer Stellenwert der Strukturpolitik. Aufgrund der Akzentuierung des Strukturwandels durch die Rezession gewann die Strukturpolitik an Gewicht. Durch eine „stärker mittelfristig orientierte Globalsteuerung“ in Form von Hilfe zur Selbsthilfe und durch die Gestaltung staatlicher Rahmenbedingungen sollte der Strukturwandel gefördert werden und dabei auftretende regionale und sektorale Friktionen und soziale Härten sollten abgefedert werden. Im wesentlichen wurden unter Strukturpolitik technologie- und mobilitätsfördernde Maßnahmen verstanden, d.h. Maßnahmen, die zu einer effizienteren Allokation von Kapital und Arbeit führen sollen. ${ }^{44}$ Dabei war keine branchenbezogene, sondern eine gesamtwirtschaft-

43 Vgl. ebenda, S. 9.

44 Vgl. Jahreswirtschaftsbericht 1976 der Bundesregierung, S. 17. 
lich orientierte Wachstumspolitik beabsichtigt. ${ }^{45} \mathrm{Daß}$ einzelne Maßnahmen möglicherweise dennoch eher zu einer Konservierung überholter Strukturen beigetragen haben, widerspricht nicht dieser Absicht.

- Verstärkte Förderung privater Investitionen. Unter wachstumspolitischen Gesichtspunkten wurde den Investitionen eine entscheidende Bedeutung zugesprochen. Private - und komplementär öffentliche - Investitionen wurden als Fundament für das künftige Wachstum betrachtet. Die schwache Investitionsneigung der vorhergehenden Jahre hatte zu einem verminderten Wachstum des Produktionspotentials geführt. Um das Wachstum des Produktionspotentials - und damit das Wachstum des Sozialprodukts und die Beschäftigung - zu fördern, sollte die Investitionsneigung angeregt werden. ${ }^{46}$ Dabei ging die Bundesregierung davon aus, daß bei ausreichender Investitionstätigkeit mehr neue Arbeitsplätze geschaffen als wegrationalisiert werden. ${ }^{47}$ Eine Investitionslenkung in Form von Investitionsge- und -verboten, wie sie von Teilen der SPD gefordert wurde, lehnte die Bundesregierung ab; sowohl aus Effizienzgründen - eine Lenkung würde die Elastizität und Anpassungsfähigkeit der Wirtschaft beeinträchtigen als auch zur Erhaltung der Autonomie der Marktteilnehmer. ${ }^{48}$

- Verstetigung der Finanzpolitik. Bereits im Jahreswirtschaftsbericht 1975 wurde - angestoßen durch den Sachverständigenrat - über eine Änderung der stabilitätspolitischen Konzeption nachgedachtg. Der Sachverständigenrat hatte vorgeschlagen, der Geldpolitik die dominierende Rolle bei der Inflationsbekämpfung zuzuweisen. Die Geldpolitik sollte dabei potentialorientiert sein, d.h. die Geldbasis sollte so gesteuert werden, „daß ein monetärer Spielraum für einen gesamtwirtschaftlichen Ausgabenzuwachs erreicht wird, der dem Wachstum des Produktionspotentials zuzüglich der als unvermeidlich anzusehenden Inflationsrate entspricht. ${ }^{\text {(49 }}$ Die Finanzpolitik sollte hingegen von konjunktur- und stabilitätspolitischen Aufgaben entlastet und verstetigt werden, nicht zuletzt um den Zielkonflikt der Finanzpolitik zwischen der Versorgungsfunktion, der sozialen und der konjunkturpolitischen Funktion zu mildern. Die Bundesregierung teilte die Ansicht des Rates, daß eine Stabilisierungspolitik nicht über einen länge-

Vgl. ebenda, S. 17 sowie Jahreswirtschaftsbericht 1975 der Bundesregierung, S. 14.

Vgl. Jahreswirtschaftsbericht 1975 der Bundesregierung, S. 24 f.

Vgl. Jahreswirtschaftsbericht 1977 der Bundesregierung, S. 5.

Vgl. Jahreswirtschaftsbericht 1976 der Bundesregierung, S. 17 und 31.

So zitiert im Jahreswirtschaftsbericht 1975 der Bundesregierung, S. 25.
} 
ren Zeitraum einseitig zu Lasten der öffentlichen Leistungen gehen könnte. Ebenso hielt der Sachverständigenrat die Flexibilität der Haushalte für eine restriktive Finanzpolitik für nicht ausreichend. Darüber hinaus erwies sich selbst die Flexibilität der Ausgaben innerhalb eines gegebenen Budgets, d.h. ohne eine Beschränkung der Ausgaben, als begrenzt. Dafür sind rechtliche, politisch-soziologische und technisch-ökonomische Bindungen verantwortlich. So hatte eine Arbeitsgruppe des Finanzplanungsrates allein den Anteil der rechtlich fixierten Ausgaben des Bundes 1973 auf 95,5\% - bzw. bei Herausnahme strittiger Positionen auf 79,9\% - geschätzt. Ewringmann kommt bei einer ähnlichen Analyse auf Werte von 93,17-95,86 für die Jahre 1971 bis 1974 bzw., unter Absetzung umstrittener Ansätze, auf Werte von 89,91 und 86,23\% für die Jahre 1973 und 1974, wobei allerdings die Vorausfixierung durch gesetzliche und vertragliche Verpflichtungen bei den einzelnen Ressorts recht unterschiedlich ausfiel. Zwar handelt es sich bei diesen Bindungen um keine ein für allemal festgelegten Restriktionen. Der kurzfristige Handlungsspielraum, auf den es in der Stabilisierungspolitik ankommt, ist jedoch eingeschränkt. Zudem wachsen mit zunehmender europäischer Integration die Bindungen durch die supranationale Ebene der EG. ${ }^{50}$ Dennoch lehnte die Bundesregierung eine Verstetigung der Ausgaben und einen völligen Verzicht auf antizyklische Variationen der Haushalte $a b .^{51}$ In den folgenden Jahren wurden jedoch immer stärker die mittel- und langfristigen Aspekte der Wirtschaftspolitik hervorgehoben. Wesentlich für diesen Wandel in der wirtschaftspolitischen Ausrichtung waren die bereits genannten Strukturprobleme sowie der gewachsene Wachstumspessimismus, der sich vor allem in einer geringen Investitionstätigkeit niederschlug. Nicht zuletzt haben aber wohl auch die eher ernüchternden Erfahrungen mit der keynesianischen Konjunktur- und Wachstumspolitik zu dieser Umorientierung beigetragen. Im Jahreswirtschaftsbericht 1975 wurde zwar noch auf die (kurzfristige) pychologische Wirkung der Haushalte als Impulsgeber verwiesen, später argumentiert die Bundesregierung jedoch mit der Notwendigkeit der Stabilisierung der Erwartungen des Unternehmenssektors zur Belebung der Investitionen. ${ }^{52}$ Auch weiterhin wurde zwar die Notwendig-

50 Vgl. Ewringmann (1975), insbesondere S. 53-93.

Vgl. ebenda, S. $25 \mathrm{f}$.

Vgl. ebenda, S. 26; Jahreswirtschaftsbericht 1976 der Bundesregierung, S. 29; Jahreswirtschaftsbericht 1977 der Bundesregierung, S. 20. 
keit finanzpolitischer Flexibilität hervorgehoben, gleichzeitig wurde jedoch stets auf die Grenzen des konjunkturpolitisch Machbaren hingewiesen. Hatte die Bundesregierung 1975 noch eine Verstetigung der Finanzpolitik abgelehnt, so wurde diese zwei Jahre später explizit als Ziel genannt. ${ }^{53}$ Im Zusammenhang mit der Verstetigung der Finanzpolitik wurde auch und im besonderen die Verstetigung bei den öffentlichen Investitionen (wenn auch nicht so sehr von der Bundesregierung selbst) diskutiert. Der qualitative Hintergrund dieser Diskussion war die Forderung nach einem stärkeren Gewicht der allokativen Versorgungsfunktion der Finanzpolitik gegenüber der (antizyklischen) Lenkungsfunktion. Es wurde abgelehnt, die aus langfristig begründbaren Allokationsinteressen erforderlichen öffentlichen Investitionen faktisch kurzfristig nach Maßgabe von konjunkturellen Lenkungsinteressen schwanken zu lassen.

- Verlagerung der finanzpolitischen Maßnahmen von der Ausgaben-auf die Einnahmenseite des Budgets. Bei anhaltender Inflation ist eine Begrenzung der Ausgaben stets mit Einschnitten in die öffentliche Leistungserbringung verbunden. Die Bereitstellung öffentlicher Güter und Dienstleistungen kann jedoch nicht beliebig eingeschränkt werden. Daher - und nicht zuletzt wohl auch wegen der im vorhergehenden Punkt genannten mangelnden Flexibilität öffentlicher Ausgaben - erfolgte die Stabilisierungspolitik bereits vor 1975 vermehrt über die Einnahmenseite des Budgets. ${ }^{54}$ Außerdem impliziert die verstärkte Angebotsorientierung eine solche Verschiebung. Das wesentliche Instrument der Angebotspolitik ist die Schaffung eines allokationsorientierten Steuersystems. Mit Hilfe der Steuerpolitik - in Form von Entlastungen bei der Einkommensteuer, Körperschaftsteuer, Gewerbesteuer und Vermögensteuer - sollen wachstumsund beschäftigungsfördernde Anreize für die private Wirtschafts-, insbesondere die private Investitionstätigkeit gesetzt werden. ${ }^{55}$

- Verbesserung der Haushaltsstruktur. Auf der Einnahmenseite war hiermit die Umgestaltung des Steuersystems von investitionshemmenden zu investitionsfreundlichen Steuern gemeint. Hierzu gehörten Maßnahmen wie erhöhte Abschreibungsmöglichkeiten oder allgemein Entlastungen bei den Unternehmensteuern. Daneben gab es aber auch - sozialpolitisch moti-

53 Vgl. Jahreswirtschaftsbericht 1977 der Bundesregierung, S. 20.

54 Vgl. Jahreswirtschaftsbericht 1975 der Bundesregierung, S. 26.

$55 \mathrm{Vgl}$. bspw. Jahreswirtschaftsbericht 1978 der Bundesregierung, S. $21 \mathrm{f}$. 
vierte - Entlastungen bei der Einkommensteuer. Die Verbrauchsteuern vor allem die Mehrwertsteuer - wurden hingegen erhöht. ${ }^{56}$ Auf der Ausgabenseite wurde eine Umstrukturierung hin $\mathrm{zu}$ mehr investiven und weniger konsumtiven Ausgaben angestrebt. Zur Unterstützung der privaten Investitionstätigkeit wurde ein "Programm für Zukunftsinvestitionen“ (ZIP) aufgelegt, das einen Ausbau der öffentlichen Infrastruktur vorsah. Dem ZIP wurden jedoch enge Grenzen gesetzt, indem solche Projekte ausgewählt werden sollten, die mit möglichst geringen Folgekosten verbunden waren. ${ }^{57}$ Denn allgemein sollte der Ausgabenanstieg begrenzt werden. Der Staatsverbrauch sollte zurückgeführt und das strukturelle Defizit abgebaut werden. Am 10. September 1975 beschloß die Bundesregierung ein Haushaltsstrukturgesetz, das am 1. Januar 1976 in Kraft trat und eine Reihe von Maßnahmen zur Verbesserung der Haushaltsstruktur vorsah. $^{58}$ (Dazu gehörten beispielsweise Einsparungen im öffentlichen Dienst und Änderungen des Arbeitsförderungsgesetzes - u.a. Erhöhung des Beitragssatzes zur Arbeitslosenversicherung, Einschränkungen im Bereich der beruflichen Ausbildung, Fortbildung und Umschulung). ${ }^{59}$

- Zuweisung der Verantwortung für die Beschäftigung zurück an den privaten Sektor. Nach dem Stabilitäts- und Wachstumsgesetz liegt die Verantwortung für den Beschäftigungsstand (wie auch für die anderen wirtschaftspolitischen Zielsetzungen) sowohl beim Staat als auch bei den Tarifvertragsparteien. Im Rahmen der Konzertierten Aktion sollen die Interessen und hierüber das Handeln der am Wirtschaftsprozeß beteiligten Gruppen aufeinander abgestimmt werden, indem beispielsweise LohnLeitlinien für die Tarifverhandlungen festgelegt wurden. Faktisch konnte aber über das Instrument der Konzertierten Aktion kein Konsens der beteiligten Gruppen hergestellt werden. (Taktisch ist sie dagegen oftmals dazu benutzt worden, die Verantwortung für die Verletzung der wirtschaftspolitischen Ziele auf die jeweils anderen Gruppen zu überwälzen; daran ist sie letztlich gescheitert.) In den Jahren nach 1975 sind mehrere

56 Vgl. hierzu jeweils den Abschnitt „Steuerpolitik“ in den Jahreswirtschaftsberichten der Bundesregierung sowie die Chroniken zur Wirtschafts- und Finanzpolitik in den Jahresgutachten des Sachverständigenrates zur Begutachtung der gesamtwirtschaftlichen Entwicklung und den Geschäftsberichten der Deutschen Bundesbank. Vgl. Jahreswirtschaftsbericht 1977 der Bundesregierung, S. 9.

Vgl. Jahreswirtschaftsbericht 1976 der Bundesregierung, S. 26 f.

Zu den Änderungen in der Haushaltsstruktur vgl. auch Abschnitt 1.4. 
arbeitsmarktpolitische Programme aufgelegt worden. Diese Programme beinhalteten jedoch keine Beschäftigungspolitik im keynsianischen Sinne, sondern Maßnahmen zur Mobilitätsforderung, höhere Mittel für Arbeitsbeschaffungsmaßnahmen und Arbeitsvermittler, Investitionsanreize und Lohnkostenzuschüsse, d.h. vor allem Maßnahmen, die auf die Allokation gerichtet waren. Es wurde allerdings zunehmend deutlicher, daß die bestehende Arbeitslosigkeit nicht nur ein mittel-, sondern ein langfristiges Problem darstellte. Das auch früher immer wieder auftauchende Lohnkostenargument wurde nun von der Bundesregierung verstärkt herangezogen. Letztlich steht hinter dieser Entwicklung jedoch eine gewisse Resignation vor dem Beschäftigungsproblem, die in der verstärkten Zuweisung der arbeitsmarktpolitischen Verantwortung zurück an den privaten Sektor mündet. ${ }^{60}$

Insgesamt werden also mittel- und langfristige Aspekte der Wirtschaftspolitik stärker betont. Mit Maßnahmen wie der Intensivierung der Investitionsförderung sowie der Verlagerung der Finanzpolitik auf einnnahmenseitige Maßnahmen - und hier insbesondere die Umstrukturierung hin zu einem investitionsfreundlicheren Steuersystem - kommen zunehmend angebotsorientierte Elemente in die Wirtschaftspolitik. Noch verbleibende Elemente einer Nachfragepolitik entfernen sich in wachsendem Maße von den ursprünglich im Stabilitäts- und Wachstumsgesetz vorgesehenen Maßnahmen, so daß das Gesetz - wie sich im nachhinein bestätigt - praktisch „vergessen“, d.h. als nicht mehr vollzugsverbindlich angesehen wurde. ${ }^{61}$

Eng verknüpft mit dem Wandel in der wirtschaftspolitischen Konzeption ist das Bestreben, den Haushalt selbst zu konsolidieren. Erstmals war hiervon im Jahreswirtschaftsbericht 1973 die Rede. Einer auch mittelfristig für notwendig erachteten Steigerung der öffentlichen Gesamtausgaben sollten entsprechende Einnahmenverbesserungen gegenüberstehen. Gleichzeitig wurde eine Schuldendeckelverordnung nach $\S \S 19$ ff. des Stabilitäts- und Wachstumsgesetzes erlassen, durch die die Ausgabenmöglichkeiten der Gebietskörperschaften begrenzt werden sollten. Hierbei ging es jedoch um eine

60 Vgl. hierzu jeweils den Abschnitt „Preis- und einkommenspolitisches Verhalten“ in den Jahreswirtschaftsberichten der Bundesregierung.

61

Vgl. Hansmeyer (1977). 
(kurzfristige) Begrenzung der gesamtwirtschaftlichen Nachfrage zur Stabilisierung des Preisniveaus. ${ }^{62}$ Mittelfristig waren weitere Ausgabensteigerungen vorgesehen.

Die Konsolidierungsbemühungen ab 1976 erhielten einen anderen Akzent. Vor allem in der Anfangsphase der sozial-liberalen Regierung hatte eine überdurchschnittliche Ausweitung des Ausgabenvolumens stattgefunden, dem die Einnahmenentwicklung nicht standhielt, so daß der Haushalt des Bundes trotz eines zufriedenstellenden Sozialproduktswachstums einen negativen Finanzierungssaldo aufwies. Von 1970 bis 1974 wuchs die Verschuldung des Bundes um 45,3\%. Seine Schuldenquote (hier definiert als Schuldenstand am Ende eines Jahres in Relation zum Bruttosozialprodukt) entsprach jedoch 1974 immer noch dem Stand von 1970, weil dem Wachstum der Bundesverschuldung ein ähnliches Wachstum des Bruttosozialproduktes gegenüberstand. Der eigentlich problematische Anstieg des Schuldenstandes und der Schuldenquote sowie der insgesamte stärkste Anstieg der Bundesschuld mit 54,3\% erfolgte im Rezessionsjahr 1975 und in den darauffolgenden Jahren. ${ }^{63}$ Um ein weiteres Wachstum der Verschuldung zu verhindern, sah sich die Bundesregierung mittelfristig zu einer Konsolidierung gezwungen. Ein weiteres Wachstum der Ausgaben sollte verhindert und das kontinuierlich gewachsene strukturelle Defizit durch eine generelle Verbesserung der Haushaltsstruktur abgebaut werden. Durch die Konsolidierung sollte außerdem der Kapitalmarkt entlastet werden, um die Fremdfinanzierungsmöglichkeiten des privaten Sektors zu verbessern und damit einen erweiterten Spielraum für private Investitionstätigkeit zu schaffen (Abbau von Crowding Out).

Den Abschluß dieser Bemühungen bildet die sog. „Operation ' 82 “, deren wesentliche Bestimmungen am 1. Januar 1982 in Kraft traten. Dieses Programm sah umfassende und vielfältige Maßnahmen zur Förderung von Wachstum und Beschäftigung sowie zur Verbesserung der Haushaltssituation vor. So waren Ausgabenkürzungen im Rahmen des Gesetzes zur Konsolidierung der Arbeitsförderung, eine Anhebung der Sätze für Tabaksteuer, Branntweinabgaben und Sektsteuer, eine Anhebung des Beitragssatzes der

62 Vgl. auch Hansmeyer (1977), S. 611.

63 Vgl. ausführlicher Abschnitt 1.3. 
Bundesanstalt für Arbeit, Abschreibungserleichterungen für Gebäude und bewegliche Wirtschaftsgüter, Kürzungen beim Kindergeld sowie Wegfall von Steuervergünstigungen vorgesehen.

Die Regierungszeit der sozial-liberalen Koalition läßt sich zusammenfassend gemäß der wirtschaftspolitischen Konzeption in zwei Phasen einteilen, wobei die Rezession 1975 einen Wendepunkt markiert. In der ersten Phase von 1969 bis 1975 überwiegt eine nachfrageorientierte Politik, während sich in den Jahren 1976 bis 1982 verstärkt eine Angebotsorientierung durchsetzt. Dabei kommt es freilich nicht zu einer vollständigen Ablösung der einen durch die andere Konzeption; vielmehr bleibt gezielt ein Mix von Nachfrage- und Angebotspolitik bestehen, weil nicht alle Arbeitslosigkeit als strukturell, sondern ein Teil davon nach wie vor als konjunkturell eingeschätzt wird.

Eine eindeutige Erschwerung exakter Diagnose bedeuten die Veränderungen der internationalen Rahmenbedingungen und damit der Determinanten eines außenwirtschaftlichen Gleichgewichtes. Die relativen Preise und Mengen werden durch die Ölpreisschocks und die Neuordnung der Wechselkursbeziehungen nachhaltig verändert. In dieser Hinsicht wird die Haushalts- und Finanzpolitik der sozial-liberalen Koalition den sich ändernden Rahmenbedingungen durchaus gerecht. $\mathrm{Da} \beta$ ein eindeutiges Muster nicht erkennbar ist, liegt auch daran, daß zu der damaligen Zeit die realwirtschaftlichen Veränderungen so eindeutig nicht zutage traten. Auf jeden Fall kann konstatiert werden, daß der Wandel der konjunktur- und wachstumspolitischen Ausrichtung sich nicht erst mit der „Wende“ zur christlich-liberalen Koalition im Oktober 1982 vollzieht, sondern bereits Mitte der 70er Jahre beginnt. Ein wesentlicher Unterschied in der Politik vor und nach dem Regierungswechsel liegt jedoch darin, daß die sozial-liberale Koalition auch weiterhin Maßnahmen zur Anregung der Nachfrage getroffen und gezielt ein Nebeneinander von Nachfrage- und Angebotspolitik zugelassen hat ${ }^{64}$, während die konservativ-liberale Koalition auf eine reine Angebotspolitik umstellte. ${ }^{65} \mathrm{Im}$ internationalen Verein mit der britischen und amerikanischen Wirtschaftspo-

64 Vgl. Jahreswirtschaftsbericht 1979 der Bundesregierung, S. 24.

65 Nicht zuletzt die Flügelkämpfe innerhalb der SPD haben verhindert, daß sich eine stärker angebotsorientierte und damit Allokationspolitik gegenüber einer Verteilungspolitik durchsetzen ließ. 
litik (nach ihren Protagonisten als „Thatcherism“ bzw. „Reaganomics“ genannt) ${ }^{66}$ war dies auch leichter politisch durchzusetzen. So jedenfalls könnte verständlich werden, daß die seit 1981 und dann wieder seit 1983 erheblich ansteigende Massenarbeitslosigkeit und ihre Verfestigung mit Zunahme der Langzeitarbeitslosigkeit zwar vermutlich mit ein Grund für den 1982er Regierungswechsel war, aber offenbar die Dauerhaftigkeit der christlich-liberalen Koalition seit 1982 nicht beeinträchtigen konnte. Anders ausgedrückt: Mit dem (weltweit) proklamierten Primat der Allokation und der Zurückweisung der politisch-instrumentellen Verantwortung für einen hohen Beschäftigungsstand (unter Hinweis auf die dafür ursächlich haftenden und langfristig wirkenden Angebotsbedingungen) ist die christlich-liberale Koalition bis heute länger und für ihr politisches Überleben offenbar weniger gefährdend umgegangen als ihre sozial-liberale Vorgängerregierung.

66 Siehe Barker (1985) und Niskanen (1988), insbesondere 2. und 3. Kapitel. 


\subsection{Auswirkungen der Finanzpolitik auf die Defizit- und Schuldenentwicklung}

Wie einleitend angedeutet wurde, haben nicht erst die hohen Kosten der Deutschen Einheit und der Vertrag von Maastricht die Aufmerksamkeit der deutschen Öffentlichkeit auf die Entwicklung des staatlichen Haushalts und der Verschuldung gelenkt. Bereits in den siebziger Jahren sah sich die Bundesrepublik - wie die meisten Industrie- und Entwicklungsländer - einer wachsenden Staatsverschuldung gegenüber, deren jährliche Zuwachsraten im Vergleich mit den bis dahin gemachten Erfahrungen erheblich höher lagen. Zwar ist die Verschuldung der öffentlichen Haushalte insgesamt seit 1960 stets gestiegen (diese Aussage gilt übrigens bis heute) und auf Bundesebene waren auch nur wenige Haushaltsjahre, 1962 sowie 1969 und 1970, durch leichte Netto-Tilgung gekennzeichnet ${ }^{67}$ - eine auffällige Trendveränderung war jedoch nicht zu übersehen. Diese Entwicklung gab den Anstoß zu einer Diskussion über die gesamtwirtschaftlichen Wirkungen öffentlicher Verschuldung und ihre langfristige Tragfahigkeit, die bis heute anhält und die sich u.a. in den Konvergenzkriterien des Maastrichter Vertrages niedergeschlagen hat. ${ }^{68}$ Vor einer detaillierten Untersuchung der Tragfähigkeit der sozial-liberalen Finanz- bzw. Verschuldungspolitik soll im folgenden zunächst ein Überblick über die Defizit- und Schuldenentwicklung skizziert werden.

Betrug die Verschuldung des Bundes ${ }^{69}$ im Jahre 1970 47,8 Mrd. DM, so

67 Auf Landesebene fand von 1961 bis 1963 in einem längeren Zeitraum Netto-Tilgung statt; ansonsten auch nur noch im Jahre 1969. Auf Gemeindeebene findet sich bis auf das Jahr 1974 kein Jahr mit Netto-Tilgung.

Zur neueren Diskussion im Rahmen des Vertrags von Maastricht vgl. etwa Institut „Finanzen und Steuern“ (1993). Dort werden auch die Paralleln zwischen den Konvergenzkriterien gemäß dem Vertrag von Maastricht und den im folgenden noch zu entwickelnden Tragfähigkeitsindikatoren aufgezeigt. Allerdings liegt den Konvergenzkriterien eine andere Abgrenzung der öffentlichen Schuld als die hier verwendete zugrunde.

69 Bund ab 1.1.1980 einschließlich der mitübernommenen Schulden des Lastenausgleichsfonds. $\mathrm{Zu}$ den im folgenden aufgefürten Daten vgl. Statistisches Bundesamt (1994b, 1995) sowie Sachverständigenrat zur Begutachtung der gesamtwirtschaftlichen Entwicklung (1996), sofern keine andere Quelle angegeben ist. Die Wachstumsraten und Quoten basieren auf eigenen Berechnungen. 
stieg sie bis zum Ende der 70er Jahre auf 201,5 Mrd. DM an. Dies entspricht einer Steigerung von rd. 322\%. Dagegen ist der Anstieg von 26,2 Mrd. DM im Jahre 1960 auf 50,3 Mrd. DM 1969 mit 92\% - bezogen auf ein wesentlich niedrigeres Ausgangsniveau - noch vergleichsweise moderat. Während sich die Schuldenquote von 8,7\% 1960 auf 8,4\% 1969 verringerte, stieg sie in den 70er Jahren um 6,1 Prozentpunkte auf 14,5\% 1979 und bis 1982 um weitere 4,9 Punkte auf einen Wert von 19,4\%. Der Schuldenstand hatte 1982 ein Niveau von 308,5 Mrd. DM erreicht. Der Anstieg der Verschuldung hielt jedoch auch nach 1982 an, wenngleich Verschuldungsniveau und Schuldenquote aufgrund der Konsolidierungsmaßnahmen in den Jahren 1982 bis 1989 zunächst langsamer wuchsen (1983: 341,6 Mrd. DM bzw. 20,4\% des BSP; 1989: 490,5 Mrd. DM bzw. 21,8\% des BSP). Erst durch den Aufbau der neuen Bundesländer über den Fonds "Deutsche Einheit" erhielt sie neuen Auftrieb. Bis Ende 1995 erreichte die Verschuldung des Bundes damit ein Niveau von 754,3 Mrd. DM, was einer Schuldenquote von $21,9 \%$ entspricht. Rechnet man die Sonderfonds ebenfalls dem Bund $\mathrm{zu}$, dann wurde sogar ein Niveau von 1.285,1 Mrd. DM bzw. 37,3\% erreicht.

Die Entwicklung der Verschuldung und die dahinterstehenden Haushaltsdefizite sind das Ergebnis einer Fülle von Einflußfaktoren politischer und konjunktureller Natur. Betrachtet man den gesamten Zeitraum von Beginn der 60er Jahre bis heute, so läßt sich nicht übersehen, daß die sozial-liberale Koalition mit einer mehr als Versechsfachung des Schuldenstandes von 1969 bis 1982 und einer mehr als verdoppelten Schuldenquote wesentlich zur Expansion der öffentlichen Verschuldung beigetragen hat. Allgemein reflektiert diese Entwicklung die Reformpolitik der SPD/FDP-Koalition einerseits und die damals keynesianisch geprägte Konjunkturpolitik andererseits. Die speziellen Ursachen variierten jedoch im Laufe der 13-jährigen Regierungszeit.

In den Jahren 1969 bis 1973 nahm der Schuldenstand um 6,9 Mrd. DM bzw. $13,7 \%$ und damit noch vergleichsweise gering zu. Durch die hohe Aus- und streckenweise Überlastung des Produktionspotentials in diesen Jahren war eine Zurückhaltung bei der Neuverschuldung konjunkturpolitisch geboten. Gleichzeitig flossen konjunkturbedingt die Einnahmen so reichlich, daß eine verstärkte Kreditaufnahme nicht erforderlich war. Im Zuge der sozialpoliti- 
Schaubild I

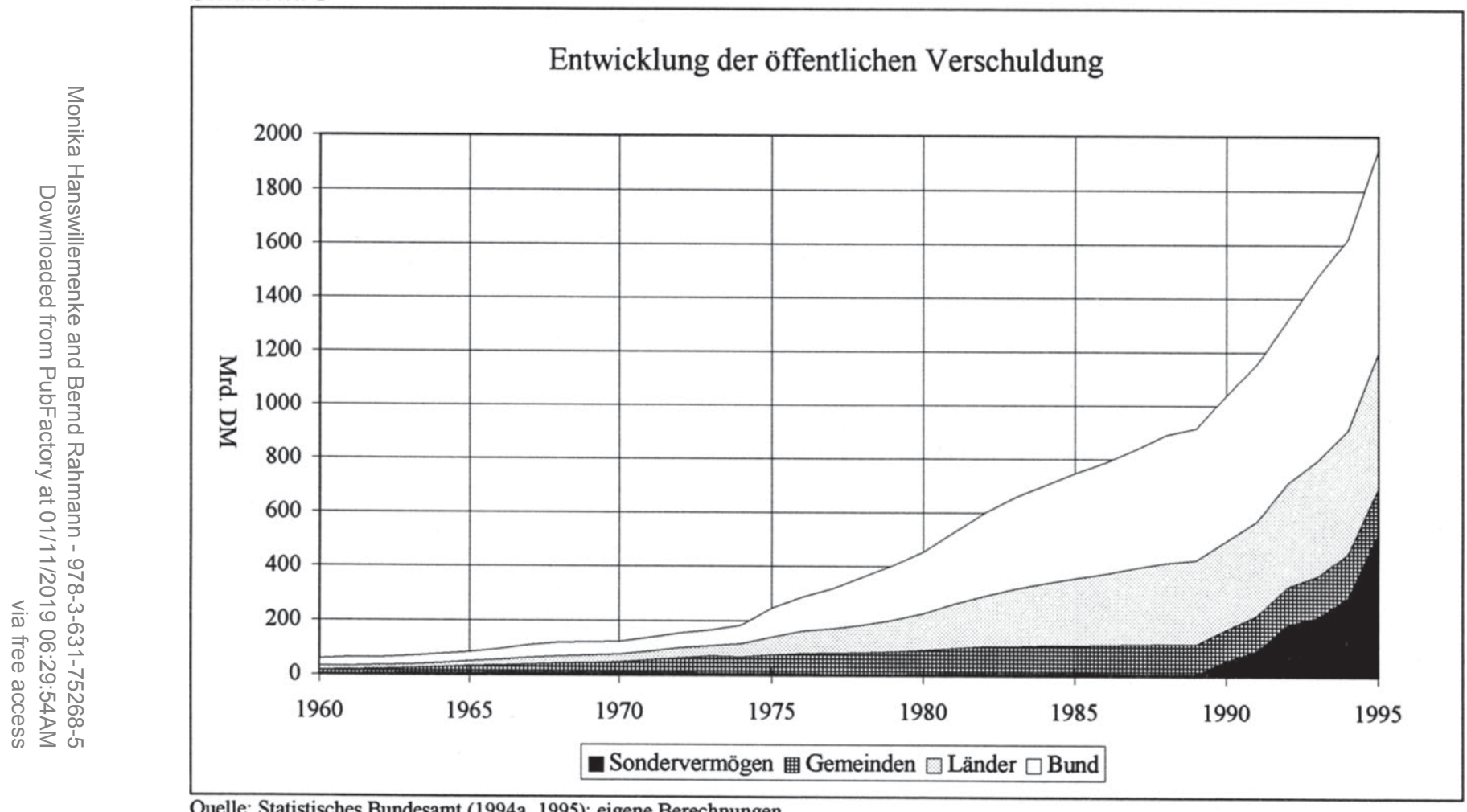


schen Reformen wurde insbesondere zu Beginn der 70er Jahre ein Katalog der staatlichen Aufgaben ausgedehnt. Die hiermit verbundenen höheren Ausgaben, insbesondere verursacht durch Personal und Investitionen, mußten in den Jahren 1970 bis 1973 aufgrund der hohen Einnahmen jedoch nur mit durchschnittlich 2,6 Mrd. DM pro Jahr kreditfinanziert werden.

Die Steuereinnahmen flossen auch deshalb kräftig, weil durch die Inflation die Einkommensbezieher in höhere Progressionsstufen des Steuertarifs gerieten (sog. kalte Progression). Eine Steuerreform zur Entlastung der Steuerpflichtigen war damit auf mittlere Sicht unausweichlich. Da sich jedoch im gleichen Zeitraum die von den öffentlichen Haushalten nachgefragten Güter im Vergleich zu anderen Gütern überdurchschnittlich verteuerten, entstand eine „strukturelle Deckungslücke“, die durch die Steuer- und Kindergeldreform 1975 und den zeitlich damit zusammenfallenden Beschäftigungsrückgang vollends zu Tage gefördert wurde.

Nach 1973 verschlechterte sich die Finanzlage des Bundeshaushaltes erheblich. Da sich seit Ende 1973 ein Rückgang des Wirtschaftswachstums abzeichnete, mußte der bisherige konjunkturpolitische Kurs geändert und sukzessive auf Expansion umgestellt werden. Die 1974 und 1975 beschlossenen Konjunkturprogramme wie das „Sonderprogramm zur regionalen und lokalen Abstützung der Beschäftigung“ (Volumen: 950 Mio. DM), das „Programm zur Förderung von Beschäftigung und Wachstum bei Stabilität" (Volumen: 1,73 Mrd. DM) oder das „Programm zur Stärkung von Bau- und anderen Investitionen“ (Volumen: 5,8 Mrd. DM) konnten aus der bei der Bundesbank angesammelten Konjunkturausgleichsrücklage finanziert werden. ${ }^{70}$ 1975 erlebte die Bundesrepublik jedoch die bis dahin schwerste Rezession der Nachkriegszeit. Das reale Bruttosozialprodukt (gemessen in Preisen von 1991) schrumpfte um 19,4 Mrd. DM, was einer Wachstumsrate von -1,1\% entsprach. Die hohen konjunkturbedingten Mindereinnahmen und Mehrausgaben ließen die Nettoneuverschuldung des Bundes auf 37,7 Mrd. DM an-

70 Vom 09.05. bis 30.11.1973 wird eine Investitionsteuer und vom 01.07.1973 bis 30.04.1974 wird ein Stabilitätszuschlag zur Einkommen- und Körperschaftsteuer erhoben, deren Aufkommen im Sinne des $\S 5$ StabWG bei der Bundesbank zunächst stillzulegen war. 
schwellen. ${ }^{71} \mathrm{Zu}$ dem hohen Niveau von Defizit und Neuverschuldung trug auch die schon genannte Steuerreform sowie die Umstellung des die Steuereinnahmen vermindernden Kinderfreibetrages auf das die Staatsausgaben erhöhende Kindergeld bei. Beide Maßnahmen zusammen schlugen (bei den Gebietskörperschaften insgesamt) mit rd. $18 \mathrm{Mrd}$. DM zu Buche. ${ }^{72}$

Unter dem Eindruck des hohen Schuldenstandes und der Befürchtung, daß die - trotz des seit Mitte 1975 zu verzeichnenden Aufschwungs - angespannte Arbeitsmarktlage auch weiterhin zu hohen finanziellen Belastungen des Haushalts führen könnte, wurde Anfang 1976 das Haushaltsstrukturgesetz verabschiedet mit dem Ziel einer Konsolidierung der öffentlichen Finanzen. Neben dem Sanierungsprogramm und der guten konjunkturellen Lage trugen unerwartete Steuermehreinnahmen zu einer raschen Rückführung des Defizits auf 28,6 Mrd. DM bei. Dieser Trend hielt auch 1977 an; das Defizit belief sich nunmehr auf 22,7 Mrd. DM. Die am 01.01.1977 in Kraft getretene Körperschaftsteuerreform sowie die Erhöhung der Tabak-, der Branntwein- und der Mehrwertsteuer leisteten hierzu ebenfalls einen Beitrag. Im Laufe des Jahres wurde eine expansivere Ausrichtung der Finanzpolitik notwendig, da das Wachstum des Sozialprodukts schwächer ausfiel als erwartet. Nachdem bereits im März ein mehrjähriges „Programm für Zukunftsinvestitionen“ (ZIP) im Umfang von $16 \mathrm{Mrd}^{\text {“ }} \mathrm{DM}^{73}$ beschlossen worden war, traf die Regierung im September weitere wachstums- und beschäftigungsfördernde Maßnahmen, die sich vor allem im „Gesetz zur Steuerentlastung und Investitionsförderung" niederschlugen. Diese Maßnahmen wurden jedoch überwiegend erst im Folgejahr kassenwirksam.

1978 kam es damit konjunkturbedingt zu einer Unterbrechung des Konsolidierungsprozesses. Hinzu kam, da $B$ die deutsche Delegation auf dem Bonner Wirtschaftsgipfel im Juli dieses Jahres zugesagt hatte, als Beitrag zur Abwehr der weltweiten Störungen des wirtschaftlichen Gleichgewichts Maßnahmen im Umfang von bis zu 1\% des Sozialprodukts zu treffen. Diese $\mathrm{Zu}$ -

71 Das Haushaltsdefizit betrug lediglich $35 \mathrm{Mrd}$. DM; die überschüssige Nettokreditaufnahme wurde in das folgende Haushaltsjahr übernommen.

72

73 gl. Deutsche Bundesbank (1979b), S. 16.

Als das Programm 1980 abgewickelt wurde, war ein Gesamtinvestitionsvolumen von 20 Mrd. DM erreicht worden; vgl. Bundesministerium der Finanzen (1980), S. 24 . 
sage war im Kontext der seinerzeit diskutierten sog. „Lokomotivfunktion“ für solche Volkswirtschaften zu sehen, die im Rahmen der Weltwirtschaft eine bedeutsame Rolle spielen. ${ }^{74}$ Zur Realisierung der eingegangenen Verpflichtung wurden im gleichen Monat neben ausgabenwirksamen Maßnahmen u.a. Steuererleichterungen bei der Einkommen- und der Gewerbesteuer beschlossen, die überwiegend 1979 und teilweise in 1980 wirksam wurden. $^{75}$

Der expansive Kurs der Finanzpolitik wurde nicht zuletzt wegen des zweiten Ölpreisschocks und der dadurch ausgelösten Rezession 1980 vorerst beibehalten. 1981 erreichten Defizit bzw. Nettoneuverschuldung ein Niveau von 38 bzw. 39 Mrd. DM; bezogen auf die Regierungszeit der sozial-liberalen Koalition ist dies der Höchststand. Konsolidierungsmaßnahmen waren daher auf Dauer unumgänglich. 1981 leitete die Bundesregierung folgerichtig ein umfangreiches Sanierungsprogramm ein. Für den Haushalt 1982 waren neben Einnahmeerhöhungen insbesondere drastische Ausgabenkürzungen vorgesehen. So konnte trotz eines schrumpfenden Bruttosozialprodukts (die Wachstumsrate betrug real $-1,1 \%$ ) und der daraus resultierenden geringen Steuereinnahmen das Defizit 1982 auf einem Niveau von 37,7 Mrd. DM gehalten werden.

Der allgemeine Anstieg der Verschuldung vollzieht sich bei den Gebietskörperschaften mit unterschiedlichem Tempo. Während die Defizit- und Schuldenentwicklung der Länder in den 70er Jahren anteilmäßig etwa ähnlich zu der des Bundes verlief, entwickelte sich die Verschuldung der Gemeinden, die absolut ebenfalls zunimmt, relativ gegenläufig. Der Anteil der Länder an der Verschuldung der Gebietskörperschaften (einschließlich Sondervermögen), der bereits seit Mitte der 60er Jahre angestiegen war, nahm somit in den 70er Jahren weiter zu (1960: 26,8\%; 1965: 20,1\%; 1970: 22,3\%; 1980: 29,8\%). Der Anteil der Gemeinden war hingegen bis 1973 gestiegen, dann aber kontinuierlich zurückgegangen (1960: 20,4\%; 1973: 37,6\%; 1980: $19,0 \%$ ). Die Gemeinden waren wegen der geringeren Verschuldungsmöglichkeiten am Kapitalmarkt nicht in der Lage, ihre Ausgaben übermäßig aus-

74 Im Überblick Jarchow/Rühmann (1994), S. 111, siehe auch Lehment (1988), S. $160 \mathrm{ff}$.

Vgl. Deutsche Bundesbank (1978a), S. 17. 


\section{Schaubild 2}

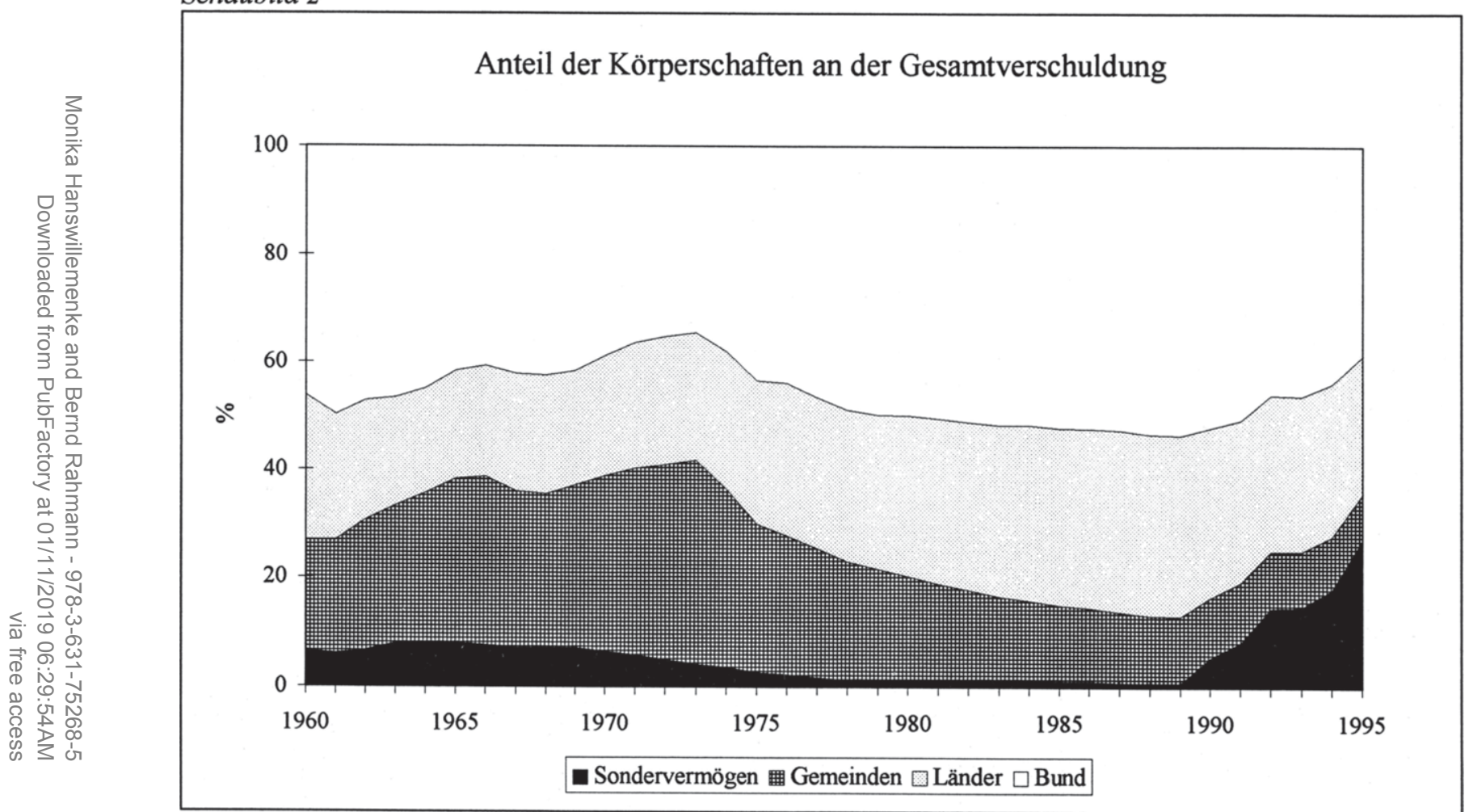


zuweiten. Zwar wurde mit der Reform von 1974 der im Stabilitätsgesetz enthaltene Auftrag, den „Erfordernissen des gesamtwirtschaftlichen Gleich gewichts" Rechnung zu tragen, in die Gemeindeordnungen aufgenommen, die haushaltsrechtlichen Vorschriften orientieren sich gleichwohl überwiegend an einzelwirtschaftlichen Kriterien. ${ }^{76}$ Aufgrund der wachsenden Verschuldung der zentralen Gebietskörperschaften und der relativen Zurückhaltung der Gemeinden war der Anteil der Gemeinden an der gesamten Verschuldung bis Ende der 70er Jahre auf ein Niveau gesunken, das dem zu Beginn der 60er Jahre entsprach.

Bund und Länder haben nicht nur einen weit besseren Zugang zu den Kapitalmärkten, sie tragen auch die Verantwortung für die konjunkturelle Steuerung nach dem Stabilitäts- und Wachstumsgesetz. Hieraus ergaben sich automatisch höhere finanzielle Belastungen, die insbesondere der Bund $\mathrm{zu}$ tragen hatte. So fielen 59\% der Neuverschuldung des Gesamthaushalts in der Rezession 1975 allein auf den Bund. Dort wurde zum erstenmal die Ausnahmeregel des Art. 115 GG angewendet, nach der die Kreditaufnahme des Bundes „zur Abwehr einer Störung des gesamtwirtschaftlichen Gleichgewichts" die Summe der im Haushaltsplan veranschlagten Ausgaben für Investitionen überschreiten darf. Neben den Konjunkturprogrammen gab es jedoch eine Reihe weiterer Maßnahmen, die insbesondere den Bund finanziell belasteten. So war der Bund von der Steuer- und Kindergeldreform 1975 in höherem Maße betroffen als die nachgeordneten Gebietskörperschaften. Hinzu kamen wachsende internationale Verpflichtungen im $\mathrm{Zu}-$ sammenhang mit der Europäischen Integration. All dies hat dazu geführt, daß der Anteil des Bundes an der Gesamtverschuldung, der bis 1973 tendenziell gesunken war, ab Mitte der 70er Jahre fast stetig zunahm und Ende der 70er/Anfang der 80er Jahre rund die Hälfte der Gesamtverschuldung in Anspruch nahm. ${ }^{77 / 78}$

76 Vgl. Deutsche Bundesbank (1979b), S. 19 f.

77 Die Entwicklung setzt sich im Prinzip bis heute fort. Lediglich der wachsende Anteil der Sondervermögen durch die deutsche Einheit hat die Anteile von Bund und Ländern wieder zurückgehen lassen (vgl. Schaubild 2).

Zur beschriebenen Entwicklung vgl. Schlesinger/Weber/Ziebarth (1993), S. 10-18, Deutsche Bundesbank (1973b), Deutsche Bundesbank (1979b), Deutsche Bundesbank (1981b). 


\subsection{Verschiebungen in der Struktur des Haushalts}

Bei der Beschreibung der Defizit- und Schuldenentwicklung wurden strukturelle Aspekte der Haushaltsentwicklung vernachlässigt bzw. nur am Rande erwähnt. Änderungen in der finanzpolitischen Ausrichtung kommen aber vor allem auch in der unterschiedlichen Gewichtung von Allokations-, Distributions- und Stabilisierungspolitik und damit in der Haushaltsstruktur zum Ausdruck. Im folgenden stellt sich daher die Frage, ob - und wenn ja wie sich die Veränderungen in der Finanzpolitik in strukturellen Verschiebungen innerhalb des Budgets niedergeschlagen haben. ${ }^{79}$

\subsubsection{Entwicklung der Ausgabenstruktur}

Zunächst ist festzustellen, daß sich das Ausgabenwachstum im Durchschnitt der Jahre 1976 bis 1982 im Vergleich zu den Jahren 1970 bis 1975 auf nicht einmal zwei Drittel reduziert hat (6,6\% gegenüber $10,9 \%)$. Dabei sind die Wachstumsraten der Jahre 1971 und 1975 korrigiert um die Effekte, die sich aus der Ausgliederung der EG-Finanzierung bzw. der Neuregelung des Familienlastenausgleichs ergeben. War der Anteil der Ausgaben der laufenden Rechnung zunächst rückläufig, so ist für die Jahre 1974 bis 1982 ein nicht kontinuierlicher, aber tendenzieller Anstieg zu verzeichnen. Der Anteil der Ausgaben der Kapitalrechnung hat sich entsprechend gegenläufig entwikkelt. Für den wachsenden Anteil der laufenden Rechnung in der zweiten Phase der Regierungsperiode sind vor allem die Zinsausgaben verantwortlich.

Den größten Anteil an den Gesamtausgaben nehmen bei der Gliederung nach volkswirtschaftlichen Arten die laufenden Zuweisungen und Zuschüsse an andere Bereiche ein. Dazu zählen Übertragungen an den Unternehmens-

79 Die im folgenden verwendeten Daten beruhen im wesentlichen auf den Tabellen 1-8 und 10-13 im Tabellenteil des Finanzberichts 1996. Z.T. wird auch auf die Zusammenstellungen der Ausgaben und Einnahmen der Gebietskörperschaften im Textteil der Finanzberichte 1972 bis 1984 zurückgegriffen. Da im Zeitablauf Änderungen in der Abgrenzung der Daten vorgenommen wurden, wird jedoch - soweit möglich die jüngere Quelle zugrundegelegt. Neuerliche im Finanzbericht 1997 festzustellende Änderungen gegenüber den Vorjahren wurden jedoch nicht berücksichtigt. 
sektor, Renten, Unterstützungen u.a. sowie Zahlungen an Sozialversicherungen, private Organisationen ohne Erwerbscharakter, an die ehemalige DDR und das Ausland. In den Jahren bis 1974 ist der Anteil der Zuschüsse tendenziell gesunken, 1975 ist jedoch ein Anstieg von 35,7 auf 39,7\% festzustellen, und in den folgenden Jahren bleibt der Anteil auf diesem Niveau. Der Durchschnitt der Jahre 1975 bis 1982 beträgt ebenfalls rund 39\%. Demgegenüber fallen die Steigerungsraten dieser Ausgabenkategorie vergleichsweise unterschiedlich aus. Sie variieren zwischen 1,4\% 1977 und 30,0\% 1975. Im Vergleich zum Wachstum der Gesamtausgaben läßt sich keine Systematik erkennen. Die Zuschüsse wachsen teils über-, teils unterproportional, ohne daß sich hieraus ein Trend ablesen ließe. Innerhalb der Transfers nehmen die Übertragungen an den Sozialversicherungsbereich den größten Part ein, gefolgt von der Kategorie Renten, Unterstützungen u.a., wobei für beide ab 1975 der Anteil an den Gesamtausgaben gestiegen ist. Tendenziell gestiegen ist auch der Anteil der Zuweisungen und Zuschüsse an öffentliche Unternehmen. Die Transfers an private Unternehmungen haben hingegen tendenziell abgenommen, ebenso die Übertragungen an das Ausland. Die Niveauverschiebung im Anteil der Renten, Unterstützungen u.a. ist aber insbesondere auf die Kindergeldreform zurückzuführen, nach der ein Teil der bis dahin steuerlichen Entlastung durch Kinderfreibeträge durch das damals neue Kindergeld ersetzt wurde.

Die nächstgrößten Ausgabenkategorien sind die Personalausgaben sowie der laufende Sachaufwand. Zusammengenommen liegen sie knapp unter dem Anteil der Transfers (an andere Bereiche). Der Anteil der Personalausgaben hat sich zwischen 1969 und 1974 von 15,6 auf 17,9\% erhöht und ist danach kontinuierlich bis auf 14,1\% 1982 gesunken. In den Jahren 1970 bis 1974 liegen die Veränderungsraten zwischen 11,4 und $17 \%$ und damit überwiegend oberhalb des Wachstums der Gesamtausgaben. In den folgenden Jahren bis 1982 liegen die Steigerungsraten zwischen 0,9 und 6,5\%, in der Regel unterhalb derjenigen der gesamten Ausgaben. Hier sind in der ersten Phase der Regierungszeit also höhere Werte zu verzeichnen als in der zweiten. Die höchste bzw. niedrigste Rate wird 1971 bzw. 1982 realisiert. Der Anteil der Sachausgaben ist demgegenüber über den gesamten Zeitraum tendenziell gesunken, wobei der Rückgang zur Gänze auf die ersten Jahre des Betrachtungszeitraums entfällt, während der Anteil in den Jahren 1975 bis 1982 relativ konstant bleibt. Die höchsten Veränderungsraten verzeichnet 
der laufende Sachaufwand in den Jahren 1972 bis 1974 (sowie 1978). Auch hier ist das Wachstum - mit Ausnahme von 1970, wo ein negatives Wachstum von 2,3\% zu verzeichnen ist - vor 1975 im Schnitt höher als in den Jahren danach.

Während der Anteil der laufenden Zuschüsse an andere Bereiche sowie die Sachaufwendungen in den Jahren 1975 bis 1982 relativ konstant geblieben sind und der Anteil der Personalausgaben tendenziell gesunken ist, hat der Anteil der Ausgaben der laufenden Rechnung insgesamt jedoch zugenommen. Die Ursache liegt in den gewachsenen Zinsausgaben. Seit 1972 ist ihr Anteil kontinuierlich angestiegen - von 2,5 auf $9 \%$. Sie verzeichnen auch über den gesamten Zeitraum betrachtet - die höchsten Wachstumsraten mit durchschnittlich 19,7\%. Der Durchschnitt der Jahre 1975 bis 1982 liegt sogar bei 23,1\%. Mit Ausnahme der Jahre 1971 und 1972 sind die Zinsausgaben stärker gewachsen als die Gesamtausgaben. In den Jahren 1979 bis 1982 - mit im Vergleich zum Gesamtzeitraum gesunkenem Ausgabenwachstum insgesamt - beträgt das Zinsausgabenwachstum das Zweieinhalbbis Viereinhalbfache des Gesamtausgabenwachstums.

Die Ausgaben der Kapitalrechnung machen zusammengenommen eine GröBenordnung aus, die den Personalausgaben oder dem laufendem Sachaufwand entspricht. Den größten Anteil hierbei nahmen zu Beginn der Regierungsperiode der SPD/FDP-Koalition die Sachinvestitionen ein. Ihr Anteil ist jedoch seit 1971 kontinuierlich gesunken (von 6,5 auf 2,8\% der Gesamtausgaben). Zwar ist dieser Rückgang z.T. auf die Zuwächse bei den anderen Ausgabearten zurückzuführen, für die Jahre 1976 bis 1982 ist jedoch auch ein überwiegend unterproportionales oder negatives Wachstum festzustellen. Die Wachstumsraten der Sachinvestitionen sind relativ variabel mit den höchsten Raten von 12,3\% und 13,4\% in 1970 und 1971 und den niedrigsten von $-10,3 \%$ und $-11,3 \%$ in 1976 und $1981 .^{80}$ In der Entwicklung läßt sich kein klares antizyklisches Verhalten erkennen. Lediglich 1974/75 sind die Ausgaben für Sachinvestitionen entsprechend erhöht worden mit einer Wachstumsrate, die aber unterhalb des Gesamtausgabenwachstums liegt

80 Die Ursache für das negative Wachstum der Sachinvestitionen 1981 lag u.a. in den im Vergleich zum Trend - hohen Investitionsausgaben 1980, die noch auf das „Programm für Zukunftsinvestitionen“ (ZIP) zurückgingen, vgl. Deutsche Bundesbank (1988). 
bzw. mit diesem ungefähr übereinstimmt. Vielmehr drücken die hohen Steigerungsraten zu Beginn den von der Regierung gesehenen Nachholbedarf an öffentlichen Investitionen aus. Da jedoch die Sachinvestitionen zu den Ausgaben gehören, die nicht durch Leistungsgesetze (wie die Zuschüsse) oder (andere) längerfristige Bindungen (wie beispielsweise bei den Personalausgaben) festgelegt sind, sind sie, als Ausgabenkürzungen notwendig wurden, stärker als andere Ausgaben beschnitten worden. Zudem erreichten in einigen Bereichen die Investitionen einen gewissen Sättigungsgrad. So war der Bedarf an Investitionen im Verkehrswesen nach der Wiederaufbauphase überwiegend gedeckt. Hinzu kam, daß Straßenbauprojekte auf einen wachsenden Widerstand der Öffentlichkeit stießen. Die Investitionen im Bereich „Bildung, Wissenschaft, Kultur“ waren ebenfalls rückläufig, was vor allem mit demographischen Veränderungen zusammenhängt. ${ }^{81}$ Die Entwicklung seit 1976 macht auch deutlich, daß die durch die stärkere Angebotsorientierung beabsichtigte Belebung privater Investitionen nicht durch entsprechende öffentliche Investitionen durch den Bund unterstützt worden ist. Vielmehr sind die Wachstumsraten geringer geworden, mit negativen Raten 1976, 1981 und 1982.

Das eher prozyklische Entwicklungsmuster der Sachinvestitionen findet sich ähnlich bei den Vermögensübertragungen an Verwaltungen, die fast vollständig auf Zuweisungen und Zuschüsse für Investitionen entfallen. Nach anfänglichem Anstieg ihres Anteils an den Gesamtausgaben bis 1974, der sich aus dem beschriebenen Nachholbedarf ergab, läßt sich für 1975 bis 1982 ein kontinuierlicher Rückgang - noch unter das ursprüngliche Niveau von 1969 - feststellen. Die Wachstumsraten der Zuweisungen und Zuschüsse an Verwaltungen zeigen ein prozyklisches Verhalten. Die Vermögensübertragungen an andere Sektoren sind im Gegensatz zur Entwicklung der Sachinvestitionen und den Vermögensübertragungen an Verwaltungen anteilmäßig tendenziell gestiegen. Bemerkenswert hierbei sind die hohen Wachstumsraten der Jahre 1977 bis 1979 bei den Zuweisungen und Zuschüssen an Investitionen, die auch hier den überwiegenden Teil ausmachen (1977 und 1979 jeweils 34,8\%, 1978 42,7\%). Diese Zunahme ist nicht zuletzt auf das mehrjährige „Programm für Zukunftsinvestitionen“ (ZIP) zu-

81 Vgl. Deutsche Bundesbank (1972b), Deutsche Bundesbank (1984), Deutsche Bundesbank (1988). Zur Problematik der Ausgabenflexibilität vgl. Ewringmann (1975). 
rückzuführen. Der von der Regierung in der Anfangsphase konstatierte Nachholbedarf schlägt sich ebenfalls in hohen Wachstumsraten der $\mathrm{Zu}$ weisungen und Zuschüsse für Investitionen nieder. Die höchste Variabilität weist mit ihren häufigen Vorzeichenwechseln und Veränderungsraten zwischen -39,3 (1976) und 53,6\% (1975) die Rubrik Darlehensgewährung, Erwerb von Beteiligungen, Kapitaleinlagen auf. Ihr Anteil ist tendenziell gesunken (1969: 4,6\%, 1982: 3,2\% an den Gesamtausgaben). Insgesamt ist der Beitrag der eher ,investiven“ Ausgaben gesunken (14,2\% im Durchschnitt der Jahre 1976 bis 1982 im Gegensatz zu durchschnittlich 17,7\% in den Jahren 1970 bis 1975), während der Anteil der „,konsumtiven“ Ausgaben zugenommen hat. Dabei sind die investiven Ausgaben der Jahre 1978 bis 1980, bedingt durch die erheblichen Ausgaben im Rahmen des ZIP, mit durchschnittlich $15,2 \%$ vergleichsweise hoch.

Bei Gliederung nach Aufgabenbereichen nehmen die Bereiche „Allgemeine Dienste" und „Soziale Sicherung, soziale Kriegsfolgeaufgaben, Wiedergutmachung" mit jeweils rund einem Drittel die größten Anteile an den Gesamtausgaben ein. Die Ausgaben für allgemeine Dienste haben sich im Zeitablauf rückläufig entwickelt, was vor allem auf die gesunkenen Verteidigungsausgaben zurückzuführen ist, die den größten Teil ausmachen. Von 1969 bis 1982 ist der gesamte Bereich von einem Anteil von $31,7 \%$ auf $25,2 \%$ gesunken.

Der Bereich der sozialen Sicherung ist demgegenüber im Betrachtungszeitraum gestiegen, von 30,2\% 1969 auf $35,5 \%$ 1982. Für diese Steigerung sind u.a. die Zahlungen an die Sozial-, einschließlich Arbeitslosenversicherung, die allein schon fast die Hälfte hiervon ausmachen, und die Arbeitsmarktpolitik verantwortlich. Bei beiden ist Mitte der 70er Jahre eine Niveauverschiebung festzustellen. Die gestiegenen Ausgaben für die soziale Sicherung stehen also in engem Zusammenhang mit den gewachsenen Arbeitsmarktproblemen. In den Ausgaben für Arbeitsmarktpolitik, zu denen die Arbeitslosenhilfe, Ausgaben für Berufsausbildung, Fortbildung, Umschulung und sonstige Anpassungsmaßnahmen und produktive Arbeitsförderung zählen, schlagen sich die arbeitsmarktpolitischen Maßnahmen vom November 1976 und Mai 1977 nieder. Es läßt sich aber auch ein Anstieg der sozialpolitisch motivierten Ausgaben verzeichnen. Der Anteil der Familien-, Sozial- und Jugendhilfe wuchs von durchschnittlich 3,7\% 1969 bis 1974 auf 8,9\% 1975 
bis 1982. Dieser Zuwachs ist aber insbesondere auf die Kindergeldreform zurückzuführen (s.o.). Der insgesamt ohnehin eher geringe Anteil der Vermögensbildung verzeichnet zunächst einen tendenziellen Anstieg, fällt aber bis 1982 auf ein Niveau unter das von 1969 zurück.

Im Betrachtungszeitraum ebenfalls gestiegen sind die Anteile der Ausgaben für Bildungswesen, Wissenschaft, Forschung, kulturelle Angelegenheiten, für Energie- und Wasserwirtschaft, Gewerbe, Dienstleistungen (die Zunahme geht vor allem auf den Unterbereich Bergbau, verarbeitendes Gewerbe und Baugewerbe zurück), für Wirtschaftsunternehmen, Allgemeines Grundund Kapitalvermögen, Sondervermögen sowie für die Allgemeine Finanzwirtschaft. Bei letzteren verzeichnen neben den Allgemeinen Zuweisungen vor allem die hierzu gehörenden verschuldungsbedingten Ausgaben, d.h. insbesondere die Zinsen, einen Zuwachs. Die Ausgaben für Schulden nehmen 1982 bereits einen Anteil von 9,3\% ein, nach 3,0\% 1969 und 3,6\% 1975. Dagegen sind neben den Anteilen der Ausgaben für Allgemeine Dienste vor allem die für Ernährung, Landwirtschaft und Forsten sowie Verkehrs- und Nachrichtenwesen zurückgegangen.

Bei den Ländern und Gemeinden lassen sich ähnliche Tendenzen erkennen. Auch hier sind die Anteile der Personalausgaben (bei den Ländern machen diese den größten Anteil an den Ausgaben aus), der laufenden Zuschüsse und der Zinsausgaben tendenziell gestiegen. Der Anteil der Sachaufwendungen bleibt dagegen über den gesamten Zeitraum betrachtet sowohl bei den Ländern als auch bei den Gemeinden nahezu konstant. ${ }^{82}$ Insgesamt ist damit auf beiden Ebenen der Anteil der laufenden Rechnung an den Gesamtausgaben gestiegen. Im Rahmen der Kapitalrechnung nehmen bei Ländern und Gemeinden die Sachinvestitionen den größten Raum ein, wobei der Anteil der Sachinvestitionen an den gesamten Ausgaben bei beiden größer ist als beim Bund. Die Gemeinden allein tätigen mehr als 60\% der Sachinvestitionen der Gebietskörperschaften, mit steigender Tendenz (1982 entfallen mehr als zwei Drittel auf die Gemeinden). Dennoch nehmen die Sachinvestitionen in der Zeit von 1970 bis 1982 bei den Gemeinden wie auch beim Bund und

82 Bei beiden sinkt der Anteil des laufenden Sachaufwands zunächst, erreicht dann aber gegen Ende des Betrachtungszeitraums wieder ein Niveau wie zu Beginn. Änderungen von 1969 auf 1970 sind vernachlässigt, da hierbei die Finanzreform zu stark ins Gewicht fällt. 


\subsection{Verschiebungen in der Struktur des Haushalts}

bei den Ländern einen immer geringeren Teil des Haushalts ein. Dabei zeigt sich in den Wachstumsraten der Sachinvestitionen in den einzelnen Jahren ein eher prozyklisches Verhalten.

Betrachtet man alternativ zu den realisierten Ausgaben die geplanten Werte der mittelfristigen Finanzplanung des Bundes, dann läßt sich auch hier feststellen, daß der Anteil der Investitionen an den Gesamtausgaben zwar zwischenzeitlich gestiegen, über den gesamten Betrachtungszeitraum aber gesunken ist, obgleich als eines der wesentlichen Ziele der mittelfristigen Finanzplanung eine Umstrukturierung des Bundeshaushalts hin zu den investiven und im weiteren Sinne wachstumsfördernden Ausgaben angegeben wird ${ }^{83} \mathrm{Zu}$ den wachstumsfördernden Ausgaben gehören ebenfalls die Ausgaben für Bildung und Ausbildung, Wissenschaft und Forschung. Ihr durchschnittlicher Anteil an den Gesamtausgaben sinkt vom Finanzplan 1971 bis 1975 bis zum Finanzplan 1976 bis 1980 kontinuierlich, steigt dann wieder an und bleibt etwa auf diesem Niveau. ${ }^{84 / 85}$

\subsubsection{Entwicklung der Einnahmenstruktur}

Die Ausgangsbedingungen der sozial-liberalen Koalition waren - gemessen am Finanzierungssaldo des Haushalts - außerordentlich günstig. Die Dekkung der Ausgaben durch ordentliche Einnahmen lag 1969 bei 101,4\%. Der Anteil der durch reguläre Einnahmen finanzierten Ausgaben ist jedoch während der sozial-liberalen Regierungsperiode gesunken und lag 1982 nur noch bei $84,6 \%$, d.h. $15,4 \%$ der Ausgaben mußten 1982 durch Kredite finanziert werden. Die durchschnittliche Deckungsquote lag 1976 bis 1982 bei $85,7 \%$

83 Im Finanzbericht 1977 wird allerdings angezweifelt, daß die Entwicklung der Investitionsausgaben und ihr Anteil an den Gesamtausgaben brauchbare Maßstäbe für den Grad staatlicher Aufgabenerfüllung sind; vgl. Bundesministerium der Finanzen (1976), Finanzbericht 1977, S. 106.

84 Vgl. Institut „Finanzen und Steuern“ (1982), S. 10-19. Zu Investitionen werden hier allerdings neben den Sachinvestitionen auch die Finanzierungshilfen für Investitionsvorhaben Dritter gezählt, d.h. Gewährung von Darlehen, Zuweisungen und Zuschüssen sowie Erwerb von Beteiligungen und Inanspruchnahme aus Gewährleistungen.

Zur mittelfristigen Finanzplanung vgl. auch Abschnitt 3.3.5. 
gegenüber 96,9\% für die Jahre 1970 bis 1974, wobei die im Jahre 1973 stillgelegten Steuereinnahmen nicht mit berücksichtigt sind. Der niedrigste Wert ist 1975 mit 78,9\% zu verzeichnen. Für diese Entwicklung sind sowohl gestiegene Ausgaben als auch ein geringeres Wachstum der Einnahmen verantwortlich.

Die Haupteinnahmequelle des Bundes stellen die Steuern dar. Ihr Anteil an den gesamten Einnahmen ist jedoch in den Jahren zwischen 1969 und 1982 von rund $95 \%$ auf $88 \%$ zurückgegangen. Damit ist der Anteil steuerfinanzierter Ausgaben noch stärker gesunken - von 95,5\% 1969 auf 74,8\% $1982^{86}$ - als der ohnehin rückläufige Anteil ordentlicher Einnahmen an der Finanzierung derselben. Ein Grund hierfür liegt in dem sinkenden Anteil des Bundes an den Verbundsteuern. Weitaus stärker wirkt sich jedoch der wachsende Anteil der EG an den Steuereinnahmen aus, der 1982 3,3\% des gesamten Steueraufkommens beansprucht. 1971, als die EG-Finanzierung erstmals aus dem Bundeshaushalt ausgegliedert wurde, lag der EG-Anteil an den Steuereinnahmen noch bei $0,6 \%$. Die Umverteilung zugunsten von Ländern und Gemeinden und der EG zusammengenommen hat den Anteil des Bundes am Steueraufkommen von 53,9\% 1969 auf 48,4\% 1982 schrumpfen lassen. Dies allein kann jedoch nicht den sinkenden Anteil der Steuern an den Gesamteinnahmen des Bundes erklären, denn bis 1977 lag der Steueranteil bei rund $95 \%$ und nahm erst in den folgenden Jahren tendenziell ab. Dieser Rückgang ist zum Teil in einem geringeren Wachstum des Steueraufkommens begründet. ${ }^{87}$ Die Abnahme von einem Anteil von 93,5\% 1980 um mehr als 5 Prozentpunkte in den beiden folgenden Jahren ist jedoch weniger in den Steuern selbst begründet als in der Entwicklung der sonstigen Einnahmen. Von den sonstigen laufenden Einnahmen nehmen die Einnahmen aus wirtschaftlicher Tätigkeit den größten Anteil ein. Hierzu gehören auch die Abführungen des Bundesbankgewinns an den Bund, die jeweils in dem dem Geschäftsjahr folgenden Jahr haushaltswirksam werden. In den Jahren 1969 bis 1980 wurde in der Regel kein Gewinn abgeführt bzw. der höchste Gewinn lag bei gut einer halben Milliarde DM. 1981 bzw. 1982 wurde jedoch ein Gewinn von rund 2,3 bzw. 10,5 Mrd. DM in den Haushalt eingestellt

86 Ab 1974 Steuern nach Abzug der Ergänzungszuweisungen an Länder.

87 Das Steueraufkommen aller Gebietskörperschaften ist in den Jahren 1978 bis 1982 im Schnitt um 9,6\% gestiegen (ohne 1975 10,8\%), im Vergleich zu durchschnittlich 4,9\% 1970-77. 
(vgl. Tabelle 6, Abschnitt 3.5). Dieser ist für den gewachsenen Anteil der sonstigen Einnahmen an den Gesamteinnahmen von 6,5\% auf 7,6 bzw. $11,7 \%$ und die entsprechende Verringerung des Steueranteils verantwortlich. 1982 machte der Bundesbankgewinn allein schon mehr als $40 \%$ der sonstigen Einnahmen aus und trug mit 4,3\% zur Finanzierung der Ausgaben bei (zum Vergleich: die Anteile des Bundes an der Körperschaft- und der veranlagten Einkommensteuer sowie die Tabaksteuer liegen 1982 mit 10,7, 13 bzw. 12,2 Mrd. DM etwa in der gleichen Größenordnung). Rechnet man den Bundesbankgewinn heraus, dann liegt der Steueranteil weiterhin bei rund $93 \%$.

Die Anteile der Einzelsteuern am gesamten Steueraufkommen unterliegen größeren Schwankungen als die einzelnen Ausgabenkategorien innerhalb der Gesamtausgaben. Dafür sind neben Änderungen im Steuerrecht die Ertragsabhängigkeit bestimmter Steuern, konjunkturell bedingte Veränderungen in der Konsumneigung oder Verschiebungen in der Einkommensverteilung verantwortlich. ${ }^{88}$ Die Anteile der Lohnsteuer und der Körperschaftsteuer am Steueraufkommen des Bundes haben tendenziell zugenommen (1969: 12,1 bzw. 4,9\%; 1982: 28,6 bzw. 5,9\%). Der Anteil der veranlagten Einkommensteuer ist bis Mitte der 70er Jahre ebenfalls gestiegen, dann aber bis 1982 etwa auf das Niveau zu Beginn der Regierungszeit zurückgefallen. Die Anteile der dem Bund zur Gänze zur Verfügung stehenden Mineralöl- und Tabaksteuer haben sich im Laufe der sozial-liberalen Regierung verringert. Der Anteil der Umsatzsteuer (einschließlich Einfuhrumsatzsteuer) liegt 1982 etwa auf dem Niveau von 1970 und unterhalb des Niveaus von 1969. Während der Anteil der gesamten Umsatzsteuer an der Finanzierung der Gebietskörperschaften - vor allem durch die Steuererhöhungen zu Beginn der Jahre 1978 und 1979 um jeweils einen Prozentpunkt - über den Betrachtungszeitraum gestiegen ist, wurde diese Entwicklung beim Bund durch einen sinkenden Anteilssatz z.T. kompensiert. Insgesamt läßt sich feststellen, daß trotz Entlastungen bei der Einkommen-, Vermögen- und Gewerbesteuer und Erhöhungen bei den Verbrauchsteuern der Beitrag der direkten Steuern (in der Abgrenzung der Volkswirtschaftlichen Gesamtrechnung) an der Finan-

88 Auf eine detaillierte Beschreibung und Begründung der Entwicklung der einzelnen Steuern muß hier verzichtet werden. Insoweit Änderungen im Steuerrecht hierfür verantwortlich sind, sei auf den vorhergehenden Abschnitt 1.3 und die dort angegebene Literatur verwiesen. 
zierung des Gesamthaushalts sowie des Bundes über den Zeitraum von 1969 bis 1982 gestiegen ist (Bund: 1970 33,8\%, 1982 42,9\%). Diese Gewichtsverlagerung ist letztlich eine dem deutschen Steuersystem innewohnende Tendenz, die vor allem auf der progressiven Ausgestaltung der Einkommensteuer beruht. Die in Abschnitt 1.2 beschriebene Umstrukturierung der Einnahmenseite hin zu einem investitionsfreundlicheren Steuersystem läßt sich aus der Entwicklung der Steuereinnahmen des Bundes nicht ohne weiteres herauslesen. Am ehesten läßt sie sich im Gesamthaushalt in Form sinkender Finanzierungsbeiträge von Gewerbe- und Vermögensteuer erkennen sowie in einer seit 1977 rückläufigen Steuerquote (Steuereinnahmen in Relation zum Bruttosozialprodukt) $)^{89}$.

Im Gegensatz zum Bund nimmt der Anteil der Länder und Gemeinden am Steueraufkommen tendenziell $\mathrm{zu}$, wobei jedoch die Anteile Mitte der 70er Jahre höher liegen als zu Beginn der 80er Jahre. Insgesamt steigen die Steueranteile von Ländern und Gemeinden von 1970 bis 1982 von 32,8 bzw. $12 \%$ auf 34,8 bzw. 13,5\%. Dennoch nimmt - wie auch beim Bund - der Anteil der Steuern an den Einnahmen (ohne Kreditmittel), der insgesamt schon wesentlich geringer ist als beim Bund, in den Jahren 1970 bis 1982 ab (Länder: 1970 70,6, 1982 67,7; Gemeinden: 1970 32,8, 1982 32,2). Allein rund 12 bzw. 20\% der Länder- bzw. Gemeindeeinnahmen entfallen auf Zuweisungen. 90

89 Steuerquote des Bundes bzw. der gesamten Gebietskörperschaften: 1977 12,1 bzw. $24,4 \%, 198211,6$ bzw. $23,1 \%$.

90 Für eine ausführliche Beschreibung der Entwicklung der Einzelsteuern vgl. Deutsche Bundesbank (1978b). 


\section{Beschäftigungspolitik der sozial-liberalen Koalition}

\subsection{Vorbemerkung}

Der Beschäftigungspolitik der sozial-liberalen Koalition kommt im vorliegenden Kontext deshalb hervorragende Bedeutung zu, weil die Bemühungen zur Wiedererlangung der Vollbeschäftigung (im Sinne des Auftrages aus dem § 1 StabWG) die finanz- und haushaltspolitischen Aktivitäten entscheidend dominiert haben und die zu Anfang der Koalition am Beginn der 70er Jahre zunächst überwiegenden Ausgaben im Rahmen der „Politik der inneren Reformen" dadurch spürbar zurückgedrängt worden sind.

Dabei wird zum besonderen Problem, daß entgegen den bisherigen Erfahrungen die Arbeitslosigkeit in mehrfacher Hinsicht ihren Charakter verändert: Es handelt sich nicht mehr ausschließlich um konjunkturelle, sondern zunehmend um strukturelle Arbeitslosigkeit, wobei die Dauer der durchschnittlichen individuellen Arbeitslosigkeit tendenziell zunimmt, so daB im Endeffekt eine verfestigte Massenarbeitslosigkeit auftritt, die über einen kurzfristig wirkenden Nachfrageimpuls nicht mehr nachhaltig abgebaut werden kann. Das in diesem Zusammenhang damals erstmals auch für die Bundesrepublik beobachtete Phänomen der „Entkopplung von Arbeitsmarkt und Gütermarkt ${ }^{\leftrightarrow 91}$ ist sowohl für die Politik als auch für die Theorie ein neues und noch weitgehend unbekanntes Gebiet. Erst allmählich werden Konzepte der Ergänzung keynesianischer Beschäftigungspolitik um strukturelle Komponenten erarbeitet; von einer abrupten Kursänderung zur angebotsorientierten Arbeitsmarktpolitik kann also keine Rede sein. Wenn trotzdem Erfolge bei der Bekämpfung der Arbeitslosigkeit nach 1975 registriert worden sind, so lag das nur zum Teil an der Übereinstimmung von beschäftigungspolitischer Strategie und Problemstruktur bei der Arbeitslosigkeit. $\mathrm{Zu}$ vermuten ist vielmehr auch, da $B$ die Verminderung der Arbeitslosigkeit bis 1979 das eher ungeplante Ergebnis eines Aufeinandertreffens von faktischen Nachfragewirkungen einer konzeptionell strukturorientierten Angebotspolitik einerseits mit dem Vorhandensein von Teilen keynesianischer Arbeitslosigkeit innerhalb der insgesamt als klassisch eingestuften Unterbeschäfti-

91 Siehe zu diesem als „Okun's Law“ bezeichneten Phänomen im Überblick Bolle (1983) und Reineke (1986). 
gung andererseits ist. Diese Konstellation hat möglicherweise zu einem Fehlschluß geführt: Die sichtbaren Erfolge beim Abbau der Arbeitslosigkeit wurden im Grunde genommen den falschen Instrumenten (nämlich den strukturorientierten) zugerechnet, während tatsächlich die Nachfrageeffekte wirksam wurden. Die zu geringe Wirkung auf die Arbeitslosenquote wurde infolgedessen als ein Dosierungsproblem aufgefaßt und führte zu höheren Einsätzen eben der falschen Instrumente, also dazu, daß eine zumindest für die kurzfristig gegebene Situation wenig erfolgversprechende Politik verstärkt fortgesetzt wurde. Erst der übermächtig werdende fiskalische Streß hat diesem Dilemma ein Ende gemacht. Der Politik ist dieser Vorwurf aber nicht allein zu machen, sind derartige Erkenntnisse doch nicht zur gleichen Zeit formuliert worden wie die Probleme aufgetreten sind. Die nachfolgende Wende zur supply-side auch in der Arbeitsmarktpolitik war denn auch weniger eine Einsicht in die arbeitsmarkttheoretischen Grundlagen einer neuen Beschäftigungspolitik als vielmehr die pauschale Folge einer anderen Arbeitsmarktideologie in der Politik: Es fand schlicht eine politische Verabschiedung von der Vollbeschäftigungsgarantie und eine Verengung des Verständnisses von gesamtwirtschaftlicher Stabilität auf das Preisniveau statt mit dem Verweis darauf, daß Vollbeschäftigung ein Problem der optimalen Steuerungsfähigkeit des Arbeitsmarktes sowie des Verhaltens der Tarifvertragsparteien und nicht der prozeßpolitischen Intervention der Politik sei.

Im folgenden werden die beschäftigungspolitische Konzeption der sozialliberalen Bundesregierung in den 70er Jahren sowie die Entwicklung der Arbeitslosigkeit in dieser Zeit beschrieben, um auf dieser Basis die erfolgte Beschäftigungspolitik näher erklären zu können. 


\subsection{Die beschäftigungspolitische Konzeption}

Mit der Ende der 60er Jahre entstandenen Neukonzeption der Finanz- und Wirtschaftspolitik wurde auch der Rahmen für die Beschäftigungs- bzw. Arbeitsmarktpolitik ${ }^{92}$ neu gesteckt. Grundlagen dieser Politik innerhalb der Gesamtkonzeption waren das Gesetz zur Förderung der Stabilität und des Wachstums der Wirtschaft einschließlich der darin eingebetteten konzertierten Aktion, das Arbeitsförderungsgesetz (AFG) vom 13.05.1969 sowie das Berufsbildungsgesetz vom 14.08.1969 und das Ausbildungsförderungsgesetz 26.08.1971. Stabilitäts- und Wachstumsgesetz und AFG als Hauptpfeiler stehen dabei in einem Komplementaritätsverhältnis. Während das Stabilitätsund Wachstumsgesetz mit seiner globalen Ausrichtung darauf zielte, einen hohen Beschäftigungsstand zu sichern, sollte das AFG durch selektive Maßnahmen eine wachstumsfördernde Beschäftigungsstruktur schaffen sowie zur Vermeidung struktureller Arbeitslosigkeit und zur Abfederung sozialer Anpassungslasten beitragen ${ }^{93}$ Das AFG ergänzt das Stabilitäts- und Wachstumsgesetz gewissermaßen durch ein ,fine tuning" auf dem Arbeitsmarkt. Wesentlich ist hierbei, daß der Schwerpunkt des AFG in einer vorausschauenden und prophylaktischen Politik gesehen wurde - im Gegensatz zu seinem Vorgänger, dem Gesetz über Arbeitsvermittlung und Arbeitslosenversicherung (AVAVG) vom 23.12.1956, dessen Instrumentarium vornehmlich reaktiv und kompensatorisch ausgerichtet war ${ }^{94}$ Ebenso wie sich zu Beginn der 70er Jahre bereits die Grenzen einer Globalsteuerung im Bereich der restriktiven Fiskalpolitik gezeigt hatten, erwies sich das Instrumentarium zur

92 Im folgenden werden die Bereiche Beschäftigungspolitik und Arbeitsmarktpolitik nicht weiter differenziert und unterschieden. Dies entspricht im wesentlichen der Handhabung in Theorie und Praxis Ende der 60er Jahre und Anfang der 70er Jahre. Erst später erfolgt eine deutlichere Abgrenzung der makroökonomischen Beschäftigungsperspektive keynesianischer und der mikroökonomischen Arbeitsmarktperspektive auf Basis der Modellierung spezifischer Teilarbeitsmärkte. Siehe dazu u.a. Biehler/Brandes/Buttler/Gerlach/Liepmann (1981).

Vgl. § 1 AFG, in dem es heißt: „Die Maßnahmen nach diesem Gesetz sind im Rahmen der Sozial- und Wirtschaftspolitik der Bundesregierung darauf auszurichten, $\mathrm{da} ß$ ein hoher Beschäftigungsstand erzielt und aufrecht erhalten, die Beschäftigungsstruktur ständig verbessert und damit das Wachstum der Wirtschaft gefördert wird." Zur näheren Erläuterung der Entstehungsgeschichte und der Entwicklung des AFG, seiner Ziele und Instrumente wie auch der Erfahrungen mit dem Gesetz vgl. Lampert (1989) sowie Kühl (1982). 
globalen Beschäftigungsförderung als überfordert. Inwieweit das Instrumentarium möglicherweise gar nicht geeignet war, die Arbeitslosigkeit zu beseitigen, weil die gewählten Maßnahmen den Ursachen nicht adäquat waren, ist im folgenden zu untersuchen. 


\subsection{Die Entwicklung der Arbeitslosigkeit}

Mit Ausnahme der Rezession 1967/68 stellte die Arbeitslosigkeit in der Bundesrepublik bis 1973 praktisch kein Problem dar. Die nach dem Krieg bestehende Arbeitslosigkeit wurde im Laufe der 50er Jahre fast vollständig abgebaut. In den 60er Jahren lag die Arbeitslosenquote mit Ausnahme der Jahre 1967 und 1968 zwischen 0,7 und 0,9\%. ${ }^{95}$ Der Arbeitsmarkt war gekennzeichnet durch eine Übernachfrage, die durch das Anwerben von Gastarbeitern jedoch abgemildert wurde. ${ }^{96}$ Ein ernsthaftes Problem wurde die Arbeitslosigkeit erst in der Folge der ersten Ölkrise im Herbst 1973. Die Arbeitslosenquote stieg bis 1975 sprunghaft auf $4,6 \%$ an. Trotz umfangreicher Konjunktur- und Beschäftigungsprogramme und obwohl bereits 1976 wieder ein reales Bruttosozialproduktswachstum von 5,5\% zu verzeichnen ist, nahm die Zahl der Arbeitslosen nur langsam ab. 1979/80 lag die Arbeitslosenquote noch bei 3,6\%. Anstelle einer nachhaltigen Verbesserung auf dem Arbeitsmarkt löste der zweite Ölpreisschock einen neuerlichen Anstieg - noch über das Niveau von 1975 hinaus - aus. Im Unterschied zum ersten Ölpreisschock, der die deutsche Wirtschaft am Anfang einer Rezession traf, trat der zweite Ölpreisschock inmitten eines Aufschwungs auf. ${ }^{97}$ Mit einer Quote von 8,8-8,9\% 1983 bis 1985 erreichte die Arbeitslosigkeit einen neuen Höchststand. Erst gegen Ende der 80er Jahre sank die Arbeitslosenquote wiederum. Sie fiel jedoch nicht unterhalb von 6\%, sondern erhielt seit 1992 rezessionsbedingt erneut Auftrieb. ${ }^{98}$ Insgesamt ergibt sich ein treppenförmiger Verlauf der Arbeitslosenquote (vgl. Schaubild 3). Der in der Krise Mitte der 70er und Anfang der 80er Jahre ausgelöste Anstieg der Arbeitslosigkeit konnte im darauffolgenden Aufschwung nicht nachhaltig abgebaut werden.

$95 \mathrm{Zu}$ den hier verwendeten Arbeitslosenquoten siehe Sachverständigenrat zur Begutachtung der gesamtwirtschaftlichen Entwicklung (1996), Tabelle 21*.

96 Die Zahl der Gastarbeiter stieg von 280.000 im Jahre 1960 auf 2,6 Mio. im Jahre 1973 , das ist ein Anstieg von 1,3\% der Beschäftigten auf rund 10\%. In der Rezession 1967/68 fungierten sie als eine Art Puffer. Ihre Zahl ging zunächst zurück, erreichte aber Mitte 1969 wieder das Niveau vor der Rezession, vgl. Franz/König (1986), S. S219 f. Vgl. ebenda, S. S220.

98 Die neuen Bundesländer sind hier nicht mit betrachtet. Nimmt man sie hinzu, dann erhält man bereits ab 1991 wachsende Arbeitslosenzahlen. 
Schaubild 3

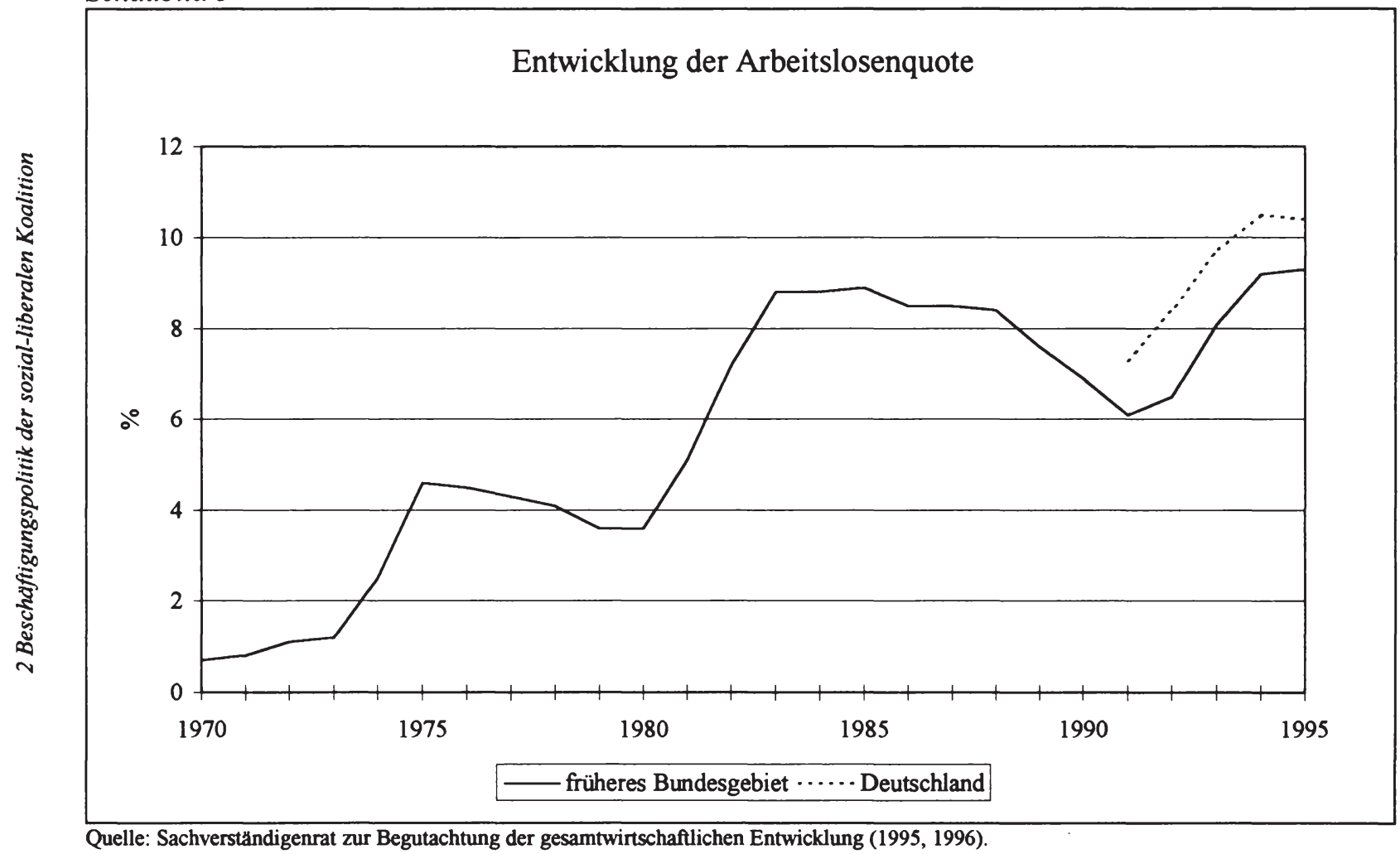


Statt dessen ging die deutsche Wirtschaft jeweils mit einem erhöhten Sockel an Arbeitslosen in den nächsten Abschwung. ${ }^{99}$

Hinter der Entwicklung in den 70er Jahren stand zum einen eine tendenziell gewachsene Anzahl an Arbeitskräften, die u.a. mit einer höheren Beteiligung der Frauen am Arbeitsmarkt sowie einem gestiegenen Anteil ausländischer Arbeitnehmer begründet werden kann. Zum anderen war seit 1973 ein Rückgang der Beschäftigung zu beobachten, der vor allem das verarbeitende $\mathrm{Ge}-$ werbe betraf, während Dienstleistungs- und öffentlicher Sektor einen stetigen Anstieg der Beschäftigten verzeichneten. ${ }^{100}$ Die offiziellen Arbeitslosenzahlen, die lediglich jene Personen erfassen, die sich beim Arbeitsamt als arbeitslos registrieren lassen, geben die Unterauslastung des Arbeitskräftepotentials allerdings nur unzureichend wieder. Nicht erfaßt sind Personen, die sich nicht arbeitslos melden, weil sie ohne Hilfe des Arbeitsamtes einen Arbeitsplatz suchen und/oder keinen Anspruch auf Arbeitslosengeld haben sowie jene, die sich vom Arbeitsmarkt zurückziehen bzw. erst gar nicht in den Arbeitsmarkt eintreten, da sie wegen der schlechten Arbeitsmarktlage keine Chancen auf einen Arbeitsplatz sehen (,entmutigte Arbeitskräfte“ oder „Stille Reserve“). Ebenfalls nicht eingerechnet sind Gastarbeiter, die „unfreiwillig“ in ihre Heimatländer zurückgehen sowie Kurzarbeit und Personen in Umschulungen. Rechnet man diese hinzu, dann erhält man für 1970 eine Unterauslastungsquote von $0,8 \%$, die bis 1973 auf $1,4 \%$ ansteigt. Die Differenzen zur offiziellen Statistik (1970: 0,8\%; 1973: 1,2\%) halten sich somit zunächst noch in Grenzen. Ab 1974 ist jedoch eine deutlich höhere Unterauslastung als sie durch die offizielle Arbeitslosenquote ausgedrückt wird festzustellen. 1974 und 1975 liegt die Unterauslastungsquote bei 4,1 bzw. 8,6\%. Bis 1980 geht sie wieder auf 6,5\% zurück und erfährt 1981 und 1982 einen Anstieg auf 9,4 bzw. 12,7\%, der sich auch im Folgejahr noch fortsetzt. $^{101}$

99 Zu möglichen Erklärungen für dieses als „Hysterese“ oder „Persistenz“ sowie in der mikroökonomischen Arbeitsmarktforschung als „state dependence“ bezeichnete Phänomen vgl. Abschnitt 2.5.

100 Die Bereiche Land- und Forstwirtschaft, Energie, Baugewerbe und Handel und Verkehr verzeichneten ebenfalls einen Rückgang. Vgl. Franz/König (1986), S. S220S224.

101 Vgl. Franz (1985) sowie Franz/König (1986). Schätzungen des IAB gehen von einer Stillen Reserve von 53.000 Personen für die Jahre 1970 bis 1974 sowie 697.000 
Tabelle 1: Arbeitslosigkeit im internationalen Vergleich

Standardisierte Arbeitslosenquoten

in v.H. der gesamten Arbeitskräfte

\begin{tabular}{lrrrrrrrr}
\hline & 1970 & 1973 & 1975 & 1980 & 1982 & 1983 & 1991 & 1995 \\
\cline { 2 - 8 } USA & 4,8 & 4,8 & 8,3 & 7,0 & 9,5 & 9,5 & 6,8 & 5,5 \\
Japan & 1,1 & 1,3 & 1,9 & 2,0 & 2,4 & 2,6 & 2,1 & 3,1 \\
BRD & 0,8 & 0,8 & 3,6 & 2,9 & 5,9 & 7,7 & 4,2 & 8,2 \\
Frankreich & 2,5 & 2,7 & 4,0 & 6,2 & 8,1 & 8,3 & 9,4 & 11,6 \\
Italien & 5,3 & 6,2 & 5,8 & 7,5 & 8,4 & 8,8 & 9,9 & 12,2 \\
GroBbritannien & 3,0 & 3,0 & 4,3 & 6,4 & 11,3 & 12,4 & 8,8 & 8,7 \\
Kanada & 5,6 & 5,5 & 6,9 & 7,5 & 10,9 & 11,9 & 10,3 & 9,5 \\
Australien & 1,6 & 2,3 & 4,8 & 6,0 & 7,1 & 9,9 & 9,5 & 8,5 \\
Belgien & 2,1 & 2,7 & 5,0 & 8,8 & 12,6 & 12,1 & 7,2 & 9,5 \\
Finnland & 1,9 & 2,3 & 2,2 & 4,6 & 5,3 & 5,4 & 7,5 & 17,1 \\
Niederlande & 1,0 & 2,2 & 5,2 & 6,0 & 11,4 & 12,0 & 7,0 & 6,5 \\
Norwegen & 1,6 & 1,5 & 2,3 & 1,6 & 2,6 & 3,4 & 5,5 & 4,9 \\
Spanien & 2,4 & 2,5 & 3,6 & 11,1 & 15,6 & 17,0 & 16,0 & 22,7 \\
Schweden & 1,5 & 2,5 & 1,6 & 2,0 & 3,2 & 3,5 & 3,3 & 9,2 \\
\hline OECD gesamt & 3,1 & 3,3 & 5,2 & 5,8 & 8,2 & 8,6 & 6,8 & 7,5 \\
\hline
\end{tabular}

Anmerkung: BRD bis 1991 früheres Bundesgebiet.

Quelle: OECD $(1990,1996)$.

Diese Entwicklung zu höherer Arbeitslosigkeit vollzieht sich nicht nur in der Bundesrepublik Deutschland. Praktisch alle OECD-Länder haben in den 70er und 80er Jahren einen Anstieg der Arbeitslosigkeit erfahren. Die Länder der EG waren jedoch am stärksten betroffen, wohingegen der Anstieg in den EFTA-Ländern sowie in Japan relativ moderat ausfiel. Die USA verzeichneten ebenfalls Mitte der 70er Jahre und Anfang der 80er Jahre einen Anstieg der Arbeitslosenquoten, der aber - im Gegensatz zur EG - jeweils in der Folgezeit wieder abgebaut werden konnte, so daß sie Ende der 80er Jahre etwa auf dem Niveau zu Beginn der 60er Jahre lagen. Zu Beginn der 60er Jahre hatte die Arbeitslosenquote allerdings über der in der EG gelegen und blieb auch bis Anfang der 80er Jahre noch darüber. Die unterschiedlichen Entwicklungen in den einzelnen Ländern können einen Hinweis auf mögliche Ursachen der anhaltenden Arbeitslosigkeit in der Bundesrepublik

Personen für die Jahre 1975 bis 1979 aus. 1990 bis 1992 liegt die Schätzung bereits bei 1,3 Millionen Personen. Vgl. Franz (1994), S. 330. 
geben. So ist beispielsweise in der Bundesrepublik (wie generell in der EG) ein relativ hoher Anteil Langzeitarbeitsloser festzustellen. ${ }^{102}$ Bevor jedoch auf mögliche strukturelle Ursachen für die Verfestigung der Arbeitslosigkeit in der Bundesrepublik seit Mitte der 70er Jahre eingegangen wird, soll zunächst untersucht werden, inwieweit sich die Arbeitslosigkeit in dem hier interessierenden Zeitraum von 1970 (bzw. 1973) bis 1982 als „klassisch“ oder „keynesianisch“ klassifizieren läßt.

102 Vgl. Layard/Nickell/Jackman (1994), insbesondere S. 1-8 sowie Kapitel 9. 


\subsection{Klassische oder keynesianische Arbeitslosigkeit?}

Es gibt eine Vielzahl von Erklärungsansätzen für Arbeitslosigkeit, auf die im Rahmen dieser Studie jedoch nicht näher eingegangen werden kann. ${ }^{103}$ Schwerpunktmäßig soll im folgenden auf empirisch geschätzte Ungleichgewichtsmodelle zurückgegriffen werden, die eine Klassifikation der Arbeitslosigkeit in sog. „klassische“ oder „keynesianische“ Unterbeschäftigung erlauben. Eine solche Vorgehensweise erscheint insofern sinnvoll, als das Stabilitäts- und Wachstumsgesetz mit seinem Instrumentarium auf eine Bekämpfung keynesianischer Arbeitslosigkeit ausgelegt ist, d.h. auf eine Unterbeschäftigung, die ihre Ursache in mangelnder Nachfrage hat. Eine mögliche Ursache für den langfristig gesehen geringen Erfolg der sozial-liberalen Beschäftigungspolitik könnte darin gelegen haben, daß die Bundesregierung versuchte, mit nachfragestützenden Maßnahmen eine Arbeitslosigkeit zu bekämpfen, die ihre Gründe nicht oder nur zum Teil in einer mangelnden Nachfrage hatte. Zudem lassen sich so Anhaltspunkte dafür finden, wie die in Abschnitt 1.2 beschriebene Abkehr von einer nachfrageorientierten Politik aus beschäftigungspolitischer Sicht zu beurteilen ist. Daneben ist seit Mitte der 70er Jahre eine Reihe von Programmen beschlossen worden, die direkt bei den betroffenen Arbeitslosen ansetzen (z.B. Mobilitäts- und Eingliederungshilfen). Diese Maßnahmen lassen sich nach dem Kriterium klassische oder keynesianische Unterbeschäftigung nicht beurteilen, da es sich hierbei um niveaubedingte, bei aggregiertem Angebot und aggregierter Nachfrage ansetzende Komponenten der Arbeitslosigkeit handelt. Auf die strukturellen Ursachen der Arbeitslosigkeit, an denen diese Programme ansetzen, wird im folgenden Abschnitt kurz eingegangen werden. ${ }^{104}$

Im Rahmen von Ungleichgewichtsmodellen bzw. Modellen mit Mengenrationierung ${ }^{105}$ wird davon ausgegangen, daß in den Regel Ungleichgewichte

103 Einen guten Überblick geben beispielsweise Franz (1994) oder Layard/Nickell/Jackman (1994).

104 Zur Unterscheidung niveaubedingter und strukturbedingter Elemente der Arbeitslosigkeit vgl. Kromphardt (1987).

$105 \mathrm{Zu}$ den folgenden Ausführungen vgl. Benassi/Chirco/Colombo (1994), Felderer/ Homburg (1994), Kapitel XI, Rothschild (1981) sowie Schneider (1987). Auf spezielle Annahmen der im folgenden verwendeten Modelle kann hier nicht näher ein- 
auf den Märkten bestehen und eine vollständige Markträumung nur in Ausnahmefällen gegeben ist. Betrachtet werden im folgenden jeweils nur der Güter- und der Arbeitsmarkt. Ausgangspunkt ist die von Clower so bezeichnete „duale Entscheidungshypothese“. Danach werden die am Wirtschaftsprozeß Beteiligten ihre Entscheidungen revidieren, sobald sie sich am Markt einer wirksamen Beschränkung gegenübersehen. So wird beispielsweise jemand, der am Arbeitsmarkt die von ihm angebotene Arbeit nicht oder nur teilweise absetzen kann, auch seine Konsumpläne anpassen, da ihm ein geringeres als das geplante Einkommen zur Verfügung steht. Die ursprünglich gewünschten Angebots- und Nachfragefunktionen (im Beispiel die Arbeitsangebots- und Güternachfragefunktion) werden durch tatsächlich wirksame (effektive) ersetzt. Die Ursache dafür, daß es zu Tauschaktionen bei „falschen“, d.h. nicht markträumenden Preisen kommt, liegt in der Annahme, daß Preise nicht flexibel genug auf Überschüsse im Angebot oder in der Nachfrage reagieren. Während sich die Mengen quasi sofort anpassen, reagieren die Preise mit einer gewissen zeitlichen Verzögerung. Im Gegensatz zu den ursprünglichen Plänen (der ersten Stufe), die allein auf Preissignalen des Marktes basierten, berücksichtigen die revidierten Pläne (der zweiten Stufe) ebenfalls die Mengensignale (von anderen Märkten). Diese Mengensignale werden als Übertragungs- oder spill-over-Effekte bezeichnet. Im obigen Beispiel überträgt sich die Mengenrestriktion auf dem Arbeitsmarkt auf den Gütermarkt.

Je nachdem, welche Marktseite sich nun einer Mengenbeschränkung gegenübersieht, lassen sich vier Fälle unterscheiden:

- Klassische Unterbeschäftigung: In diesem Fall sind die Haushalte auf Arbeits- und Gütermarkt rationiert. Aufgrund zu hoher Löhne wird das Arbeitsangebot nicht vollständig von den Unternehmungen absorbiert. Die Folge ist Arbeitslosigkeit. Gleichzeitig besteht jedoch am Gütermarkt eine Überschußnachfrage. Spill-over-Effekte treten hierbei nicht auf.

- Keynesianische Unterbeschäftigung: Auf beiden Märkten existiert ein Überangebot. Arbeitslosigkeit führt einerseits zu Konsumeinschränkungen und damit zu einer Rationierung der Unternehmen auf dem Gütermarkt. Mangelnde Nachfrage zieht andererseits Entlassungen nach sich mit der

gegangen werden. Dazu muß auf die entsprechend angegebene Literatur verwiesen werden. 
Folge einer Beschränkung der Haushalte auf dem Arbeitsmarkt. Hier wirken also Übertragungseffekte vom Arbeitsmarkt auf den Gütermarkt und umgekehrt.

- Zurückgestaute Inflation: Hierbei besteht auf beiden Märkten eine Übernachfrage. Fehlende Arbeitskräfte führen zu Produktionsausfällen auf dem Gütermarkt. Das Vorhandensein eines Spill-over-Effekts in umgekehrter Richtung hängt davon $\mathrm{ab}$, ob das unzureichende Konsumgüterangebot $\mathrm{zu}$ einem sinkenden Arbeitsangebot führt oder ob die Bereitschaft zu arbeiten davon unberührt bleibt.

- Unterkonsumtion: Hierbei sind die Unternehmen auf beiden Märkten rationiert. Dieser Fall dürfte jedoch eher ein Übergangsstadium beschreiben, da eine Verringerung der Produktion mit einem entsprechenden Rückgang der Nachfrage nach Arbeitskräften bereits zu einem Gleichgewicht auf beiden Märkten führen kann.

Der Fall eines Walrasianischen Gleichgewichts, d.h. in diesem Fall eine Räumung auf dem Arbeits- und Gütermarkt, ist gewissermaßen ein Grenzfall, in dem keine Marktpartei rationiert wird.

Im folgenden wird insbesondere von Interesse sein, inwieweit sich im Rückblick für den Zeitraum von 1973 bis 1982 keynesianische Arbeitslosigkeit diagnostizieren läßt, denn nur in diesem Falle sind fiskalpolitische Eingriffe (in Form zusätzlicher Staatsausgaben) sinnvoll. In einem solchen Falle wird über eine steigende Produktion die Nachfrage nach Arbeitskräften erhöht. Durch die erhöhte Beschäftigung wird dann die Konsumnachfrage auf dem Gütermarkt angeregt. ${ }^{106}$ Herrscht dagegen klassische Arbeitslosigkeit, dann trifft die zusätzliche staatliche Nachfrage auf ein von der Angebotsseite her restringiertes Güterangebot und führt zu einem (totalen) crowding out. Klassische Arbeitslosigkeit kann nur durch eine Senkung der Reallöhne behoben werden, während im Falle der zurückgestauten Inflation eine Reallohnerhöhung ratsam erscheint. ${ }^{107}$ Es muß jedoch betont werden, daß es bei der Klas-

106 Im Modell wird zwischen über Steuern finanzierter (reiner) und durch Geldschöpfung (gemischter) Fiskalpolitik unterschieden, da Staat hier als homogene Einheit von Regierung und Zentralbank betrachtet wird. Bei gemischter Fiskalpolitik ergibt sich ein Multiplikator, der größer Eins ist, bei reiner Fiskalpolitik ein Multiplikator, der zwar positiv, jedoch kleiner Eins ist.

Vgl. Felderer/Homburg (1994), S. 332-340, Hansen (1991), S. 22-29 sowie Maussner (1985), S. 233-244. Es sei darauf hingewiesen, daß im Falle zu hoher Reallöhne 
sifikation der Arbeitslosigkeit nicht um ein „entweder - oder“ geht, wie entschiedene Vertreter der neoklassischen oder keynesianischen Richtung glauben machen wollen. Häufig sind sowohl Auftragslage als auch Faktorkosten für die Arbeitslosigkeit verantwortlich, so daß beide Arten (niveaubedingter) Arbeitslosigkeit gleichzeitig auftreten können. Zudem sind Unterschiede in den einzelnen Sektoren der Volkswirtschaft festzustellen.

Nach Horn und Möller ${ }^{108}$ läßt sich der Konjunkturverlauf 1970 bis 1982 grob in drei Phasen gliedern: Ein Regime zurückgestauter Inflation zu Beginn der 70er Jahre wird (nach einer kurzen Phase weitestgehend ausgeglichener Märkte) von einer Periode klassischer Unterbeschäftigung abgelöst. Diese wiederum geht ab Mitte der 70er Jahre in eine Phase keynesianischer Unterbeschäftigung über.

Die Überschußnachfrage auf den Märkten sowie die relativ unelastischen Reaktionen der Unternehmen auf Lohnsteigerungen und Änderungen der Realzinsen zu Beginn der 70er Jahre weisen auf das Regime der zurückgestauten Inflation hin. Die Produktion wird bis etwa Mitte 1972 durch das knappe Arbeitsangebot restringiert. Die infolge der Knappheiten steigenden Reallöhne und -zinsen, gepaart mit einer fallenden Güternachfrage, führen jedoch zu einer Entspannung der Märkte. Die Ölkrise im Herbst 1973 trifft die Bundesrepublik am Beginn eines Booms, der getragen wird von einer wachsenden Exportnachfrage sowie einem in den Vorjahren entstandenen Nachholbedarf an Investitionen. Da die Produktion nicht mehr durch Engpässe am Arbeitsmarkt bestimmt wird, sind die Märkte im großen und ganzen ausgeglichen.

Die Auftragslage ist ungeachtet des Ölpreisschocks bis Ende 1974 noch zufriedenstellend. Sie kann aber - trotz gewachsener Elastizität der Beschäftigung in bezug auf die Auftragseingänge - den Beschäftigungseinbruch nicht

auch keynesianische Arbeitslosigkeit vorliegen kann. Dies ist um so wahrscheinlicher, je höher das Niveau von Löhnen und Preisen ist. Auch bei keynesianischer Unterbeschäftigung kann daher eine Reallohnsenkung empfehlenswert sein, vgl. Felderer/Homburg (1994), S. 339 f.

108 Die Untersuchung von Horn/Möller (1985) basiert auf einem Ansatz mit zeitvariablen Koeffizienten auf der Grundlage von Kalman-Filterung. Zur Methodik vgl. auch Hujer/Hansen/Klein (1989). 
verhindern, denn die Kostenfaktoren haben in zunehmendem Maße an Gewicht gewonnen, was sich in einer elastischeren Reaktion der Unternehmen auf Reallohn- und -zinsänderungen äußert. Zwei Drittel der Trendabweichung der Beschäftigungsquote des Jahres 1975 werden durch Reallohnsteigerungen erklärt, lediglich ein Viertel dagegen durch einen Rückgang der Auftragseingänge. Bei den Investitionen sind es Reallöhne und -zinsen, die den überwiegenden Teil der Trendabweichung erklären. Die Symptome der Rezession 1974/75 sind charakteristisch für das Regime klassischer Arbeitslosigkeit. In der Spätphase des Abschwungs gewinnt jedoch durch den $\mathrm{Zu}$ sammenbruch am Gütermarkt der Anteil keynesianischer Unterbeschäftigung an Gewicht. Während sich aber der Gütermarkt rasch erholt, nimmt der Einfluß der Reallöhne nach 1975 nur langsam ab. Hohe Reallöhne wirken sich auch weiterhin negativ auf Beschäftigung und Investitionen sowie Export aus. Ein direkter Vergleich des Wachstums der realen Lohnstückkosten mit der Entwicklung der Beschäftigung scheint dies zu bestätigen. Sowohl für die Gesamtwirtschaft als auch für einzelne Sektoren wie verarbeitendes Gewerbe und Dienstleistungen läßt sich ein negativer Zusammenhang feststellen. Es gibt jedoch auch Gegenbeispiele wie den Energiebereich. ${ }^{109}$ Aufgrund gestiegener Masseneinkommen ist allerdings ein positiver Effekt der Reallöhne auf den Konsum zu verzeichnen, der der Schwächung der Konsumneigung durch die hohen Zinsen entgegenwirkt.

Ende der 70er Jahre ist - aufgrund sinkender Reallohnzuwächse - kein negativer Lohneinfluß auf die Beschäftigung mehr festzustellen. Hingegen wird die Beschäftigung durch eine Periode niedriger Zinsen 1978/79 begünstigt. Die Beschäftigungslage 1979/80 kann, gemessen am Trend, als sehr positiv bezeichnet werden. Die Beschäftigungsquote (beschäftigte Personen bezogen auf das Erwerbspersonenpotential) liegt etwa 2\% über dem Trend. Etwa je ein Drittel dieser Entwicklung läßt sich auf hohe Auftragseingänge sowie die niedrigen Zinsen zurückführen. 1981/82 erfolgt jedoch ein neuerlicher Beschäftigungseinbruch. Als Ursache lassen sich vor allem hohe Realzinsen in Verbindung mit einer seit Mitte der 70er Jahre nahezu kontinuierlich gewachsenen Realzinselastizität der Beschäftigung anführen. Ein Wiederan-

109 Vgl. Franz/König (1986). Ihrer Meinung nach ist es jedoch wichtig, zwischen den Arbeitsstunden und der Beschäftigung gemessen an der Anzahl der Arbeitsplätze zu unterscheiden. Im Rahmen ihrer Untersuchung berücksichtigen sie daher auch Überstundenprämien und den Auslastungsgrad der Arbeit. 
stieg der Beschäftigung, nachdem der negative Zinseffekt seit Ende 1981 nachließ, wurde jedoch durch einen Rückgang der Aufträge in der Folge des zweiten Ölpreisschocks verhindert, der noch einschneidender als 1975 war. Eine ähnliche Entwicklung zeigt sich bei den Investitionen. Der Gütermarkt wird in den Jahren 1981/82 von der Entwicklung auf dem Arbeitsmarkt dominiert. Die gestiegene Massenarbeitslosigkeit schlägt sich erstmals in sinkendem Konsum und Bruttosozialprodukt nieder. Die Parameter weisen somit auf einen Zustand keynesianischer Arbeitslosigkeit hin. Gleichzeitig deutet die Zinsreagibilität von Beschäftigung und Investitionen auf einen Kapitalmangel hin. Dieser dürfte auf die zweite Hälfte der 70er Jahre zurückzuführen sein, in der sich die Lohnentwicklung negativ auf das Investitionsverhalten ausgewirkt hatte.

Die Ergebnisse von Horn und Möller werden von verschiedenen jüngeren Untersuchungen im wesentlichen bestätigt. Untermauert wird die vorgenommene Regimeeinteilung auch dadurch, daß die verschiedenen Autoren trotz z.T. unterschiedlicher Analysemethoden zu ähnlichen Ergebnissen gelangen. Coen und Hickman ${ }^{110}$ kommen (auf der Basis eines Modells von Hickman) ebenfalls zu dem Ergebnis, daß die Löhne im Laufe der 70er Jahre einen zunehmenden Einfluß gewinnen, der in ihrer Berechnung nach 1976 tendenziell wieder nachläßt. Ab Mitte der 70er Jahre gewinnt jedoch die keynesianische Komponente an Gewicht. Die Arbeitslosigkeit zu Beginn der 80er Jahre ist eindeutig keynesianisch geprägt. 1973 entfallen 7\% der Arbeitslosigkeit auf die Nachfrage-, $37 \%$ auf die Lohnkomponente. In den folgenden Jahren kehrt sich das Verhältnis um. Im Durchschnitt der Jahre 1974 bis 1979 werden $47 \%, 1980$ bis 1983 sogar $76 \%$ durch die Nachfrage erklärt. Der Anteil der Lohnkomponente sinkt hingegen in den entsprechenden Jahren auf 28 bzw. 6\%. Die Prozentsätze beziehen sich dabei auf eine um versteckte Arbeitslosigkeit korrigierte Arbeitslosenquote, wobei der Anteil der versteckten Arbeitslosigkeit insgesamt gestiegen ist. ${ }^{111}$ Es sei betont, daß es hier letztlich nicht um eine exakte Bezifferung der Regimeanteile geht, die nicht zuletzt aufgrund der Abhängigkeit der Ergebnisse von der jeweiligen Modellspezifikation - auch kaum möglich sein dürfte, sondern um das Auf-

110 Vgl. Coen/Hickman $(1987,1988)$.

111 Die Differenz der Summen beider Anteile zu 100\% entspricht dem geschätzten Anteil „natürlicher" Arbeitslosigkeit, vgl. Coen/Hickman (1988), insbesondere S. 190192. 
zeigen von zu beobachtenden Tendenzen. Entorf et al. ${ }^{112}$, Drèze und Bean ${ }^{113}$ sowie Hansen ${ }^{114}$ stellen auf der Basis eines Modellansatzes von Sneessens und Drèze ${ }^{115}$ ebenfalls eine Dominanz des keynesianischen Regimes 1975 sowie zu Beginn der 80 er Jahre fest. Die Analyse von Laroque ${ }^{116}$, die auf einer Modellschätzung mit Hilfe der Pseudo-Maximum-Likelihood-Methode beruht, bestätigt dies ebenfalls. Die Untersuchungen von Entorf et al., Laroque sowie Hansen stützen außerdem die Klassifikation der Arbeitslosigkeit zu Beginn der 70er Jahre als zurückgestaute Inflation sowie den relativ hohen Anteil klassischer Unterbeschäftigung bis 1975. Auch der nach der Rezession 1975 fortbestehende Einfluß der Reallöhne bis Ende der 70er Jahre kann durch ihre Ergebnisse prinzipiell bestätigt werden. ${ }^{117}$ Bemerkenswert ist auch, daß die genannten Resultate mit Untersuchungen auf der Basis von Unternehmensbefragungen relativ gut übereinstimmen. ${ }^{18}$

112 Vgl. Entorf/Franz/König/Smolny (1989).

113 Vgl. Drèze/Bean (1990).

114 Vgl. Hansen (1991). Vgl. hierzu auch Hujer/Hansen/Klein (1994), S. 191-193.

115 Vgl. Sneessens/Drèze (1986).

116 Vgl. Laroque (1989).

117 Unterschiede zeigen sich bei den einzelnen Untersuchungen eher für den Zeitraum nach 1982/83. Während sich bei Drèze/Bean (1990) und Laroque (1989), obgleich deren Betrachtungen lediglich bis 1984 bzw. 1985 gehen, eine fortgesetzte Dominanz des keynesianischen Regimes andeutet, gewinnt bei Entorf/Franz/König/ Smolny (1989) sowie Hansen (1991) das klassische Regime wieder an Bedeutung, wobei sich dieses mit der geringen Investitionsquote dieser Jahre erklären läßt, vgl. Hujer/Hansen/Klein (1994).

Vgl. Möller (1982), S. 159-167 für einen Zeitraum von 1966 bis 1978, König/Entorf (1990) für die 80er Jahre und König/Zimmermann (1986) für den Beginn der 80er Jahre. 


\subsection{Ein erweiterter Erklärungsansatz zur Entwicklung der Arbeitslosigkeit}

$\mathrm{Zu}$ hohe Löhne oder mangelnde Nachfrage können jedoch die seit Mitte der 70er Jahre herrschende Arbeitslosigkeit nur zum Teil erklären. Im folgenden soll daher in Anlehnung an Layard et al. ${ }^{119}$ die Erklärung der seit 1974 herrschenden Arbeitslosigkeit auf eine breitere Basis gestellt werden. Ausgangspunkt ist - in Fortfuihrung zu Untersuchungen zur Phillips-Kurve - der offensichtliche Zusammenhang zwischen der (trendbereinigten) Arbeitslosenquote und der Veränderung der Inflationsrate (vgl. Schaubild 4). Letztlich läßt sich fast jede Arbeitslosigkeit durch eine expansive Nachfrage beseitigen. Einer fortgesetzt expansiven Fiskal- oder Geldpolitik steht aber - abgesehen von einer explodierenden Staatsverschuldung - eine akzelerierende Inflation gegenüber. Eine niedrige Arbeitslosenquote wird nach den bisherigen Erfahrungen oft mit höheren Inflationsraten erkauft. ${ }^{120}$ Umgekehrt führt eine konsequente Bekämpfung der Inflation häufig zu einem Anstieg der Arbeitslosenquote. Eine Betrachtung der Arbeitslosigkeit muß daher stets auch die Preisentwicklung mit einbeziehen. Im Mittelpunkt der weiteren Überlegungen steht daher die sog. NAIRU (Non-Accelerating Inflation Rate of Unemployment), also diejenige Arbeitslosenquote, bei der die Inflationsrate stabil bleibt. Hintergrund ist der Verteilungskampf zwischen organisierten Arbeitnehmer- und Arbeitgebergruppen. Während die Arbeitnehmer eine Erhöhung der Reallöhne anstreben, richten sich die Unternehmen bei ihrer Preissetzung neben der Situation auf den Absatzmärkten nach den Lohnstückkosten. Schaukeln sich so die Ansprüche an das Sozialprodukt gegenseitig auf, dann wird ein Wachstum der Inflationsrate in Gang gesetzt (LohnPreis-Spirale). Um dies zu verhindern, ist ein Mechanismus erforderlich, der die Ansprüche der Arbeitnehmer- und Arbeitgeberseite in Einklang bringt. In der Regel wird eine Koordination über den Arbeitsmarkt erfolgen. Bei erhöhter Arbeitslosigkeit werden sich die Gewerkschaften eher mit Lohnforderungen zurückhalten, während umgekehrt die Absatzlage der Unternehmen von der Arbeitsmarktsituation beeinflußt wird. Die NAIRU ist diejenige Arbeitslosenquote, die einen Ausgleich der Ansprüche herstellt; der Arbeits-

119 Vgl. Layard/Nickell/Jackman (1994). Zu den folgenden Ausführungen vgl. ebenfalls

120 Franz (1994), insbesondere Kapitel 9.

Eine gewisse Ausnahme bilden in der Bundesrepublik die 60er Jahre, in denen ein Rückgriff auf ausländische Arbeitsmärkte möglich war, vgl. Franz (1994), S. 346. 
markt wird also nicht notwendigerweise geräumt, befindet sich jedoch in einem Gleichgewicht. Gleichzeitig gibt die NAIRU an, wie weit die Arbeitslosenquote reduziert werden kann, ohne daß ein Anstieg der Inflationsrate induziert wird.

Damit wird auch deutlich, daß sich das Problem der Arbeitslosigkeit nicht durch ein einfaches Mehr an Inflation lösen läßt ${ }^{121}$, denn eine Reduzierung der Arbeitslosenquote ist nur dann möglich, wenn die Inflationsrate ständig beschleunigt wird. Die gleichgewichtige Arbeitslosenquote ist um so niedriger, je flexibler die Reallöhne reagieren. Als eine Erklärung für das Beharrungsvermögen der Arbeitslosigkeit sind daher in der Diskussion Lohn- und Preisrigiditäten angeführt worden, die theoretisch mit unvollständigen Informationen (Job-Search-Modelle, Theorie der impliziten Kontrakte), mit Transaktionskosten (Effizienzlohntheorien) oder gewerkschaftlichem Verhalten (Insider/Outsider-Theorie) begründet werden. Hierauf soll jedoch nicht näher eingegangen werden. ${ }^{122}$ Hier vollzieht sich der Übergang von der makroökonomischen Beschäftigungstheorie zur mikroökonomischen Arbeitsmarkttheorie.

Langfristig ist die Arbeitslosenquote damit allein durch Angebotsfaktoren festgelegt und entspricht der NAIRU. Kurzfristig wird die Beschäftigung dagegen durch das Zusammenspiel von aggregierter Nachfrage und kurzfristigem aggregierten Angebot bestimmt. Angebotsschocks in Form der beiden Ölkrisen haben zu einem Anstieg der NAIRU geführt. ${ }^{123}$ Dies läßt sich wie folgt erklären: Bei einem preiserhöhenden Angebotsschock kann die Inflationsrate nur durch eine entsprechende relative Lohnsenkung oder Reduzierung der Unternehmensgewinne stabilisiert werden. Da ein größerer Teil des Sozialprodukts ins Ausland fließt, engt sich der Verteilungsspielraum im Inland ein. Bleibt eine Anpassung der Lohnforderungen aus und können (oder wollen) die Firmen ihren Gewinnzuschlag nicht reduzieren, re-

121 Eine Wahl zwischen Inflation und Arbeitslosigkeit, wie sie in der Parole Schmidts „Lieber 5\% Inflation als 5\% Arbeitslosigkeit“ im Wahlkampf 1972 zum Ausdruck kommt, besteht gerade nicht.

Einen knappen Überblick gibt beispielsweise Berthold (1988). Einen Überblick zur empirischen Relevanz der einzelnen Theorien liefern z.B. Franz (1994) oder Layard/ Nickell/Jackman (1994).

Vgl. hierzu Franz (1985). 


\section{Schaubild 4}

\section{Arbeitslosigkeit und Inflation}

- früheres Bundesgebiet -

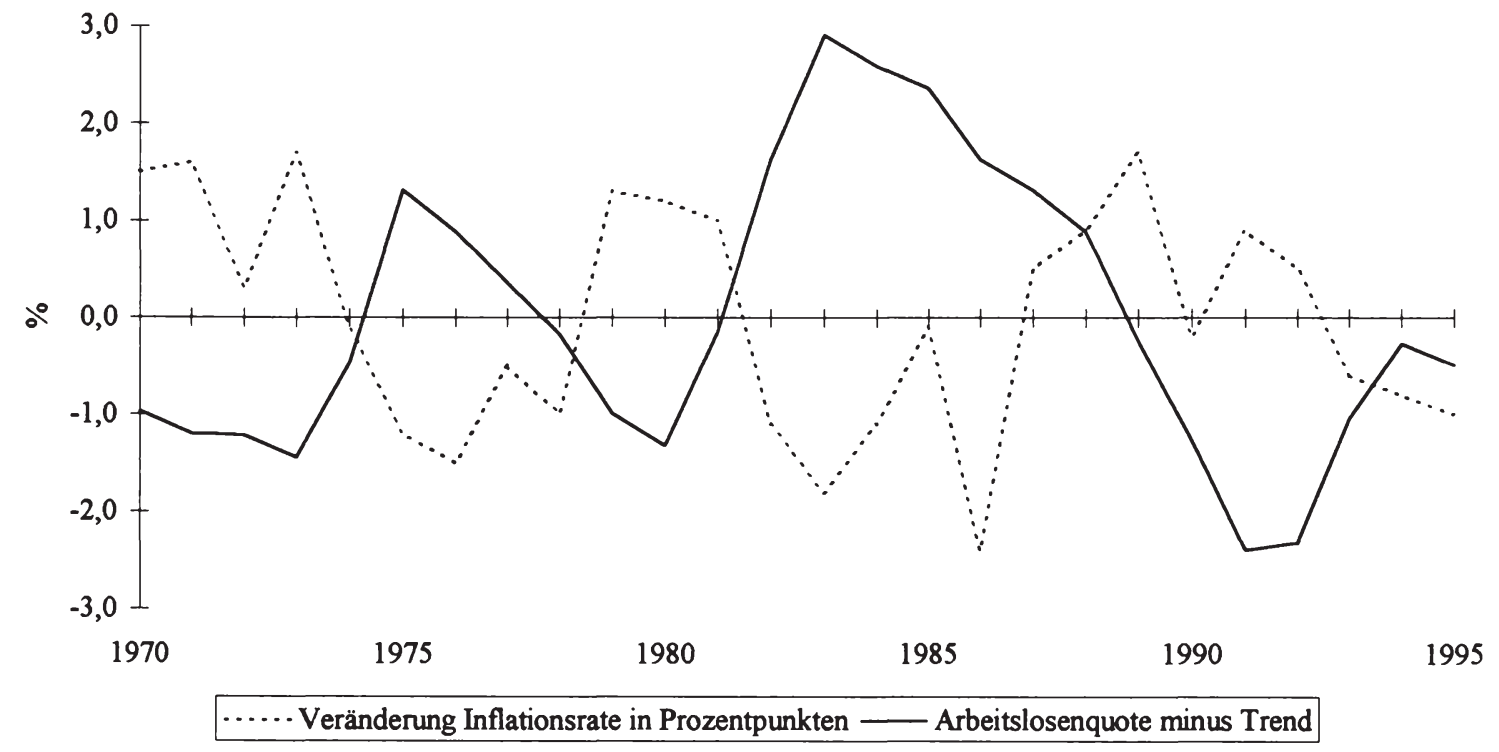

Anmerkung: Inflationsrate auf Basis der Verbraucherpreise.

Arbeislosenquote minus Trend

Quelle: Sachverstăndigenrat zur Begutachtung der gesamtwirtschaftlichen Entwicklung $(1995,1996)$; eigene Berechnungen. 
sultiert dies in einer höheren Arbeitslosigkeit. Es sind jedoch einige Anmerkungen notwendig. Primär wächst die Arbeitslosigkeit infolge eines Angebotsschocks zunächst, weil durch die höheren Preise die Nachfrage reduziert wird. Wird nun versucht, durch eine restriktive Politik dem Anstieg der Inflationsrate entgegenzuwirken, dann ergibt sich der soeben beschriebene zusätzliche Anstieg der Arbeitslosigkeit. Bezogen auf den Anstieg der Inflationsrate - nicht jedoch auf die Inflationsrate selbst - hat der Angebotsschock allerdings lediglich transitorischen Charakter. Nachdem sich die Preise auf einem neuen höheren Niveau eingependelt haben, geht die NAIRU auf ihr Niveau vor dem Schock zurück. ${ }^{124 / 125}$

Weder adverse Schocks noch die Lohnentwicklung können jedoch erklären, warum sich die Arbeitslosigkeit in den wirtschaftlichen Aufschwungphasen nur sehr langsam von ihrem jeweils krisenbedingten Anstieg erholte. Seit einiger Zeit wird daher mit dem der Physik entlehnten Phänomen der Hysteresis argumentiert. In den Wirtschaftswissenschaften wird damit die Pfadabhängigkeit dynamischer ökonomischer Systeme beschrieben. Der Gleichgewichtswert eines Systems hängt danach davon ab, auf welchem Wege dieses Gleichgewicht erreicht wurde. Bezogen auf die Arbeitslosigkeit bedeutet dies, daß die Arbeitslosenquote von ihrer früheren Entwicklung mitbestimmt wird, vergangene hohe Arbeitslosigkeit sich also negativ auf die jeweils aktuelle Beschäftigungssituation auswirkt. Steigt die Arbeitslosenquote beispielsweise infolge eines Ölpreisschocks, dann kehrt sie, selbst wenn der Angebotsschock in vollem Umfang rückgängig gemacht wird, nicht wieder auf ihr ursprüngliches Niveau zurück. Franz hat für die Bundesrepublik einige Evidenz dafür gefunden, daß ein rezessionsbedingter Anstieg der Arbeitslosigkeit mit einer höheren zukünftigen friktionellen oder strukturellen Arbeitslosigkeit einhergeht. ${ }^{126}$ Hysteresis führt somit zu steigenden Werten der NAIRU.

Grund für ein solches persistentes Verhalten der Arbeitslosigkeit kann bei-

124

125

126

Vgl. Franz (1994), S. 349 f.

Wie sich zeigte, ist ein negativer Angebotsschock sowohl mit einer beschleunigten Inflationsrate als auch mit einem Anstieg der Arbeitslosigkeit verbunden. Im Falle eines negativen Nachfrageschocks geht hingegen die wachsende Arbeitslosigkeit mit einer sinkenden Inflationsrate einher.

Vgl. Franz (1987). 
spielsweise sein, daß entmutigte Arbeitslose die Suche nach einem Arbeitsplatz aufgeben bzw. von den Unternehmen als ungeeignet abgelehnt werden. Es zeigt sich zudem, daß die Entwicklung der Arbeitslosigkeit durch einen wachsenden Anteil Langzeitarbeitsloser bestimmt wird. Nach den Rezessionen 1975 und 1982/83 ist jeweils ein Verharren auf einem höheren Niveau zu verzeichnen, wobei der Anstieg Anfang der 80er Jahre ausgeprägter war. ${ }^{127}$ Als Begründung wird die mit langfristiger Arbeitslosigkeit verbundene Entwertung des Humankapitals der Erwerbslosen angeführt. Licht und Steiner ${ }^{128}$ finden hierfür (allerdings auf Basis von Schätzungen für 1984 bis 1987) einige Evidenz. ${ }^{129}$

Abschließend soll auf eine mögliche Erhöhung der NAIRU durch sog. Mismatch-Arbeitslosigkeit hingewiesen werden. Mismatch hat seine Ursache in einer nicht übereinstimmenden Struktur von Arbeitsangebot und -nachfrage. ${ }^{130}$ Ein Vergleich der Arbeitslosenquote mit dem Anteil offener Stellen zeigt $^{131}$, daß die gleiche Anzahl offener Stellen früher mit einer geringeren Arbeitslosigkeit verbunden war (jeweils bezogen auf die Erwerbspersonen). ${ }^{132}$ In der Regel werden drei Gründe für das Zustandekommen eines Mismatch genannt: (a) ein Informationsdefizit des Arbeitsplatzsuchers bzw. des Unternehmens bezüglich vorhandener Vakanzen resp. geeigneter Bewerber, (b) qualitative Differenzen zwischen Sucher und angebotener Stelle, (c) Sucher und Unternehmung sind in unterschiedlichen Regionen ansässig und immobil. Während der regionale Mismatch in den Jahren 1976 bis 1979 einen leichten Anstieg verzeichnet und dann etwa auf diesem Niveau verharrt, ist ein deutlicher qualifikationsbedingter Mismatch festzustellen,

127 Vgl. Grassinger (1993), S. 82.

128 Licht/Steiner (1991).

129 Dabei ist zunächst unerheblich, ob ein Humankapitalverlust tatsächlich erfolgt oder ob er von den Unternehmen mangels anderer Entscheidungskriterien bei der Einstellung lediglich vermutet wird. Zum Humankapitalansatz vgl. ausführlich Grassinger (1993). Dort wird auch diskutiert, inwieweit die empirischen Ergebnisse nicht möglicherweise auch mit anderen Ansätzen erklärt werden können.

130 Zur näheren Begründung vgl. z.B. Franz (1994), Kapitel 6 sowie Layard/Nickell/ Jackman (1994), Kapitel 6.

131 Der Zusammenhang zwischen Vakanzquote und Arbeitslosenquote wird in der Regel durch eine sog. Beveridge-Kurve abgebildet.

132 Die Beveridge-Kurve verzeichnet jeweils nach 1973 und 1983 eine Verschiebung, vgl. Franz (1994), S. 200 oder Layard/Nickell/Jackman (1994), S. 36 f. 
wobei 1979 ein Rückgang stattfindet, dem jedoch ein abrupter Anstieg bis 1983 folgt. Wenn es also strukturelle Ursachen für die hohe Arbeitslosigkeit gibt, dann sind sie insbesondere mit berufsspezifischen Faktoren zu erklären. ${ }^{133}$ Derartige Phänomene treten nicht kurzfristig auf, sondern sind Folgen längerfristiger Veränderungsprozesse. Sie sind auch nicht kurzfristig zu beheben. Im vorliegenden Fall ist wohl insbesondere die mit Beginn der 70er Jahre erstmalig und Anfang der 80er Jahre verstärkt in alle Wirtschaftsbereiche vordringende neue mikroelektronische Technologie zu beachten. Sie verändert das Anforderungsprofil und die Produktionsprozesse; der Anpassungsprozeß ist bis heute nicht abgeschlossen.

Zusammenfassend läßt sich also feststellen, daß unterschiedliche Gründe für einen Anstieg der NAIRU verantwortlich sind. ${ }^{134}$ Dabei wurde deutlich, daß die Begründungen für den Anstieg in den 70er Jahren sowohl auf der Makro- als auch auf der Mikroebene zu suchen sind. Die im vorangegangenen Abschnitt beschriebene Erklärung der Arbeitslosigkeit auf der Basis der Rationierungsmodelle steht dabei keineswegs im Gegensatz zu dem hier vorgestellten breiter angelegten Erklärungsansatz. Sie beschreiben vielmehr die Situation des Arbeitsmarktes aus kurzfristiger Sicht, während die NAIRU die langfristige Entwicklung widerspiegelt, jedoch auch, wie gezeigt wurde, auf kurzfristige Schwankungen an den Güter- und Arbeitsmärkten reagiert.

133 Vgl. Franz (1994), S. 213-219. Eine Erhöhung des generellen Mismatchniveaus seit Beginn der 70er Jahre wird auch von Entorf bestätigt, vgl. Entorf (1995).

Empirische Schätzungen der NAIRU für die Bundesrepublik fallen bei den einzelnen Autoren sehr unterschiedlich aus; die Differenzen gehen z.T. über $2 \%$ hinaus. Einen Überblick gibt Franz (1994), S. 357. 


\subsection{Die Beschäftigungspolitik vor dem Hintergrund der Erklärungsansätze}

Mit Hilfe des vorstehenden Gerüstes soll nun versucht werden, die beschäftigungspolitischen Maßnahmen zu beurteilen - immer unter der Prämisse, daß Phänomene wie Hysteresis zur damaligen Zeit noch nicht bekannt waren und daher negative Erfahrungen in der Beschäftigungspolitik quasi unumgänglich und nicht angemessen zu beurteilen waren. Die obigen Ausführungen haben deutlich gemacht, daß das einer Therapieempfehlung zugrundeliegende Diagnoseproblem nur empirisch lösbar ist. ${ }^{135}$ Insbesondere hat sich gezeigt, daß eine solche Diagnose selbst im Rückblick, mit dem Wissen um die Wirksamkeit oder Nicht-Wirksamkeit der getroffenen Maßnahmen, nicht immer eindeutig ausfällt. Daher geht es vor allem darum, mögliche Gründe aufzuzeigen, warum die Beschäftigungsprogramme trotz ihres zum Teil enormen Volumens insgesamt nur mäßige Wirkungen zeitigten. Dabei ist zu beachten, daß in den oben beschriebenen ökonometrischen Schätzungen die Wirkungen staatlicher Maßnahmen bereits mit erfaßt sind. Der Begriff der Beschäftigungspolitik wird im folgenden sehr weit ausgelegt und soll alle Maßnahmen erfassen, die - direkt oder indirekt - darauf abzielen, einen hohen Beschäftigungsstand zu erhalten bzw. wiederherzustellen.

Beschäftigungspolitik in diesem Sinne wurde erstmals Ende 1973 betrieben. ${ }^{136}$ Angesichts der am 16. Oktober ausgebrochenen Ölkrise lockerte die Bundesregierung ihr Restriktionsprogramm vom Februar und Mai des gleichen Jahres. Die Investitionsteuer wurde aufgehoben, die degressive Abschreibung für bestimmte Wirtschaftsgüter sowie die Sonderabschreibung für Wohngebäude wurde wieder zugelassen und die bis dahin gestoppten bzw. gedrosselten Kreditprogramme des ERP-Sondervermögens wurden wieder aufgenommen. Zuvor war bereits beschlossen worden, die für 1974 vorgesehene Streckung von Mitteln im Rahmen der Gemeinschaftsaufgaben nicht vorzunehmen. Der Verhinderung von Beschäftigungseinbrüchen sollte auch das „Sonderprogramm für Gebiete mit speziellen Strukturproblemen“

135 Vgl. Hansen (1991), S. 4.

$136 \mathrm{Zu}$ den einzelnen Konjunktur- und Beschäftigungsprogrammen vgl. die Finanzberichte des Bundesministeriums der Finanzen, die Jahresgutachten des Sachverständigenrates und die Geschäftsberichte der Deutschen Bundesbank für die Jahre 1973 bis 1979 . 
vom Februar 1974 dienen, das einschließlich der Beteiligungen der Länder Ausgaben in Höhe von 900 Mio. DM umfaßte. Im März wurde die für 1974 vorgesehene „Schuldendeckel“-Verordnung zurückgezogen und ein Investitionsprogramm aufgelegt, das für den Fall eines konjunkturellen $\mathrm{Ab}$ schwungs zusätzliche Mittel für Investitionen in Höhe von $5 \mathrm{Mrd}$. DM vorsah. Ein Anstieg der Arbeitslosigkeit im Laufe des Jahres 1974 wurde jedoch nicht verhindert. Die Bundesregierung betrachtete ihre Politik dennoch als konzeptionell erfolgreich und kündigte eine Fortführung der Stabilisierungspolitik an. Im September beschloß sie ein „Sonderprogramm zur regionalen und lokalen Abstützung der Beschäftigung", das Baumaßnahmen in Höhe von 700 Mio. DM in Orten und Gebieten mit überdurchschnittlicher Arbeitslosigkeit vorsah (finanziert durch Bund und Länder) sowie Investitionsmaßnahmen des Bundes in Höhe von 250 Mio. DM. Zu einem großen Teil ist dieses Programm aus dem bei der Bundesbank stillgelegten Investitionsteueraufkommen (Konjunkturausgleichsrücklage) finanziert worden. Darüber hinaus wurde im Dezember ein „Programm zur Förderung von Beschäftigung und Wachstum bei Stabilität" mit einem Gesamtvolumen von 1,73 Mrd. DM aufgelegt. Es beinhaltete zusätzliche Ausgaben zur Stärkung der Investitionen, für bestimmte Gebiete Lohnkostenzuschüsse an Arbeitgeber, Mobilitätszulagen an arbeitslose Arbeitnehmer und Investitionszuschüsse im Bereich des sozialen Wohnungsbaus. Finanziert wurde das Programm vornehmlich aus Mitteln aus dem Stabilitätszuschlag. Darüber hinaus wurde für Anlageinvestitionen eine zeitlich befristete Investitionszulage von 7,5\% gewährt, die Höchstdauer des Bezugs von Kurzarbeitergeld verlängert, ein Vorziehen der für 1975 geplanten Investitionen möglichst ins 1. Halbjahr und eine vollständige Freigabe von Verpflichtungsermächtigungen für Investitionen vorgesehen. Zudem wurden über das ERP-Sondervermögen sowie die Kreditanstalt für Wiederaufbau Finanzierungshilfen in Höhe von 0,5 bzw. mindestens 1 Mrd. DM bereitgestellt. Im August 1975 wird von der Bundesregierung zur Stützung der Nachfrage ein „Programm zur Stärkung von Bau- und anderen Investitionen" beschlossen, das mit einem Volumen von 5,75 Mrd. DM die vorgenannten Konjunkturprogramme größenordnungsmäßig weit übertrifft. 3,15 Mrd. werden dabei vom Bund finanziert. Das Programm umfaßt schwerpunktmäßig die Teile „Kommunale Infrastruktur und Stadtsanierung", „Bundeseigene Investitionen“, daneben stellt es aber auch Mittel zur Verfügung zur Wohnungsmodernisierung, für Arbeitsbeschaffungsmaßnahmen, zur Zwischenfinanzierung von Bausparverträgen 
sowie für Darlehen zur Erleichterung der Finanzierung kommunaler Investitionen und zur Verstärkung der ERP-Umweltschutzprogramme. Zusätzlich expansiv ausgerichtet waren die Gesetze zur Reform der Vermögensteuer und der Erbschaftsteuer, die rückwirkend zum 1. Januar 1974 in Kraft traten, sowie die Reform der Gewerbesteuer und die Steuer- und Kindergeldreform, die jeweils zum 1. Januar 1975 in Kraft traten. Der Sachverständigenrat errechnet für den öffentlichen Haushalt in der Abgrenzung der Volkswirtschaftlichen Gesamtrechnung für 1974 und 1975 einen fiskalischen Impuls von 14,6 bzw. 35,9 Mrd. DM, im Vergleich zu 7,3 Mrd. 1973. ${ }^{137}$ Darüber hinaus wurde im Januar 1976 ein arbeitsmarkt- und berufspolitisches Programm zur Bekämpfung der Jugendarbeitslosigkeit und zur Förderung schwer vermittelbarer Arbeitsloser im Umfang von 300 Mio. DM beschlossen sowie im November in einem weiteren arbeitsmarktpolitischen Programm 430 Mio. DM zur Erhöhung der Mobilität der Arbeitslosen und zur Erleichterung ihrer beruflichen Eingliederung zur Verfügung gestellt. Trotz dieser umfangreichen Maßnahmen kam es 1975 zum Einbruch am Arbeitsmarkt. 1976 stieg das Bruttosozialprodukt real wieder um 5,5\%, brachte aber keine nennenswerte Erholung des Arbeitsmarktes mit sich. Hatte man bis dahin - trotz der bereits zu Beginn der 70er Jahre ernüchternden Erfahrungen mit der kontraktiv orientierten Stabilisierungspolitik - noch geglaubt, Vollbeschäftigung sichern zu können ${ }^{138}$, muß nun konstatiert werden, daß auch im Falle expansiver Maßnahmen die traditionelle Konzeption erfolglos bleibt oder doch zumindest der „Erfolg“ bestenfalls darin besteht, daß die Situation nicht noch schlechter ausfällt.

Wieso hat sich nun die Fiskalpolitik als unwirksam erwiesen? Anhand verschiedener ökonometrischer Studien wurde gezeigt, daß sich die Situation 1975 durchaus als ein Fall keynesianischer Unterbeschäftigung charakterisieren läßt und so der typische Fall für den Einsatz der Fiskalpolitik war. Sicher wäre es falsch, den expansiven Maßnahmen jegliche Wirkung abzusprechen. Bevor sie jedoch wirksam werden konnten, setzte sich bereits der negative Einfluß der Reallohnentwicklung durch. Die im Zuge des Ölpreisschocks beschleunigte Inflation hätte nun möglicherweise die realen Lohnkosten reduzieren können. Dies wurde jedoch durch eine restriktive Geldpo-

137 Vgl. Sachverständigenrat zur Begutachtung der gesamtwirtschaftlichen Entwicklung (1979), S. $111 \mathrm{ff}$., siehe auch Fußnote 143.

138 Die Bundesregierung hatte sogar eine Vollbeschäftigungsgarantie gegeben. 
litik verhindert. Der durch den Angebotsschock ausgelöste vorübergehende Anstieg der Arbeitslosigkeit wurde also durch den Versuch, die Inflation zu stabilisieren, zusätzlich - und zwar dauerhaft - erhöht. Dabei wurde der scharfe Restriktionskurs der Bundesbank in der Öffentlichkeit zunächst kaum wahrgenommen. Im Gegenteil gibt die Ölkrise im Herbst 1973 Inflationserwartungen neuen Auftrieb und führt Anfang 1974 (es herrscht noch Arbeitskräfteknappheit) zu Tarifabschlüssen, die Nominallohnsteigerungen von ca. $13 \%$ vereinbaren, die die Unternehmen wiederum in die Preise zu überwälzen hoffen. Die Verantwortung für den damit ausgelösten Anstieg der Arbeitslosigkeit weist die Bundesbank jedoch klar von sich - und den Gewerkschaften zu. ${ }^{139}$

Zudem zeichnen sich in der Bundesrepublik strukturelle Veränderungen ab; primärer und sekundärer Sektor verlieren, der tertiäre Sektor gewinnt an Bedeutung. Der Anteil des sekundären Sektors am Bruttoinlandsprodukt war bis dahin im Vergleich zum Durchschnitt der OECD-Länder relativ hoch. Die Ursachen für seine relative Stärke lagen zum einen in einem starken Bevölkerungswachstums zum anderen in einem hohen Nachholbedarf. Verstärkt wurde diese Entwicklung durch eine wirtschaftspolitische Konzeption, die auf einen Investitions- und Wachstumskurs setzte. Zudem war die Exportstruktur gut auf die international wachsenden Märkte zugeschnitten und die Wettbewerbsposition der Bundesrepublik aufgrund einer Unterbewertung der DM äußerst günstig. Mitte der 70er Jahre zeichnete sich jedoch eine Veränderung der Rahmenbedingungen $a b$. Veränderungen im Bedarf, Höherbewertung der DM sowie eine nachlassende Wachstumsdynamik im

139 Nach Ansicht der Bundesbank seien die Gewerkschaften frei gewesen „weniger hohe Lohnsteigerungen zu vereinbaren und damit den Druck auf die Gewinnmargen und seine negative Wirkung auf Investitionen und Beschäftigung (...) zu vermindern. Die negativen Konsequenzen der getroffenen lohnpolitischen Entscheidungen können also nicht Bundesregierung und Bundesbank angelastet werden, die deutlich erklärt hatten, daß sie 1974 die Stabilitätspolitik fortsetzen würden und überdies auch zum Ausdruck brachten, daß lohnpolitische Entscheidungen, die mit dieser Grundlinie nicht übereinstimmten, das Arbeitsplatzrisiko erhöhen würden“, Deutsche Bundesbank (1974), S. 17. Die Beschäftigung wurde praktisch erst dann durch die Inflation bedroht, als die Bundesbank sie wirksam bekämpfen konnte, d.h. nachdem das System von Bretton Woods zusammengebrochen war und die Bundesbank ungehindert von außenwirtschaftlichen Einflüssen ihre restriktive Politik durchsetzen konnte, vgl. Spahn (1988), S. 55 und 71-74. 
Ausland lösten einen Strukturwandel aus. ${ }^{140}$ Diese schufen zusätzlichen Anpassungsbedarf auf dem Arbeitsmarkt. Mitte 1978 wurden im Rahmen eines Nachtragshaushalts (ausgabenneutral) Mittel in Höhe von 0,94 Mrd. DM für zusätzliche Investitionshilfen, hauptsächlich zur Förderung des Montanbereichs, bereitgestellt.

Nach den negativen Erfahrungen mit der expansiven Fiskalpolitik in der Rezession 1975 und der Einsicht, daß das Beschäftigungsproblem nur mittelfristig zu lösen sei, löste sich die Bundesregierung, wie in Abschnitt 1.2 ausführlich beschrieben wurde, sukzessive von der Nachfragepolitik und wendete sich einer Angebotspolitik zu. Zudem gewann, bedingt durch den massiven Rückgang der realen Investitionsquote in der ersten Hälfte der 70er Jahre, die Investitionsförderung insgesamt an Gewicht, da mangelnde Investitionen sich negativ auf das Produktionspotential auswirkten und damit auf die längerfristigen Wachstumschancen. Zudem wurden sie als eine wesentliche Ursache für die Arbeitslosigkeit angesehen. Im März 1977 beschloß die Bundesregierung ein mehrjähriges „Programm für Zukunftsinvestitionen“ (ZIP), das Investitionsausgaben der Gebietskörperschaften im Umfang von 13,8 Mrd. DM bis einschließlich 1981 vorsah, wovon 3,5 Mrd. DM an Aufträgen bereits 1977 vergeben werden sollten (vgl. Abschnitt 1.4). Vorgesehen waren öffentliche Investitionen in den Bereichen Verkehrswesen, Energieversorgung, Wasserwirtschaft sowie für eine bessere Wohnumwelt und für die Berufsbildung. Durch dieses Programm sollten auch private Investitionen angeregt werden. Zur Stützung der Bauwirtschaft und zur Bekämpfung der Arbeitslosigkeit wurde darüber hinaus im Mai eine Aufstockung und Fortsetzung des regionalen Wohnungsbauprogramms sowie die Bereitstellung zusätzlicher Mittel für Arbeitsbeschaffungsmaßnahmen beschlossen. Zur Förderung von Wachstum und Beschäftigung wurde außerdem - nach Änderungen im Vermittlungsausschuß von Bundestag und Bundesrat - im Oktober das "Gesetz zur Steuerentlastung und Investitionsförderung“ beschlossen, das neben sozialpolitischen Maßnahmen u.a. eine Verbesserung der degressiven Abschreibung vorsah.

Im Januar 1978 legte die Bundesregierung einen Gesetzentwurf vor, der zusätzliche Investitionsanreize, wie eine Verdoppelung des Zulagensatzes von

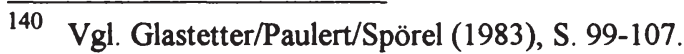


$7,5 \%$ auf $15 \%$ für begünstigte Investitionsaufwendungen sowie eine Ausweitung der Begünstigung auf Gebäude und bestimmte immaterielle Wirtschaftsgüter, die der Forschung und Entwicklung dienen, vorsah. Insgesamt blieb die Wirkung der Investitionsförderungsmaßnahmen, gemessen am Umfang, allerdings gering. Die Bundesbank führt dies darauf zurück, daß sich in den gesamtwirtschaftlichen Rahmenbedingungen tieferliegende Wandlungen vollzogen haben, wie der Rückgang der Bevölkerung, ein geringerer technischer Fortschritt, zusätzliche Kosten durch Umweltschutzauflagen, eine veränderte Einkommensverteilung zu Lasten der Unternehmer und eine Umstrukturierung der außenwirtschaftlichen Verhältnisse. ${ }^{141}$ Glastetter et al. schätzen Beschäftigungseffekte durch Investitionen generell geringer ein. Ein Vergleich von Investitionen und Erwerbstätigkeit im Betrachtungszeitraum zeigt, daß eine Verminderung der Investitionstätigkeit zwar stets mit einer verringerten Beschäftigung einhergeht, umgekehrt eine solche Gesetzmäßigkeit aber nicht festzustellen ist. Ein gewisser positiver Zusammenhang läßt sich erkennen, aber auch sehr hohe Investitionszunahmen bewirken lediglich eine geringe Zunahme der Erwerbstätigkeit. Dafür dürfte nicht zuletzt der hohe Anteil an Rationalisierungsinvestitionen ausschlaggebend sein. ${ }^{142}$ Eine weitere mögliche Begründung liegt, wie oben dargestellt wurde, in dem - trotz Investitionserleichterungen - nur langsam nachlassenden Einfluß der Kostenfaktoren auf die Investitionen.

In den Jahren 1978 bis 1981 übte der Gesamthaushalt einen wachsenden fiskalischen Impuls aus (1977: 7,6 Mrd. DM; 1981: 46,9 Mrd. DM). ${ }^{143}$ Nach der oben vorgenommenen Klassifikation dieses Zeitraums als „klassisch“ hat er sich damit jedoch zumindest im Zeitraum 1977 bis 1979 prozyklisch verhalten. Zur Bekämpfung der auf den zweiten Ölpreisschock folgenden Rezession beschlo $B$ die Bundesregierung in Abstimmung mit der französischen Regierung ein Kreditprogramm zur Förderung der Investitionen vor allem im Energiebereich. Die Kreditanstalt sollte danach 6,3 Mrd. DM auf

141 Vgl. Deutsche Bundesbank (1977), S. $41 \mathrm{ff}$.

142 Vgl. Glastetter/Paulert/Spörel (1983), S. 281-289.

143 Vgl. Sachverständigenrat zur Begutachtung der gesamtwirtschaftlichen Entwicklung (1984), S. 133 ff. Aus methodischen Gründen sind die Angaben mit den 0.g. Zahlen nur begrenzt vergleichbar. In späteren Berechnungen kommt der Sachverständigenrat insgesamt zu niedrigeren Werten. Die aufgezeigten Tendenzen bleiben davon jedoch unberührt. 
den internationalen Kapitalmärkten aufnehmen und daraus zinsverbilligte Darlehen bevorzugt an kleine und mittlere Unternehmen gewähren. Im Februar 1982 beschloß sie darüber hinaus eine „Gemeinschaftsinitiative für Arbeitsplätze, Wachstum und Stabilität", dessen Kern in der Einführung einer befristeten Investitionszulage in Höhe von $10 \%$ bestand, die für Investitionen gewährt wurden, die den Durchschnitt der vorangegangenen drei Jahre überschritten und für die 1982 der Auftrag erteilt bzw. die Baugenehmigung gestellt wurde. Im Rahmen der „Operation ' 82 “ werden zudem Abschreibungserleichterungen sowie Steuererleichterungen für Stahlunternehmen gewährt. Gleichzeitig werden jedoch die Haushalte gekürzt. Die Sachinvestitionen verzeichnen 1981 und 1982 negative Wachstumsraten. Der Haushalt 1982 übt gegenüber 1981 einen verminderten fiskalischen Impuls aus (41,5 gegenüber 46,9 Mrd DM). ${ }^{144}$ Damit tritt im wesentlichen der gleiche Effekt ein, der bereits für 1975 beschrieben wurde. Die Kürzung des Haushalts in Verbindung mit einer wiederum restriktiven Geldpolitik führte erneut zu einem - diesmal jedoch drastischeren - Anstieg der Arbeitslosigkeit. $^{145}$

Neben den bisher beschriebenen Maßnahmen wurde eine Reihe Programme beschlossen, die direkt bei den Erwerbslosen ansetzen. Dazu gehörten die Arbeitsförderungsmaßnahmen für jugendliche und schwer vermittelbare Arbeitslose vom Januar 1976, für die insgesamt 300 Mio. DM zur Verfügung gestellt wurden sowie das arbeitsmarktpolitische Programm vom November des gleichen Jahres, welches Mittel für Arbeitsbeschaffungsmaßnahmen, Berufsbildungsmaßnahmen für Jugendliche, Mobilitätshilfen, Umzugs- und Einrichtungsbeihilfen sowie Eingliederungsmaßnahmen und arbeitsfördernde Maßnahmen für Schwerbehinderte im Umfang von insgesamt 430 Mio. DM vorsah. 1979 wird ein weiteres Sonderprogramm zur Bekämpfung der Arbeitslosigkeit aufgelegt. Mit Hilfe von Lohnkostenzuschüssen im Gesamtvolumen von 500 Mio. DM sollen Gebiete mit überdurchschnittlich hoher Arbeitslosenquote gefördert werden. Einige weitere Maßnahmen wurden bereits oben genannt. ${ }^{146}$ Diese Programme berührten damit einen Bereich, der

144 Vgl. Sachverständigenrat zur Begutachtung der gesamtwirtschaftichen Entwicklung (1984), S. 133 ff., siehe auch Fußnote 143.

145

146

Dies gilt auch für die meisten anderen OECD-Länder.

Bedingt können hierzu auch die Zuschüsse zu den Forschungs- und Personalkosten kleinerer und mittlerer Unternehmen, die im November 1978 beschlossen wurden, 
nach der ursprünglichen beschäftigungspolitischen Konzeption eher dem Arbeitsförderungsgesetz zuzuordnen war. Mit dem Anstieg der Massenarbeitslosigkeit war das Gesetz jedoch völlig überfordert, weil es dafür auch gar nicht vorgesehen war. Mittel, die eigentlich für eine aktive Beschäftigungspolitik vorgesehen waren, wurden nun in Form von Arbeitslosengeld verausgabt. Die zusätzlich zu den Maßnahmen des AFG getroffenen arbeitsmarktpolitischen Programme des Bundes schufen damit einen gewissen Ausgleich. Im Gegensatz zu global wirkenden Maßnahmen waren sie zudem potentiell geeignet, dem Problem der Langzeitarbeitslosigkeit und damit einer hysteretischen Entwicklung der Arbeitslosigkeit entgegenzuwirken. Das Sonderprogramm '79 setzte sich beispielsweise aus den Schwerpunkten „Berufliche Qualifizierung der Arbeitnehmer in Betrieben mit Anpassungs- und Umstellungsprozessen“, „Wiedereingliederung ungelernter sowie längerfristig Arbeitsloser" und „Arbeitsbeschaffungsmaßnahmen zur Verbesserung der sozialen Dienste, des Umweltschutzes und des Wohnumfeldes" zusammen. Die Ergebnisse der umfangreichen Begleitforschung zu diesem Programm bestätigen einen durchaus positiven Effekt (quantitativ und qualitativ), so $\mathrm{da} ß$ die Reduzierung der Arbeitslosigkeit in den geförderten Gebieten zumindest teilweise auf das Sonderprogramm zurückgeführt werden kann. ${ }^{147}$

Insgesamt muß aber festgestellt werden, daß, gemessen am Gesamtvolumen der einzelnen Programme, der beschäftigungspolitische Erfolg eher bescheiden ausfiel. 1975 wurde das grundsätzlich adäquate Mittel der Nachfragestützung durch Geldpolitik und Reallohneinflüsse konterkariert. Ohne die staatlichen Maßnahmen wäre die Arbeitslosigkeit jedoch vermutlich noch höher ausgefallen. Der in der Folge einer anhaltenden Arbeitslosigkeit vollzogene Wechsel des Instrumentariums zog jedoch keine nachhaltige Verbesserung der Beschäftigungssituation nach sich. Eine Ausnahme bildeten die zuletzt beschriebenen auf der Mikroebene angesiedelten Programme.

Die im Zuge all dieser Maßnahmen gewachsenen Defizite machten eine Konsolidierung auf Dauer unumgänglich. In einer Situation, in der eigentlich eine Stützung der Nachfrage erforderlich gewesen wäre, wurde so der not-

sowie das Programm zur Förderung von Existenzgründungen vom Februar des folgenden Jahres gezählt werden.

147 Vgl. etwa Scharpf/Garlichs/Maier/Maier (1982); einen knappen Überblick gibt Ohndorf (1992). 
wendige fiskalische Impuls gekappt. Die (zu) hohen fiskalischen Belastungen einer nur teilweise erfolgreichen Bekämpfung der Arbeitslosigkeit und der damit entstandene Konsolidierungsdruck haben also letztlich den Einsatz besserer Beschäftigungspolitik verhindert. ${ }^{148}$

148 Betrachtet man die arbeitsmarktpolitischen Maßnahmen des AFG isoliert, dann ist ein prozyklisches Verhalten zu konstatieren; vgl. Webber (1982). So wurden bspw. mit dem Arbeitsförderungskonsolidierungsgesetz (AFKG) im Rahmen der „Operation ' 82 " auch Kürzungen bei den arbeitsmarktpolitischen Maßnahmen (i.e.S.) vorgenommen. 
Monika Hanswillemenke and Bernd Rahmann - 978-3-631-75268-5

Downloaded from PubFactory at 01/11/2019 06:29:54AM

via free access 


\section{Beurteilung des Deficit Spending in der sozial-liberalen Koalition aus langfristiger Sicht}

\subsection{Die Entwicklung der Schuldenindikatoren}

Die Entwicklung der Defizite und des Schuldenstandes gibt für sich genommen noch wenig Aufschluß über die Verschuldungssituation der öffentlichen Hand. Häufig werden daher die für die Verschuldung relevanten Größen in Beziehung zueinander sowie in Beziehung zu anderen Größen wie Steueraufkommen, Staatsausgaben und Bruttosozialprodukt gesetzt. Die am häufigsten verwendeten Indikatoren sind in Tabelle 2 zusammengefaßt. So zeigt sich, daß die sog. Schuldenquote (Schulden in v.H. des Sozialprodukts) ihren ersten nachhaltigen Schub Mitte der 70er Jahre erhalten hat. Der durch die Bekämpfung der Rezession 1967/68 verursachte vorläufige Höchststand von 9,5\% (Bund) bzw. 22,3\% (Gesamthaushalt) im Jahre 1968 konnte zunächst durch die Rückführung der Defizite sowie begünstigt durch ein hohes Sozialproduktswachstum zu Beginn der 70er wieder abgebaut werden. Der Bund erreichte 1973 mit 6,2\% sogar ein Niveau, das deutlich unterhalb dessen der 60er Jahre lag. Der durch die Rezession 1975 ausgelöste Anstieg der Schuldenquote wurde in der Folgezeit jedoch nicht in gleicher Weise umgekehrt, sondern war der Beginn zu einer fortwährenden Erhöhung, die bis heute anhält.

Die wachsenden Kosten der Verschuldung schlagen sich beispielsweise in der Zins-Ausgaben- bzw. Zins-Steuerquote (Zinsen in v.H. der Staatsausgaben bzw. der Steuern) nieder. Beide Indikatoren verzeichnen ebenfalls eine steigende Tendenz seit Mitte der 70er Jahre, die sich bis zur Mitte der 80er Jahre stetig fortsetzte. $\mathrm{Zu}$ Beginn der 70er Jahre machen die Zinsausgaben beim Bund im Schnitt knapp 2,7\% der Gesamtausgaben aus. Bis zum Beginn der nächsten Dekade hatte sich ihr Anteil auf 6,4\% erhöht und damit mehr als verdoppelt. Gleichzeitig stieg der Anteil der Steuereinnahmen, der zur Finanzierung der Zinsen notwendig gewesen wäre, bis 1980 auf 7,9\% an. Am Anfang der Regierungsperiode der sozial-liberalen Koalition lag er noch im Bereich von 2,7-2,9\%. Die Kreditfinanzierungsquote (Nettokreditaufnahme in v.H. der Gesamtausgaben) zeigt ebenfalls, daß der Rückgriff auf Kreditfinanzierung zwar durch die antizyklische Haushaltspolitik in den beiden ölpreisbedingten Rezessionen Mitte und Ende der 70er Jahre beson- 
Tabelle 2: Indikatoren zur Verschuldung

\begin{tabular}{|c|c|c|c|c|c|c|c|c|c|c|c|}
\hline \multirow[t]{2}{*}{ Jahr } & \multicolumn{2}{|c|}{$\begin{array}{l}\text { Schulden- } \\
\text { quote }^{1}\end{array}$} & \multicolumn{2}{|c|}{$\begin{array}{c}\text { Kredit- } \\
\text { finanzierungs- } \\
\text { quote }^{2}\end{array}$} & \multicolumn{2}{|c|}{$\begin{array}{c}\text { Zins- } \\
\text { Ausgaben- }^{\text {quote }} \\
\text { quot }^{3}\end{array}$} & \multicolumn{2}{|c|}{$\begin{array}{l}\text { Zins- } \\
\text { Steuer- } \\
\text { quote }^{4}\end{array}$} & \multicolumn{2}{|c|}{$\begin{array}{l}\text { durchschnittl. } \\
\text { Verzinsung d. } \\
\text { Staatsschuld }^{5}\end{array}$} & \multirow{2}{*}{$\begin{array}{l}\text { zum Vergleich: } \\
\text { Wachstum des } \\
\text { nominellen BSP }\end{array}$} \\
\hline & Bund & $\begin{array}{l}\text { Gesamt- } \\
\text { haushalt }\end{array}$ & Bund & $\begin{array}{l}\text { Gesamt- } \\
\text { haushalt }\end{array}$ & Bund & $\begin{array}{l}\text { Gesamt- } \\
\text { haushalt }\end{array}$ & Bund & $\begin{array}{l}\text { Gesamt- } \\
\text { haushalt }\end{array}$ & Bund & $\begin{array}{l}\text { Gesamt- } \\
\text { haushalt }\end{array}$ & \\
\hline 1965 & 7,7 & 18,4 & 1,6 & 5,6 & 1,8 & 2,5 & 1,9 & 3,3 & 3,4 & 4,7 & 9,2 \\
\hline 1966 & 7,8 & 19,3 & 1,7 & 4,6 & 2,2 & 3,0 & 2,4 & 3,9 & 4,3 & 5,3 & 6,4 \\
\hline 1967 & 9,4 & 22,2 & 10,5 & 9,2 & 2,7 & 3,6 & 3,2 & 4,8 & 5,5 & 5,9 & 1,3 \\
\hline 1968 & 9,5 & 22,3 & 7,6 & 6,7 & 2,5 & 3,6 & 2,8 & 4,7 & 4,1 & 5,2 & 8,1 \\
\hline 1969 & 8,4 & 20,2 & 0,0 & 1,4 & 2,7 & 3,6 & 2,8 & 4,3 & 4,3 & 5,3 & 12,0 \\
\hline 1970 & 7,1 & 18,2 & 1,3 & 3,2 & 2,8 & 3,5 & 2,9 & 4,4 & 4,9 & 5,7 & 13,0 \\
\hline 1971 & 6,6 & 18,3 & 1,5 & 6,1 & 2,6 & 3,4 & 2,8 & 4,5 & 5,4 & 6,3 & 11,1 \\
\hline 1972 & 6,6 & 18,7 & 4,4 & 6,5 & 2,5 & 3,5 & 2,7 & 4,5 & 5,6 & 6,4 & 9,9 \\
\hline 1973 & 6,2 & 18,1 & 2,2 & 4,1 & 2,7 & 3,8 & 2,9 & 4,7 & 6,1 & 6,8 & 11,4 \\
\hline 1974 & 7,1 & 18,6 & 7,0 & 7,1 & 3,1 & 3,9 & 3,5 & 5,2 & 7,4 & 7,5 & 7,1 \\
\hline 1975 & 10,4 & 24,1 & 18,7 & 15,1 & 3,3 & 4,1 & 4,3 & 6,1 & 7,5 & 7,9 & 4,5 \\
\hline 1976 & 11,2 & 25,5 & 18,0 & 12,6 & 4,1 & 4,8 & 5,2 & 6,8 & 6,4 & 7,2 & 9,4 \\
\hline 1977 & 12,4 & 26,6 & 14,8 & 8,2 & 4,9 & 5,3 & 5,9 & 7,0 & 6,8 & 7,2 & 6,4 \\
\hline 1978 & 13,7 & 28,0 & 11,4 & 9,6 & 5,0 & 5,1 & 6,2 & 7,0 & 6,5 & 6,8 & 7,9 \\
\hline 1979 & 14,5 & 29,0 & 12,7 & 9,5 & 5,5 & 5,4 & 6,7 & 7,4 & 6,4 & 6,8 & 8,1 \\
\hline 1980 & 15,6 & 30,9 & 11,8 & 10,8 & 6,4 & 5,9 & 7,9 & 8,2 & 6,9 & 7,2 & 6,0 \\
\hline 1981 & 17,5 & 34,3 & 11,5 & 13,2 & 7,7 & 6,8 & 9,9 & 10,1 & 7,8 & 7,9 & 4,2 \\
\hline
\end{tabular}




\begin{tabular}{|r|r|r|r|r|r|r|r|r|r|r|r|}
1982 & 19,4 & 37,7 & 15,2 & 12,4 & 9,0 & 8,1 & 12,0 & 12,2 & 8,2 & 8,4 & 3,3 \\
1983 & 20,4 & 39,3 & 14,9 & 10,1 & 10,7 & 9,2 & 13,9 & 13,3 & 8,6 & 8,5 & 5,4 \\
1984 & 20,7 & 39,9 & 12,4 & 8,8 & 10,9 & 9,4 & 14,0 & 13,2 & 8,1 & 8,1 & 5,2 \\
1985 & 21,4 & 40,8 & 10,9 & 6,9 & 11,2 & 9,4 & 14,0 & 13,1 & 8,0 & 7,9 & 4,0 \\
1986 & 21,4 & 40,6 & 8,7 & 6,8 & 11,5 & 9,4 & 14,4 & 13,2 & 7,7 & 7,7 & 5,5 \\
1987 & 22,0 & 41,8 & 10,1 & 7,7 & 11,4 & 9,2 & 14,2 & 12,9 & 7,5 & 7,4 & 3,5 \\
1988 & 22,5 & 42,3 & 12,7 & 8,6 & 11,6 & 9,3 & 14,5 & 12,9 & 7,3 & 7,2 & 5,2 \\
1989 & 21,8 & 40,7 & 4,8 & 4,2 & 11,0 & 9,0 & 12,9 & 11,8 & 6,8 & 6,8 & 6,7 \\
1990 & 22,1 & 42,5 & 9,3 & 9,7 & 11,0 & 8,6 & 13,1 & 12,2 & 7,0 & 7,0 & 8,9 \\
1991 & 20,3 & 40,1 & 12,5 & 12,8 & 9,8 & 8,6 & 12,3 & 12,5 & 7,3 & 7,4 & 9,0 \\
1992 & 19,6 & 42,7 & 7,2 & 10,4 & 10,2 & 9,7 & 12,3 & 14,3 & 7,5 & 8,7 & 7,4 \\
1993 & 21,7 & 46,9 & 13,0 & 12,1 & 9,9 & 9,4 & 12,7 & 14,2 & 7,5 & 7,7 & 2,2 \\
1994 & 21,5 & 49,0 & 8,1 & 8,0 & 11,1 & 10,1 & 13,7 & 15,2 & 7,7 & 7,7 & 4,8 \\
1995 & 21,9 & 56,7 & 7,9 & 8,1 & 10,1 & 11,1 & 12,7 & 16,5 & 7,0 & 7,9 & 4,1 \\
\hline
\end{tabular}

Schuldenstand am Jahresende in \% des Bruttosozialprodukts

2 Nettokreditaufnahme der $\varnothing$ ffentlichen Haushalte in \% der Gesamtausgaben

${ }^{3}$ Zinsausgaben in \% der Gesamtausgaben

${ }^{4}$ Zinsausgaben in \% des Steueraufkommens

${ }_{6}^{5}$ Zinsausgaben in \% des Schuldenstands am Ende des Vorjahres

${ }^{6}$ Wachstumsrate für das alte Bundesgebiet

Quelle: Statistisches Bundesamt (1994a, 1994b, 1995) sowie interne Unterlagen, Sachverstăndigenrat zur Begutachtung der gesamtwirtschaftlichen Entwicklung (1995, 1996); eigene Berechnungen. 
ders ausgeprägt war. Im Verlauf der 70er Jahre hat sich aber auch hier eine Niveauverschiebung nach oben ergeben.

Diese Maßzahlen gewähren aber nur einen ersten Einblick. So betrachtet geben sie noch keinen Aufschluß über den möglichen Handlungsbedarf der Gebietskörperschaften bzw. des Bundes zur Konsolidierung der Haushalte. Eine detailliertere Analyse der Schuldendynamik, die derartige Fragen besser beantworten kann, soll anhand des Konzeptes der Tragfähigkeit („Sustainability") der öffentlichen Verschuldung erfolgen. Dieses Konzept wurde vor allem von Oliver J. Blanchard Anfang der 90er Jahre für die OECD entwickelt und hat sich rasch als eine mittlerweile übliche Methode zur Untersuchung (und Bewertung) der öffentlichen Verschuldung etabliert. In der konzeptionellen Betrachtung ist dies die Fortsetzung der Betrachtung der Stabilitätseigenschaften eines (sozio-)ökonomischen Systems. Systematisch werden die Voraussetzungen für eine langfristig stabile Entwicklung einer Volkswirtschaft mit öffentlicher Verschuldung erarbeitet. Praktisch wird damit die Frage nach den systemimmanenten Bedingungen für die Erhaltung der Funktionsfähigkeit einer Volkswirtschaft gestellt. 


\subsection{Zur Dynamik der Verschuldung}

\subsubsection{Zins-Wachstums-Differentiale}

Zwischen dem Zinsniveau und den Entwicklungspfaden von Defizit- und Schuldenquote läßt sich ein funktionaler Zusammenhang herstellen, der wiederum in enger Verbindung mit der gesamtwirtschaftlichen Entwicklung (Wachstum) steht. Eine erste modelltheoretische Betrachtung hierzu geht bereits auf Domar ${ }^{149}$ zurück. Er zeigt, daß die volkswirtschaftliche Last der öffentlichen Schulden nicht allein von der Höhe des Schuldenstandes abhängt, sondern entscheidend durch die Wachstumsrate des Sozialprodukts beeinflußt wird.

Vor dem Hintergrund der seit den 70er Jahren wachsenden Staatsverschuldung in den meisten Industrie- und Entwicklungsländern wurden Domars Überlegungen wieder aufgegriffen und weiterentwickelt. ${ }^{150}$ Der im folgenden beschriebene Tragfähigkeitsansatz von Blanchard steht somit in der Tradition von Domar, ist jedoch entscheidend modifiziert und erweitert worden und insofern aussagefähiger als das Domar-Modell.

Ausgangspunkt der Betrachtung ist die sog. Budgetidentität, die schlicht besagt, daß am Ende einer jeden Rechnungsperiode die Ausgaben des Staates seinen Einnahmen entsprechen müssen.

$$
G_{t}+i_{t} * B_{t-1}=T_{t}+\left(B_{t}-B_{t-1}\right)
$$

mit $G_{t}=$ Staatsausgaben ohne Zinszahlungen in der Periode $t$, $\mathrm{T}_{\mathrm{t}}=$ Einnahmen in der Periode $\mathrm{t}$,

$B_{t}$ bzw. $B_{t-1}=$ öffentliche Schulden am Ende der Periode $t$ bzw. $t-1$, und $\quad i_{t}=$ nominale Verzinsung der öffentlichen Schuld in der Periode $t$.

149 Siehe Domar (1944).

150 Vgl. Blanchard (1990), Blanchard/Chouraqui/Hagemann/Sartor (1990), Chouraqui/ Hagemann/Sartor (1990). Für die hier gewählte diskrete Darstellungsweise vgl. Hamilton/Flavin (1986), Heinemann (1993), Joines (1991). 
Im wesentlichen hat der Fiskus drei Möglichkeiten, um seine Ausgaben zu decken: die Erhebung von Steuern, die Finanzierung über den Kreditmarkt oder über eine Veränderung der Geldbasis. Die letzte Möglichkeit, die unter der Bezeichnung Seigniorage in der Literatur diskutiert wird, soll hier zunächst vernachlässigt werden; sie wird weiter unten diskutiert. Darüber hinaus bestehen Einnahmequellen wie Gebühren und Beiträge, Erwerbseinkünfte aus Unternehmen und Gewinne aus Beteiligungen. Diese sonstigen Einnahmen werden zunächst ebenfalls nicht gesondert betrachtet.

Die in der Budgetidentität betrachteten Einnahmen des Fiskus setzen sich also zusammen aus den Steuer- und sonstigen laufenden Einnahmen und der Nettoneuverschuldung, welche der Veränderung des Schuldenstandes am Ende des jeweiligen Haushaltsjahres $\left(B_{t}-B_{t-1}\right)$ entspricht. Dem gegenüber stehen die Staatsausgaben, die sich im Rahmen der laufenden Rechnung im wesentlichen zusammensetzen aus Personalausgaben, laufendem Sachaufwand sowie laufenden Zuweisungen und Zuschüssen, bei der Kapitalrechnung sind insbesondere die Sachinvestitionen und Vermögensübertragungen zu nennen. Darüber hinaus ist jedoch zu beachten, daß die aufgenommenen Kreditmittel nicht nur nicht, wie die Steuern, dauerhaft beim Staat verbleiben, sondern zurückzuzahlen sind und daß zusätzlich zu den eigentlichen Staatsausgaben Zinsen auf den bereits akkumulierten Schuldenstand entrichtet werden müssen, die ebenfalls zu finanzieren sind. Die Nettoneuverschuldung muß damit nicht nur die Lücke zwischen Staatsausgaben (ohne Zinsaufwand) und laufenden Einnahmen, das sog. Primärdefizit $\left(G_{t}-T_{t}\right)$, sondern auch die durch sie selbst verursachten Zinsausgaben $\mathrm{i}_{\mathrm{t}} \cdot \mathrm{B}_{\mathrm{t}-1}$ decken. Die Verschuldung erhält damit eine mögliche Eigendynamik, die im Falle eines permanenten Defizits unter bestimmten Bedingungen zu einer Explosion der Schuldenquote führt und so den ursprünglich gewonnenen zusätzlichen finanzpolitischen Handlungsspielraum auf Dauer wieder einschränkt.

\section{Die Auflösung der Gleichung (1) nach der Nettoneuverschuldung ergibt ${ }^{151}$}

151 Es handelt sich hierbei um eine Bruttodarstellung. Im Falle einer Nettodarstellung müßte der Schuldenstand um das Vermögen des Staates reduziert werden sowie die Zinsausgaben entsprechend um die Zinseinnahmen. In der Bundesrepublik gibt es jedoch keine der Schuldenstatistik äquivalente Vermögensaufstellung. Zudem müßte zunächst einmal der Vermögensbegriff geklärt werden, was schon auf nahezu unüberwindliche Schwierigkeiten stößt. 


$$
\left(B_{t}-B_{t-1}\right)=\left(G_{t}-T_{t}\right)+i_{t} \cdot B_{t-1}
$$

Diese Veränderung des Schuldenstandes entspricht dem sog. Sekundärdefizit, d.h. der Summe aus Primärdefizit und Zinsausgaben. Durch weiteres Auflösen von (2) nach $\mathrm{B}_{t}$ oder $\mathrm{B}_{\mathrm{t}^{-1}}$ und rekursives Einsetzen der laufenden jährlichen Werte für die betrachteten Variablen läßt sich der Schuldenstand zum Zeitpunkt n bestimmen:

$$
B_{n}=B_{0} \cdot \prod_{j=1}^{n}\left(1+i_{j}\right)+\sum_{t=1}^{n}\left[\left(G_{t}-T_{t}\right) \cdot \prod_{j=t+1}^{n}\left(1+i_{j}\right)\right]
$$

Der bis zum zukünftigen Zeitpunkt $\mathbf{n}$ aufgelaufene Schuldenstand setzt sich also zusammen aus dem gegenwärtig bereits bestehenden Schuldenstand $\mathrm{B}_{0}$, der bis zum Zeitpunkt $\mathrm{n}$ aufgezinst wird, und der Summe der von heute bis dahin anfallenden Defizite, ebenfalls jeweils vom Zeitpunkt des Entstehens aufgezinst. Der Zinssatz erweist sich damit neben dem Primärdefizit als zentrale Größe für die Entwicklung der öffentlichen Schulden. ${ }^{152}$

Der absolute öffentliche Schuldenstand ist für sich genommen jedoch wenig aussagekräftig. Relevant ist das Verhältnis des Schuldenstandes zu anderen ökonomischen Variablen, die die wirtschaftliche Leistungsfähigkeit eines Landes ausdrücken, wie z.B. Bruttoinlandsprodukt, Bruttosozialprodukt, Ressourcen im Bankensystem. Ein Land mit hohem Wachstum kann sich eine höhere Verschuldung leisten als ein Land mit schwacher Wirtschaftsleistung, da sowohl die Steuerbasis als auch die privaten Ersparnisse positiv von der Wirtschaftskraft abhängen. Die der Tragfähigkeitsanalyse zugrundegelegte Größe ist daher der Bestand der öffentlichen Schulden in Relation zum Bruttosozialprodukt (Schuldenquote).

152 Man beachte, daß hier jährlich verschiedene Werte angenommen werden. Bei konstantem Zinssatz vereinfacht sich der Ausdruck $\prod_{j=1}^{n}\left(1+i_{j}\right)$ bekanntlich zu $(1+i)^{n}$. 
Analog zu (2) und (3) erhält man für die Schuldenquote ${ }^{153}$ :

$$
b_{t}-b_{t-1}=\left(g_{t}-\tau_{t}\right)+\frac{i_{t}-\theta_{t}}{1+\theta_{t}} \cdot b_{t-1}
$$

und

$$
b_{n}=b_{0} \cdot \prod_{j=1}^{n}\left(\frac{1+i_{j}}{1+\theta_{j}}\right)+\sum_{t=1}^{n}\left[\left(g_{t}-\tau_{t}\right) \cdot \prod_{j=t+1}^{n}\left(\frac{1+i_{j}}{1+\theta_{j}}\right)\right]
$$

mit $b_{t}$ bzw. $b_{t-1}=$ Schuldenquote (Staatsschuld in v.H. des BSP) in der Periode $\mathrm{t}$ bzw. $\mathrm{t}-1$,

$\mathrm{g}_{\mathrm{t}}=$ Ausgabenquote (Staatsausgaben in v.H. des BSP) in der Periode $\mathrm{t}$,

$\tau_{\mathrm{t}}=$ Einnahmenquote (Einnahmen in v.H. des BSP) in der Periode $\mathrm{t}$, und $\theta_{\mathrm{t}}=$ Wachstumsrate des nominellen BSP in der Periode $\mathrm{t}$.

Da i und $\theta$ jeweils kleine Werte sind, läßt sich (5a) wie folgt approximieren $^{154}$ :

153 Division von (2) durch $Y_{t}$ (= Bruttosozialprodukt in der Periode $t$ ) ergibt

$\frac{B_{t}}{Y_{t}}-\frac{B_{t-1}}{Y_{t}}=\frac{G_{t}}{Y_{t}}-\frac{T_{t}}{Y_{t}}+\frac{i_{t} \cdot B_{t-1}}{Y_{t}}$

Weiterhin gilt

$$
\frac{B_{t}}{Y_{t}}-\frac{B_{t-1}}{\left(1+\theta_{t}\right) \cdot Y_{t-1}}=\frac{G_{t}}{Y_{t}}-\frac{T_{t}}{Y_{t}}+\frac{i_{t} \cdot B_{t-1}}{\left(1+\theta_{t}\right) \cdot Y_{t-1}}
$$

Nach weiteren Umformungen und Übergang zu Quoten erhält man Gleichung (4). Auflösen von (4) nach $b_{t}$ oder $b_{t-1}$ und rekursives Einsetzen führen zu (5a).

154 Durch Erweiterung von $\left(\frac{1+i_{j}}{1+\theta_{t}}\right)$ mit $\left(1-\theta_{j}\right)$ und Ausmultiplizieren erhält man $\frac{1+i_{j}-\theta_{j}-i_{j} \cdot \theta_{j}}{1-\theta_{j}^{2}}$. Da $i_{j}$ und $\theta_{j}$ jeweils kleine Werte sind, liegen $i_{j} \cdot \theta_{j}$ sowie $\theta_{\mathrm{j}}{ }^{2}$ nahe Null und können vernachlässigt werden. Mithin ergibt sich $\left(1+\mathrm{i}_{\mathrm{j}}-\theta_{\mathrm{j}}\right)$. 


$$
b_{n}=b_{0} \cdot \prod_{j=1}^{n}\left(1+i_{j}-\theta_{j}\right)+\sum_{t=1}^{n}\left[\left(g_{t}-\tau_{t}\right) \cdot \prod_{j=t+1}^{n}\left(1+i_{j}-\theta_{j}\right)\right]
$$

Die zukünftige Schuldenquote setzt sich zusammen aus der aufgezinsten heutigen Schuldenquote zuzüglich der Summe der jeweils aufgezinsten Defizitquote. Ausschlaggebend für die Aufzinsung ist jedoch nicht mehr allein der Zinssatz, sondern die Differenz aus Zinssatz und Wachstumsrate des Sozialprodukts, das sog. Zins-Wachstums-Differential. Die Entwicklung der Schuldenquote hängt entscheidend von der Größe und dem Vorzeichen dieses Faktors ab. Grundsätzlich sind drei Fälle zu unterscheiden:

a) Das Wachstum des Sozialprodukts übersteigt den Zinssatz

$$
\left.\left(\theta_{j}>i_{j} \text { bzw. } i_{j}-\theta_{j}<0\right)\right) \text {. }
$$

b) Der Zinssatz liegt über der Wachstumsrate des Sozialprodukts $\left(\theta_{j}<i_{j}\right.$ bzw. $\left.\left.i_{j}-\theta_{j}>0\right)\right)$.

c) Wachstumsrate und Zinssatz stimmen überein $\left(\theta_{j}=i_{j}\right.$ bzw. $\left.\left.i_{j}-\theta_{j}=0\right)\right) .155$

Im ersten Fall (negatives Zins-Wachstums-Differential) kann der Fiskus langfristig eine bestimmte Primärdefizitquote aufrecht erhalten, ohne daß die Schuldenquote explodiert; diese strebt vielmehr einem endlichen Grenzwert zu. Eine derartige Erkenntnis ist auch dem eingangs dieses Abschnittes erwähnten Modell von E.D. Domar zu entnehmen. ${ }^{156}$ Aus dem vergleichsweise hohen Wirtschaftswachstum werden die Zinslasten durch überproportional steigende laufende Einnahmen des Fiskus gedeckt. Die durch die Verschuldung entstehende Zinsbelastung kann gewissermaßen nach Art eines Kettenbriefes revolvierend durch Neuverschuldung finanziert werden. Nach dem ersten Nutzer eines solchen Kettenbriefes, Charles Ponzi, wird diese Vorgehensweise als Ponzi-Finanzierung bezeichnet. Die Schuldenquote strebt langfristig einem Grenzwert zu, so daß sich die Frage der Tragfähig-

155 Es sei hier nochmals darauf hingewiesen, daß es sich sowohl beim rechnerischen 156 Zins als auch bei der Wachstumsrate um nominelle Größen handelt.

Siehe Fußnote 149. 
keit der öffentlichen Verschuldung positiv beantworten läßt. Die an der Wachstumsrate im Vergleich zum Zinssatz gemessene „reiche“ Zukunft ist in der Lage, die Schulden der Vergangenheit zu bedienen, ohne damit ihre eigene Existenz zu gefährden. Dieses Ergebnis, beruhend auf einem Vergleich des Zinssatzes mit der nominellen Wachstumsrate des Sozialprodukts, hatte im Prinzip bereits E.D. Domar im Jahre 1944 (wenngleich auf anderem Wege) modellhaft erarbeitet.

Der beschriebene Zusammenhang kann anhand von Schaubild 5 verdeutlicht werden. Dabei bezeichnet $\mathrm{b}$ auch hier die Schuldenquote, während $\dot{\mathrm{b}}$ die Veränderung der Schuldenquote darstellt. Die Veränderung der Schuldenquote ist, wie gezeigt wurde, neben der Schuldenquote selbst bestimmt durch das Primärdefizit ( $\mathrm{g}-\tau$ ) und das Zins-Wachstums-Differential (i- $\theta$ ), das hier als konstant und negativ angenommen wird. ${ }^{157}$

Als Ausgangssituation sei der Punkt A gewählt, in dem die Schuldenquote konstant $(\mathrm{d} . \mathrm{h} . \dot{\mathrm{b}}=0)$ bei $\mathrm{b}_{0}$ liegt. Dieses Gleichgewicht ist vereinbar mit einem Primärdefizit von $(\mathrm{g}-\tau)_{0}$. Betreibt der Staat nun aus dieser Situation heraus eine expansive Fiskalpolitik, indem er das Primärdefizit erhöht, drückt sich dies in einer Verschiebung der Geraden nach oben aus und die Schuldenquote nimmt $\mathrm{zu}$; der ursprüngliche Gleichgewichtszustand ist zunächst gestört. Bei einem neuen Primärdefizit von $(g-\tau)_{1}$ beispielsweise wird der Zustand direkt nach Ausweitung des Primärdefizits durch den Punkt B beschrieben. Die Erhöhung der Schuldenquote $(\dot{b}>0)$ ist indes nicht dauerhaft, denn wegen der über der Verzinsung liegenden Wachstumsrate wird die Schuldenquote sukzessive zurückgeführt. Dieser Prozeß endet in einem neuen dynamischen Gleichgewicht, wobei die Schuldenquote gegen einen gegenüber der Ausgangslage erhöhten - Wert von $b_{n}$ konvergiert. Entscheidend ist jedoch, daß das beschriebene System unter den getroffenen Annahmen stabil ist.

Betrachtet man die Zeit der sozial-liberalen Koalition vor diesem Hintergrund, so läßt sich folgendes feststellen (vgl. Tabelle 2 und Schaubild 6): In den Jahren 1969 bis 1982 liegt die nominale Wachstumsrate des Sozialpro-

157 Der Grafik liegt der folgende Zusammenhang zugrunde: $\dot{b}=(g-\tau)+(i-\theta) \cdot b$. Dies ist das stetige Äquivalent zu Gleichung (4). 
Schaubild 5:

Schuldendynamik bei negativem Zins-Wachstums-Differential

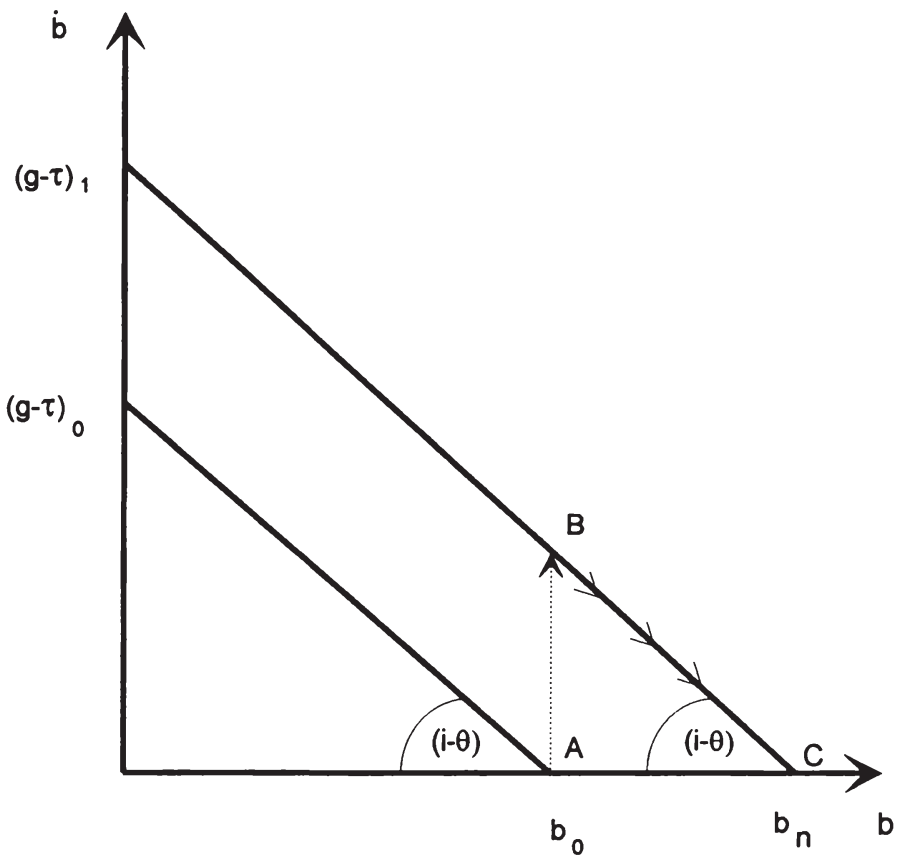

Quelle: In Anlehnung an Lehment (1988).

dukts mit durchschnittlich $8,2 \%$ bis auf wenige Ausnahmejahre über der Verzinsung der Staatsschuld mit einem Durchschnittswert von 7\%. Aufgrund des besseren Zugangs zu den Kapitalmärkten liegt die durchschnittliche Verzinsung der Bundesschuld mit 6,5\% deutlich darunter. Das ZinsWachstums-Differential des Bundes liegt mit einem durchschnittlichen Wert von $-1,7 \%$ damit absolut gesehen sogar noch über dem Durchschnittswert für den Gesamthaushalt von -1,2\%. In der Zeit bis 1980 ist bis auf die Ausnahmejahre der Rezessionen 1967 und 1975 die Lehre aus dem damals bekannten „Domar-Modell“ also die, daß öffentliche Verschuldung keine fis- 
kalische Systemgefahr bedeutet, weil (zum Teil sogar erhebliche) negative Differenzen zwischen Zinssatz und Wachstumsrate beobachtet werden konnten. Erst nach 1975 wird dieses Differential deutlich geringer, bleibt aber negativ. Geht man nun für die 80er Jahre davon aus (und für einen wirtschaftspolitischen Optimisten ist dies durchaus naheliegend), daß sich die Gesamtwirtschaft sowie die Haushalte der Gebietskörperschaften in den Folgejahren im Durchschnitt genauso fortentwickeln würden wie in den 70er Jahren, dann wären Schuldenquoten vom Gesamthaushalt bzw. Bund langfristig auf 116,7 bzw. 47,1\% des Bruttosozialprodukts angestiegen (unter Zugrundelegung einer durchschnittlichen Primärdefizitquote von 1,4 bzw. $0,8 \%$ des Sozialprodukts). Die jährlichen Zinszahlungen hätten sich entsprechend auf 8,2 bzw. 3,1\% des Bruttosozialprodukts erhöht. Schuldenquote und Zinsausgaben erscheinen zwar recht hoch, man muß jedoch bedenken, $\mathrm{da} ß$ sie bei der angenommenen Konstellation nicht weiter gestiegen wären. Von der rein fiskalischen Seite hätte mit solchen Grenzwerten ein Schuldenproblem im Sinne einer drohenden Explosion des Systems folglich nicht existiert. Es ist also kaum verwunderlich, daß die Verschuldung zunächst nicht als Problem angesehen wurde. Ganz abgesehen von der damals noch positiven Beurteilung des Deficit Spending in der Keynesianischen Theorie.

$\mathrm{Da}$ der Zinssatz langfristig und dauerhaft unter der Wachstumsrate des nominellen Bruttosozialprodukts liegen kann, muß jedoch theoretisch als wenig plausibel angesehen werden. So würden in diesem Fall beispielsweise die Aktienkurse von Unternehmen, deren Dividendenzahlungen mit der Rate des Bruttosozialprodukts wachsen, einen Wert von unendlich aufweisen, weil eine kreditfinanzierte Nachfrage nach ihnen einen dauerhaften sicheren nominalen Gewinn verspricht. Es handelt sich sicherlich bewußt um ein konstruiertes Beispiel, das die Tendenz dieser Aussage zu Recht verdeutlicht. Des weiteren müßten Schulden, die im Ausland aufgenommen würden, praktisch niemals zurückgezahlt werden; d.h. ein Land könnte ein permanentes Leistungsbilanzdefizit aufrecht erhalten. ${ }^{158}$ Gerade so, wie die trügerische Hoffnung auf ein Funktionieren der erwähnten Kettenbrief-Methode, ist die Ponzi-Finanzierung auf den unbegründeten Glauben an eine immerwährende Prosperität gegründet. In der Tat hat sich eine solche Entwicklung faktisch auch nicht bestätigt. Für jedes Jahr, in welchem der Zinssatz über der nomi-

$158 \mathrm{Vgl}$. Boss/Lorz (1995), S. 5. 
Schaubild 6

\section{Entwicklung des Zins-Wachstums-Differentials für den Bund} in v.H.

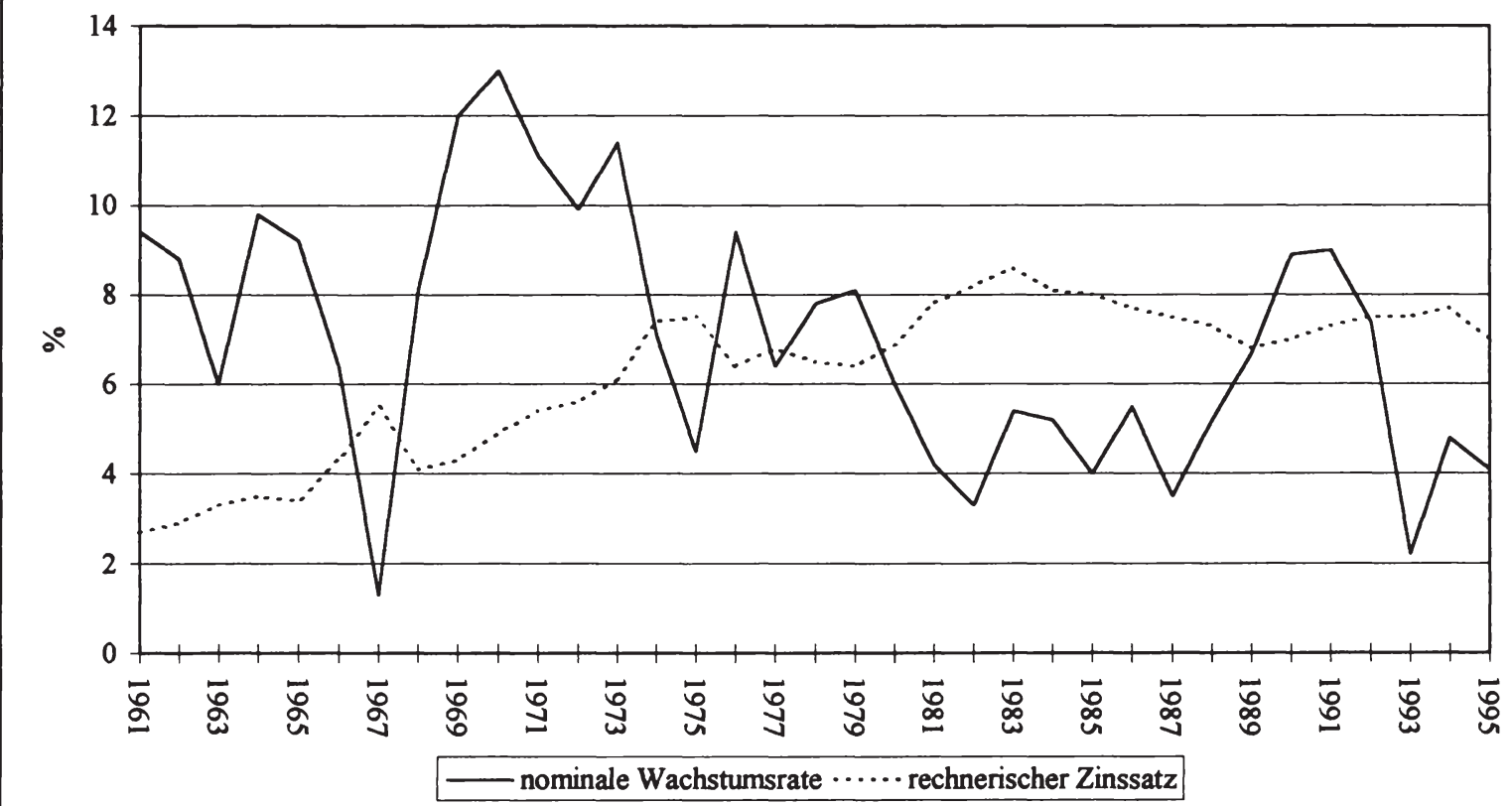


nalen Wachstumsrate liegt, erweist sich die Zins-Wachstums-Mechanik also als fatal, denn der Grenzwert einer langfristigen Schuldenquote wird jedesmal größer; oder mit anderen Worten; langfristig gibt es eben realiter keine festen Grenzwerte. Empirisch hat sich dennoch - und nicht allein in der Bundesrepublik - über einen langen Zeitraum ein relativ niedriger Zinssatz halten können. Ein nicht unwesentlicher Grund für die Abkehr von dieser Entwicklung scheint in der Instrumentalisierung der Zinssätze durch die Notenbanken als Mittel zur Inflationsbekämpfung zu liegen. ${ }^{159}$

Ist der empirisch relevante Fall nunmehr der, in dem der Zinssatz die Wachstumsrate zwar nicht permanent, aber im Durchschnitt auf Dauer gesehen übersteigt $\left(\mathrm{i}_{\mathrm{j}}-\theta_{\mathrm{j}}>0\right)$, strebt die Schuldenquote nicht automatisch einem Grenzwert zu; vielmehr führt ein permanentes Defizit langfristig zu einer Explosion der Schuldenquote. Im Grenzfall der Übereinstimmung von nomineller Wachstumsrate und rechnerischem Zins werden die Zinsausgaben gerade durch das Wachstum finanziert $\left(\mathrm{i}_{\mathrm{j}}-\theta_{\mathrm{j}}=0\right)$. Ein permanentes Defizit führt aber gleichwohl zu einer Explosion der Schuldenquote, wenngleich die Ausdehnung langsamer vonstatten geht als im Falle eines die Wachstumsrate übersteigenden Zinses. Wenn also die für eine günstige Interpretation erforderliche Bedingung eines dauerhaft negativen Zins-Wachstums-Differentials realiter nicht erfüllt ist, müssen zur Stabilisierung einer gegebenen Schuldenquote immer wieder ausgleichende Überschüsse im öffentlichen Haushalt erzielt werden können.

Die beschriebene Dynamik kann anhand von Schaubild 7 verdeutlicht werden. Die Bezeichnungen entsprechen denen in Schaubild 5. Das ZinsWachstums-Differential wird ebenfalls als konstant angenommen, jetzt jedoch als positiv. Als Ausgangssituation sei wiederum der Punkt A gewählt, in dem der Schuldenstand konstant ist $(\dot{b}=0)$ und der Haushalt einen Primärüberschuß (negatives Primärdefizit) von - $(\mathrm{g}-\tau)_{0}$ verzeichnet, denn nur mit einem solchen ist bei gegebenem Zins-Wachstums-Differential die Schuldenquote $b_{0}$ vereinbar. Mit einem Primärüberschuß in dieser Höhe ist es möglich, den defizit- und schuldentreibenden Zinseffekt gerade auszugleichen. Sei nun weiterhin angenommen, daß der Haushalt (aus welchen Gründen auch immer) defizitär wird, z.B. mit einem Primärdefizit von (g- $\tau)_{1}$.

159

Vgl. Pasinetti (1997). 
Schaubild 7:

Schuldendynamik bei positivem Zins-Wachstums-Differential

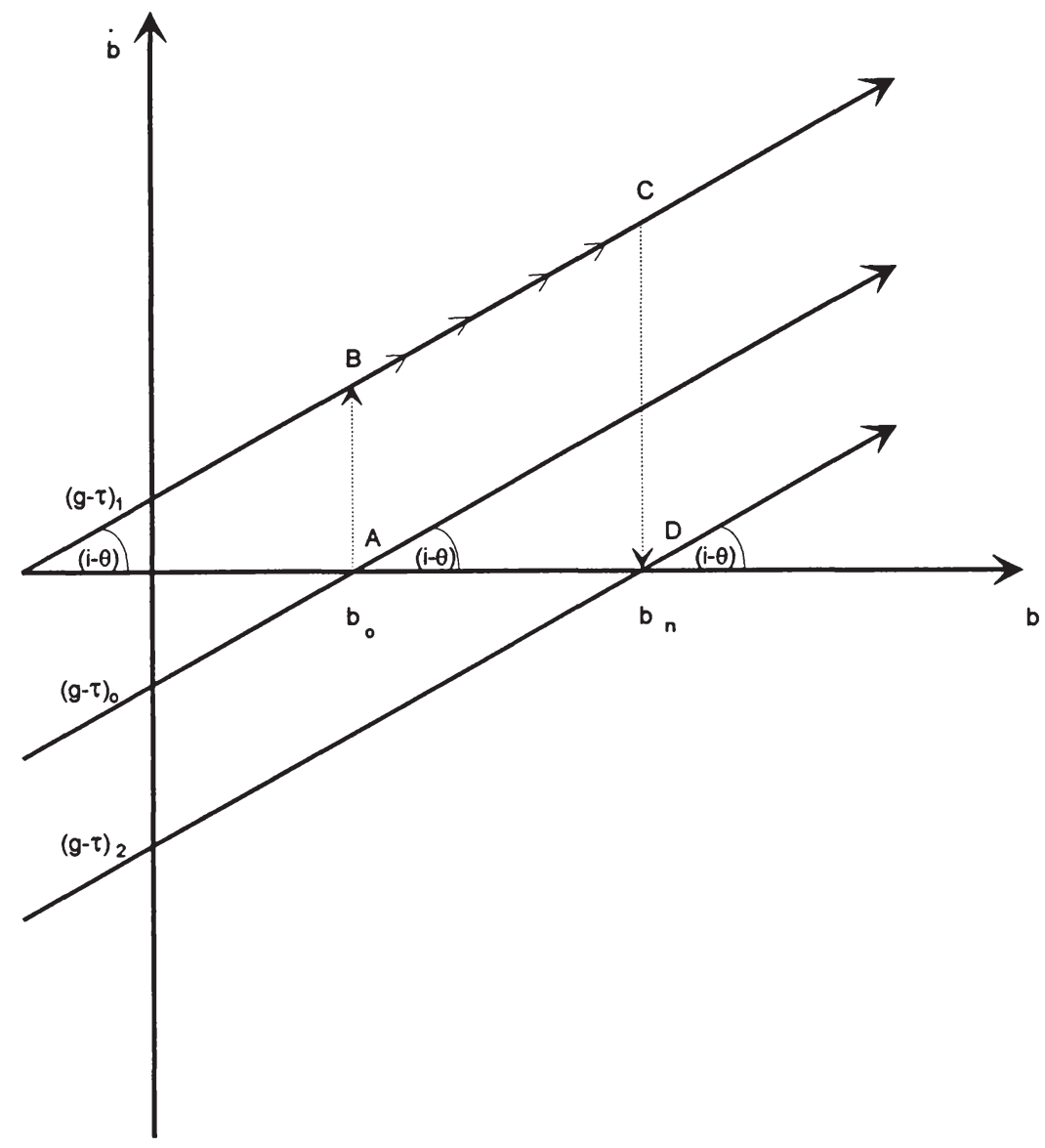

Quelle: In Anlehnung an Lehment (1988). 
Im Schaubild bedeutet dies einen Sprung von A nach B. Hieraus ergibt sich nun eine Erhöhung der Schuldenquote, die sich aufgrund des Zinseffekts auch in Zukunft fortsetzen wird, und zwar um so stärker, je größer die Differenz zwischen Zins und Wachstum ist. Auf Dauer kann diese Entwicklung nur mit Hilfe eines Primärüberschusses gestoppt werden, der jedoch nach Maßgabe der zwischenzeitlich erfolgten Dynamik über das ursprüngliche Niveau hinausgehen muß. Soll die Schuldenquote auf dem im Punkt C erreichten Niveau gehalten werden, dann ist ein permanenter Primärüberschuß von $-(\mathrm{g}-\tau)_{2}$ nötig. Nach erfolgreicher Konsolidierung würde man den Punkt $\mathrm{D}$ erreichen, in dem die Schuldenquote wiederum konstant ist. Um die Schuldenquote auf ihr ursprüngliches Niveau zurückzuführen, wäre ein noch höherer Überschuß vonnöten.

\subsubsection{Finanzierungslücken}

Ausgangspunkt der Überlegungen zur Bedeutung von Zins-Wachstums-Differentialen ist die Budgetidentität, also praktisch die Tautologie, daß am Ende eines Haushaltsjahres Ausgaben und Einnahmen eines Budgets stets gleich hoch sein müssen (Grundsatz des Haushaltsausgleichs nach Art. 110(1) GG). Der Ausgleich wird letztlich durch ungeplante Verschuldungsaktivitäten herbeigeführt, etwa durch Verschuldung bei der Zentralnotenbank mit der Folge der Erhöhung der Geldmenge ${ }^{160}$ oder (und) höhere Kreditaufnahme auf dem Markt bzw. durch Aussetzen von Schuldentilgung. (Im Falle eines ungeplanten Budgetüberschusses drehen sich solche Transaktionen um.) Dies führt dann in der Regel zu einer weiteren Expansion der Schuldenquote, statt diese mindestens zu stabilisieren. Wie gezeigt ist dann nur im Fall dauerhafter negativer Zins-Wachstums-Differentiale eine ,gutartige" Entwicklung zu erwarten, aber eben dieser Fall kann prinzipiell nicht als gegeben unterstellt werden. Empirisch ist er für die 70er Jahre zu beobachten; in den 80er Jahren kehrt sich der Zusammenhang aber nachhaltig um. Es ist also realistisch, von einem überwiegend positiven Zins-Wachstums-Differential auszugehen; d.h. zur Konsolidierung der Verschuldungssituation müssen bestimmte Einnahmen erzielt werden (bzw. Ausgaben unter-

160 Bei geldpolitisch unabhängigen Notenbanken erfolgt dies auf unterschiedlichen Wegen; bei politischer Abhängigkeit gibt es dazu direktere Möglichkeiten. Darauf soll an dieser Stelle nicht eingegangen werden; vgl. Abschnitt 3.5. 
bleiben), die ein weiteres Anwachsen der Schuldenquote in Zukunft verhindern. Üblicherweise wird angenommen, daß die am Beginn des jeweiligen Beobachtungszeitraums gegebene Schuldenquote $\left(b_{0}\right)$ für einen bestimmten Zeitraum stabilisiert werden soll. Das bedeutet formal, daß

$$
\lim _{n \rightarrow N} b_{n}=b_{0}
$$

gelten soll, wobei unterschiedliche Zeithorizonte $(\mathrm{N})$ unterstellt werden können. Für eine wachsende Schuldenquote bedeutet dies, daß sie bis zu einem bestimmten Zeitpunkt auf das heutige Niveau zurückgeführt werden bzw. erst gar nicht über dieses Niveau hinaus wachsen darf. ${ }^{161}$ Im folgenden wird zwischen kurzfristiger (Stabilisierung zum Ende der laufenden Periode), mittelfristiger (5-Jahreszeitraum) und langfristiger (10-Jahreszeitraum) Betrachtung unterschieden.

Um nun festzustellen, ob die Haushaltspolitik über den jeweiligen Zeitraum tragfähig ist, wird eine dafür notwendige Einnahmenquote $\left(\tau^{*}\right)$ errechnet. Darunter versteht man diejenige Einnahmenquote, die bei gegebener Ausgabenpolitik erzielt werden muß, um die Schuldenquote (auf dem Ausgangsniveau) zu stabilisieren. Sie läßt sich aus (5a) und (6) herleiten ${ }^{162}$ :

161 Natürlich kann man auch eine Schuldenquote stabilisieren, die über derjenigen liegt, die zum Zeitpunkt der Betrachtung realisiert worden ist bzw. realisiert wird. In einem solchen Falle kann sich der Staat zunächst noch weiter verschulden. Von einer solchen Möglichkeit soll jedoch angesichts der bereits in den 70er Jahren hohen Schuldenquote abgesehen werden. Ebenso ist eine Stabilisierung auf einem niedrigeren Niveau denkbar; vgl. hierzu Kapitel 4. Im Vergleich dazu stellt Gleichung (6) eine eher „weiche" Anforderung dar.

Hierzu wird der Grenzwert von ( $5 a$ ) für $n \rightarrow N$ gebildet, $d . h$.

$$
\lim _{n \rightarrow N} b_{n}=\lim _{n \rightarrow N}\left\{b_{0} \cdot \prod_{j=1}^{n}\left(\frac{1+i_{j}}{1+\theta_{j}}\right)+\sum_{t=1}^{n}\left[\left(g_{t}-\tau_{t}\right) \cdot \prod_{j=t+1}^{n}\left(\frac{1+i_{j}}{1+\theta_{j}}\right)\right]\right\}
$$

Unter Berücksichtigung von (6) folgt hieraus

$$
b_{0}=b_{0} \cdot \prod_{j=1}^{N}\left(\frac{1+i_{j}}{1+\theta_{j}}\right)+\sum_{t=1}^{N}\left[\left(g_{t}-\tau_{t}\right) \cdot \prod_{j=t+1}^{N}\left(\frac{1+i_{j}}{1+\theta_{j}}\right)\right] \text {. }
$$

Sei nun $\tau_{t}=\tau$ * für alle $t$ ( $g_{t}$ bleibt variabel, denn es wird diejenige (konstante) Einnahmenquote gesucht, die bei gegebener Ausgabenentwicklung die Schulden- 
(7) $\tau^{*}=\left[\sum_{t=1}^{N} \prod_{j=1}^{t}\left(\frac{1+\theta_{j}}{1+i_{j}}\right)\right]^{-1} \cdot\left\{b_{0}\left[1-\prod_{j=1}^{N}\left(\frac{1+\theta_{j}}{1+i_{j}}\right)\right]+\sum_{t=1}^{N} g_{t} \prod_{j=1}^{t}\left(\frac{1+\theta_{j}}{1+i_{j}}\right)\right\}$

Diese notwendige Einnahmenquote wird dann mit der tatsächlichen Einnahmenquote verglichen. Die Differenz der Werte

$$
\tau^{*}-\tau_{\mathrm{t}}
$$

ist die sog. Einnahmenlücke und dient nun als Indikator dafür, ob und wie starke Anpassungsmaßnahmen im Budget notwendig sind. Ist die Einnahmenlücke positiv, dann kann die Schuldenquote nur stabilisiert werden, wenn der Haushalt konsolidiert wird. Bei $\tau^{*}$ handelt es sich quasi um eine Konstante. Dies darf jedoch nicht darüber hinwegtäuschen, daß sich $\tau^{*}$ jeweils auf einen konkreten Ausgangszeitpunkt bezieht und sich aus der Schulden- und der erwarteten Haushaltssituation zu eben diesem Zeitpunkt ergibt.

Je nach dem Zeitraum, in dem das Konsolidierungsziel erreicht werden soll, wird zwischen kurz-, mittel- und langfristigen Einnahmenlücken unterschieden. Die kurzfristige Einnahmenlücke gibt an, welche Steuererhöhung bzw. Ausgabensenkung (in v.H. des Bruttosozialprodukts) notwendig sind, wenn eine Anpassung sofort erfolgen soll. Die mittel- bzw. langfristigen Einnahmenlücken geben die notwendigen Anpassungen für eine Stabilisierung innerhalb von 5 bzw. 10 Jahren an. Bei kurzfristiger Betrachtung reduziert sich (7) zu

$$
\tau^{*}=b_{t-1} \cdot \frac{i_{t}-\theta_{t}}{1+\theta_{t}}+g_{t}
$$

und für $\left(\tau^{*}-\tau_{t}\right)$ gilt

quote stabilisiert). In vorstehende Gleichung eingesetzt und nach $\tau^{*}$ aufgelöst ergibt sich (7). (Gleichung (7) weicht von der bei Blanchard/Chouraqui/Hagemann/Sartor (1990, S. 35, Fußnote 7) angegebenen diskreten Formel ab.) 


$$
\tau^{*}-\tau_{t}=\left(g_{t}-\tau_{t}\right)+b_{t-1} \cdot \frac{i_{t}-\theta_{t}}{1+\theta_{t}} \cdot{ }^{163}
$$

$\tau^{*}$ muß also gerade so groß sein, um die Ausgaben der (vom Betrachtungszeitraum aus gesehen) laufenden Periode und der über das Bruttosozialproduktswachstum hinausgehende Verzinsung zu finanzieren. Liegen die Einnahmen der laufenden Periode unterhalb von $\tau^{*}$, so ist eine (sofortige) Steuererhöhung und/oder Ausgabensenkung notwendig. Im umgekehrten Fall ist eine Lockerung der Haushaltspolitik denkbar. (Alle Werte sind in Relation zum Sozialprodukt zu sehen.)

Die mittel- und langfristigen Einnahmenlücken unterscheiden sich von den kurzfristigen dadurch, daß

- die Stabilisierung innerhalb von 5 (bzw. 10) Jahren erfolgen soll, also ein größerer Anpassungszeitraum besteht;

- die Ausgaben der laufenden und der nächsten vier (bzw. 9) Jahre (einschließlich Verzinsung) berücksichtigt werden und

- die bereits bestehende Schuldenquote ebenfalls eine Verzinsung über einen längeren Zeitraum erfährt.

Je nach Haushaltspolitik kann eine Verlängerung des Betrachtungszeitraums zu einer Verbesserung oder Verschlechterung des jeweiligen Indikators führen. Auftretende extreme Ergebnisse des Haushalts werden durch eine längerfristige Betrachtung in der Regel geglättet.

Analog zu den Einnahmenlücken lassen sich sog. Ausgabenlücken $\left(\mathrm{g}_{\mathrm{t}} \mathrm{g}^{*}\right)$ oder Primärüberschußlücken $\left(p^{*}-p_{t}\right)$ entwickeln ${ }^{164}$, wobei

$163 \mathrm{Zu}$ dem gleichen Ergebnis gelangt man in diesem Fall, indem man in Gleichung (4) $b_{t}-b_{t-1}=0$ (bzw. $b_{t}=b_{t-1}$ ) setzt, was praktische einer sofortigen Stabilisierung der Schuldenquote gleichkommt.

Die Herleitung von (11) und (12) entspricht der Berechnung von Gleichung (7). Es gilt jedoch nun $g_{t}=g *$ bzw. $p_{t}=p *$ für alle $t$, wobei $p_{t}=\left(\tau_{t}-g_{t}\right)$. 
(11) $g^{*}=\left[\sum_{t=1}^{N} \prod_{j=1}^{t}\left(\frac{1+\theta_{j}}{1+i_{j}}\right)\right]^{-1} \cdot\left\{-b_{0}\left[1-\prod_{j=1}^{N}\left(\frac{1+\theta_{j}}{1+i_{j}}\right)\right]+\sum_{t=1}^{N} \tau_{t} \prod_{j=l}^{t}\left(\frac{1+\theta_{j}}{1+i_{j}}\right)\right\}$ und

$$
\mathrm{p}^{*}=\left[\sum_{\mathrm{t}=1}^{\mathrm{N}} \prod_{\mathrm{j}=1}^{\mathrm{t}}\left(\frac{1+\theta_{\mathrm{j}}}{1+\mathrm{i}_{\mathrm{j}}}\right)\right]^{-1} \cdot\left\{\mathrm{b}_{0}\left[1-\prod_{\mathrm{j}=1}^{\mathrm{N}}\left(\frac{1+\theta_{\mathrm{j}}}{1+\mathrm{i}_{\mathrm{j}}}\right)\right]\right\}
$$

Die Existenz von Einnahmen- oder Ausgabenlücken bedeutet natürlich nicht, da $\beta$ diese einseitig jeweils nur über Steuererhöhungen bzw. Ausgabensenkungen beseitigt werden können. Die als notwendig erachtete Anpassung kann in beiden Fällen über jede Budgetseite erfolgen. Alle hier unterschiedenen Indikatoren beziehen sich allgemein auf den notwendigen Anpassungsbedarf und lassen zunächst offen, auf welcher Seite des Budgets die finanzpolitisch adäquaten Maßnahmen erfolgen sollen. Bei kurzfristiger Betrachtung sind Einnahmen-, Ausgaben- sowie Primärdefizitlücken identisch. 


\subsection{Zur Tragfähigkeit der Verschuldungspolitik}

\subsubsection{Die Grundlagen}

Die Haushaltspolitik der sozial-liberalen Koalition soll nun anhand der oben entwickelten Indikatoren beurteilt werden. Das in der Regel zur Beurteilung der erwarteten zukünftigen Entwicklung der Verschuldung verwendete Tragfähigkeitskonzept dient hier zunächst also einer Ex-post-Betrachtung. Für jedes Jahr des Betrachtungszeitraums werden jeweils die oben beschriebenen Indikatoren ermittelt. Für die Jahre 1969 bis 1982 werden hierzu die tatsächlich realisierten Werte für Einnahmen und Ausgaben herangezogen. In die mittel- bzw. langfristigen Indikatoren der Jahre 1979 bzw. 1974 und der darauffolgenden Jahre gehen jedoch auch Haushaltsdaten der Jahre nach 1982 ein. Da hier jedoch ein Politikwechsel stattfand, können für diese Jahre nicht die tatsächlichen Ausgaben- und Einnahmendaten herangezogen werden. Es ist also notwendig, die aus damaliger Sicht „zukünftigen“ Einnahmen und Ausgaben abzuschätzen, die im Falle einer Fortsetzung der sozial-liberalen Koalition zu erwarten gewesen wären. Hierzu werden die Trends der Jahre 1970 bis 1982 sowie 1976 bis 1982 gebildet und für die Folgejahre extrapoliert. Damit ist zum einen die Einnahmen- und Ausgabenentwicklung über die gesamte Regierungszeit berücksichtigt, zum anderen wird in Rechnung gestellt, daß - wie in Abschnitt 1.2 dargestellt - eine gewisse Trendwende in der Finanzpolitik stattgefunden hat. Die Entwicklung der Einnahmen und Ausgaben sowie deren Relation zum Sozialprodukt (Einnahmen- bzw. Ausgabenquote) sind in Schaubild 8 und 9 dargestellt.

Insbesondere der Verlauf der Quoten legt ebenfalls eine Unterscheidung der Szenarien nach den Zeiträumen 1970 bis 1982 und 1976 bis 1982 nahe bzw. rechtfertigt eine solche Vorgehensweise. Für die Einnahmenlücken (Ausgabenlücken) werden daher die folgenden Szenarien zugrundegelegt:

- Szenario 1 (bzw. 5)

Es wird der Trend der Ausgaben ohne Zinsen (bzw. Einnahmen) für 1970 bis 1982 gebildet und dieser für die Jahre 1983 bis $1991^{165}$ extrapoliert.

165 Zur Berechnung der langfristigen Indikatoren werden Werte bis 1991 benötigt, für die mittelfristigen bis 1986 . 
Die Zeitreihe der Ausgabenquote (bzw. Einnahmenquote) setzt sich damit zusammen aus den tatsächlichen Quoten für die Jahre 1969 bis 1982 und den Quoten auf Basis der Extrapolation für 1983 und die darauffolgenden Jahre, wobei zur Berechnung der Quoten jeweils die tatsächlichen und extrapolierten Ausgaben (bzw. Einnahmen) in Relation gesetzt werden zum tatsächlichen Bruttosozialprodukt.

- Szenario 2 (bzw. 6)

Wie in Szenario 1 (bzw. 5) wird hier der Trend der Ausgaben ohne Zinsen (bzw. Einnahmen) gebildet und für die Folgejahre extrapoliert. Der Trend beruht hier jedoch auf den Daten der Jahre 1976 bis 1982.

- Szenario 3 (bzw. 7)

Hier wurde der Trend der Ausgabenquote ohne Zinsen (bzw. Einnahmenquote) für 1970 bis 1982 gebildet und für die Jahre 1983 bis 1991 extrapoliert. Die Zeitreihe der Ausgabenquote setzt sich wiederum zusammen aus den tatsächlichen Quoten für die Jahre 1969 bis 1982 und den Quoten auf Basis der Extrapolation für 1983 bis 1991, wobei letztere im Gegensatz zum Szenario 1 (bzw. 5) das direkte Ergebnis der Extrapolation darstellen.

- Szenario 4 (bzw. 8)

Analog zu Szenario 3 (bzw. 7) wird ein Trend auf Basis der Entwicklung der Ausgabenquoten ohne Zinsen (bzw. Einnahmenquoten) gebildet, hier jedoch für die Jahre 1976 bis 1982.

Es läßt sich einwenden, daß nach 1982 eine Fortsetzung der bis dahin zu beobachtenden Trends nicht zwingend ist, ja gerade durch die Operation ' 82 die Weichen für eine Trendwende in der Haushaltspolitik der sozial-liberalen Koalition gestellt wurden. Zunächst soll jedoch die bis 1982 erfolgte Politik analysiert und bewertet werden. Die geplanten Maßnahmen finden in der anschließenden Beurteilung anhand der mittelfristigen Finanzplanung ihren Niederschlag.

Für das Wachstum und den rechnerischen Zinssatz wird zunächst die Annahme getroffen, daß bei der durch die Szenarien beschriebenen Entwicklung dennoch die in den Jahren nach 1982 tatsächlich realisierten Wachstumsraten bzw. Zinssätze verwirklicht worden wären. Dies ist nicht ganz unproblematisch, da sich die Haushaltspolitik der christlich-liberalen Koalition von der mit den Szenarien beschriebenen unterscheidet. Diese Annahme 


\section{Schaubild 8}

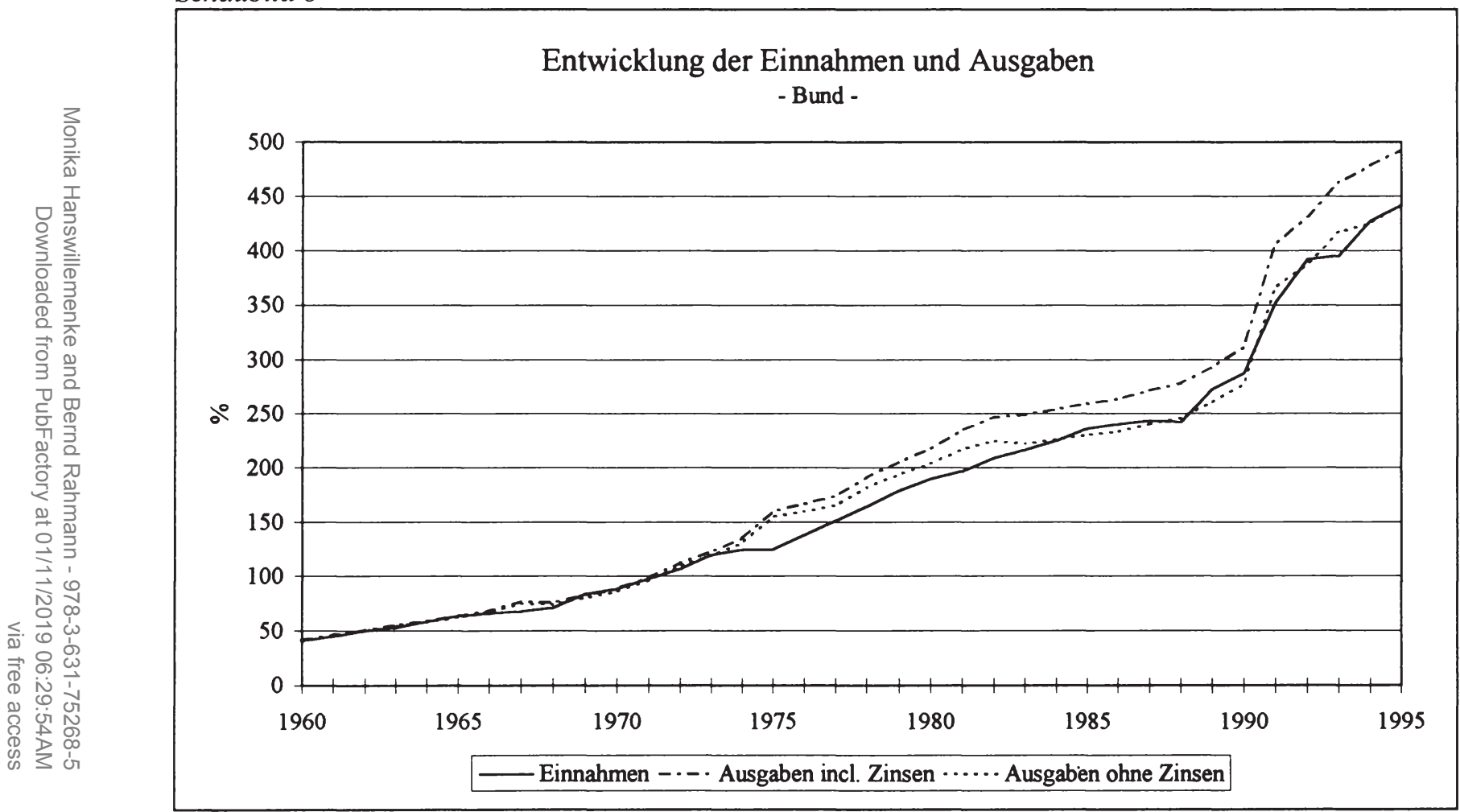




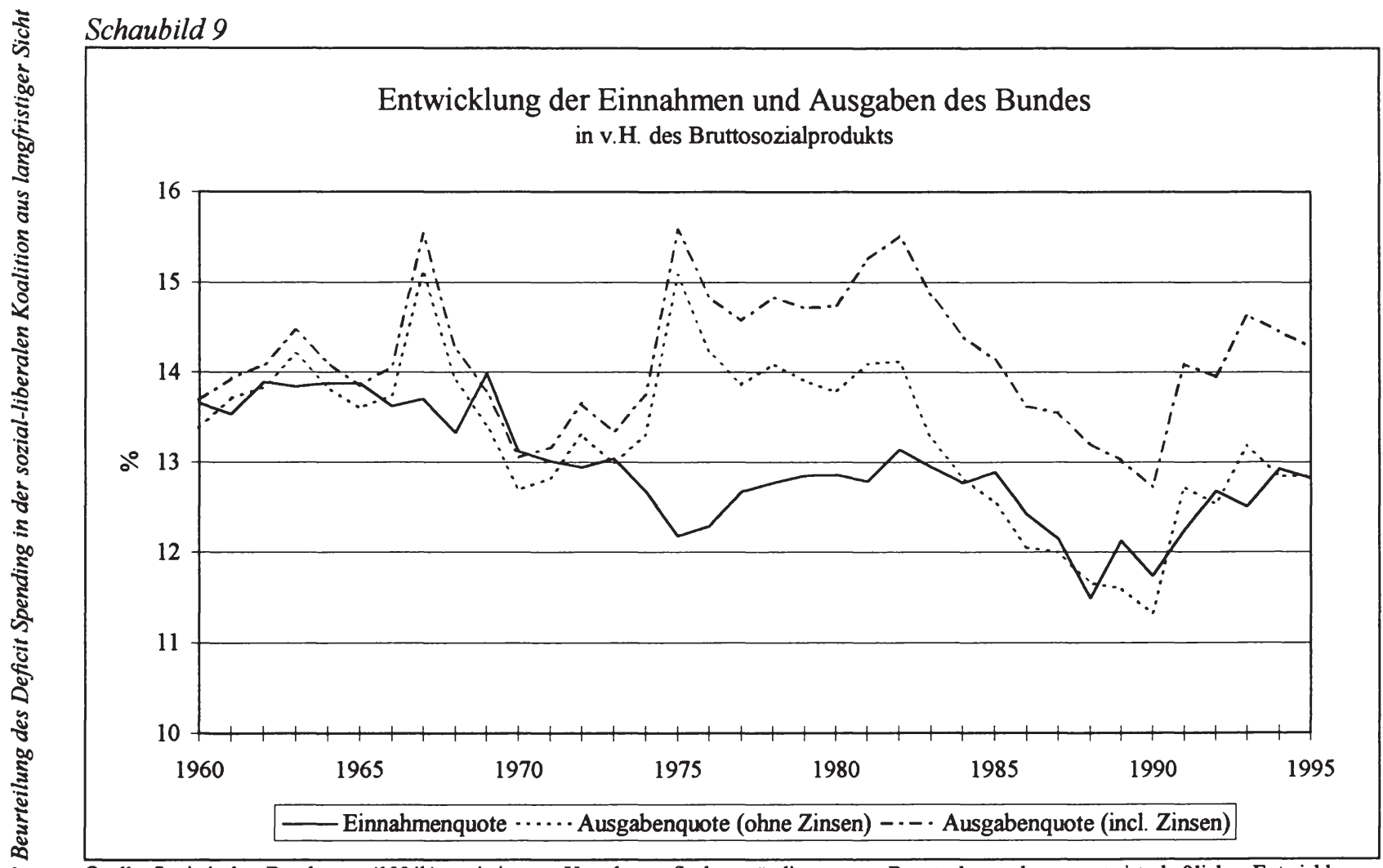

Quelle: Statistisches Bundesamt (1994b) sowie interne Unterlagen, Sachverstăndigenrat zur Begutachtung der gesamtwirtschaftlichen Entwicklung (1995); eigene Berechnungen. 
wird jedoch in Abschnitt 3.4 modifiziert. Da die vereinigungsbedingt hohen Wachstumsraten der Jahre 1990 und 1991 das Bild der langfristigen Indikatoren verfälschen würden, werden für die Jahre 1981 bzw. 1982 statt der 10jährigen Lücken 9- bzw. 8- jährige Lücken berechnet. Da das Jahr 1975 im Hinblick auf die Ausgaben- und Einnahmenentwicklung in gewisser Weise ein Ausnahmejahr darstellt und damit die grundsätzliche Ausgaben- und Einnahmenentwicklung verfälscht, wurde alternativ zu den Szenarien 1 (bzw. 5) und 3 (bzw. 7) ein Trend berechnet, in dem die Werte für 1975 durch Trendwerte der Jahre 1970 bis 1974 ersetzt wurden. Die auf Basis dieser bereinigten Trends berechneten Tragfähigkeitsindikatoren unterscheiden sich nur geringfügig von den Trends, die eine solche Korrektur nicht vornehmen. Im folgenden wird daher auf eine gesonderte Darstellung verzichtet.

\subsubsection{Die Entwicklung der Einnahmen - und Ausgabenlücken}

Die Einnahmen- und Ausgabenlücken auf Grundlage der oben beschriebenen Szenarien sind in Schaubild 10 bis 14 zusammengefaßt.

Die äußerst günstige Ausgangsposition zu Beginn der sozial-liberalen Regierungszeit mit außerordentlich hohen Wachstumsraten und einer gleichzeitig niedrigen Verzinsung der öffentlichen Schuld schlägt sich in negativen Werten der Indikatoren nieder. Der Wert der kurzfristigen Einnahmen- bzw. Ausgabenlücken für 1969 beträgt $-1,22 \%$ des Sozialprodukts. Das bedeutet, daß bei einer Erhöhung der Ausgaben und/oder einer Senkung der Steuern um einen Betrag, der insgesamt 1,22\% des Sozialprodukts entspricht, die Schuldenquote stabil geblieben wäre. Die mittel- bzw. langfristige Einnahmenlücke liegt bei $-1,47$ bzw. -0,65\%. Aus der Sicht des Jahres 1969 ist die Haushaltspolitik also tragfähig. Gemessen an den kurzfristigen Indikatoren bleibt sie dies auch bis 1973. Faktisch ist die Schuldenquote in dieser Zeit zurückgeführt worden (vgl. Abschnitt 3.1). Von Beginn an haben sich die Einnahmenlücken jedoch unter dem Aspekt der Tragfähigkeit verschlechtert. Den Einschnitt bildet das Jahr 1975 - rezessionsbedingt -, in dem dieser Indikator auf einen Wert von 3,11\% des Sozialprodukts hochschnellt. Durch die Konsolidierungspolitik seit Mitte der 70er Jahre wird der kurzfristige Indikator sukzessive auf $0,83 \%$ des Sozialprodukts zurückgeführt. Diese Ver- 


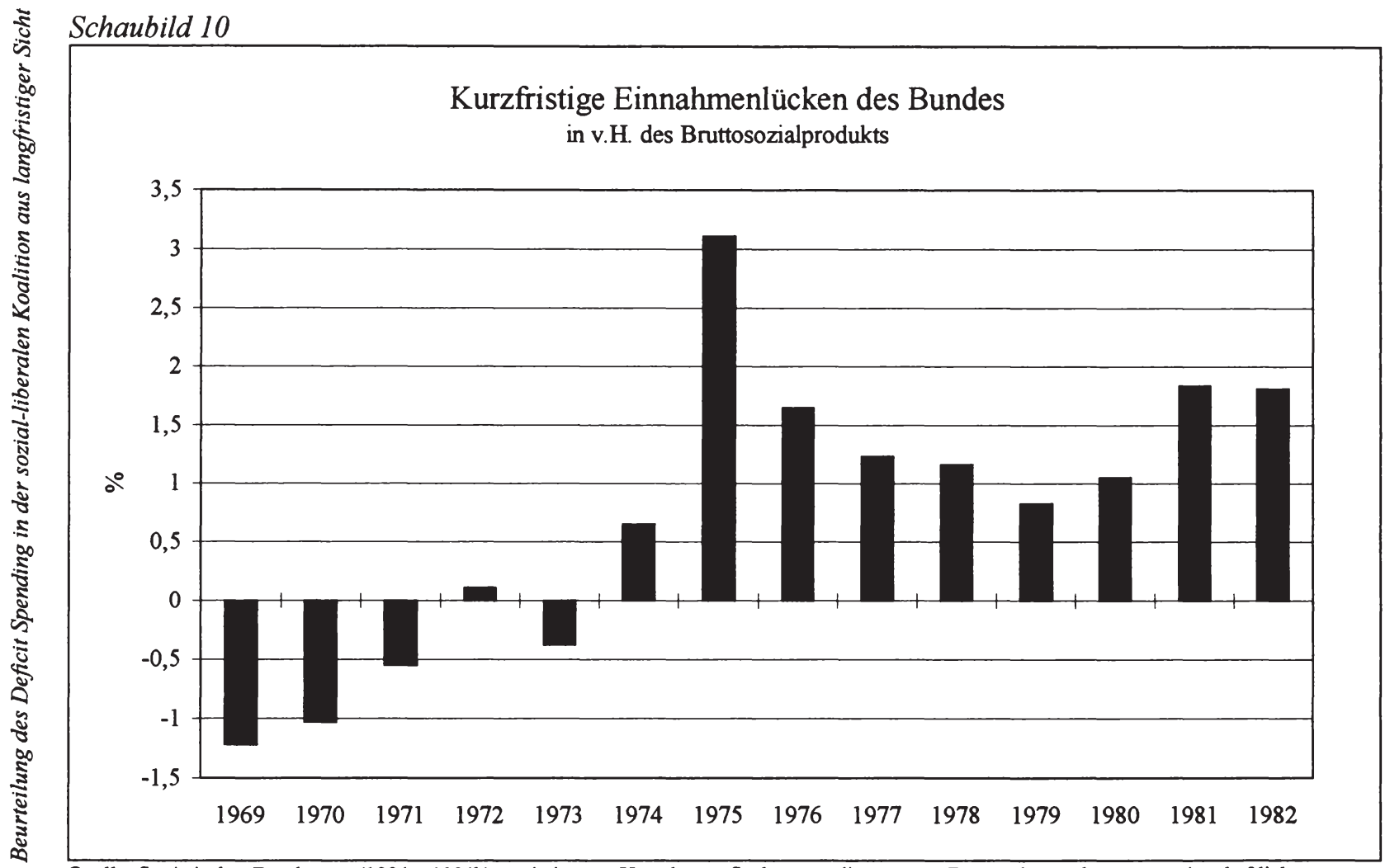

Quelle: Statistisches Bundesamt (1994a, 1994b) sowie interne Unterlagen, Sachverstăndigenrat zur Begutachtung der gesamtwirtschaftlichen Entwicklung (1995); eigene Berechnungen. 


\subsection{Zur Tragfähigkeit der Verschuldungspolitik}

besserung der Haushaltslage fand jedoch mit dem zweiten Ölpreisschock $(1979 / 80)$ ein vorläufiges Ende.

Die mittel- und langfristigen Indikatoren unterliegen weniger extremen Schwankungen. Durch die Berücksichtigung „zukünftiger“ Entwicklungen lassen sich eher Tendenzaussagen machen. Die beschriebene Verschlechterung der Verschuldungssituation schlägt sich mithin früher in den Indikatoren nieder. In diesem Falle interessanter ist jedoch die Abhängigkeit der Ergebnisse von der Wahl des Szenarios. Hier zeigt sich je nach Szenario eine mehr oder weniger klare Differenzierung. Die Ergebnisse der Szenarien 2 und 4 fallen besser aus als die der Szenarien 1 und 3, wobei sich die Unterschiede aber erst langfristig voll bemerkbar machen. Besonders deutlich ist die Differenz bei den Szenarien 3 und 4, die auf einer Extrapolation der Ausgabenquoten basieren. Das bedeutet, daß sich die von der sozial-liberalen Koalition angestrebte Konsolidierung in den Indikatoren für die Periode 1976 bis 1982 positiv niederschlägt. Hierin ist durchaus eine Wende in der sozial-liberalen Finanzpolitik Mitte der 70er Jahre erkennbar. Gleichwohl zeigt sich, daß die vorgenommenen Konsolidierungsanstrengungen noch nicht stark genug waren, um zu einer langfristig tragfähigen Verschuldungspolitik zu führen. So bewirkt die veränderte Haushaltspolitik eine Verbesserung der langfristigen Einnahmenlücke für 1982 um 0,71 Prozentpunkte (Vergleich von Szenario 3 und 4). Aber selbst im günstigsten Szenario 4 verbleibt eine Differenz zwischen notwendiger und tatsächlicher Einnahmenquote von 1,35 Prozentpunkten. Um die Schuldenquote 1982 bis $1989^{166}$ auf dem Niveau von 1981 zu halten, wäre eine entsprechende Reduzierung der Ausgaben oder Erhöhung der Einnahmen vonnöten gewesen. Bezogen auf den Haushalt 1982 hätte dies eine Senkung der Ausgaben um 8,7\% oder eine Erhöhung der Einnahmen bzw. Steuern um 10,3 bzw. 11,6\% bedeutet.

Die zugrundeliegende Konzeption läßt vermuten, daß der Verlauf der Einnahmen- und Ausgabenlücken im wesentlichen übereinstimmt. Aus kurzfristiger Sicht sind sie ohnehin identisch (vgl. Abschnitt 3.2.2). Aber auch mittel- und langfristig stimmen die Grundtendenzen überein. Auffallend ist jedoch die gegenläufige Entwicklung der beiden langfristigen Indikatoren in den Jahren 1969/70 sowie der stärkere Anstieg der mittel- und langfristigen

166 Auf 8 Jahre verkürzter Zeithorizont (s.o.). 
Mittelfristige Einnahmenlücken des Bundes

in v.H. des Bruttosozialprodukts

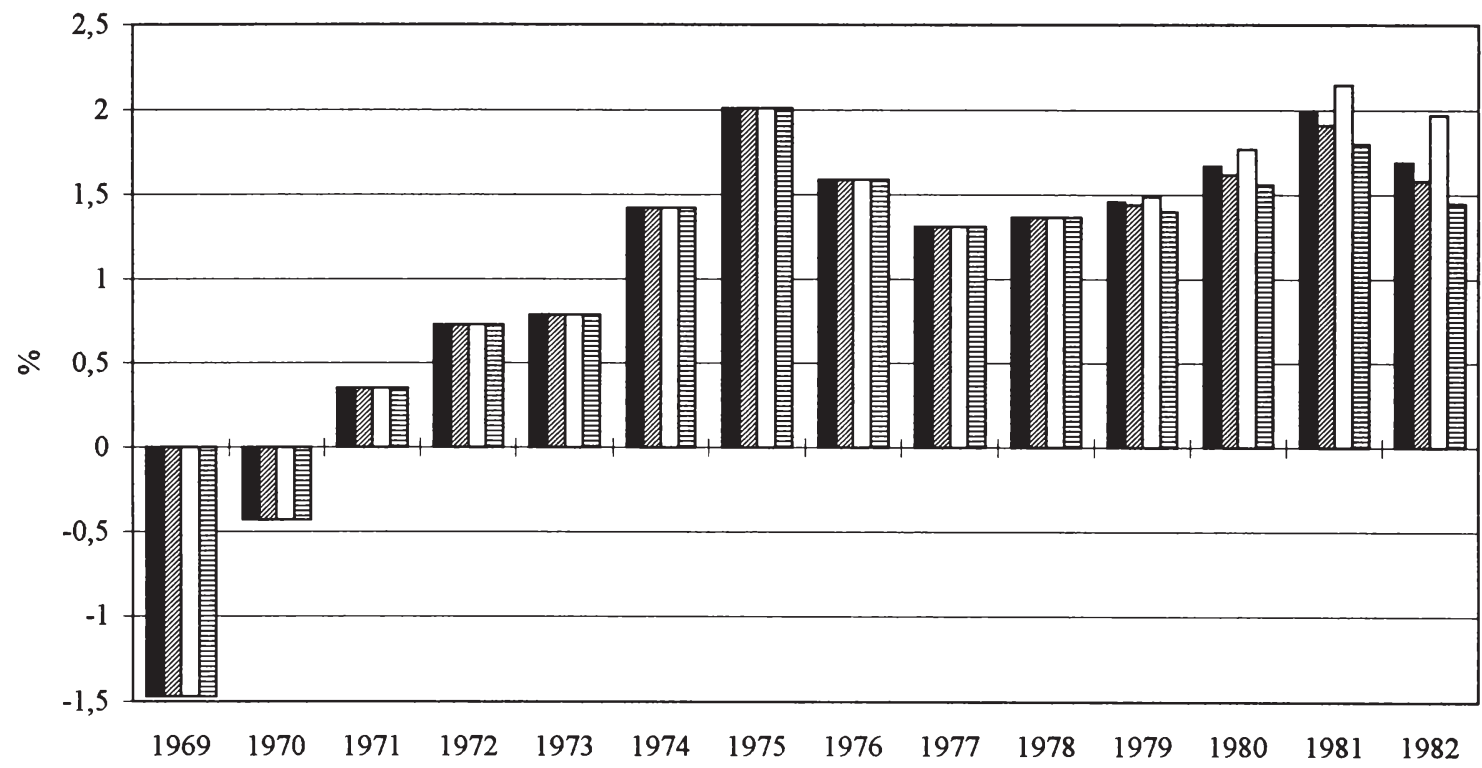

口 Szenario 1 Szenario $2 \square$ Szenario 3 目 Szenario 4

Quelle: Statistisches Bundesamt (1994a, 1994b) sowie interne Unterlagen, Sachverständigenrat zur Begutachtung der gesamtwirtschaftlichen Entwicklung (1995); eigene Berechnungen. 
Schaubild 12

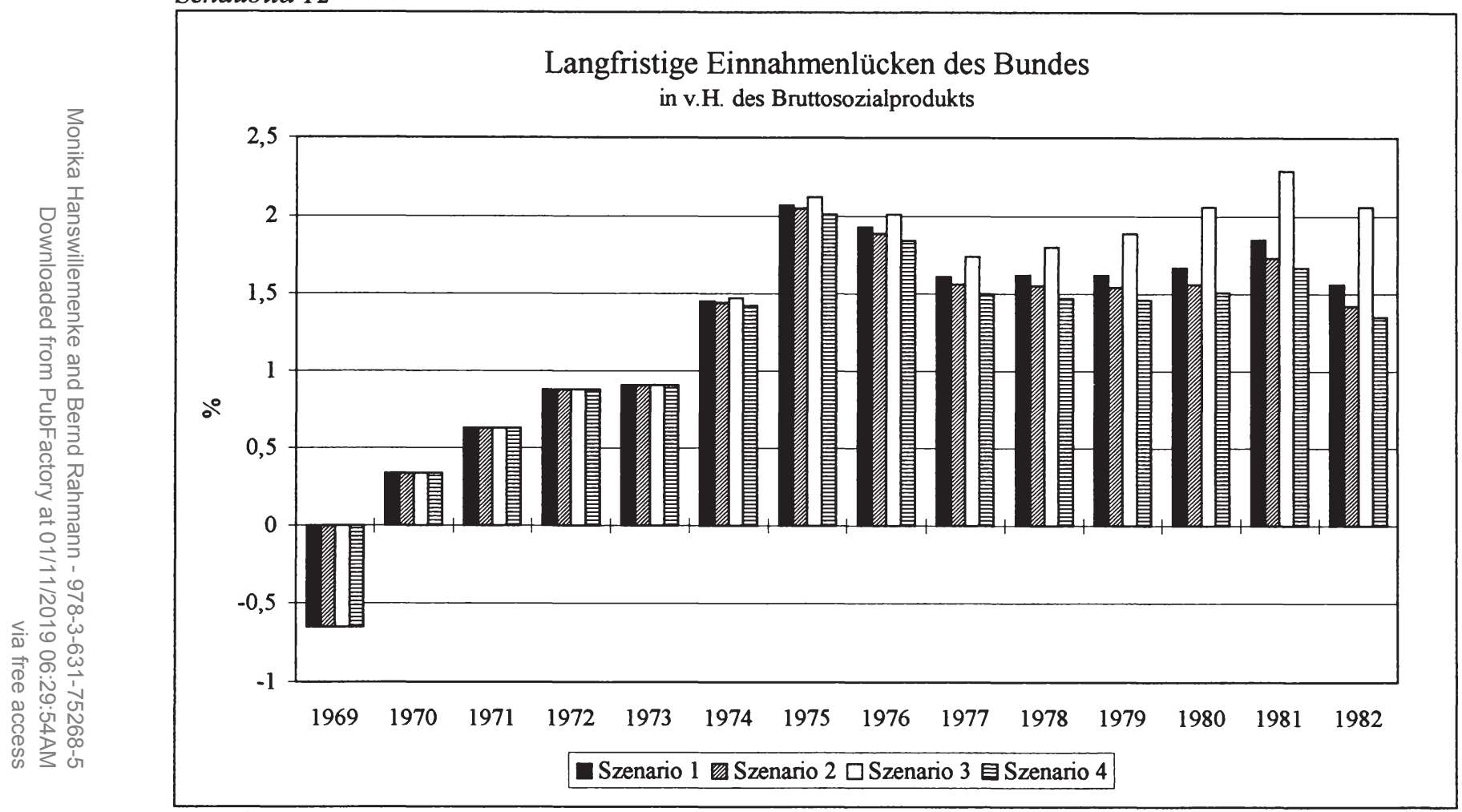


Ausgabenlücken gegenüber den Einnahmenlücken 1975. Während die Einnahmenquote des Bundes 1969 (die höchste des gesamten Betrachtungszeitraums) so hoch ist, daß dieser trotz des späteren Ausgabenwachstums eine negative Einnahmenlücke verzeichnet, führt die ,in der Zukunft“ durchschnittlich sinkende Einnahmenquote zusammen mit einer hohen Ausgabenquote $1969 \mathrm{zu}$ einer positiven Ausgabenlücke. Demgegenüber fallen die Ausgabenlücken 1970/71 dank erheblich niedrigerer Ausgabenquoten besser aus.

Insgesamt unterliegen die Ausgabenlücken mittel- und langfristig stärkeren Schwankungen als die Einnahmenlücken. Da letztere die Ausgaben mehrerer Jahre berücksichtigen, gleichen sich die stabilisierungsbedingten Schwankungen im Haushalt, die vorwiegend (zumindest bis Mitte der 70er Jahre) die Ausgabenseite betreffen, besser aus als bei den Ausgabenlücken, in die neben der zukünftigen Einnahmenentwicklung nur die Ausgabenquote des jeweiligen Jahres einfließt. Daran ändern auch die konjunkturbedingten Einnahmenschwankungen (eingebaute Stabilisatoren) nichts. Dies erklärt auch den stärkeren Anstieg der Ausgabenlücke im Rezessionsjahr 1975.

Mit wenigen Ausnahmen liegen die Ausgabenlücken sowohl mittel- als auch langfristig unterhalb der Einnahmenlücken. Dies ist auf ein tendenziell wachsendes Haushaltsvolumen (relativ zum Bruttosozialprodukt) zurückzuführen, d.h. auf ein Wachstum sowohl bei den Ausgaben als auch bei den ordentlichen Einnahmen. Denn um so stärker Ausgaben- und Einnahmenquote wachsen, desto höher sind die Einnahmenlücken und desto niedriger die Ausgabenlücken und desto größer ist die Differenz zwischen den Indikatoren. ${ }^{167}$ Ein Vergleich der verschiedenen Indikatoren wird jedoch in Abschnitt 3.3.4 vorgenommen, so daß auf eine nähere Betrachtung hier verzichtet wird.

167 Sinken sowohl Ausgaben- als auch Einnahmenquote tendenziell, dann liegen die Einnahmenlücken dagegen - ebenfalls nur tendenziell - unterhalb der Ausgabenlükken. Bei relativ zum Bruttosozialprodukt konstantem Haushaltsvolumen stimmen die beiden Indikatoren überein. 


\section{Schaubild 13}

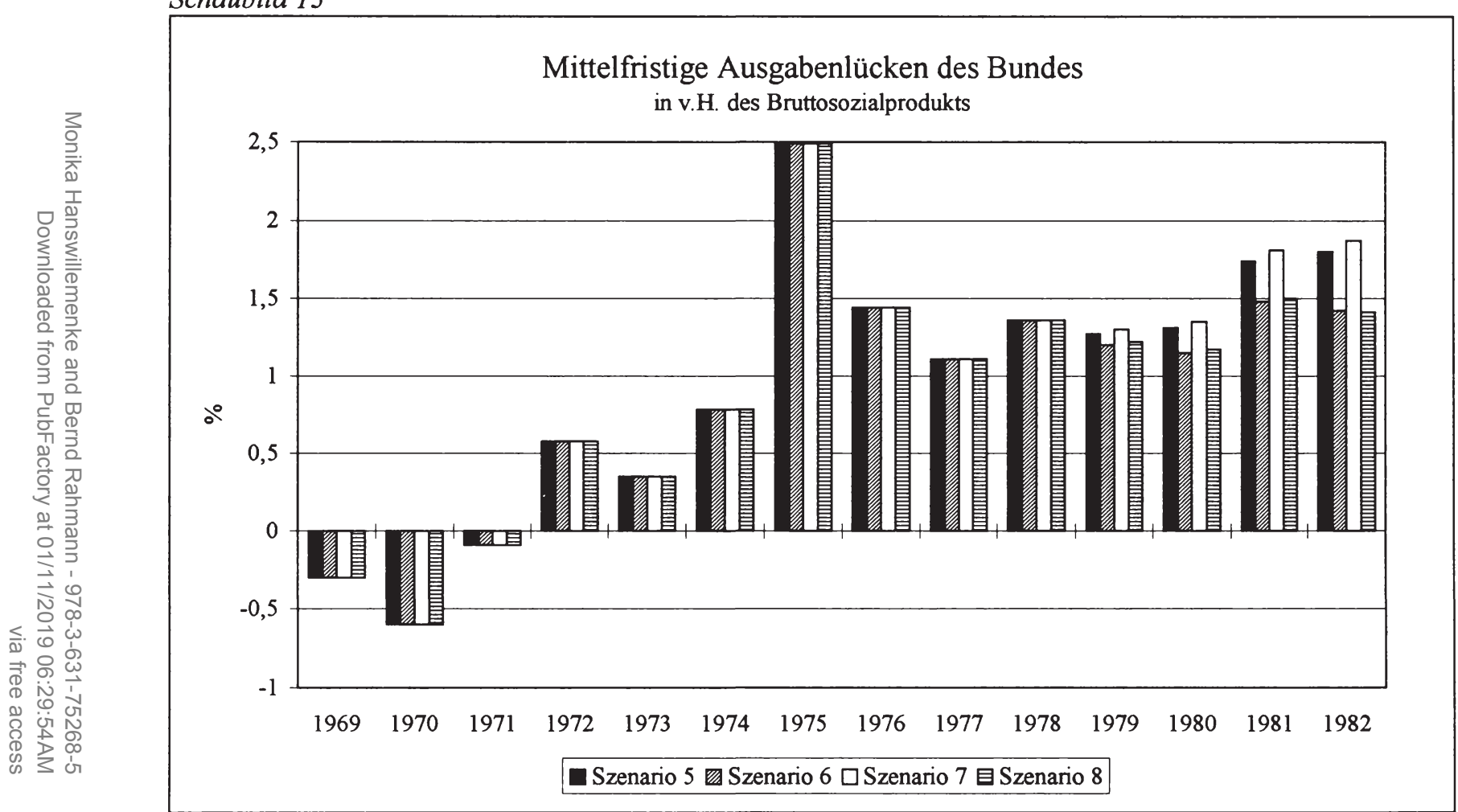




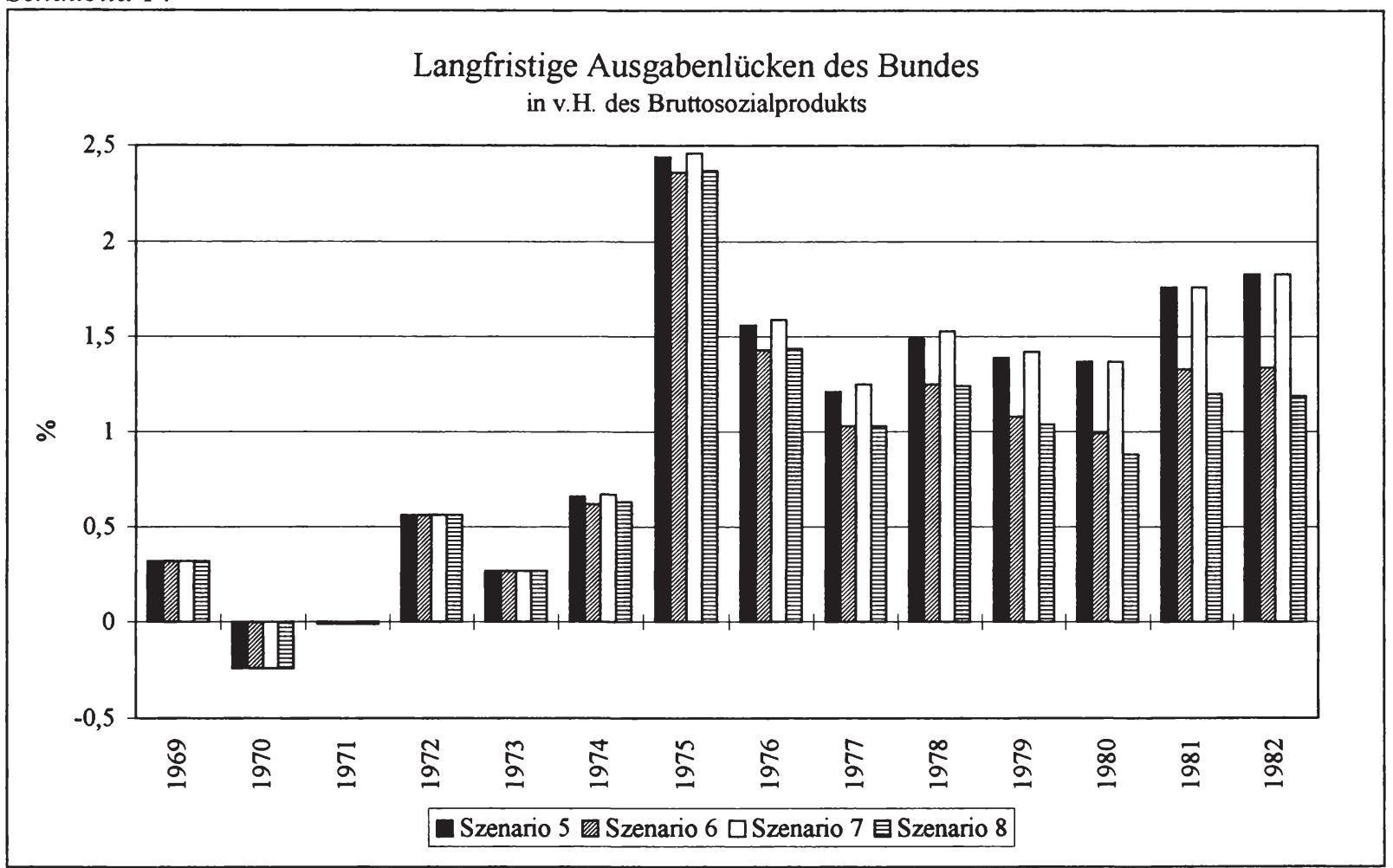

Quelle: Statistisches Bundesamt (1994a, 1994b) sowie interne Unterlagen, Sachverständigenrat zur Begutachtung der gesamtwirtschaftlichen Entwicklung (1995); eigene Berechnungen. 


\subsection{Zur Tragfahigkeit der Verschuldungspolitik}

\subsubsection{Die Verschuldungspolitik im Kontext vorhergehender und nachfolgender Jahre}

In Schaubild 15 ist die Entwicklung der Einnahmenlücken von Beginn der 60 er Jahre bis heute dargestellt. Anstelle der Szenarien wurde für die mittelund langfristigen Indikatoren jeweils die tatsächliche Ausgabenentwicklung herangezogen. Die Reihe der mittel- bzw. langfristigen Indikatoren kann folglich nur bis zum Jahre 1991 bzw. 1986 einschließlich berechnet werden. ${ }^{168}$ Die Werte für die Folgejahre sind damit nicht gleich Null, sondern die Reihe endet lediglich mit dem entsprechenden Jahr.

Die Schuldenpolitik der 60er Jahre kann allgemein als tragfähig angesehen werden. Zwar stieg im Rezessionsjahr 1967 die kurzfristige Lücke aufgrund der antizyklischen Politik auf $1,74 \%$ des Bruttosozialprodukts an, konnte jedoch schnell wieder abgebaut werden, so daß die mittel- und langfristigen Indikatoren auch weiterhin im negativen Bereich bleiben - und dies, obwohl der spätere Ausgabenanstieg zum Teil schon in die Werte mit einfließt.

Selbst im Vergleich mit den übrigen Jahren dieser Dekade war die Ausgangssituation der SPD/FDP-Koalition außerordentlich gut. Wie bereits in Abschnitt 3.3.2 beschrieben, haben sich die Einnahmenlücken im Verlauf der Regierungsperiode drastisch vergrößert. Über fast die gesamte Zeit muß die Haushaltspolitik als nicht tragfähig beurteilt werden. Verglichen mit den 60 er und 80er Jahren sowie der ersten Hälfte der 90er Jahre zeigt sich zudem, daß die Einnahmenlücken der 70er Jahre insgesamt die höchsten des gesamten Zeitraums sind. Aufgrund der von der späteren christlich-liberalen Koalition betriebenen Konsolidierungspolitik fallen die mittel-, vor allem aber die langfristigen Indikatoren besser aus als bei den oben beschriebenen Szenarien. Zu Beginn der 80er Jahre deuten die Indikatoren sogar eine langfristig tragfähige Politik an. Mit Blick auf diese Indikatoren läßt sich folgern, daß der CDU/FDP-Regierung in der Tat eine Wende gelungen ist. Politisch betrachtet war dies allerdings mit einer Höhergewichtung der langfristig angebotsorientierten Allokationseffizienz und einer Vernachlässigung des kurzfristigen Vollbeschäftigungsziels verbunden. Der Stabilitätsbegriff des

168 Natürlich könnten wiederum Trends gebildet und extrapoliert werden. Da es hier jedoch in erster Linie um eine Beurteilung der Politik der sozial-liberalen Koalition geht, soll hierauf verzichtet werden. 


\section{Entwicklung der Einnahmenlücken des Bundes}

in v.H. des Bruttosozialprodukts

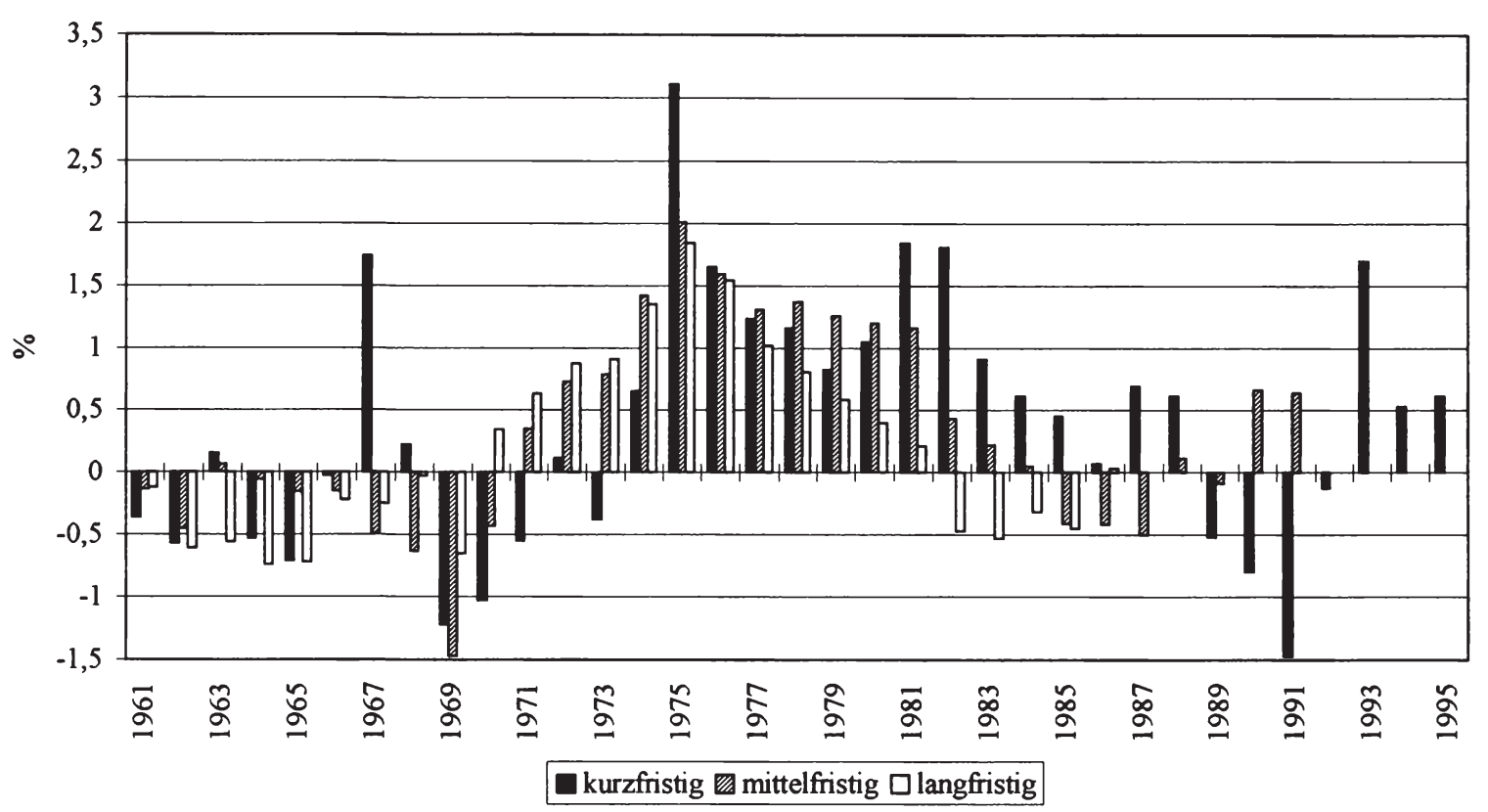

Anmerkung: Keine Angaben zu den mittelfristigen Werten der Jahre 1992 bis 1995 sowie den langfristigen Werten der Jahre 1986 bis 1995 (siehe Text).

Quelle: Statistisches Bundesamt (1994a, 1994b) sowie interne Unterlagen, Sachverständigenrat zur Begutachtung der gesamtwirtschaftlichen

Entwicklung (1995); eigene Berechnungen. 


\subsection{Zur Tragfahigkeit der Verschuldungspolitik}

$\S 1$ StabWG, der á priori gleichberechtigt Preisniveau, Beschäftigung, AuBenwirtschaft und Wachstum umfaßt, wird nunmehr radikal auf die Stabilität des Preisniveaus verkürzt. Anders als 1982 der sozial-liberalen Koalition hat sie dies bisher nicht die Regierungsmehrheit gekostet, obwohl Vollbeschäftigung bis heute nicht nur nicht erreicht wurde, sondern sich inzwischen auf einer Höhe befindet, die mit der Massenarbeitslosigkeit der Weltwirtschaftskrise Ende der 20er Jahre vergleichbar ist.

Neben der Haushaltskonsolidierung hat dann später nicht zuletzt der vereinigungsbedingte Boom 1990/91 Erfolge gebracht. Für die folgenden Jahre lassen sich ohne eine Ausgabenprognose allerdings keine langfristigen Werte mehr berechnen. Die Entwicklung der kurz- und mittelfristigen Einnahmenlücken deutet jedoch wieder eine Verschlechterung an. Die vereinigungsbedingten Aufwendungen haben erwartungsgemäß die Indikatoren in die Höhe getrieben. Dadurch, daß hierfür Sondervermögen außerhalb des Budgets gebildet wurden ${ }^{169}$, schlägt dieser Anteil jedoch weniger stark zu Buche, wie dies die Finanzpolitik der sozial-liberalen Koalition für sich gesehen während der 70er Jahre zu verzeichnen hat. ${ }^{170}$ Vergleichend muß festgestellt werden, daß seit Beginn der 90er Jahre ein erheblich wachsender Anteil der Verschuldung über Sondervermögen lief ${ }^{41}$, während der Anteil der Sondervermögen an der Gesamtverschuldung sowie auch ihr absoluter Schuldenstand im Laufe der 70er Jahre tendenziell gesunken ist.

169 Dies ist ein klarer Verstoß gegen den $\S 11 \mathrm{BHO}$, der die Haushaltsgrundsätze der Einheit und Vollständigkeit enthält, ohne die eine Vergleichbarkeit nicht bzw. nur erschwert möglich ist. Die Bildung dieser Sondervermögen ist so gesehen auch ein Politikum.

170 Die für die 80er Jahre beschriebene Entwicklung wird von Blanchard/Chouraqui/Hagemann/Sartor (1990) und Chouraqui/Hagemann/Sartor (1990) anhand kurzund mittelfristiger Indikatoren bestätigt, wenngleich die konkreten Werte sowohl voneinander als auch von den hier berechneten abweichen, letzteres u.a., weil eine unterschiedliche Abgrenzung des Haushalts vorgenommen wird.

171 Neben den ERP-Sondervermögen gehören hierzu seit 1990 der Fonds „Deutsche Einheit" und der Kreditabwicklungsfonds, dessen Schulden zusammen mit den Schulden der Treuhandanstalt seit 1995 vom Erblastentilgungsfonds übernommen wurden, seit 1994 das Bundeseisenbahnvermögen sowie seit 1995 der Ausgleichsfonds „Steinkohle“. 


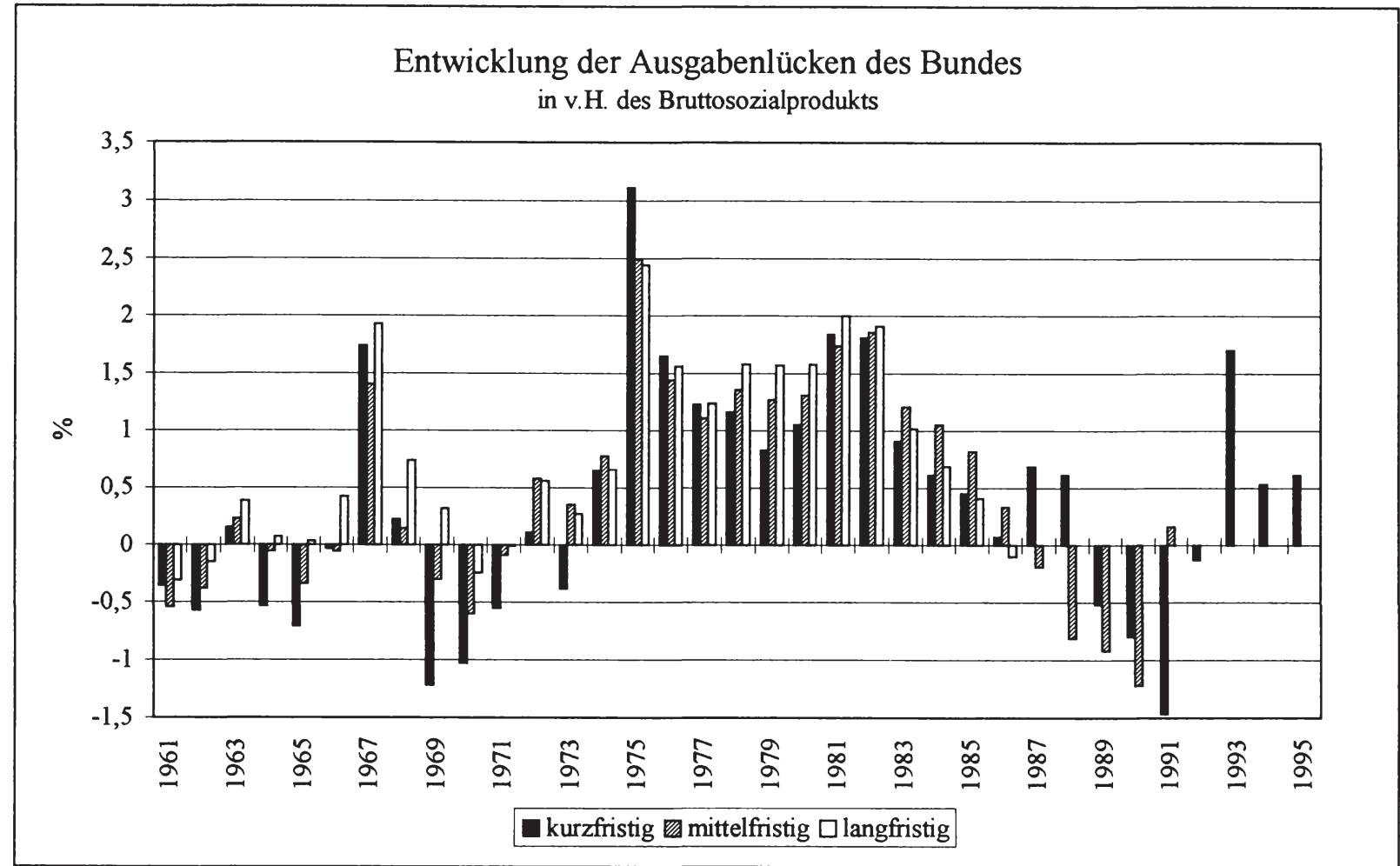

Anmerkung: Keine Angaben zu den mittelfristigen Werten der Jahre 1992 bis 1995 sowie den langfristigen Werten der Jahre 1986 bis 1995 (siehe Text).

Quelle: Statistisches Bundesamt (1994a, 1994b) sowie interne Unterlagen, Sachverständigenrat zur Begutachtung der gesamtwirtschaftlichen 
Die Ausgabenlücken bestätigen wiederum grundsätzlich die beschriebenen Ergebnisse, wobei auch hier - insbesondere bei den Langfristwerten - stärkere Schwankungen als bei den Einnahmenlücken festzustellen sind. Die mittel- und langfristigen Ausgabenlücken fallen vor allem in den Jahren 1975 bis 1982 insgesamt deutlich höher aus als die Einnahmenlücken und werden erst später auf ein tragfähiges $\mathrm{Ma} \beta$ reduziert. Hierfür ist zum einen die Verstärkung angebotsorientierter Politik in den Folgejahren mit ihren einnahmeseitigen Reduktionen, zum anderen sind die in diesem Zeitraum hohen und in den darauffolgenden Jahren sinkenden Ausgaben verantwortlich. ${ }^{172}$

Es sei jedoch nochmals darauf hingewiesen, da $\beta$ bei der Berechnung aller Indikatoren von einer Stabilisierung auf dem bereits erreichten Niveau ausgegangen wird. Auf die Konsequenzen der Festlegung eines konstanten Niveaus der Schuldenquote wird in Kapitel 4 eingegangen.

\subsubsection{Auswirkungen des Haushaltsgebarens auf die Entwicklung der Indikatoren}

In der Regel beschränken sich Tragfähigkeitsuntersuchungen auf die Berechnung einer Indikatorform, meist Einnahmen- oder Primärüberschußlükken. ${ }^{173}$ Grundsätzlich geben alle Indikatoren den quantitativen Handlungsbedarf zur Stabilisierung der Schuldenquote an. Bereits in den vorangegangenen Abschnitten wurde jedoch deutlich, daß sich je nach Haushaltsentwicklung durchaus Differenzen in den Ergebnissen ergeben. Diese sollen im folgenden dazu genutzt werden, zusätzliche Informationen bezüglich der sozial-liberalen Schuldenpolitik zu gewinnen.

Aus kurzfristiger Sicht ergibt sich zwischen Einnahmen-, Ausgaben- und Primärüberschußlücken bekanntlich kein Unterschied. In die mittel- und langfristigen einnahmen- bzw. ausgabenseitigen Lücken geht jedoch im Gegensatz zu den kurzfristigen Indikatoren sowie den Primärüberschußlük-

172 Ersteres führt zu einem Anstieg der (mittel- und langfristigen) Ausgabenlücken, letzteres zu einer Verschlechterung der Ausgaben- und einer Verbesserung der Einnahmenlücken.

173

Vgl. Blanchard (1990), Blanchard/Chouraqui/Hagemann/Sartor (1990), Chouraqui/ Hagemann/Sartor (1990) oder Institut ,Finanzen und Steuern“ (1993). 


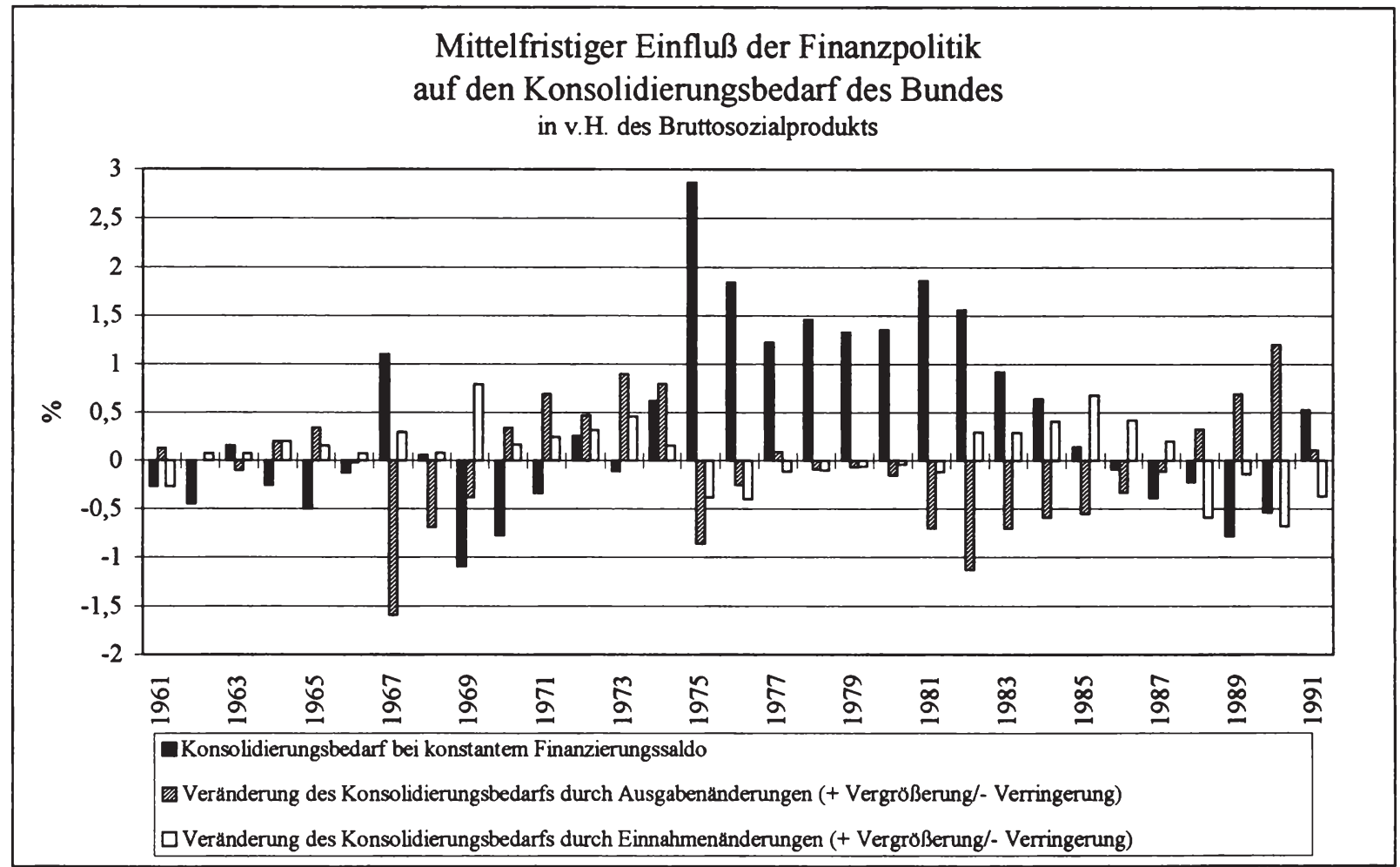

Quelle: Statistisches Bundesamt (1994a, 1994b) sowie interne Unterlagen, Sachverständigenrat zur Begutachtung der gesamtwirtschaftlichen Entwicklung (1995); eigene Berechnungen. 
ken die tatsächliche oder erwartete Entwicklung der Ausgaben bzw. Einnahmen ein. Im Falle einer konstanten Ausgaben- oder Einnahmenquote sind die Indikatoren wiederum identisch. Wächst oder fällt jedoch der Anteil der Ausgaben resp. Einnahmen am Sozialprodukt, dann differenziert sich das Ergebnis. Während Primärüberschußlücken somit lediglich den Status quo des Haushalts berücksichtigen, mithin neben dem aktuellen Primärdefizit bzw. -überschuß lediglich die Eigendynamik der Verschuldung widerspiegeln, fließen in die Einnahmen- und Ausgabenlücken auch die Änderungen in der Haushaltspolitik ein. Ein Vergleich der verschiedenen Lücken zeigt somit, in welche Richtung die Finanzpolitik Einfluß auf die Dynamik nimmt. Liegen die Einnahmenlücken (Ausgabenlücken) oberhalb der Primärüberschußlücken, dann hat die Haushaltspolitik ausgabenseitig (einnahmenseitig) die Dynamik verstärkt, im umgekehrten Falle hat sie sie abgeschwächt.

Für den Konsolidierungsbedarf bei konstantem Finanzierungssaldo, hinter dem die Primärüberschußlücken stehen, zeigt Schaubild 17 das von den anderen Indikatoren gewohnte Bild: Durch Primärüberschüsse oder im Vergleich zu späteren Jahren geringe Primärdefizite sowie begünstigt durch eine über dem Zinssatz liegende Wachstumsrate sind die Primärüberschußlücken der 60er Jahre eher gering, in den meisten Jahren sogar negativ. Hier bestätigt sich nochmals, daß im Falle negativer Zins-Wachstums-Differentiale ein Verschuldungsproblem nicht existiert bzw. bei Stabilisierung einer vorgegebenen (hier der aktuellen) Schuldenquote zumindest abgemildert wird. Eine nachhaltige Verschlechterung ergibt sich ab 1975, da nicht nur die Defizite zunehmen, sondern auch das Verhältnis von Wachstum und Verzinsung der öffentlichen Schuld ungünstiger wird. Für die späten $80 \mathrm{er}$ und frühen $90 \mathrm{er}$ Jahre gilt das bereits oben Gesagte: nach einer durchgreifenden Verringerung der Lücken zu Beginn der 80er Jahre ist in den letzten Jahren wieder ein vermehrter Konsolidierungsbedarf festzustellen.

Trotz der Übereinstimmung in der generellen Entwicklung der Indikatoren ergeben sich im einzelnen zum Teil beträchtliche Unterschiede. Die Änderungen im Konsolidierungsbedarf, die sich aus Wechseln im Haushaltsgebaren ergeben, sind für die tatsächlichen Haushaltsdaten in Schaubild 17 sowie für die Szenarien (die sich mittelfristig ab 1979 auswirken) in Diagramm 18 und 19 dargestellt. So liegen die mittelfristigen Ausgabenlücken von 1962 bis 1974 permanent oberhalb der entsprechenden Primärüberschußlücken, 


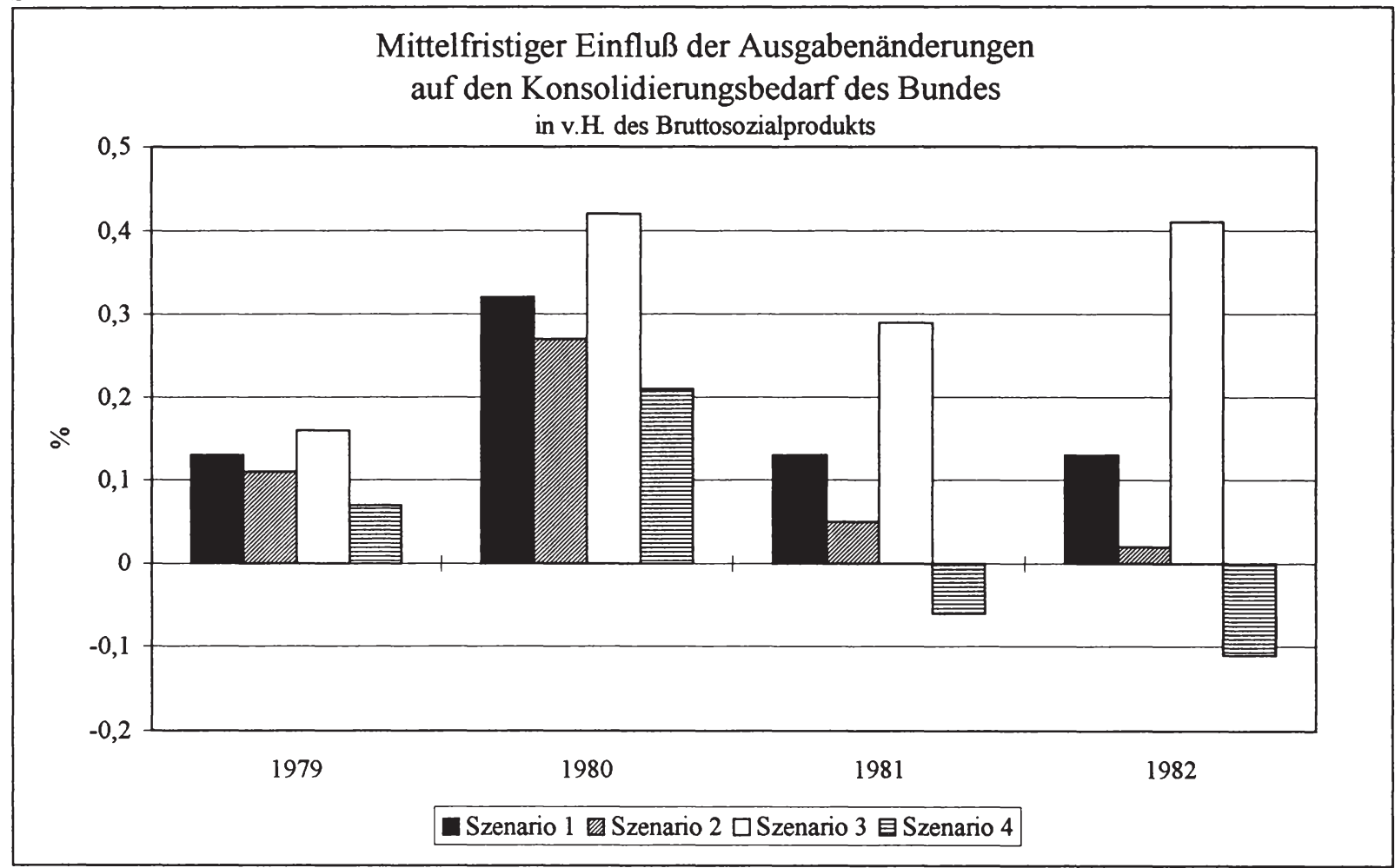

Quelle: Statistisches Bundesamt (1994a, 1994b) sowie interne Unterlagen, Sachverständigenrat zur Begutachtung der gesamtwirtschaftichen Entwicklung (1995); eigene Berechnungen. 


\section{Schaubild 19}

\section{Mittelfristiger Einfluß der Einnahmenänderungen}

auf den Konsolidierungsbedarf des Bundes

in v.H. des Bruttosozialprodukts

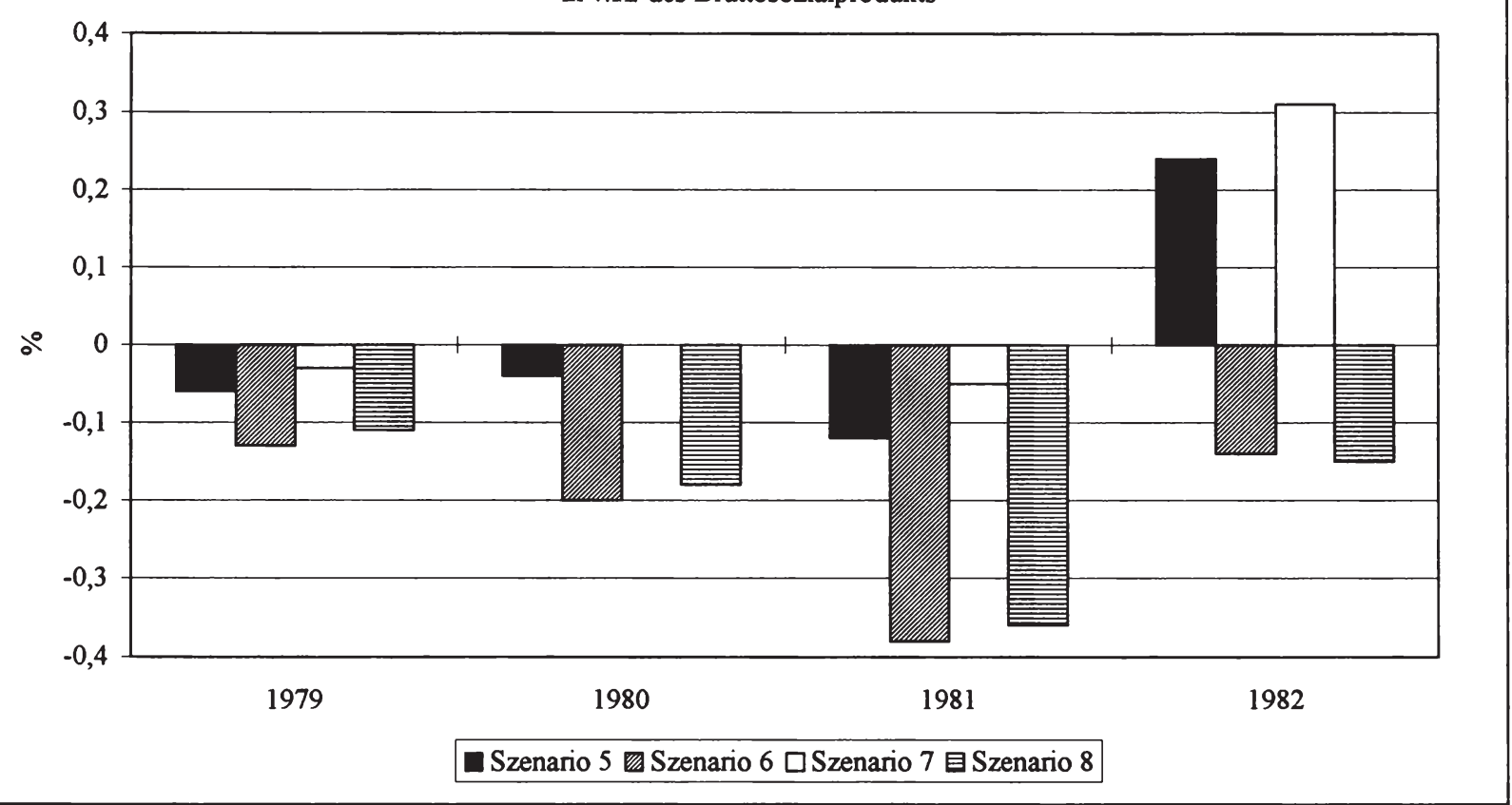


von 1975 bis 1981 ununterbrochen darunter, unabhängig von der Wahl des Szenarios. 1982 ist lediglich in Szenario 6 und 8 die Ausgabenlücke kleiner als die Primärüberschußlücke, in den anderen Szenarien ist sie größer. Hinter dieser Entwicklung steht eine zunächst sinkende und später tendenziell wieder steigende Einnahmenquote. Die Einnahmenseite hat also zunächst zu einer Vergrößerung des Konsolidierungsbedarfs gegenüber dem Status quo geführt, später jedoch zu einer Verminderung desselben beigetragen. Gegenüber dem Trend von 1970 bis 1982 ist für die Jahre 1976 bis 1982 eine Verbesserung von der Einnahmenseite des Haushalts her festzustellen.

Für die Einnahmenlücken ergibt sich kein gleichermaßen einheitliches Bild. Nachdem zu Beginn der 60er Jahre kein Muster zu erkennen ist, ergeben sich für die Differenz zwischen Einnahmen- und Primärüberschußlücken von 1966 an im 4-5-Jahres-Rhythmus wechselnde Vorzeichen: 1966 bis 1969 negativ, 1970 bis 1974 positiv, 1975 bis 1978 (mit Ausnahme von 1977) negativ, 1979 bis 1982 positiv (lediglich Szenario 4 weicht mit einem „leicht“ negativen Vorzeichen für 1981/82 davon ab).

Bis 1975 haben also relativ zum Sozialprodukt sinkende Einnahmen des Bundes - u.a. sinkende Steuereinnahmen - sukzessive zu einer Verschlechterung der Schuldenlage beigetragen, wobei dies in den Jahren 1966 bis 1969 durch ebenfalls sinkende Ausgaben (relativ zum Sozialprodukt) teilweise wieder (zum Teil auch mehr als) ausgeglichen wird. Zum Tragen kommt die negative Entwicklung der Einnahmen erst 1970, weil sich hier die kommenden Ausgabensteigerungen mittelfristig auszuwirken beginnen, wobei die Effekte der Ausgabenerhöhungen noch über die der Einnahmenminderungen hinausgehen. Ab 1975 tragen sowohl Ausgaben- als auch Einnahmenseite des Haushalts zu einer Verbesserung der Schuldensituation bei. Während die einnahmenseitigen Verbesserungen bis 1982 anhalten (zumindest für die Szenarien 6 und 8), sind ausgabenseitig ab 1979 wieder Verschlechterungen zu verzeichnen.

Relativ zur Höhe des Konsolidierungsbedarfs bei konstantem Finanzierungssaldo fallen die haushaltsbedingten Änderungen in der ersten Hälfte der Regierungszeit der sozial-liberalen Koalition wesentlich stärker aus als in der zweiten. Der sich durch die Haushaltsentwicklung ergebende zusätzliche Konsolidierungsbedarf geht zu Beginn der 70er Jahre sogar meist über den 


\subsection{Zur Tragfähigkeit der Verschuldungspolitik}

beim Status quo (unter Berücksichtigung der Zinsdynamik) vorhandenen hinaus. Zudem zeigt sich, daß die Ergebnisse für die Szenarien 2 und 4 bzw. 6 und 8 entschieden besser ausfallen als für die anderen Szenarien, was den Umschwung in der Haushaltspolitik seit Mitte der 70er Jahre nochmals bestätigt. Es zeigt sich aber auch, daß die Konsolidierung im wesentlichen auf der Einnahmenseite erfolgt. Lediglich für Szenario 4 zeigt sich in den Jahren 1981/82 ein Beitrag von Ausgabenkürzungen zur Stabilisierung der Schuldenquote, der jedoch gemessen an der nötigen Anpassung bescheiden ausfällt. Bei den langfristigen Indikatoren zeigt sich noch deutlicher, daß der Schwerpunkt der Schuldeneindämmung in den Jahren 1976 bis 1982 auf der Einnahmenseite lag. Dies steht im krassen Gegensatz zu der von der konservativ-liberalen Koalition befolgten Politik, bei der die Stabilisierung in den frühen $80 \mathrm{er}$ Jahren über die Ausgabenseite erfolgte, während die Einnahmenseite durch die angebotsorientierte Steuerpolitik einer Konsolidierung eher entgegenstand. Bei einer langfristigen Betrachtung kristallisieren sich die beschriebenen Ergebnisse zum Teil noch stärker heraus (vgl. Schaubild 20 und 21). Auf eine gesonderte Beschreibung soll jedoch verzichtet werden.

Es könnte vermutet werden, daß die einnahmenbedingten Verbesserungen der Tragfähigkeitsindikatoren gegen Ende der Regierungszeit in Steuererhöhungen begründet sind. Dies ist jedoch nicht der Fall. Die Steuerquote des Bundes (Steuern des Bundes in v.H. des Bruttosozialprodukts) ist zwar von 1976 auf 1977 konjunkturbedingt stark gestiegen, danach aber fast kontinuierlich gesunken und lag 1982 unterhalb des Wertes von 1976. Der steigende Trend in den Gesamteinnahmen läßt sich hingegen mit der Abführung des Bundesbankgewinns begründen (vgl. Abschnitt 3.5). Legte man nur die Entwicklung der Steuern für die Einnahmen zugrunde, d.h. bildete man einen Trend auf Basis der Steuerentwicklung unter Hinzurechnung der tatsächlichen sonstigen Einnahmen, dann hätte sich, wie gezeigt werden kann, die einnahmenseitige Verbesserung der Schuldensituation auf längere Sicht nicht eingestellt.

Weiterhin sollte angenommen werden, da $\beta$ bei einer konsequent angewandten antizyklischen Politik die Ausgabenquote über einen gesamten $\mathrm{Zy}$ klus konstant bleibt. Tatsächlich sind die um die Zinsen verminderten Gesamtausgaben (relativ zum Sozialprodukt) im Durchschnitt der Jahre 1970 


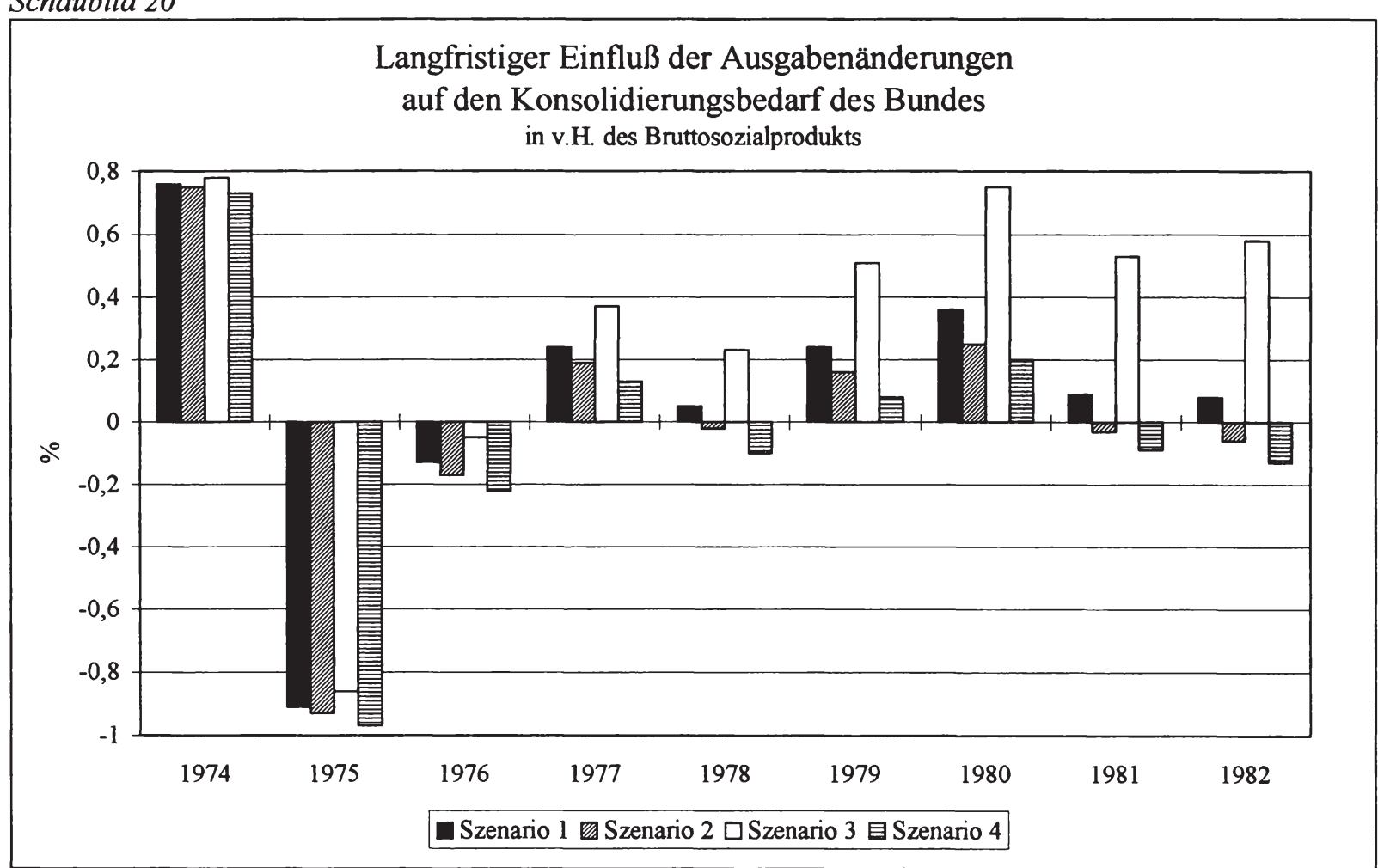

Quelle: Statistisches Bundesamt (1994a, 1994b) sowie interne Unterlagen, Sachverständigenrat zur Begutachtung der gesamtwirtschaftlichen Entwicklung (1995); eigene Berechnungen. 
Schaubild 21

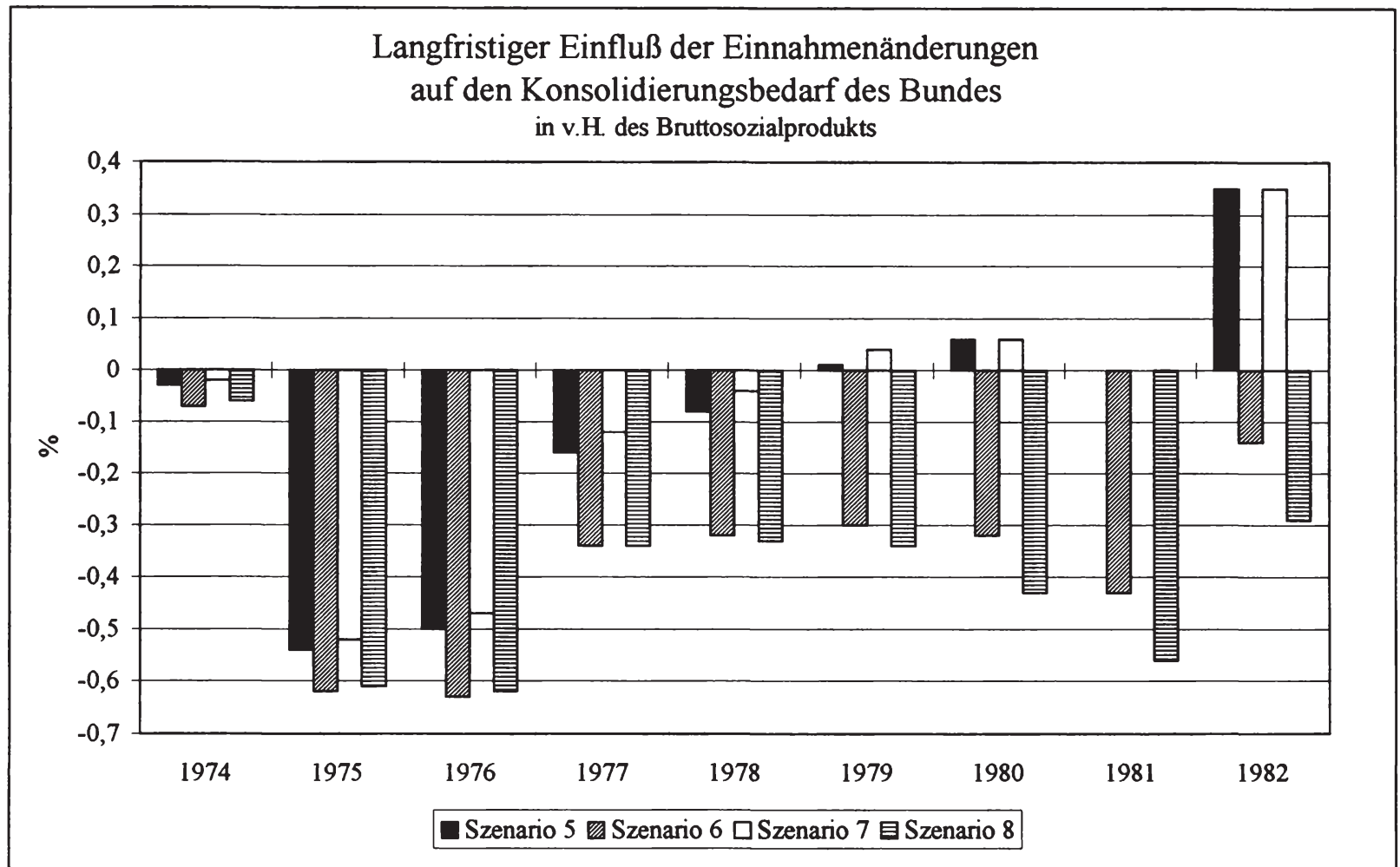


bis 1982 gegenüber der durchschnittlichen Quote für 1960 bis 1969 leicht gesunken. Der relative Anstieg der Ausgaben ist also allein auf die Zinsausgaben zurückzuführen. Bemerkenswert ist auch, daß die Ausgabenquoten 1967 und 1975 faktisch identisch sind, die Einnahmensituation hatte sich aber 1975 gegenüber 1967 derart verschlechtert, daß die Tragfähigkeitsindikatoren im Vergleich entschieden schlechter ausfallen. Aus Tragfähigkeitsüberlegungen bestätigt sich damit sowohl das euphorische Urteil über die Machbarkeit antizyklischer Stabilitätspolitik nach der Rezession 1967/68 wie auch die Frustration nach der Ölkrise.

\subsubsection{Tragfähigkeit der mittelfristigen Finanzplanung}

Nach $\S 9$ Abs. 1 des Stabilitäts- und Wachstumsgesetzes ist der Bund dazu verpflichtet, einen fünfjährigen Finanzplan aufzustellen, wobei das erste Planungsjahr mit dem laufenden Haushaltsjahr übereinstimmt ( $\S 50$ Abs. 2 Haushaltsgrundsätzegesetz). In ihm sollen die voraussichtlichen Ausgaben und deren Deckungsmöglichkeiten dargestellt werden, und zwar ,in ihren Wechselbeziehungen zu der mutmaßlichen Entwicklung des gesamtwirtschaftlichen Leistungsvermögens“. Die mittelfristige Finanzplanung sollte also mehr darstellen als eine einfache Extrapolation der jeweils aktuellen Situation. Von der Bundesregierung ${ }^{174}$ sowie auch von den meisten Autoren $^{175}$ wurde die mittelfristige Finanzplanung charakterisiert als „ein in Zahlen gekleidetes Regierungsprogramm, in dem die zeitlichen Prioritäten und die positiven und negativen Schwerpunkte sichtbar gemacht werden“. Konjunkturelle Schwankungen werden innerhalb des Fünfjahreszeitraums nicht mit einbezogen. Der mittelfristige Finanzplan sollte dagegen die Entwicklung darstellen, die - nach erfolgter Stabilisierung - im Mittel angestrebt wurde. ${ }^{176}$ Mit seiner mittelfristigen Ausrichtung ist der Finanzplan daher ge-

174 Vgl. Bundesministerium der Finanzen (1967), Finanzbericht 1968, S. 106 sowie Brandt (1969), S. 1123.

176 Auf die Probleme der mittelfristigen Finanzplanung kann hier nicht näher eingegangen werden. Ende der 60er bis etwa Mitte der 70er Jahre wurden diese eingehend diskutiert. Vgl. hierzu z.B. Fischer-Menshausen (1968), Gresser (1974), Grund (1968), Klementa (1972), Nachtkamp (1976), Schlecht (1968), Schlömann (1972), Schmidt/Wille (1970) oder Zunker (1972). 
eignet, die finanzpolitischen Planungen der Bundesregierung auf ihre Tragfähigkeit hin zu überprüfen. Zusätzlich zu der Berechnung auf Grundlage der Szenarien sind daher die Tragfähigkeitsindikatoren auf Basis der mittelfristigen Finanzplanung kalkuliert worden.

Um die Wechselwirkungen zwischen Haushalt und gesamtwirtschaftlicher Entwicklung erkennbar zu machen, wurden mittelfristige Zielprojektionen erstellt; diese basieren auf einem geschlossenen volkswirtschaftlichen Kreislaufmodell, in das Schätzungen über die voraussichtliche Entwicklung der Produktionsfaktoren und ihr Verhältnis zueinander sowie zum Produktionsergebnis einflossen. Die Schätzungen der Zielprojektionen des Wachstums, der Beschäftigung, der Preisniveaustabilität und des Außenbeitrags unterliegen jedoch recht willkürlichen Annahmen, so daß sie letztlich eher Wunschprojektionen als realistische Schätzungen der Zukunft (soweit dies überhaupt möglich ist) darstellen. Die Zugrundelegung projektierter Daten ist insofern mit dem Finanzplan vereinbar, als er - zumindest anfänglich - auch als ein Instrument zur Verwirklichung einer erwünschten Entwicklung angesehen wurde und nicht als reines Prognoseinstrument. ${ }^{177}$ Es ist daher auch vertretbar, neben den Ausgaben und Einnahmen des Finanzplans die WachstumsProjektionen in die Tragfähigkeitsanalyse mit einfließen zu lassen. Darüber hinaus werden - wie bereits in den vorhergehenden Abschnitten - die Schuldendaten des Statistischen Bundesamtes zugrundegelegt. Von den Wachstumsprognosen wurde für die Jahre 1979 bis 1982, in denen jeweils eine Bandbreite für das projektierte Bruttosozialprodukt angegeben ist, der jeweils pessimistischste Wert gewählt. Die Ergebnisse sind in Schaubild 22 dargestellt.

Bis 1973/74 - und damit länger als bei den Indikatoren auf Basis der dann mittelfristig tatsächlich realisierten Werte - zeigt sich eine tragfähige Politik. Für 1975 erfolgt dann ein abrupter Anstieg für alle Indikatoren. Die Einnahmenlücke kann in den Folgejahren - mit zwischenzeitlichen Verschlechterungen 1978 und 1981 - wieder auf ihr tragfähiges Niveau von 1974 zurückgeführt werden. Ausgabenlücke und Primärüberschußlücke werden ebenfalls abgebaut, bleiben indessen auf hohem Niveau. Die Ausgabenlücke fällt bei-

177 Zu den Schätzungen der Zielprojektionen vgl. etwa Schmidt/Wille (1970), S. 20-28 sowie die dort angegebene Literatur. Eine ausführlichere Analyse liefert Nachtkamp (1976), insbesondere S. 49-81. 


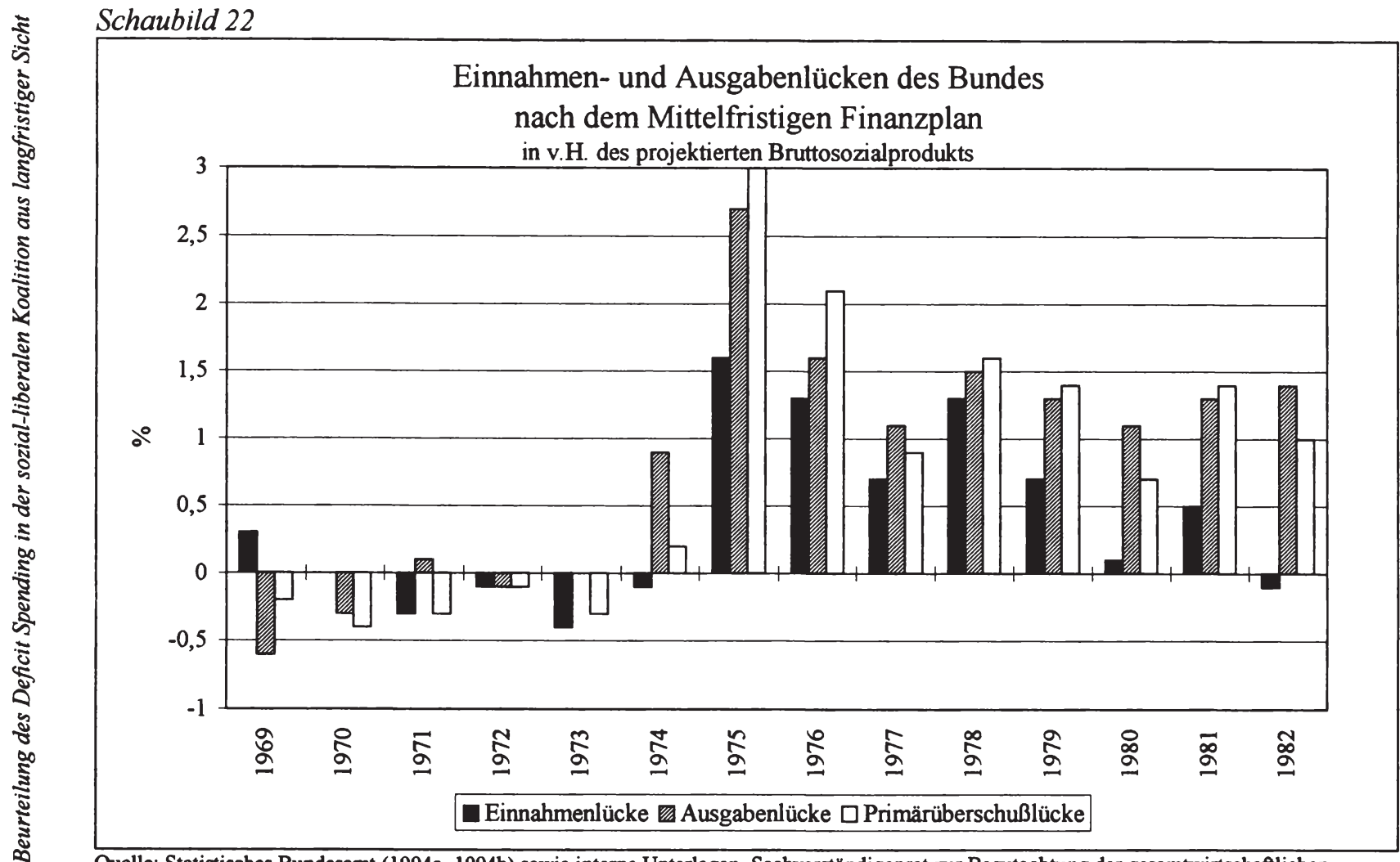

Quelle: Statistisches Bundesamt (1994a, 1994b) sowie interne Unterlagen, Sachverstăndigenrat zur Begutachtung der gesamtwirtschaftlichen Entwicklung (1995); eigene Berechnungen. 
spielsweise nicht unter einen Wert von 1\% des (projektierten) Bruttosozialprodukts zurück.

Ein Vergleich der Einnahmenlücken auf Basis der mehrjährigen Finanzplanung mit denen auf der Grundlage der tatsächlichen bzw. der Szenario-Werte zeigt, daß die Werte der Finanzplanung in den meisten Jahren besser ausfallen. In den besonders interessanten Jahren nach 1975 zeigen die Finanzpläne einen eindeutigen Trend hin zur Tragfähigkeit, während eine solche Entwicklung in den Szenarien lediglich angedeutet wird und die Finanzpolitik faktisch weit davon entfernt war, tragfähig zu sein.

Bei einem ebensolchen Vergleich für die Ausgabenlücken stellt sich heraus, daß - insbesondere in den Jahren 1973 bis 1982 - die Ausgabenlücken die tatsächliche Entwicklung besser wiedergeben als die Einnahmenlücken. So verzeichnen auch beide 1981/82 einen Anstieg. Dies bedeutet aber auch, daß die Haushaltspolitik aufgrund der Finanzplanung anhand dieser Indikatoren als nicht tragfähig beurteilt werden muß.

Bis auf die Jahre 1969 und 1970 liegt die Einnahmenlücke stets unterhalb der Ausgabenlücke und unterhalb bzw. (für die Jahre 1971/72) auf gleicher Höhe mit der Primärüberschlußlücke. Die Ausgabenlücke wiederum liegt 1969 unterhalb, in den Jahren 1970 bis 1974 oberhalb der Primärüberschußlücke, danach mit Ausnahme der Jahre 1977, 1980 und 1982 darunter. Zu Beginn der 70er Jahre sind es also hauptsächlich die mittelfristig geplanten Einnahmen, die zu einer Verschlechterung der - insgesamt aber tragfähigen - Werte führen, während die geplanten Ausgaben teilweise sogar zu einer Verbesserung der Tragfähigkeit beitragen. Ab Mitte der 70er Jahre sind es ebenfalls die Ausgaben, die nach Finanzplan zu einer Verringerung der Schuldendynamik beitragen. Es erfolgt zwar auch eine Konsolidierung auf der Einnahmenseite des Haushalts, ihr Effekt ist hierbei aber im Vergleich zur Ausgabenseite eher gering. In den Jahren 1980 und 1982 trägt die Einnahmenpolitik sogar deutlich zu einer Verstärkung der vom Status quo beeinflußten Dynamik bei.

Die mittelfristige Planung steht damit zum Teil im Gegensatz zur realisierten Politik, bei der es überwiegend die Ausgaben sind, die zu einer Verschlechterung der Schuldensituation beitragen, sowohl Anfang der 70er Jahre als 
auch zum Ende. In Szenario 4 wurde zwar eine gewisse Verbesserung festgestellt, die jedoch bei weitem nicht an die Plandaten heranreicht. Bei den Einnahmen muß differenziert werden zwischen der Entwicklung der Steuern und der sonstigen Einnahmen. Wird dem Verlauf der Gesamteinnahmen der Trend der Steuern zugrundegelegt, dann kann die im Finanzplan anvisierte Einnahmenpolitik als tendenziell verwirklicht angesehen werden. Die oben beschriebene Umorientierung der sozial-liberalen Wirtschaftspolitik hin zu einer Angebotsorientierung schlägt sich somit in der mittelfristigen Finanzplanung nieder und findet sich bei der Realisierung erwartungsgemäß auf der Einnahmenseite des Haushalts wieder.

Die Dynamik der Verschuldung selbst wird im Finanzplan 1969 bis 1973 sowie 1970 bis 1974 und 1971 bis 1975 überschätzt, da mit einem geringeren Sozialproduktswachstum gerechnet wurde. In den folgenden Jahren liegt das tatsächliche Bruttosozialprodukt bzw. Wachstum stets unterhalb der Zielprojektionen, so daß hier die Eigendynamik stets unterbewertet ist. Bei Zugrundelegung des tatsächlichen Bruttosozialprodukts sowie der tatsächlichen Wachstumsraten fallen die Primärüberschußlücken mithin zunächst günstiger und später schlechter aus als beim projektierten Bruttosozialprodukt.

Insgesamt läßt sich feststellen, daß die sich in der mittelfristigen Finanzplanung niederschlagende Ausgabenpolitik gegen Ende der sozial-liberalen Regierungszeit tragfähig ist - im Gegensatz zu dem, was die Kritik an der Politik vermuten läßt -, nicht jedoch die Einnahmenpolitik, was sich zwangsläufig aus der stärkeren Angebotsorientierung ergibt. Auf einen Vergleich zwischen mittelfristiger Finanzplanung und Realisation anhand konkreter Werte wurde hingegen verzichtet, da den Berechnungen unterschiedliche Datenbasen zugrunde liegen.

\subsubsection{Die Haushaltspolitik des Bundes im Rahmen des Gesamthaushalts}

Nach $\S 14$ des Stabilitäts- und Wachstumsgesetzes gelten wesentliche Paragraphen sinngemäß für die Haushalte der Länder - namentlich die $\S \S 5,6$ Abs. 1 und $2, \S \S 7,9$ bis 11 sowie $\S 12$ Abs. 1 . Gemeinden und Gemeindeverbände haben ebenfalls den Zielen des $\S 1$ des Stabilitäts- und Wachs- 


\subsection{Zur Tragfähigkeit der Verschuldungspolitik}

tumsgesetzes Rechnung zu tragen ( $§ 16$ Abs. 1), wobei die Länder „durch geeignete Maßnahmen darauf hinzuwirken“ haben, „daß die Haushaltswirtschaft der Gemeinden und Gemeindeverbände den konjunkturpolitischen Erfordernissen entspricht" ( $\$ 16$ Abs. 2). Tatsächlich verhielten sich die Gemeinden - nicht zuletzt wegen ihrer restringierten Verschuldungsmöglichkeiten - eher prozyklisch. Lediglich Bund und Länder - aber auch diese mit Einschränkungen - trugen dem Stabilitäts- und Wachstumsgesetz durch ein antizyklisches Verhalten Rechnung. ${ }^{178}$

Die Unterschiede im Haushaltsgebaren müssen sich naturgemäß in der Schuldensituation der Gebietskörperschaften niederschlagen. Um zu untersuchen, wie sich die Tragfähigkeit bzw. Nicht-Tragfähigkeit des Bundes auf den Gesamthaushalt auswirkt und inwieweit die Entwicklung des Bundes durch die der Länder und Gemeinden kompensiert oder prononciert wird, wurden die diversen Schuldenindikatoren auch für den Gesamthaushalt sowie Länder und Gemeinden/Gemeindeverbände hergeleitet. Der Gesamthaushalt in der hier vorgenommenen Abgrenzung umfaßt neben den Gebietskörperschaften - Bund, Länder und Gemeinden - auch die Sondervermögen. Analog zum Bund werden für den Gesamthaushalt verschiedene Szenarien gebildet. Zwar lagen beim Bund sowie den einzelnen Ländern und Gemeinden unterschiedliche politische und ökonomische Konstellationen vor; um jedoch festzustellen, ob sich die Grundausrichtung und damit die Schuldensituation des Gesamthaushalts grundlegend von der des Bundes unterscheidet, läßt sich diese Vorgehensweise rechtfertigen. ${ }^{179}$ Bei der Analyse der

178 Zum Verhalten der Gebietskörperschaften im Konjunkturverlauf vgl. z.B. Elsner (1978) oder Kock (1975).

179

Für Länder und Gemeinden wurde auf eine Szenario-Bildung aus den genannten Gründen verzichtet. Zwischen den Bundesländern bestand über die Einbindung in die Stabilisierungspolitik noch am ehesten ein Zusammenhang der Haushaltspolitiken. Bei den Gemeinden kann jedoch kaum von einer einheitlichen Grundrichtung ausgegangen werden, und auch der Zusammenhang zur Bundespolitik ist allenfalls höchst indirekt, so daß eine Trendbildung inhaltlich nicht begründet werden kann. Gleichwohl werden die verschiedenen Indikatoren für die aggregierten Länder und Gemeinden sowie den Gesamthaushalt auf der Basis der tatsächlichen Haushaltsdaten berechnet. Allerdings werden die Ergebnisse nicht gesondert aufgeführt, da Länder und Gemeinden nicht Gegenstand dieser Studie sind. Die Resultate werden lediglich zur Erklärung der Unterschiede beim Bund und beim Gesamthaushalt verwendet. 
Schuldenentwicklung wird zunächst auf die Ergebnisse auf Basis der Primärüberschußlücken eingegangen, um dann den Einfluß von Ausgaben und Einnahmen näher zu spezifizieren.

Tabelle 3: Primärüberschußlücken - Vergleich Bund und Gesamthaushalt

\begin{tabular}{|c|c|c|c|c|c|c|}
\hline \multirow{3}{*}{ Jahr } & \multicolumn{6}{|c|}{ in v.H. des Bruttosozialprodukts } \\
\cline { 2 - 7 } & \multicolumn{2}{|c|}{ kurzfristig } & \multicolumn{2}{c|}{ mittelfristig } & \multicolumn{2}{c|}{ langfristig } \\
\cline { 2 - 7 } & Bund & $\begin{array}{c}\text { Gesamt- } \\
\text { haushalt }\end{array}$ & Bund & $\begin{array}{c}\text { Gesamt- } \\
\text { haushalt }\end{array}$ & Bund & $\begin{array}{c}\text { Gesamt- } \\
\text { haushalt }\end{array}$ \\
\hline 1969 & $-1,22$ & $-2,81$ & $-1,09$ & $-2,54$ & $-0,82$ & $-1,96$ \\
1970 & $-1,03$ & $-1,15$ & $-0,77$ & $-0,53$ & $-0,62$ & $-0,18$ \\
1971 & $-0,55$ & 0,23 & $-0,34$ & 0,73 & $-0,29$ & 0,84 \\
1972 & 0,11 & 0,07 & 0,26 & 0,44 & 0,33 & 0,60 \\
1973 & $-0,38$ & $-0,94$ & $-0,11$ & $-0,24$ & $-0,05$ & $-0,07$ \\
1974 & 0,65 & 1,59 & 0,62 & 1,57 & 0,69 & 1,74 \\
1975 & 3,11 & 5,42 & 2,87 & 4,80 & 2,98 & 5,08 \\
1976 & 1,65 & 2,22 & 1,84 & 2,59 & 2,06 & 3,05 \\
1977 & 1,22 & 1,08 & 1,22 & 1,05 & 1,37 & 1,37 \\
1978 & 1,15 & 1,14 & 1,46 & 1,77 & 1,57 & 1,97 \\
1979 & 0,83 & 1,22 & 1,33 & 2,17 & 1,38 & 2,25 \\
1980 & 1,05 & 2,22 & 1,35 & 2,78 & 1,31 & 2,68 \\
1981 & 1,84 & 3,66 & 1,86 & 3,67 & 1,76 & 3,46 \\
1982 & 1,81 & 3,31 & 1,56 & 2,72 & 1,48 & 2,56 \\
\hline
\end{tabular}

Quelle: Statistisches Bundesamt (1994a, 1994b) sowie interne Unterlagen, Sachverständigenrat zur Begutachtung der gesamtwirtschaftlichen Entwicklung (1995); eigene Berechnungen.

Ein Vergleich der Primärüberschußlücken für Bund und Gesamthaushalt zeigt ähnliche Verläufe für die Zeit von 1969 bis 1982. Allen Gebietskörperschaften ist gemein, daß das Jahr 1969 aus Sicht der Schuldensituation äuBerst günstig war. Übereinstimmungen ergeben sich auch in einem mehr oder minder abrupten Anstieg der Indikatoren 1974/75 mit anschließend rückläufiger Tendenz. Während der Anstieg 1975 jedoch beim Bund ausgeprägter war als im Gesamthaushalt und die Primärüberschußlücken langsamer zurückgingen, ist die Verschlechterung zum Ende der 70er Jahre hin vor allem auf die Länderhaushalte zurückzuführen. Bei Bund und Ländern lassen sich insgesamt für die Zeit von 1969 bis 1982 deutlichere Parallelen feststellen als bei diesen in Bezug zu den Gemeinden. Neben höheren Lücken in 
1970/71 weichen die Indikatoren der Länder nur in ihrer größeren Variabilität ab Mitte der 70er Jahre (auf einen schnelleren Abbau der Lücken nach der Rezession 1975 folgt ein ebenfalls schnellerer Anstieg im Zuge der zweiten ölpreisbedingten Rezession) von denen des Bundes ab. Grundsätzlich beschreiben die Lücken für beide einen ähnlichen Verlauf. Die negative Entwicklung des Bundes wird also im Rahmen des Gesamthaushalts durch die Länder noch verstärkt. Im Gegensatz zu Bund und Ländern verzeichnen die Gemeinden ihre höchsten Indikatoren nicht 1974/75, sondern zu Beginn der 70er Jahre, während die Position nach 1975 für die Gemeinden deutlich besser ist als bei den zentralen Gebietskörperschaften. Die Gemeinden sind auch die wesentliche Ursache dafür, daß die Schuldensituation des Gesamthaushalts zu Beginn der 70er Jahre nicht so gut ausfällt wie die des Bundes.

Auch im Vergleich über den Zeitraum der 60er Jahre bis heute zeigen sich zwischen Bund, Ländern und Gemeinden deutliche Unterschiede. Während die Schuldensituation für den Bund in den 60er Jahren recht günstig aussah, überwiegt bei Ländern und Gemeinden in den $60 \mathrm{er}$ Jahren eine nicht tragfähige Politik. Die Situation des Gesamthaushalts fällt entsprechend schlechter aus als die des Bundes. Die Differenzen zwischen den 60er und 70er Jahren fallen gleichwohl für den Gesamthaushalt nach wie vor deutlich aus, da der Anstieg des Bundes, wie bereits gesagt, durch die Länderentwicklung noch deutlicher herausgestrichen wird. Im Laufe der 80er Jahre ergibt sich für alle Gebietskörperschaften eine Verbesserung der Tragfähigkeitsindikatoren, wobei diese für die Gemeinden am deutlichsten ausfällt. Im Gesamtbild der Primärüberschußlücken gehen die Gemeinden jedoch unter. Der Gesamthaushalt spiegelt vor allem die Entwicklung des Bundes und der Länder wider.

Für den Bund wurde bereits analysiert, in welche Richtung und in welchem Maße die beiden Seiten des Haushalts jeweils die Tragfähigkeit beeinflussen. Es zeigte sich hierbei, daß zu Beginn der 70er Jahre sowohl Steigerungen der Ausgaben als auch Verringerungen der Einnahmen zu einer Verschlechterung der Haushaltssituation beigetragen haben, insbesondere aber erstere. Die ausgabenbedingte Verschlechterung des Bundes wird im Gesamthaushalt durch ebenfalls steigende Ausgabenquoten bei den Gemeinden (allerdings in abnehmendem Maße) und insbesondere bei den Ländern noch verstärkt, was sich an den gegenüber den Primärüberschußlücken höheren 
Einnahmenlücken zeigt. Während die Einnahmenseite beim Bund zu einer Verschlechterung der Schuldensituation gegenüber dem Status quo geführt hat, läßt sich für den Gesamthaushalt keine eindeutige Aussage treffen. Darin spiegelt sich wider, daß die negative Entwicklung beim Bund durch einnahmenseitige Verbesserungen bei Ländern und Gemeinden größtenteils kompensiert wurde. Für Länder und Gemeinden liegen die mittel- und langfristigen Ausgabenlücken zu Beginn der 70er Jahre nicht nur unterhalb der Primärüberschußlücken, sie sind für 1969 bis 1973 auch fast durchweg negativ. Die Ursache für die unterschiedliche Entwicklung der Gebietskörperschaften liegt u.a. in dem sinkenden Anteil des Bundes am Steueraufkommen und einem steigenden Anteil der nachgeordneten Gebietskörperschaften. Dies würde auch erklären, warum sich beim Gesamthaushalt keine eindeutige einnahmenseitige Entwicklung im Vergleich zum Status quo feststellen läßt.

Ab Mitte der 70er Jahre tragen beim Bund zunächst sowohl Einnahmen- als auch Ausgabenseite zu einer - wenn auch geringen - Verbesserung der Schuldensituation bei, Ende der 70er Jahre ist es lediglich noch die einnahmenseitige Entwicklung. Anfang der 80 er Jahre sind beim Szenario 4 sowie bei den Szenarien 6 und 8 (die jeweils auf den Trends der Jahre 1976 bis 1982 basieren) ausgaben- bzw. einnahmenseitige Verbesserungen gegenüber dem Status quo festzustellen. Die im Vergleich zum Status quo festzustellenden tendenziellen einnahmenseitigen Verbesserungen beim Gesamthaushalt basieren vor allem auf der Entwicklung des Bundes, denn Länder und Gemeinden verzeichnen hier eher eine Verschlechterung. Die Entwicklung auf der Ausgabenseite trägt dagegen bei den Ländern eher zu einer Verbesserung der Schuldensituation bei: Nach Verschlechterungen von Mitte bis Ende der 70er Jahre ist dies Anfang der 80er Jahre auch für die Gemeinden der Fall. Der tendenziell negative Einfluß der Ausgabenentwicklung auf die Tragfähigkeit des Gesamthaushalts beruht daher im wesentlichen auf der Entwicklung des Bundes. Die beim Bund beschriebene Umorientierung, die sich, wie gezeigt wurde, durchweg auch in der Entwicklung der Tragfähigkeitsindikatoren niederschlägt, läßt sich bei Betrachtung der Szenario-Werte des Gesamthaushalts nicht feststellen. Lediglich bei den Einnahmenlücken läßt sich beim Vergleich der Szenarien 3 und 4 (die den Trend der Ausgabenquote für 1970 bis 1982 bzw. 1976 bis 1982 reflektieren) eine deutliche Verbesserung des Ausgabengebarens nach 1976 feststellen. Aber selbst im 
günstigsten Szenario trägt die Ausgabenseite noch zu einer Verschlechterung der Schuldensituation gegenüber dem Status quo bei. Auch bei einer Differenzierung ausgaben- und einnnahmenseitiger Komponenten an der Entwicklung der Tragfähigkeitsindikatoren zeigt sich mithin, daß der Gesamthaushalt insbesondere die Entwicklung des Bundes reflektiert.

Es wurde zusätzlich untersucht, ob die beschriebenen Ergebnisse relativiert werden müssen, wenn man die Finanzierungslücken in Relation setzt zum

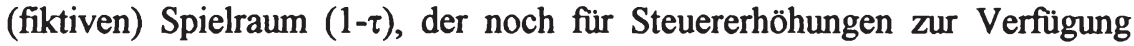
steht. Dabei wurde dieser Spielraum für den Bund jeweils mit seinem Anteil am Gesamtsteueraufkommen gewichtet. Dem liegt die Überlegung zugrunde, da $B$ die Konsolidierungschancen tendenziell um so geringer sind, je höher die bereits erreichte Steuerquote (Steuereinnahmen in v.H. des Bruttosozialprodukts) ist. Die bereits beschriebenen Ergebnisse werden im wesentlichen bestätigt. Gemessen an den so gewichteten Einnahmenlücken sind die Konsolidierungschancen für den Bund nach 1976 besser als für den Gesamthaushalt. Bei Betrachtung der gewichteten Ausgabenlücken fällt dagegen der Vergleich zuungunsten des Bundes aus. 
Tabelle 4: Einnahmen- und Ausgabenlücken des Bundes und des Gesamthaushalts im Vergleich

\begin{tabular}{|c|c|c|c|c|c|c|c|c|}
\hline \multirow{3}{*}{ Jahr } & \multicolumn{8}{|c|}{$\begin{array}{l}\text { Mittelfristige Einnahmenlücken } \\
\text { (in v.H. des Bruttosozialprodukts) }\end{array}$} \\
\hline & \multicolumn{2}{|c|}{ Szenario 1} & \multicolumn{2}{|c|}{ Szenario 2} & \multicolumn{2}{|c|}{ Szenario 3} & \multicolumn{2}{|c|}{ Szenario 4} \\
\hline & Bund & $\begin{array}{l}\text { Gesamt- } \\
\text { haushalt }\end{array}$ & Bund & $\begin{array}{l}\text { Gesamt- } \\
\text { haushalt }\end{array}$ & Bund & $\begin{array}{l}\text { Gesamt- } \\
\text { haushalt }\end{array}$ & Bund & $\begin{array}{l}\text { Gesamt- } \\
\text { haushalt }\end{array}$ \\
\hline 1969 & $-1,47$ & $-1,94$ & $-1,47$ & $-1,94$ & $-1,47$ & $-1,94$ & $-1,47$ & $-1,94$ \\
\hline 1970 & $-0,43$ & 0,72 & $-0,43$ & 0,72 & $-0,43$ & 0,72 & $-0,43$ & 0,72 \\
\hline 1971 & 0,35 & 2,03 & 0,35 & 2,03 & 0,35 & 2,03 & 0,35 & 2,03 \\
\hline 1972 & 0,73 & 1,78 & 0,73 & 1,78 & 0,73 & 1,78 & 0,73 & 1,78 \\
\hline 1973 & 0,79 & 1,69 & 0,79 & 1,69 & 0,79 & 1,69 & 0,79 & 1,69 \\
\hline 1974 & 1,42 & 2,20 & 1,42 & 2,20 & 1,42 & 2,20 & 1,42 & 2,20 \\
\hline 1975 & 2,01 & 3,18 & 2,01 & 3,18 & 2,01 & 3,18 & 2,01 & 3,18 \\
\hline 1976 & 1,59 & 2,44 & 1,59 & 2,44 & 1,59 & 2,44 & 1,59 & 2,44 \\
\hline 1977 & 1,31 & 1,71 & 1,31 & 1,71 & 1,31 & 1,71 & 1,31 & 1,71 \\
\hline 1978 & 1,37 & 2,14 & 1,37 & 2,14 & 1,37 & 2,14 & 1,37 & 2,14 \\
\hline 1979 & 1,46 & 2,77 & 1,44 & 2,79 & 1,49 & 2,85 & 1,40 & 2,72 \\
\hline 1980 & 1,67 & 3,00 & 1,62 & 3,03 & 1,77 & 3,22 & 1,56 & 2,93 \\
\hline 1981 & 1,99 & 3,76 & 1,91 & 3,82 & 2,15 & 4,15 & 1,80 & 3,67 \\
\hline 1982 & 1,69 & 3,18 & 1,58 & 3,26 & 1,97 & 3,84 & 1,45 & 3,14 \\
\hline
\end{tabular}




\begin{tabular}{|c|c|c|c|c|c|c|c|c|}
\hline \multirow{3}{*}{ Jahr } & \multicolumn{8}{|c|}{$\begin{array}{l}\text { Mittelfristige Ausgabenlücken } \\
\text { (in v.H. des Bruttosozialprodukts) }\end{array}$} \\
\hline & \multicolumn{2}{|c|}{ Szenario 5} & \multicolumn{2}{|c|}{ Szenario 6} & \multicolumn{2}{|c|}{ Szenario 7} & \multicolumn{2}{|c|}{ Szenario 8} \\
\hline & Bund & $\begin{array}{l}\text { Gesamt- } \\
\text { haushalt }\end{array}$ & Bund & $\begin{array}{l}\text { Gesamt- } \\
\text { haushalt }\end{array}$ & Bund & $\begin{array}{l}\text { Gesamt- } \\
\text { haushalt }\end{array}$ & Bund & $\begin{array}{l}\text { Gesamt- } \\
\text { haushalt }\end{array}$ \\
\hline 1969 & $-0,30$ & $-1,59$ & $-0,30$ & $-1,59$ & $-0,30$ & $-1,59$ & $-0,30$ & $-1,59$ \\
\hline 1970 & $-0,60$ & $-1,31$ & $-0,60$ & $-1,31$ & $-0,60$ & $-1,31$ & $-0,60$ & $-1,31$ \\
\hline 1971 & $-0,09$ & $-0,03$ & $-0,09$ & $-0,03$ & $-0,09$ & $-0,03$ & $-0,09$ & $-0,03$ \\
\hline 1972 & 0,58 & 0,33 & 0,58 & 0,33 & 0,58 & 0,33 & 0,58 & 0,33 \\
\hline 1973 & 0,35 & $-0,07$ & 0,35 & $-0,07$ & 0,35 & $-0,07$ & 0,35 & $-0,07$ \\
\hline 1974 & 0,78 & 1,68 & 0,78 & 1,68 & 0,78 & 1,68 & 0,78 & 1,68 \\
\hline 1975 & 2,49 & 3,86 & 2,49 & 3,86 & 2,49 & 3,86 & 2,49 & 3,86 \\
\hline 1976 & 1,44 & 1,73 & 1,44 & 1,73 & 1,44 & 1,73 & 1,44 & 1,73 \\
\hline 1977 & 1,11 & 1,17 & 1,11 & 1,17 & 1,11 & 1,17 & 1,11 & 1,17 \\
\hline 1978 & 1,36 & 1,82 & 1,36 & 1,82 & 1,36 & 1,82 & 1,36 & 1,82 \\
\hline 1979 & 1,27 & 1,97 & 1,20 & 1,91 & 1,30 & 1,95 & 1,22 & 1,97 \\
\hline 1980 & 1,31 & 2,73 & 1,15 & 2,62 & 1,35 & 2,64 & 1,17 & 2,70 \\
\hline 1981 & 1,74 & 3,17 & 1,48 & 2,97 & 1,81 & 3,01 & 1,50 & 3,10 \\
\hline 1982 & 1,80 & 2,74 & 1,42 & 2,46 & 1,87 & 2,42 & 1,41 & 2,55 \\
\hline
\end{tabular}


Fortsetzung Tabelle 4

\begin{tabular}{|c|c|c|c|c|c|c|c|c|}
\hline \multirow{3}{*}{ Jahr } & \multicolumn{8}{|c|}{$\begin{array}{l}\text { Langfristige Einnahmenlücken } \\
\text { (in v.H. des Bruttosozialprodukts) }\end{array}$} \\
\hline & \multicolumn{2}{|c|}{ Szenario 1} & \multicolumn{2}{|c|}{ Szenario 2} & \multicolumn{2}{|c|}{ Szenario 3} & \multicolumn{2}{|c|}{ Szenario 4} \\
\hline & Bund & $\begin{array}{l}\text { Gesamt- } \\
\text { haushalt }\end{array}$ & Bund & $\begin{array}{l}\text { Gesamt- } \\
\text { haushalt }\end{array}$ & Bund & $\begin{array}{l}\text { Gesamt- } \\
\text { haushalt }\end{array}$ & Bund & $\begin{array}{l}\text { Gesamt- } \\
\text { haushalt }\end{array}$ \\
\hline 1969 & $-0,65$ & 0,01 & $-0,65$ & 0,01 & $-0,65$ & 0,01 & $-0,65$ & 0,01 \\
\hline 1970 & 0,34 & 2,19 & 0,34 & 2,19 & 0,34 & 2,19 & 0,34 & 2,19 \\
\hline 1971 & 0,63 & 2,62 & 0,63 & 2,62 & 0,63 & 2,62 & 0,63 & 2,62 \\
\hline 1972 & 0,88 & 2,25 & 0,88 & 2,25 & 0,88 & 2,25 & 0,88 & 2,25 \\
\hline 1973 & 0,91 & 2,13 & 0,91 & 2,13 & 0,91 & 2,13 & 0,91 & 2,13 \\
\hline 1974 & 1,45 & 2,53 & 1,44 & 2,54 & 1,47 & 2,57 & 1,42 & 2,50 \\
\hline 1975 & 2,07 & 3,68 & 2,05 & 3,70 & 2,12 & 3,79 & 2,01 & 3,65 \\
\hline 1976 & 1,93 & 3,31 & 1,89 & 3,34 & 2,01 & 3,49 & 1,84 & 3,27 \\
\hline 1977 & 1,61 & 2,38 & 1,56 & 2,42 & 1,74 & 2,69 & 1,50 & 2,36 \\
\hline 1978 & 1,62 & 2,66 & 1,55 & 2,72 & 1,80 & 3,10 & 1,47 & 2,66 \\
\hline 1979 & 1,62 & 3,06 & 1,54 & 3,14 & 1,89 & 3,69 & 1,46 & 3,12 \\
\hline 1980 & 1,67 & 2,93 & 1,56 & 3,03 & 2,06 & 3,85 & 1,51 & 3,13 \\
\hline 1981 & 1,85 & 3,48 & 1,73 & 3,60 & 2,29 & 4,53 & 1,67 & 3,72 \\
\hline 1982 & 1,56 & 2,93 & 1,42 & 3,05 & 2,06 & 4,12 & 1,35 & 3,19 \\
\hline
\end{tabular}




\begin{tabular}{|c|c|c|c|c|c|c|c|c|}
\hline \multirow{3}{*}{ Jahr } & \multicolumn{8}{|c|}{$\begin{array}{l}\text { Langfristige Ausgabenlücken } \\
\text { (in v.H. des Bruttosozialprodukts) }\end{array}$} \\
\hline & \multicolumn{2}{|c|}{ Szenario 5} & \multicolumn{2}{|c|}{ Szenario 6} & \multicolumn{2}{|c|}{ Szenario 7} & \multicolumn{2}{|c|}{ Szenario 8} \\
\hline & Bund & $\begin{array}{l}\text { Gesamt- } \\
\text { haushalt }\end{array}$ & Bund & $\begin{array}{l}\text { Gesamt- } \\
\text { haushalt }\end{array}$ & Bund & $\begin{array}{l}\text { Gesamt- } \\
\text { haushalt }\end{array}$ & Bund & $\begin{array}{l}\text { Gesamt- } \\
\text { haushalt }\end{array}$ \\
\hline 1969 & 0,32 & $-1,26$ & 0,32 & $-1,26$ & 0,32 & $-1,26$ & 0,32 & $-1,26$ \\
\hline 1970 & $-0,24$ & $-1,26$ & $-0,24$ & $-1,26$ & $-0,24$ & $-1,26$ & $-0,24$ & $-1,26$ \\
\hline 1971 & $-0,01$ & $-0,34$ & $-0,01$ & $-0,34$ & $-0,01$ & $-0,34$ & $-0,01$ & $-0,34$ \\
\hline 1972 & 0,56 & 0,08 & 0,56 & 0,08 & 0,56 & 0,08 & 0,56 & 0,08 \\
\hline 1973 & 0,27 & $-0,23$ & 0,27 & $-0,23$ & 0,27 & $-0,23$ & 0,27 & $-0,23$ \\
\hline 1974 & 0,66 & 1,55 & 0,62 & 1,52 & 0,67 & 1,54 & 0,63 & 1,55 \\
\hline 1975 & 2,44 & 3,83 & 2,36 & 3,77 & 2,46 & 3,78 & 2,37 & 3,81 \\
\hline 1976 & 1,56 & 1,99 & 1,43 & 1,90 & 1,59 & 1,92 & 1,44 & 1,96 \\
\hline 1977 & 1,21 & 1,33 & 1,03 & 1,20 & 1,25 & 1,18 & 1,03 & 1,24 \\
\hline 1978 & 1,49 & 1,87 & 1,25 & 1,70 & 1,53 & 1,65 & 1,24 & 1,74 \\
\hline 1979 & 1,39 & 1,96 & 1,08 & 1,74 & 1,42 & 1,63 & 1,04 & 1,74 \\
\hline 1980 & 1,37 & 2,69 & 0,99 & 2,41 & 1,37 & 2,15 & 0,88 & 2,29 \\
\hline 1981 & 1,76 & 3,07 & 1,33 & 2,76 & 1,76 & 2,46 & 1,20 & 2,62 \\
\hline 1982 & 1,83 & 2,73 & 1,34 & 2,38 & 1,83 & 2,03 & 1,19 & 2,22 \\
\hline
\end{tabular}

Quelle: Statistisches Bundesamt (1994a, 1994b) sowie interne Unterlagen, Sachverständigenrat zur Begutachtung der gesamtwirtschaftlichen Entwicklung (1995); eigene Berechnungen. 


\subsection{EinfluB der Finanzpolitik auf Wachstum und Zinsen und ihre Auswirkungen auf die Tragfähigkeit der Verschuldung}

In den vorangegangenen Abschnitten wurde die Finanzpolitik der sozial-liberalen Koalition eingehend auf ihre Tragfähigkeit hin analysiert. Grundsätzlich können die Tragfähigkeitsindikatoren als ex post- oder als ex ante$\mathrm{Maße}$, d.h. zur Beurteilung einer vergangenen bzw. einer prognostizierten Schuldenpolitik herangezogen werden. ${ }^{180}$ Hier wurde gewissermaßen eine Mischung aus beidem vorgenommen. Zum einen wurden die in der Zeit von 1969 bis 1982 realisierten Haushalts- und Verschuldungsdaten zur Berechnung herangezogen. Gleichzeitig wurde aber in Form von Szenarien - gewissermaßen aus der Vergangenheit heraus - in die Zukunft prognostiziert. Die „Prognose“ bezog sich allerdings allein auf die Entwicklung der Einnahmen und Ausgaben bzw. Einnahmen- und Ausgabenquoten. Für das Wachstum des Bruttosozialprodukts und die Zinsentwicklung wurde zunächst angenommen, daß sie durch die Szenarien nicht beeinflußt werden, d.h. es wurden trotz Unterschieden in der auf den Szenarien basierenden und der nach 1982 tatsächlich realisierten Haushaltspolitik die tatsächlichen Werte angesetzt. Auf eine Trendbildung wurde verzichtet, da die Interdependenzen zwischen Haushalts- und Wirtschaftsentwicklung zu vielfältig sind. Eine voneinander unabhängige „Prognose“ für Einnahmen und Ausgaben sowie Wachstum und (rechnerischen) Zins hätte die Ergebnisse eher verfälscht und die Aussagekraft eingeschränkt. Dennoch müssen die Resultate im folgenden relativiert werden.

Dadurch, daß rechnerischer Zins und Wachstumsrate in Form der tatsächlich realisierten Werte exogen vorgegeben wurden, blieb somit unberücksichtigt, wie sich die Entwicklung der Haushalte bzw. der Schulden auf diese Größen auswirkt. Durch das öffentliche Defizit wird das Nettokapitalangebot für die Privatwirtschaft verringert. Dies ließe einen Anstieg der Zinsen vermuten. Empirische Betrachtungen liefern diesbezüglich jedoch kein übereinstimmendes Ergebnis. Dem Anschein nach ist ein solcher Zusammenhang, so er denn existiert, eher bei den langfristigen Zinsen zu finden, während die kurzfristigen Zinsen davon weniger betroffen zu sein scheinen. ${ }^{181}$ In einer offe-

180 Eine reine ex post-Analyse kann jedoch einfacher durch eine Betrachtung der Schuldenquote, der Zinsentwicklung etc. durchgeführt werden.

181

Vgl. Boss/Lorz (1995), S. 18 f. 
nen Volkswirtschaft mit einem leistungsfähigen Bankensystem wie der Bundesrepublik Deutschland sollte zudem das Geldangebot relativ elastisch reagieren. ${ }^{182}$ Ein Anstieg der Zinsen kann nicht ausgeschlossen werden, ist jedoch nicht zwingend. So kann Evans ${ }^{183}$ im Rahmen einer Untersuchung von sechs OECD-Staaten, darunter die Bundesrepublik Deutschland, keinen zinstreibenden Effekt der Staatsverschuldung feststellen.

Die Wirkung der Verschuldung auf die Wachstumsrate ist noch weniger offensichtlich. In den Anfängen des Stabilitäts- und Wachstumsgesetzes war man zunächst optimistisch davon ausgegangen, daß sich ein Deficit Spending mehr oder weniger über ein höheres Wachstum selber finanzieren würde. Wie sich die öffentliche Verschuldung auf das Wachstum auswirkt, wird zum einen davon abhängen, ob die aufgenommenen Kreditmittel investiv oder konsumtiv verwendet werden. Eine Erhöhung des Staatsverbrauchs wird sich eher negativ auf das Wachstum des Bruttosozialprodukts auswirken. Dies wird durch eine neuere Untersuchung von Barro und Sala-I-Martin tendenziell bestätigt. ${ }^{184}$ Im Falle öffentlicher Investitionen ist eher ein positiver Effekt zu vermuten. Theoretische und empirische Untersuchungen insbesondere zu den ökonomischen Wirkungen öffentlicher Infrastrukturinvestitionen - kommen jedoch zu recht unterschiedlichen Ergebnissen. ${ }^{185} \mathrm{Die}$ Höhe des Schuldenstandes und sein Wachstumstempo kann sich ebenfalls auf das Wachstum auswirken. Entsteht der Eindruck, der Staat habe seinen Haushalt nicht mehr im Griff, dann werden negative Erwartungen zu einem Sinken der Wachstumsrate führen. Dies war zu einem gewissen Grade gegen Ende der sozial-liberalen Koalition der Fall.

Zins und Wachstum wirken wiederum zurück auf den Haushalt, zum einen in Form der Zinsdynamik, zum anderen über die eingebauten Stabilisatoren, wobei von einem Mehr an Wachstum auch ein positiver Effekt auf den

182 Vgl. Rürup (1982), S. $630 \mathrm{f}$.

183

184

Vgl. Evans (1987).

Vgl. Barro/Sala-I-Martin (1995), S. 414-461, insbesondere S. 434.

$185 \mathrm{Vgl}$. etwa Aschauer (1989), Conrad/Seitz (1994) sowie Ludvigson (1996), die zu einem recht positiven Ergebnis bezogen auf Produktivität und Wachstum gelangen, im Gegensatz zu bspw. Holtz-Eakin/Schwartz (1995) und Crihfield/Panggabean (1995), deren Ergebnisse pessimistisch ausfallen. Allerdings betrachten lediglich Conrad/Seitz die Bundesrepublik. 
Tabelle 5: Langfristige Primärüberschußlücken bei alternativen Zins-Wachstums-Differentialen für die Jahre 1983 bis 1991

\begin{tabular}{|c|c|c|c|c|c|c|c|c|c|c|}
\hline \multirow{3}{*}{ Jahr } & \multicolumn{10}{|c|}{ in v.H. des Bruttosozialprodukts } \\
\hline & \multicolumn{10}{|c|}{$\begin{array}{l}\text { Zins-Wachstums-Differential } \\
\text { (in v.H.) }\end{array}$} \\
\hline & $\begin{array}{l}\text { tatsächl } \\
\text { Werte }\end{array}$ & $-2,0$ & $-1,0$ & 0,0 & 0,5 & 1,0 & 2,0 & 3,0 & 4,0 & 5,0 \\
\hline 1969 & $-0,82$ & $-0,82$ & $-0,82$ & $-0,82$ & $-0,82$ & $-0,82$ & $-0,82$ & $-0,82$ & $-0,82$ & $-0,82$ \\
\hline 1970 & $-0,62$ & $-0,62$ & $-0,62$ & $-0,62$ & $-0,62$ & $-0,62$ & $-0,62$ & $-0,62$ & $-0,62$ & $-0,62$ \\
\hline 1971 & & -0 & $-0,29$ & $-0,29$ & $-0,29$ & $-0,29$ & $-0,29$ & $-0,29$ & $-0,29$ & -0 \\
\hline 1972 & 3 & 0,33 & 0,33 & 0,33 & 0,33 & 0,33 & 0,33 & 0,33 & 0,33 & 0,33 \\
\hline 1973 & $-0,05$ & $-0,05$ & $-0,05$ & $-0,05$ & $-0,05$ & $-0,05$ & $-0,05$ & $-0,05$ & $-0,05$ & $-0,05$ \\
\hline 1974 & 0,69 & 0.66 & 0,67 & 0,67 & 0,68 & 0,68 & 0,68 & 0,69 & 0,70 & 0,70 \\
\hline 1975 & 8 & 2,92 & 2,93 & 2,95 & 2,95 & 2,96 & 2,97 & 2,99 & 3,00 & 3,01 \\
\hline 1976 & & 1,91 & 1,94 & 1,97 & 1,98 & 2,00 & 2,03 & 2,06 & 2,09 & 2,12 \\
\hline 1977 & 1,37 & 1,16 & 1,21 & 1,25 & 1,27 & 1,30 & 1,34 & 1,38 & 1,42 & 1,46 \\
\hline 1978 & 1,57 & 1 , & 1,32 & 1,39 & 1,42 & 1,45 & 1,51 & 1,57 & 1,62 & 1,68 \\
\hline 1979 & 1,38 & 0,99 & 1,07 & 1,15 & 1,19 & 1,23 & 1,32 & 1,40 & 1,48 & 1,55 \\
\hline 1980 & 1,31 & 0,85 & 0,95 & 1,05 & 1,11 & 1,16 & 1,26 & 1,36 & 1,46 & 1,56 \\
\hline 1981 & 1,69 & 1,17 & 1,30 & 1,43 & 1,49 & 1,56 & 1,69 & 1,82 & 1,95 & 2,08 \\
\hline 1982 & 1,19 & 0,74 & 0,90 & 1,06 & 1,14 & 1,23 & 1,39 & 1,55 & 1,72 & 1,89 \\
\hline
\end{tabular}

Anmerkung: Aus Gründen der Vergleichbarkeit ist im Gegensatz zu Tabelle 3 für die Primärüberschußlücken der Jahre 1981 und 1982 auf Basis der tatsächlichen Werte ebenfalls ein zehnjăhriger Zeithorizont zugrundegelegt.

Quelle: Statistisches Bundesamt (1994a, 1994b) sowie interne Unterlagen, Sachverstăndigenrat zur Begutachtung der gesamtwirtschaflichen Entwicklung (1995); eigene Berechnungen.

Haushalt zu erwarten ist. Prinzipiell kann die Frage, wie sich die durch die Szenario-Bildung vorgenommenen Änderungen des Haushalts auf die Zinsdynamik auswirken, nur in einem geschlossenen dynamischen Modell beantwortet werden, das die Interdependenzen zwischen Finanzpolitik, Sozialprodukts- und Zinsentwicklung erfaßt, wobei innerhalb eines umfassenderen Modells zusätzlich Informationen über die Haushaltsstruktur notwendig würden, die bei der Tragfähigkeitsbetrachtung selbst gar nicht berücksichtigt werden. Eine empirische Analyse auf der Basis eines solchen Modells liegt jedoch außerhalb des Rahmens dieser Studie. Tendenziell kann angesichts der Größenordnung der für die sozial-liberale Koalition berechneten Tragfähigkeitsindikatoren und unter Berücksichtigung der Tatsache, daß der kon- 
sumtive Anteil des Haushalts zu Lasten des investiven Anteils an Gewicht gewonnen hat, eher von einem höheren als dem bei den vorangegangenen Berechnungen unterstellten Zins-Wachstums-Differential ausgegangen werden. In Tabelle 5 ist die langfristige Primärüberschußlücke des Jahres 1982 für alternative Zins-Wachstums-Differentiale abgebildet. Wäre z.B. nach 1982 ein Zins-Wachstums-Differential von durchschnittlich $2 \%$ realisiert worden, dann wären im Jahre 1982 Anpassungsmaßnahmen in Höhe von $1,39 \%$ des Bruttosozialprodukts (über die nächsten 10 Jahre) notwendig gewesen, um die Schuldenquote zu stabilisieren, d.h. 0,2 Prozentpunkte mehr als bei den tatsächlich realisierten Werten des Zins-Wachstums-Differentials. 


\subsection{Geldpolitische Aspekte der Verschuldung}

Bei den bisherigen Überlegungen wurden Preisniveauveränderungen nicht explizit thematisiert. Im folgenden soll daher untersucht werden, wie sich die finanzielle Situation der öffentlichen Haushalte, insbesondere des Bundes, bei Berücksichtigung der Inflation ändert. In der Literatur galt der Rolle der Finanzpolitik in bezug auf die Geldpolitik stets besonderes Interesse. Die Diskussion um die europäische Wirtschafts- und Währungsunion hat diesem Problembereich in jüngster Zeit erneut Auftrieb gegeben. ${ }^{186}$

$\mathrm{Da}$ die 70er Jahre eine Periode hoher Preissteigerungsraten darstellen wenngleich im internationalen Vergleich am unteren Ende der Skala -, muß die Inflation in die Betrachtung mit einbezogen werden. Die durchschnittliche Preissteigerungsrate der Verbraucherpreise bzw. des Preisindex der Lebenshaltung lag bei $4,8 \%$, wobei sich die Jahreswerte innerhalb einer Bandbreite von 2 bis $7 \%$ bewegten. Demgegenüber waren die Preise in der Zeit von Ende 1960 bis 1969 im Schnitt ,lediglich“ um 3,1\% und von Ende 1983 bis 1994 um 2,4\% gestiegen. Das Schaubild 23 gibt diese Entwicklung im einzelnen nochmals wieder.

Sieht man von den Wirkungen der Inflation auf das realwirtschaftliche Geschehen ab, dann kann die Geldentwertung prinzipiell von drei Seiten Einfluß auf das Defizit bzw. die Verschuldung ausüben: über eine Änderung der realen Verzinsung der Staatsschuld, über eine Verteuerung der öffentlichen Güter bzw. des öffentlichen Konsums und über die sog. Seigniorage. Der über den Zins wirksame Effekt der Inflation auf die Schuldenquote hängt in hohem $\mathrm{Maße}$ von der Antizipation der Geldentwertung seitens der Staatsgläubiger ab. Wie oben gezeigt wurde, wird die Entwicklung der Schuldenquote wesentlich vom Zins-Wachstums-Differential beeinflußt. Erst einmal schlagen sich Preisänderungen voll in der nominalen Wachstumsrate nieder. Wie sich aber letztlich das Verhältnis von Zins und Wachstumsrate entwickelt, hängt davon ab, wie der Nominalzins auf die Preisentwicklung reagiert. Unterliegen die Gläubiger der Staatsschuld einer Geldillusion oder werden sie von der Inflation überrascht, dann bleibt der Nominalzins (unter

$186 \mathrm{Zu}$ den folgenden Ausführungen vgl. etwa Pfadt/Schröder (1994) oder Schlesinger/ Weber/Ziebarth, S. 48-67. 
sonst gleichen Bedingungen) zunächst konstant, und der Realzins sinkt. Mithin sinkt auch das Zins-Wachstums-Differential, und der Staat wird praktisch entschuldet.

Geht man davon aus, daß die Gläubiger im allgemeinen keiner Geldillusion unterliegen und entsprechend der von ihnen erwarteten Inflationsrate nur gegen eine höhere nominale Verzinsung bereit sind, dem Staat Kredite zur Verfügung zu stellen, dann wird die Inflation - zumindest in dem Maße, in dem eine Beeinflussung des Zinses durch die Gläubiger möglich ist - wieder auf den Staat zurückgewälzt. Bei vollständiger Antizipation der Preissteigerungen ändert sich gemäß dem Fisherschen Zinstheorem ${ }^{187}$ das Zins-Wachstums-Differential nicht, da der höheren Wachstumsrate ein gleichermaßen höherer Zins gegenübersteht. Bekanntlich hinkt jedoch die rechnerische Verzinsung der Staatsschuld dem allgemeinen Zinsniveau hinterher. Die durch längere Zinsbindungsfristen und Laufzeiten verzögerte Anpassung bewirkt somit eine temporäre Verringerung des Zins-Wachstums-Differentials und eine Senkung des realen Werts der öffentlichen Schuld, der praktisch von den Altgläubigern der Staatsschuld getragen wird. Da die Laufzeit öffentlicher Verschuldung in den 70er Jahren im Schnitt höher war als heute ${ }^{188}$, war eine zumindest partielle Entschuldung des Staates eher gegeben als heute. Vorübergehend erwuchs dem Fiskus aus der Inflation somit ein Vorteil. Eine dauerhafte Entlastung wäre jedoch nur über eine Akzeleration der Inflation möglich gewesen.

Wie stark der Staat letztlich von der Geldentwertung profitierte, läßt sich empirisch kaum quantifizieren. Hierzu müßte man die antizipierte Inflationsrate kennen, denn nur so läßt sich bestimmen, wie hoch die reale Verzinsung der Staatsschuld tatsächlich ausfiel. Durch die Variation der Inflationsrate über die Zeit ist eine Bestimmung des Realzinses zusätzlich erschwert. Eine systematische Nutzung des Inflationsmechanismus durch den Staat in der beschriebenen Art kann kaum angenommen werden; insbesondere nicht, wenn man bedenkt, daß die öffentliche Hand ebenfalls als Nachfrager auftritt und somit selbst negativ von den Preissteigerungen betroffen ist (vgl.

187 Dieses Zinstheorem kann jedoch nur als eine Annäherung betrachtet werden, da es gemessen an der Realität einige Probleme aufwirft, vgl. hierzu Gebauer (1982).

188 In der zweiten Hälfte der 70er Jahre wiesen durchschnittlich 39,5\% eine Laufzeit von 10 und mehr Jahren auf, vgl. Schlesinger/Weber/Ziebarth (1993), S. 181. 


\section{Preisentwicklung der Inlandsnachfrage}

Veränderung der Preisindices in v.H.

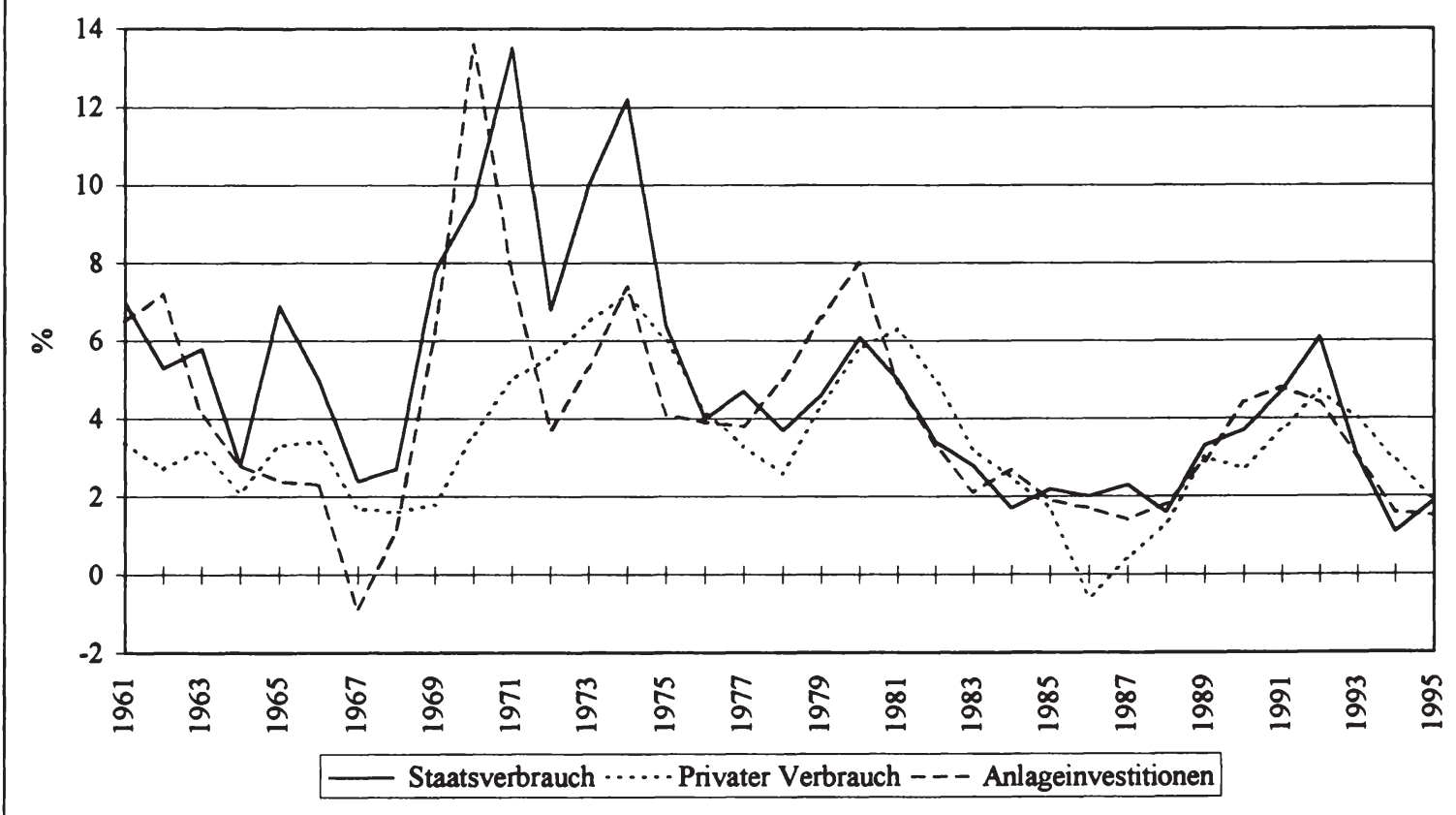

Anmerkung: Paasche-Preisindex mit wechselnder Gewichtung (Warenkorb des jeweiligen Berichtszeitraums). Ab 1991 vorlăfige Ergebnisse. Quelle: Sachverstăndigenrat zur Begutachtung der gesamtwirtschaftlichen Entwicklung $(1995,1996)$; eigene Berechnungen. 
Schaubild 23, wobei allerdings zu beachten ist, daß der Preisindex des Staatsverbrauchs auch die Änderungen in den Personalkosten des öffentlichen Sektors mit erfaßt). Das schließt nicht aus, daß nicht selten die Inflation durch das öffentliche Ausgabengebaren angeheizt wird. Für die 70er Jahre ist hier vor allem die prozyklische Wirkung des öffentlichen Haushalts auf den Bausektor zu nennen. ${ }^{189}$ Ein möglicher Vorteil aus der Inflation via Zinsmechanismus dürfte durch die negative Ausgabenentwicklung mehr als konterkariert werden.

Für die politischen Entscheidungsträger interessanter sind allemal die Gewinne, die aus der Schaffung von Geld entstehen, die sog. Seigniorage. $\mathrm{Zu}$ einem vernachlässigbar geringen Teil entstehen diese Einkünfte in der Bundesrepublik direkt beim Staat, und zwar über das sog. Münzregal. Der weitaus größere Teil entstammt jedoch dem Geldschöpfungsprozeß bei der Bundesbank. Seigniorage entsteht in erster Linie dadurch, da $\beta$ Bundesbank bzw. öffentliche Hand Banknoten bzw. Münzen ausgeben, deren Herstellungskosten unter dem Nennwert liegen. Zum einen fließen diese Mittel in den privaten Sektor, im Tausch gegen Güter und Dienstleistungen. Zum anderen sind die Geschäftsbanken verpflichtet, einen Teil ihrer Einlagen als Mindestreserve bei der Zentralbank zu hinterlegen. Da die Produktionskosten vernachlässigbar gering sind, wird die Seigniorage somit theoretisch durch die Veränderung der Geldbasis (Summe aus Bargeldumlauf und Einlagen der Geschäftsbanken bei der Zentralbank) bestimmt, die durch einen Preisindex deflationiert wird. Bei positiver Inflationsrate ist zwischen einer Seigniorage im engen Sinne und einer sog. Inflationsteuer zu unterscheiden.

Die Seigniorage im engeren Sinne, der passive Teil der Seigniorage, entspricht der Veränderung der Realkasse. Sie trägt der Tatsache Rechnung, $\mathrm{da} B$ in einer wachsenden Wirtschaft die geplanten Realkassenbestände im Zeitablauf steigen und kann daher als Wachstumskomponente interpretiert werden. Im Falle der Inflation kann das Niveau der Realkasse jedoch nur aufrecht erhalten werden, wenn die Nominalkassenbestände entsprechend dem Preisanstieg ausgedehnt werden. Dadurch entstehen dem Geldproduzenten Einkünfte in Höhe des Produktes aus Realgeldmenge und Inflationsrate. Dem Wesen nach entspricht dies einer Besteuerung der Geldbesitzer,

Vgl. Scherf (1986), S. 73-84. 
wobei der Realkassenbestand als Steuerbasis und die Inflationsrate als Steuersatz interpretiert werden können. Diese Einkünfte entstehen dem Geldanbieter auch dann, wenn die Inflationsrate von den Wirtschaftsakteuren vollständig antizipiert wird. Entscheidend ist, daß der Staat (direkt oder via Bundesbank) als Produzent und Anbieter von Geld eine Monopolstellung innehat. $^{190}$

Der budgetäre Inflationsansatz geht davon aus, daß die Inflationsrate zwar kurz- und mittelfristig die Bedingungen am Gütermarkt widerspiegelt, daß Inflation jedoch langfristig durch den Finanzierungsbedarf des Staates verursacht wird. ${ }^{191}$ Notenbank und Staat werden dabei als konsolidierte Einheit aufgefaßt, so daß die Seigniorage eine alternative Finanzierungsmöglichkeit des Defizits neben der Verschuldung darstellt. Da in der Bundesrepublik jedoch ein direkter Zugriff der öffentlichen Hand auf die Notenpresse ausgeschlossen werden kann, bleibt fragwürdig, inwieweit die öffentlichen Haushalte zu einer Inflationierung beigetragen hat. ${ }^{192}$ Der Sachverständigenrat gelangt in seinem Jahresgutachten 1980/81 zu dem Ergebnis, daß „die Staatsschuld von heute nicht inflationär finanziert worden ist". ${ }^{193}$ Neuman hat zudem gezeigt, daß der Anteil der Seigniorage, der dem Staat tatsächlich zufällt, entscheidend von rechtlichen, institutionellen und operationalen Details bei der Schaffung der monetären Basis abhängt. Da für die Bundesbank eine hinreichende Unabhängigkeit unterstellt werden kann, hat sie die Möglichkeit, den Umfang der Seigniorage, der dem Bund in Form des Bundesbankgewinns zufließt, zu beeinflussen. Im Gegensatz zur konservativ-liberalen Koalition hat die sozial-liberale Koalition nur in sehr geringem Umfang vom Geldschaffungsprozeß profitiert. Bis zum Jahre 1981 wurde praktisch gar kein Bundesbankgewinn abgeführt (vgl. Tabelle 6). Haupteinnahmequelle

190 Vgl. etwa Cornelius (1990), Institut „Finanzen und Steuern“ e.V. (1993) oder Neldner (1984).

191 In diesem Zusammenhang sind auch Modelle entwickelt worden, die eine für den Staat einnahmenmaximierende bzw. optimale Inflationsrate bestimmen, vgl. beispielsweise Tobin (1984) oder Cornelius (1990) und die dort angegebene Literatur.

192 Den Fall einer völlig von der Fiskalpolitik abhängigen Geldpolitik beschreiben beispielsweise Sargent/Wallace (1986). Zu Tragfähigkeitsuntersuchungen anhand konsolidierter Budgets vgl. auch Buti (1990), McCallum (1984) oder Spaventa (1987).

Sachverständigenrat zur Begutachtung der gesamtwirtschaftlichen Entwicklung (1980), Ziffer 295, so auch Caesar (1988), S. 47. 
der Bundesbank sind die Zinserträge. Diese waren in den 70er Jahren deutlich niedriger als in den $80 \mathrm{er}$ Jahren. Neben den Verwaltungskosten (sie stellen den größten Aufwandsposten dar) schlugen bei den Aufwendungen insbesondere die Wertberichtigungen auf Devisenreserven zu Buche, die aufgrund des sinkenden Dollars vorgenommen werden mußten. Insgesamt wurden daher zum Teil nicht unerhebliche Verluste erzielt. Mit Wegfall der Wertberichtigungen ${ }^{194}$ und steigendem Zinsniveau nahmen die Bundesgewinne schlagartig zu. ${ }^{195}$

Tabelle 6: Gewinnabführung der Bundesbank an den Bund

\begin{tabular}{|c|c|c|c|c|c|}
\hline Jahr & $\begin{array}{c}\text { Gewinn- } \\
\text { abführung } \\
\text { in Mrd. DM }\end{array}$ & Jahr & $\begin{array}{c}\text { Gewinn- } \\
\text { abführung } \\
\text { in Mrd. DM }\end{array}$ & Jahr & $\begin{array}{c}\text { Gewinn- } \\
\text { abführung } \\
\text { in Mrd. DM }\end{array}$ \\
\hline 1969 & 0,3 & 1978 & - & 1987 & 7,3 \\
1970 & - & 1979 & - & 1988 & 0,2 \\
1971 & 0,5 & 1980 & - & 1989 & 10,0 \\
1972 & - & 1981 & 2,3 & 1990 & 10,0 \\
1973 & - & 1982 & 10,5 & 1991 & 8,3 \\
1974 & - & 1983 & 11,0 & 1992 & 14,5 \\
1975 & - & 1984 & 11,4 & 1993 & 13,1 \\
1976 & 0,4 & 1985 & 12,9 & 1994 & 18,3 \\
1977 & - & 1986 & 12,7 & 1995 & 10,2 \\
\hline
\end{tabular}

Anmerkung: Die Angaben beziehen sich auf das Jahr, in dem der Bundesbankgewinn jeweils haushaltswirksam wurde.

Quelle: Deutsche Bundesbank, Geschăftsbericht, verschiedene Jahrgănge.

194 Lediglich 1987 sind wegen des niedrigen Dollarkurses nochmals Abschreibungen in 195 nennenswertem Umfang vorgenommen worden.

Vgl. Issing (1992), S. 204 f. 
Monika Hanswillemenke and Bernd Rahmann - 978-3-631-75268-5

Downloaded from PubFactory at 01/11/2019 06:29:54AM

via free access 


\section{Schlußfolgerungen}

Bei Regierungswechseln ist in aller Regel der Vorwurf zu hören, daß das Erbe der Politik der Vorgängerin den Handlungsspielraum der neuen Administration belastet. Handlungsspielraum kann dabei zunächst weit verstanden werden: zum einen bezüglich der proklamierten Ziele, zum anderen im Hinblick auf die einsetzbaren Einnahme- und Ausgabeninstrumente und schließlich unter Beachtung der fiskalischen Budgetrestriktionen. Kombinationen dieser Aspekte sind durchaus denkbar.

Da wäre zunächst die Möglichkeit zu nennen, die konsensfähigen nationalen Ziele - etwa die des Stabilitäts- und Wachstumsgesetzes - deklaratorisch beizubehalten, aber den instrumentellen Weg zur Zielerreichung zu wechseln. Diese Konstellation ist typisch für den Wechsel von der nachfrageorientierten zur angebotsorientierten Wirtschaftspolitik. Wird dabei aber wie z.B. im Falle der unter Präsident Ronald Reagan praktizierten Politik in den USA $^{196}$ - bei angebotswirksamen Steuersenkungen ein unvermindert hohes oder sogar noch steigendes öffentliches Budget kreditfinanziert und durch entsprechende Wechselkurspolitik die Auslandsverschuldung erhöht, so handelt es sich tatsächlich um eine zumindest partiell veränderte Zielsetzung, während aus der gesamtwirtschaftlichen Perspektive faktisch eine unveränderte Instrumentwirkung des Deficit Spending stattfindet. Es ist nicht auszuschließen, daß die gesamtwirtschaftlichen Effekte in Wirklichkeit von einer unveränderten Budgetpolitik miterzeugt werden. Lediglich die Budgetseiten werden gewechselt. Das kann oder soll auch verteilungspolitische Konsequenzen haben, was aber auch für die traditionelle Nachfragepolitik nicht ausgeschlossen ist (z.B. Senkung der Steuerlast bei konstanten Ausgaben mit dem beabsichtigten Makroeffekt steigender privater Kaufkraft). Mit Verweis auf die Langfristigkeit der zu erwartenden Wirkungen immunisiert sich eine solche Politik gegen Kritik hinsichtlich der kurzfristig ausbleibenden Effekte mit dem Hinweis, daß „die Politik der Verbesserung der Angebotsbedingungen langfristig angelegt sein müsse und daß daher die Zeit für sichtbare beschäftigungspolitische Erfolge noch zu kurz..." sei. ${ }^{197}$ Gleichermaßen immunisierend ist natürlich auch die Auffassung, daß kurzfristig an-

196 Siehe Friedman (1988), insbesondere Kap. V, VII und IX.

197 Buttler/Kühl/Rahmann (1985), im Vorwort der Herausgeber. 
gelegte antizyklische Maßnahmen nur deshalb nicht zum Erfolg geführt haben, weil sie nicht lange genug durchgehalten worden sind.

Die Wende in der Bundesrepublik Deutschland mit dem Regierungswechsel im Oktober 1982 kann als eine Spielart dieser Konstellation verstanden werden. Natürlich werden die im $\S 1$ StabWG vorgesehenen Ziele nicht ausdrücklich ausgetauscht. Das Gesetz bleibt offiziell ja auch in Kraft. Es findet aber eine andere Gewichtung statt, indem der Stabilität des Preisniveaus ein eindeutig höherer Rang eingeräumt wird als den übrigen Zielen. Die bekannte Maxime des abgelösten Bundeskanzlers Helmut Schmidt: „Fünf Prozent Inflation sind mir lieber als fünf Prozent Arbeitslosigkeit" ist jedenfalls nicht mehr handlungsleitend. Es ist allerdings anzumerken, daß auch bereits die sozial-liberale Koalition gegen Ende der 70er Jahre davon abrückte, die Phillipskurve als „Menu for Policy Choice“ anzusehen. Der nachfolgenden konservativ-liberalen Koalition ist es freilich strategisch gelungen, die hohe Arbeitslosigkeit nicht eigenen politischen Mißerfolgen zuzurechnen, sondern mit strukturellen und politisch nur langfristig zu bewältigenden Problemen zu charakterisieren. Konsequenter als die Vorgängerregierung setzt sie dabei auf eine erfolgreiche Rückführung der Staatsquote. Mit diesem Übergang zugunsten eines stärker betonten Allokationsziels gelingt ihr in der Tat die Wende im Sinne eines Ziel- und Instrumentwechsels - sogar zu Lasten einer unvermindert hohen (sogar noch steigenden) und sich verfestigenden Massenarbeitslosigkeit.

Es bleibt nun zu fragen, ob diese Wende durch die Schuldenpolitik der sozial-liberalen Regierung entscheidend behindert wurde; ob die Mißerfolge bei der Bekämpfung der andauernden Massenarbeitslosigkeit ursächlich verbunden sind mit dem Verschuldungsgrad der öffentlichen Hand; ob also fiskalische Budgetrestriktionen daran schuld sind, daß die von der konservativliberalen Regierungskoalition verfolgte Politik nicht zu einem höheren Beschäftigungsstand geführt hat. Entscheidend dafür ist die Perspektive, ob Ausgabenlasten infolge der früheren Verschuldung beschäftigungswirksame Finanzpolitik verhindert haben. In diesem Zusammenhang ist von der konservativ-liberalen Koalition in der Tat die These von der Erblast aufgeworfen worden. ${ }^{198}$

198 So beispielsweise in der Regierungserklärung von Helmut Kohl am 13. Oktober 1982, vgl. Kohl (1982), S. 858. 
Im vorangegangenen Kapitel wurde gezeigt, daß der Konsolidierungsbedarf im Sinne des Tragfähigkeitskriteriums seit Mitte der 70er Jahre insgesamt gestiegen war. Die Notwendigkeit einer Konsolidierung Anfang der 80er Jahre steht damit außer Frage. Der gewachsene Konsolidierungsbedarf stellte für sich genommen jedoch noch keine Erblast dar. Entscheidend ist, ob die Konsolidierungschancen sich ebenfalls im Laufe der 70er Jahre verschlechtert hatten und so möglicherweise eine tragfähige Finanzpolitik auf Dauer unmöglich machten. Außerdem ist zu prüfen, inwieweit die wachsende Zinslast zu einer Einschränkung des Ausgabenspielraums führte.

Grundsätzlich ist eine Haushaltsanpassung um so schwieriger, je höher - unter sonst gleichen Bedingungen - die erreichte Schuldenquote bzw. der Schuldenstand ist, da die Zinsdynamik stärker greift. Darüber hinaus ist, wie gezeigt wurde, das Zins-Wachstums-Differential im Zeitablauf tendenziell gestiegen, so daß die Eigendynamik der Verschuldung auch von dieser Seite her verstärkt wurde. Insgesamt sind also die Konsolidierungschancen eher gefallen als gestiegen. Nun wurde bei der bisherigen Untersuchung davon ausgegangen, daß die Schuldenquote jeweils auf dem bereits erreichten Niveau stabilisiert wird. Hätte man die Schuldenquote auf dem Niveau zu Beginn der 70er Jahre halten bzw. sie auf diesen Stand zurückführen wollen, dann hätte es weitaus größerer Anstrengungen bedurft. Exemplarisch sind die entsprechenden Primärüberschußlücken für eine konstante Schuldenquote von $7,1 \%$ des Bruttosozialprodukts berechnet worden. Dies entspricht der Schuldenquote des Bundes von 1970 und - nach einer zwischenzeitlichen Reduzierung und einem Wiederanstieg - 1974. Die Ergebnisse sind in Schaubild 24 dargestellt. ${ }^{199}$ Noch deutlicher als bei den bisherigen Resultaten zeigt sich, daß die eigentliche Krise nicht mit einem exzessiven Ausgabenanstieg zu Beginn der 70er Jahre begann, sondern mit der Rezession 1975. Zwar hätte eine Reduzierung des Schuldenstandes bzw. eine stärkere Verminderung der Schuldenquote die Situation in der Rezession erleichtert, die „Schuldenkrise“ hätte damit aber nicht vermieden werden können. Für die 80er Jahre erhält man mittelfristig insgesamt höhere Finanzlücken als für die 70er Jahre, die auch nur langsam abgebaut werden. Die Werte der mittelfristigen Primärüberschußlücken liegen zwischen 2 und 3,5\%. Die langfristigen Indikatoren zeigen eine deutlichere Reduzierung, bleiben aber ober-

199 Die langfristigen Indikatoren gehen wiederum bis einschließlich 1986. 
halb der 1\%-Marke. Wollte man also zu einer Situation vor der sozial-liberalen Koalition zurückkehren - setzte man also voraus, daß jede Regierung ihrer Nachfolgerin sozusagen den Haushalt in einem Zustand zu hinterlassen habe, in dem sie denselben vorgefunden hat -, müßte man in der Tat verminderte Konsolidierungschancen und mithin eine Erblast konstatieren. Denn wie die langfristigen Indikatoren andeuten, ist dies zwar nicht völlig unmöglich, aber mit erheblichen Einschränkungen verbunden und nur in einer sehr langen Frist zu bewältigen. Dies würde aber auch voraussetzen, daß die wirtschaftlichen Rahmenbedingungen unverändert bleiben müßten. Außerdem müßte unterstellt werden, daß es eine von der jeweiligen Regierung unabhängige, quasi von außen vorgegebene Zielsetzung des Staates gäbe. Geht man hingegen davon aus, da $B$ - und darum geht es letztlich bei den Tragfähigkeitsüberlegungen - die bereits erreichte Schuldenquote stabilisiert und damit eine Schuldenexplosion vermieden werden soll, dann ist festzustellen, $\mathrm{da} B$ es der christlich-liberalen Koalition gelungen ist - zumindest bis 1989 die Schuldenquote an dem erreichten Niveau zu halten und sogar teilweise zurückzuführen. Wie die Konsolidierungschancen eingeschätzt werden, hängt also damit letztlich auch davon ab, welche Schuldenquote stabilisiert werden soll. Die These, daß die sozial-liberale Finanzpolitik ein im Sinne der Tragfähigkeit vernünftiges Haushalten unmöglich gemacht hätte, ist damit widerlegt. Allerdings muß darauf hingewiesen werden, da $B$ dies nicht allein auf die Haushaltsführung der konservativ-liberalen Koalition zurückzuführen ist, sondern daß die Gewinnabführung der Bundesbank dazu einen nicht unerheblichen Beitrag geleistet hat, der in diesen Größenordnungen seit 1982 ein neues Phänomen ist. Darauf wird weiter unten im Zusammenhang mit Tabelle 7 noch einmal hingewiesen.

Die Frage der Zinslast bleibt gleichwohl erhalten, unabhängig vom Konsolidierungsziel. Denn sobald erst einmal ein Schuldenstand besteht, wird unweigerlich die Zinsdynamik in Kraft gesetzt, die lediglich durch Primärüberschüsse des Haushalts kompensiert werden kann. Allein ein dauerhaft negatives Zins-Wachstums-Differential kann (im Falle permanenter Defizite) ein kontinuierliches Wachstum der Schuldenquote verhindern. Dies heißt jedoch, daß unter realistischen Bedingungen (d.h. unter Bedingungen in denen das Zins-Wachstums-Diffferential nicht - zumindest nicht dauerhaft - kleiner Null ist) eine zusätzliche Kreditfinanzierung stets mit späteren Primärüberschüssen erkauft wird. Jede Regierung, die sich verschuldet, produziert 


\section{Schaubild 24}

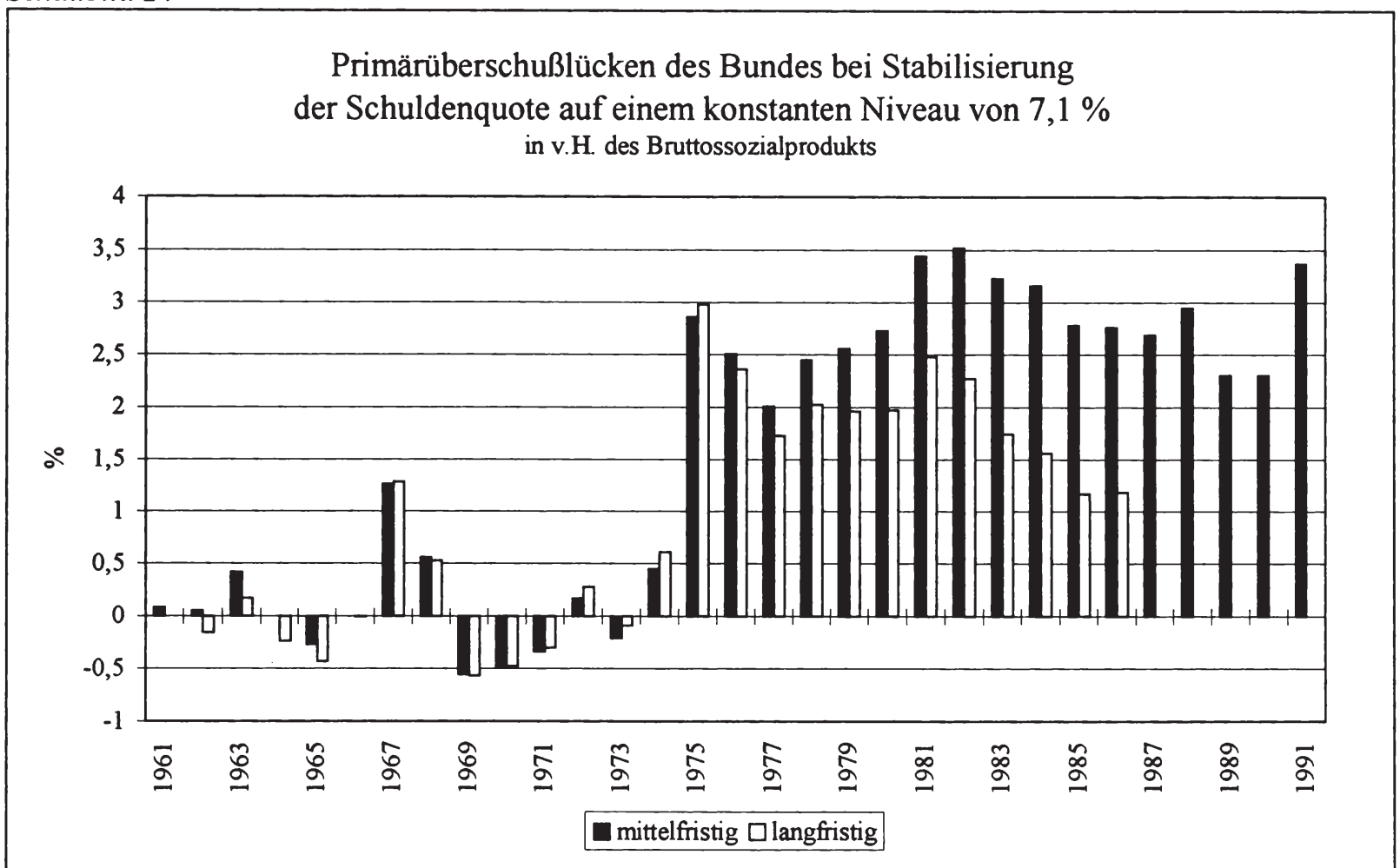

Anmerkung: Keine Angaben zu den langfristigen Werten der Jahre 1986 bis 1991 (siehe Text).

๑) Quelle: Statistisches Bundesamt (1994a, 1994b) sowie interne Unterlagen, Sachverständigenrat zur Begutachtung der gesamtwirtschaftlichen Entwicklung (1995); eigene Berechnungen. 
mithin eine Last, es sei denn, es gelingt ihr, das Wachstum derart anzuregen, daß hierüber die Schuldenquote stabilisiert wird. ${ }^{200}$ Dies ist aber nur unter Bedingungen möglich, die lediglich zeitlich begrenzt und eben nicht dauerhaft erfüllbar sind. Wird nun die notwendige Stabilisierung der Schuldenquote nicht von der sich verschuldenden Regierung selbst geleistet, dann müssen die entsprechenden Primärüberschüsse von einer späteren Regierung „erwirtschaftet" werden - einschließlich einer zusätzlichen Last, die sich infolge der zeitlichen Verzögerung aus der Zinsdynamik ergibt. ${ }^{201} \mathrm{Ob}$ diese Last auch eine Erblast darstellt in dem Sinne, daß damit eine Wohlfahrtsminderung einhergeht, ist im Rahmen der Lastverschiebungsdebatte eingehend, allerdings ohne eindeutiges Resultat diskutiert worden und soll hier nicht weiter thematisiert werden.

Es ist jedoch noch zu untersuchen, in welchem Maße der Ausgabenspielraum durch die Verschuldung eingeschränkt wurde. ${ }^{202}$ Hierzu wird auf eine einfache Beispielrechnung zurückgegriffen, mit Hilfe derer dargestellt werden kann, wie sich im Falle einer Kreditfinanzierung der Anteil der Zinsen an den Gesamtausgaben im Zeitablauf verändert und welcher Beitrag der Verschuldung damit letztlich noch zur Finanzierung originärer Ausgaben zur Verfügung steht. ${ }^{203}$ Zur Vereinfachung werden Defizitquote, Ausgabenquote, rechnerische Zins- und Wachstumsrate als konstant angenommen, wobei hier jeweils die Durchschnittswerte der Jahre 1970 bis 1982 zugrundegelegt werden, d.h. 1,6\% für die Defizitquote, $14,4 \%$ für die Ausgabenquote sowie $6,6 \%$ und 7,5\% für den rechnerischen Zins bzw. die Wachstumsrate. Das

200 Direkt, indem der Quotient aus Schuldenstand und Bruttosozialprodukt verringert wird und indirekt, indem die Steuereinnahmen steigen.

201 Blanchard hat im Falle von Einnahmenlücken gezeigt, daß die zur Stabilisierung notwendige Einnahmenquote proportional mit dem Zins-Wachstums-Differential und mit dem Zeitraum der Anpassungsverzögerung wächst. Beträgt die Einnahmenlücke z.B. $3 \%$, dann wächst sie bei einem Zins-Wachstums-Differential von $2 \%$ und einer Verzögerung von 5 Jahren um 0,3\%, vgl. Blanchard (1990), S. 14.

202 Daß der Handlungsspielraum auf der Ausgabenseite des Budgets neben den Zinsausgaben auch durch andere, kurzfristig nicht änderbare Ausgaben eingeengt wird, wurde bereits in Abschnitt 1.2 thematisiert. Die Ursachen sind jedoch nicht in einer erhöhten Verschuldung, sondern in rechtlichen, sozio-ökonomischen und anderen Faktoren zu suchen. Auf eine nähere Betrachtung wird daher verzichtet, vgl. hierzu Ewringmann (1975).

Vgl. Rürup (1982), insbesondere S. 617-626. 


\section{Schaubild 25}

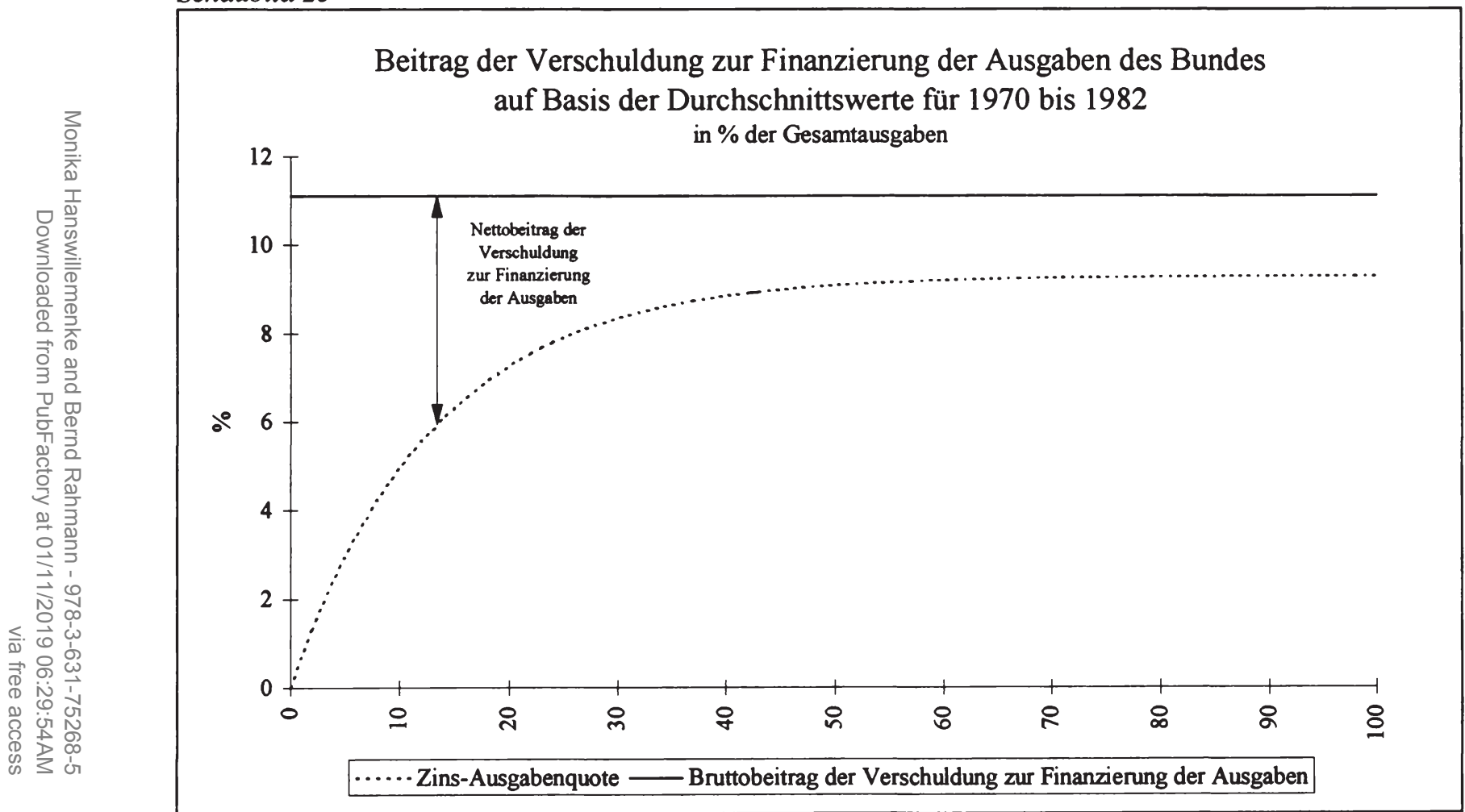

Quelle: Statistisches Bundesamt (1994a, 1994b) sowie interne Unterlagen, Sachverstăndigenrat zur Begutachtung der gesamtwirtschaftlichen Entwicklung (1995); eigene Berechnungen. 
Schaubild 26

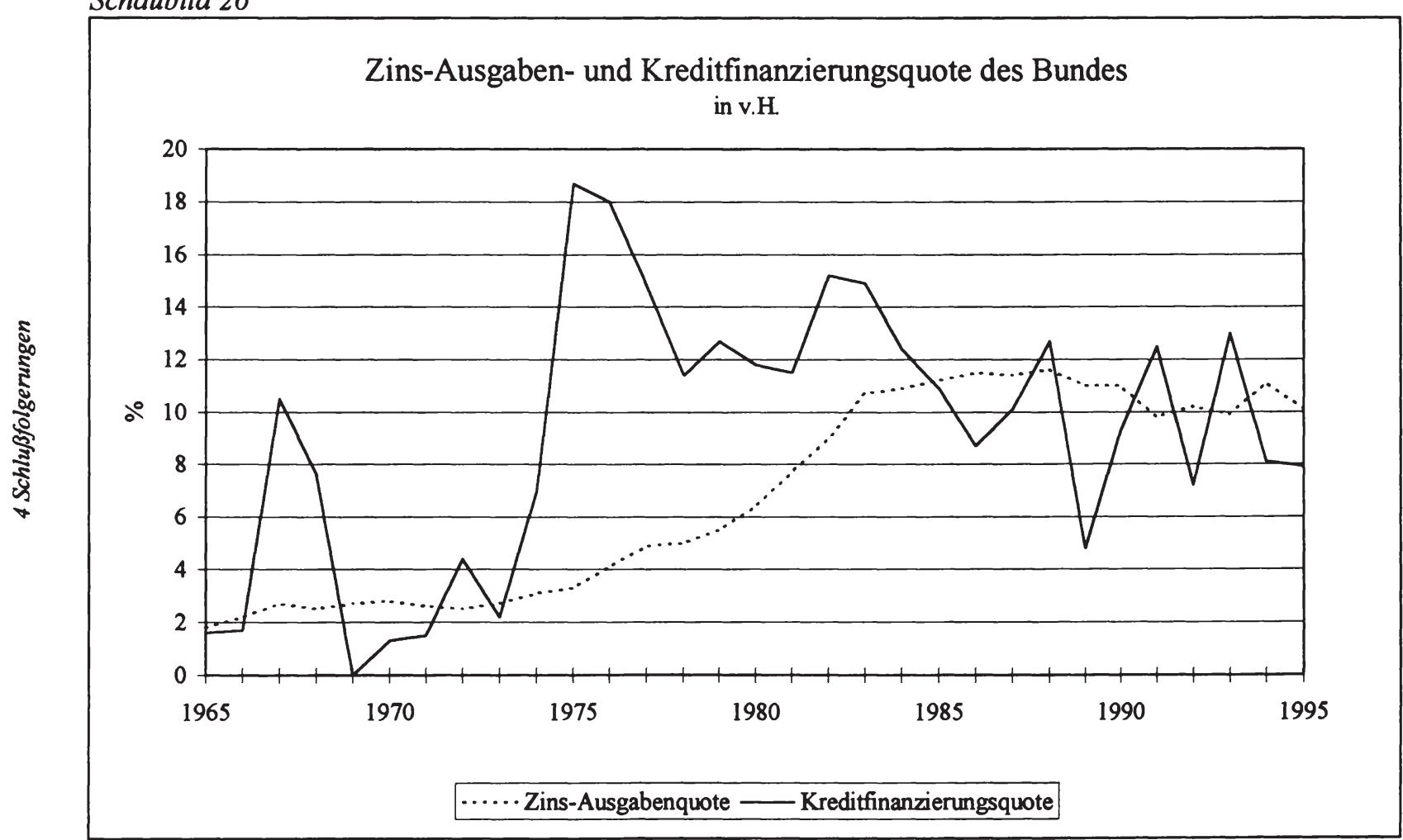


Ergebnis ist für einen Zeitraum von 100 Jahren in Schaubild 25 dargestellt worden. Insgesamt werden in dieser Rechnung 11,1\% der Ausgaben durch Kredite finanziert. Nach 12 Jahren muß bereits die Hälfte der aufgenommenen Mittel zur Finanzierung der Zinsausgaben herangezogen werden. Langfristig verbleiben lediglich rund $2 \%$ der Nettoneuverschuldung zur Finanzierung anderer Ausgaben. Wäre ein positives Zins-Wachstums-Differential angesetzt worden und nicht wie in den Beispielrechnungen ein negatives, dann hätte auf Dauer die Verschuldung nicht einmal zur Finanzierung der Zinsausgaben gereicht. ${ }^{204}$ Die tatsächlichen Werte sind zum Vergleich in Schaubild 26 abgebildet.

In der bisherigen Betrachtung wurden Schulden- und Haushaltsentwicklung jeweils in Relation zum Sozialprodukt gesetzt. Alternativ kann sie in Beziehung zu den regulären Einnahmen gesetzt werden. ${ }^{205}$ Die Entwicklung des Ausgabenspielraums, d.h. des Teils der Einnahmen (reguläre und Verschuldung), der nicht in Form von Zinsausgaben abfließt, hängt bei gegebenem Aufkommen der regulären Einnahmen vom Verhältnis der Nettokreditaufnahme und der Zinsausgaben ab. Liegt die Nettoneuverschuldung oberhalb der Zinsausgaben, dann wird der Ausgabenspielraum ausgeweitet, liegt sie unterhalb, wird er entsprechend eingeengt. Die Erhaltung des Ausgabenspielraums ist bei unveränderter Steuerpolitik nur bei fortgesetzter Verschuldung möglich, und zwar muß dazu das Wachstum des Schuldenstandes mindestens so groß sein wie das seiner Verzinsung. ${ }^{206}$

Setzt man nun den Schuldenstand der Nettoneukreditaufnahme und der Zinsausgaben in Relation zu den regulären Einnahmen, dann zeigt sich folgendes: In dem Fall, daß der (rechnerische) Zins oberhalb des Einnahmenwachstums liegt, kann der Ausgabenspielraum nur bei einem Anstieg des Schuldenstandsquotienten (Schuldenstand in v.H. der regulären Einnahmen) gewahrt werden. Lediglich in dem Fall, daß die Verzinsung kleiner als das Einnahmenwachstum ist bzw. diesem gerade entspricht, ist die Wahrnehmung des Ausgabenspielraums ohne einen zwangsläufigen Anstieg des

\footnotetext{
204 In diesem Fall hätte die Kurve der Zins-Ausgabenquote die Bruttobeitrags-Gerade geschnitten.

205

206

Vgl. Gschwendter (1981).

Vgl. Gschwendter (1981), S. 309.
} 
Schuldenstandsquotienten möglich. ${ }^{207}$ Die tatsächlichen Wachstumsraten der laufenden Einnahmen sowie die Verzinsung der Schulden sind für den Bund in Schaubild 27 wiedergegeben. Parallel zur Wachstumsrate zeigt sich ein insgesamt sinkender Trend, was die Abhängigkeit der Einnahmen von der gesamtwirtschaftlichen Entwicklung widerspiegelt. Hierbei zeigt sich, daß zu Beginn der 70er Jahre sowie von 1977 bis Ende der 70er Jahre das Einnahmenwachstum oberhalb der Verzinsung liegt, von 1981 bis 1989 das Einnahmenwachstum permanent und mit sinkender Tendenz darunter bleibt. Darüber hinaus weist der rechnerische Zins selbst eine steigende Tendenz auf.

Aus dieser Entwicklung lassen sich im Zusammenhang mit dem Schuldenstandsquotienten folgende Schlüsse ziehen: Während die sozial-liberale Koalition die Haushaltssituation teilweise zu einer Reduzierung des Schuldenstandsquotienten genutzt hat (1970 bis 1973), teilweise aber den Ausgabenspielraum trotz über dem Zins liegenden Wachstums der Einnahmen zu Lasten des Schuldenstandsquotienten ausgeweitet hat (1970; 1977 bis 1980), bestand für die christlich-liberale Koalition sozusagen ein trade-off zwischen der Stabilität des Schuldenstandsquotienten und der Bewahrung des Ausgabenspielraums, d.h. das eine Ziel konnte nur auf Kosten des jeweils anderen verwirklicht werden. Dieser Zielkonflikt wird durch Steuererleichterungen im Zuge der stärkeren Angebotsorientierung von der konservativ-liberalen Koalition selbst noch verstärkt. ${ }^{208}$ Dies wurde auch bereits an der Entwicklung der Ausgabenlücken deutlich. Zusammenfassend läßt sich feststellen, daß der fiskalische Handlungsspielraum der konservativ-liberalen Koalition zu einem gewissen Teil durch die Verschuldung seit Mitte der 70er Jahre eingeengt wurde, zum Teil jedoch auch hausgemacht war.

207 Indem nun die Schulden sowie Nettokreditaufnahme und Zinsausgaben in Relation zu den laufenden Einnahmen gesetzt werden, statt wie bisher in Relation zum (nominalen) Bruttosozialprodukt, wird anstelle des Zins-Wachstums-Differentials die Differenz zwischen dem rechnerischen Zins und dem Einnahmenwachstum für das Wachstum des Schuldenstandsquotienten zur relevanten Größe.

Einschränkend sei jedoch erwähnt, daß weder die inflationsbedingten realen Ausgabeneinschränkungen berücksichtigt sind, noch daß in einer wachsenden Wirtschaft auch die staatlichen Ausgaben steigen müssen. Letzteres fließt nur insofern mit ein, als in einer wachsenden Wirtschaft (unter sonst gleichen Bedingungen) auch die regulären Einnahmen wachsen. 


\section{Schaubild 27}

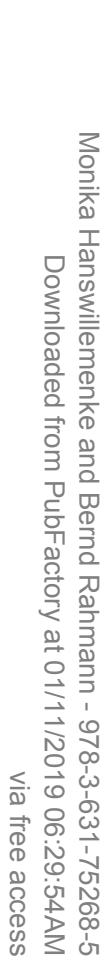

\section{Wachstumsrate der Einnahmen und rechnerischer Zinssatz}

für den Bund

in v.H.

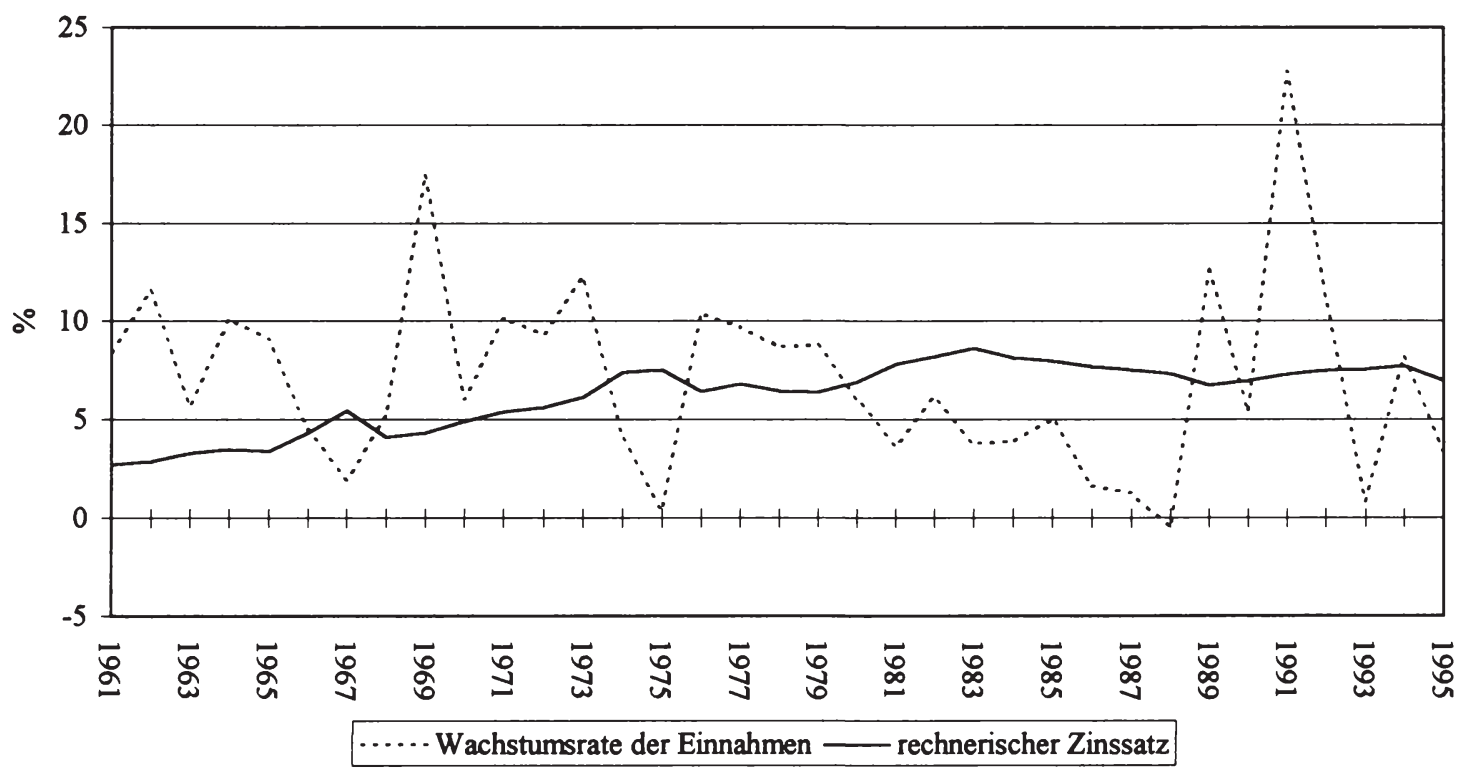

Anmerkung: Die Wachstumsrate 1991 ist mit den Vorjahren nur begrenzt vergleichbar, da der Bund für das erweiterte Bundesgebiet zuständig ist. Quelle: Statistisches Bundesamt (1994a, 1994b, 1995) sowie interne Unterlagen; eigene Berechnungen. 
Hier wird ein allgemeines, zumindest kurzfristiges Dilemma sichtbar: Sobald eine öffentliche Verschuldung existiert und somit der beschriebene ZinsWachstums-Differential-Mechanismus aktiviert wird, ist eine Hochzinspolitik, die u.a. zur Bekämpfung inflationärer Tendenzen eingesetzt wird, im Falle des Erfolgs, also Senkung der Inflationsrate, zugleich eine Gefahr für den öffentlichen Haushalt, wenn die Zinssätze von der Zentralnotenbank nicht rasch abgesenkt werden. Denn dadurch steigt die Wahrscheinlichkeit, daß die Differenz zwischen Zinssatz und nominaler Wachstumsrate größer wird. Die realen Wachstumsraten lassen sich eben nicht beliebig steigern.

Vergleicht man die Zeiträume 1970 bis 1982 und 1983 bis 1989, indem man das Wachstum der Schuldenquote in seine Komponenten zerlegt (vgl. Tabelle 7), dann ergibt sich folgendes Bild: Es zeigt sich, daß rund vier Fünftel des Anstiegs der Schuldenquote auf die Periode 1970 bis 1982 zurückgehen, ein Fünftel auf die Jahre 1983 bis 1989. Umgelegt auf die einzelnen Jahre erhält man einen Anteil von jeweils 6,6 bzw. 3,1\%, d.h. die Jahre 1970 bis 1982 hatten einen durchschnittlich doppelt so großen Anteil am Anstieg der Schuldenquote wie die Jahre 1983 bis 1989. Dafür verantwortlich sind in erster Linie die durchschnittlich höheren Primärdefizite (unter Herausrechnung des Bundesbankgewinns). Diese führen im Durchschnitt 1970 bis 1982 zu einem Anstieg der Schuldenquote von 7,4\% pro Jahr; 1983 bis 1989 liegt ihr Anteil bei durchschnittlich 2,9\% pro Jahr. Der Anteil der Zinsausgaben wird in den Jahren 1970 bis 1982 leicht durch das Wachstum des Bruttosozialprodukts überkompensiert. In den Jahren 1983 bis 1989 ist dies aufgrund gestiegener Zinsen sowie eines geringeren Wachstums nicht mehr gegeben. Einen deutlichen Unterschied gibt es zudem, wie bereits erwähnt, in der Rolle, die der Bundesbankgewinn bei dieser Entwicklung spielt. 1970 bis 1982 trägt er lediglich mit durchschnittlich 0,5\% zur Begrenzung des Wachstums der Schuldenquote bei, 1983 bis 1989 mit durchschnittlich 3,7\%. Daß die Bundesregierung Primärüberschüsse erzielen konnte, ist damit zu einem nicht unerheblichen Teil durch den Zufluß des Bundesbankgewinns ermöglicht worden. Der Haushalt selbst blieb defizitär.

Gerade die Position Bundesbankgewinn zeigt, daß eine Berechnung, wie sie in Tabelle 7 abgebildet ist, zwar ex post nicht mehr korrigiert werden kann, jedoch gegenüber Veränderungen einzelner Positionen theoretisch sehr sensibel reagiert, weil die betrachteten Komponenten nicht unabhängig vonein- 
ander sind. Hätte bereits der sozial-liberalen Regierung ein ähnlich hoher Bundesbankgewinn zur Verfügung gestanden, dann wären infolge dieser zusätzlichen Finanzierungsquelle das Defizit und damit die Zinslast geringer ausgefallen. Dies hätte zweifach in die gleiche Richtung auf die Schuldenquote gewirkt und deren Verteilung auf die betrachteten Zeiträume wesentlich ausgeglichener gestaltet.

Tabelle 7: Komponenten der Veränderung der Schuldenquote 1970-1989

\begin{tabular}{|c|c|c|c|c|c|}
\hline \multicolumn{7}{|c|}{ Anteil an der Veränderung der Schuldenquote } \\
in v.H.
\end{tabular}

Anmerkung: (a) Primărdefizit ohne Bundesbankgewinn. (b) Die Summe der Anteile von Primărdefizit, Zinsausgaben etc. über beide Zeiträume ergibt $108 \%$. Die Differenz zu $100 \%$ erklärt sich aus der nicht vollstăndigen Kompatibilităt der Haushalts- und der Schuldenstatistik.

Quelle: Statistisches Bundesamt (1994b) sowie interne Unterlagen, Sachverständigenrat zur Begutachtung der gesamtwirtschaftlichen Entwicklung (1995), Deutsche Bundesbank, Geschăftsbericht, diverse Jahrgănge; eigene Berechnungen.

Bestätigt sich somit die These von einer Erblast durch Staatsverschuldung? Unter einem isolierten und rein fiskalischen Aspekt ist die Antwort zunächst scheinbar eindeutig: ja! Im Falle eines streng objektbezogenen Defizits, bei dem die Erträge des schuldfinanzierten Objektes direkt die Zinszahlungen und die Abschreibungen decken und die Tilgungen daraus prompt erfolgen, trägt sich die Schuldfinanzierung allerdings selbst, und der gesamte Vorgang läuft quasi völlig unabhängig vom übrigen Budget $a b$. Die Tragfähigkeit ist isoliert und gewissermaßen durch das Objekt selbst gesichert. Abgesehen von den nur schwer lösbaren Problemen einer exakten Zurechenbarkeit ist dieser Fall auch sonst nicht die Regel, weil im Sinne einer intertemporalen Effizienz und dem Prinzip des „pay as you use“ mittels öffentlicher Verschuldung ausdrücklich eine Belastung der Zukunft stattfinden soll. Bezogen auf die Zahlungsabläufe, also rein zahlungstechnisch, gelingt diese Bela- 
stung auch immer, denn die gegenwärtige Schuld erfordert zukünftige Steuern für ihre Bedienung.

Mit eingeengtem Blick auf diesen rein fiskalischen Aspekt ist ein umfassend gemeinter politischer Vorwurf der Erblast also trivial, weil er so stets zutrifft. Schließlich kann der eng an ein Objekt gebundene Gedanke einer sich selbst tragenden (self-liquidating) Verschuldung auch auf die antizyklische Schuldenfinanzierung angewendet werden, wobei als Objekt quasi die Gesamtwirtschaft fungiert und die Erträge dieses Objektes in Form von steuerlichen Rückflüssen entstehen. Erfolgreiches Deficit Spending und die Finanzierung des Schuldendienstes sowie der Tilgung aus eben diesen Erfolgen, die ohne öffentliche Verschuldung nicht eingetreten wären, führen dann ebenfalls dazu, daß die Zukunft auch fiskalisch nicht belastet (im besten Fall sogar noch begünstigt) wird. ${ }^{209}$ Es läßt sich jedenfalls nicht stichhaltig begründen, daß die nachfolgende Haushalts- und Finanzpolitik gefesselt wird und keinen eigenen Entscheidungsspielraum mehr besitzt. So zeigt sich dies denn auch empirisch nach 1982 nicht, denn es hat ja faktisch eine Wende stattgefunden, indem konsolidiert und die Staatsquote reduziert wurde. Auch bei der politischen Zielsetzung ist der Übergang von einem umfassend definierten gesamtwirtschaftlichen Stabilisierungsziel zu einer eng gefaßten Interpretation und Konzentration auf Preisniveaustabilität konsequenterweise vollständig gelungen. Die politische Entwicklung seit 1990 schließlich läßt sich schon gar nicht unter dem Aspekt von Restriktionen aus der Vergangenheit beurteilen, weil gänzlich neue Rahmenbedingungen eingetreten sind.

Auch methodisch ist die auf die spezifische Inzidenz gerichtete Partialanalyse eines isolierten Finanzierungsinstruments, so wichtig sie für die Erarbeitung partieller Wirkungszusammenhänge auch ist, unzureichend für eine qualitative Beurteilung von Politik. In einem weiteren Schritt muß nämlich gefragt werden, welche Effekte zu erwarten gewesen wären, wenn anstelle der Schuldfinanzierung eine Steuerfinanzierung getreten wäre, wenn also die

209 Oberhauser (1985, S. 333ff.) nennt diesen Fall das „Schuldenparadox“, weil öffentliche Verschuldung in einer Rezession bei unterstellten positiven Rückwirkungen auf das Staatsbudget nicht zu steigendem, sondern nach Überwindung der Rezession zu sinkendem Schuldenstand führt. Dies ist allerdings an eine Reihe von politischen, institutionellen und funktionalen Bedingungen geknüpft, die realiter nicht ohne weiteres stets erfült sind. 
differentielle Inzidenz untersucht werden würde. Dieser Ansatz ist aus der Analyse der personellen und intertemporalen Wirkungen der öffentlichen Verschuldung gut bekannt: Der durch die gegenwärtige Aufnahme von öffentlichen Schulden in Zukunft erhöhten Steuerlast für Verzinsung und Tilgung müssen diejenigen Wirkungen gegenübergestellt werden, die in der Gegenwart durch Verzicht auf Steuererhöhung (oder durch Steuersenkung) entstehen. Diese Frage kann auch so formuliert werden, daß sie auf die Wirkungen einer gegenwärtigen Steuererhöhung gerichtet ist, durch die eine Ausdehnung der öffentlichen Verschuldung und somit zukünftige Lasten daraus vermieden werden können. Wäre daraus keine Last für die Zukunft entstanden? Hätte dies den finanzpolitischen Handlungsspielraum nicht eingeengt oder sogar noch erhöht?

Diese Perspektive ist hypothetisch; tendenziell lassen sich jedoch Hinweise formulieren: Zum einen hätten Steuererhöhungen sicherlich distributive Wirkungen hervorgebracht, die der herrschenden politischen Linie entsprochen hätten. Das ist selbstverständlich für demokratisch gewählte Regierungen mit eigenständigen Zielen, und ebenso selbstverständlich ist es, daß die politische Einfärbung auf Kritik der jeweiligen Opposition stößt. Bei einem Regierungswechsel findet dann natürlich auch eine Veränderung der verteilungspolitischen Perspektive in der Besteuerung statt. Politische Erblast ist dies selbstverständlich auch, weil es eine politische Veränderung insgesamt gegeben hat. Aber daraus einen ernsthaften Vorwurf zu machen, ist offensichtlich fehl am Platz; es handelt sich schlicht um eine andere politische Programmatik, und die abgelöste Regierung wird mit der Kritik sicherlich leben können.

Zum anderen hätten Steuererhöhungen den privaten Konsum oder die Ersparnis oder beides zurückgedrängt. Die davon zu erwartenden Wirkungen auf den Auslastungsgrad und das Wachstum des Produktionspotentials sind nur unter ganz bestimmten Bedingungen neutral. In aller Regel sind Steuererhöhungen bei hoher bzw. wachsender Arbeitslosigkeit jedenfalls kein angemessenes Instrument, und die Vermutung, daß bei Ersatz der Schuldfinanzierung durch gleich hohe Steuerfinanzierung in den 70er Jahren eine wesentlich höhere Arbeitslosigkeit entstanden wäre, ist durchaus plausibel. Ganz sicher wären die dann entstandenen Mehrkosten einer höheren Arbeitslosigkeit als eine Erblast für die 80er Jahre zu bezeichnen gewesen, de- 
ren tatsächliche Vermeidung folglich mit der konstatierten fiskalischen Erblast zu vergleichen und politisch zu bewerten wäre. Es wurde ja gerade deshalb gezeigt, in welchem Ausmaß die Haushalts- und Finanzpolitik durch die Bemühungen der sozial-liberalen Koalition um Wiedererlangung der Vollbeschäftigung belastet gewesen ist. $\mathrm{Daß}$ diese Bemühungen wegen der teils neuartigen Problemlage auf dem Arbeitsmarkt nicht zu größerem Erfolg geführt haben, ist sicherlich kritikwürdig; nicht zuletzt dafür hat ja auch ein Regierungswechsel stattgefunden. Eine Erblast wäre daraus aber erst abzuleiten, wenn jegliche Bemühungen unterblieben und die der Zukunft überlassene Arbeitslosigkeit mit all ihren gesellschaftlichen und fiskalischen Folgen noch höher ausgefallen wäre.

Schließlich muß im umfassendsten Sinne die Analyse auf das gesamte Budget ausgedehnt werden. D.h. es geht nicht darum, nur die öffentliche Verschuldung durch Besteuerung zu ersetzen, sondern darum, die öffentlichen Ausgaben zurückzudrängen, damit weder die eine noch die andere Finanzierungsart Probleme aufwerfen kann. Faktisch steht damit gar nicht die Art der öffentlichen Finanzierung, sondern deren Höhe und die damit erreichten Zwecke zur Diskussion. Beschäftigungspolitisch muß konstatiert werden, daß eine Absenkung der öffentlichen Ausgaben wegen geringerer Steuereinnahmen in einer Rezession der klassische Fall der Parallelpolitik ist. Massenarbeitslosigkeit als Folge daraus ist nicht nur historisch in Deutschland nachdrücklich belegbar, sondern makroökonomisch auch theoretisch gut formulierbar. ${ }^{210}$ Die entsprechenden Kreislaufzusammenhänge können einfach nicht vernachlässigt bzw. geleugnet werden.

Gerade auch für die heute in der Bundesrepublik gegebene Situation sind derartige Überlegungen relevant. Würde allein die seit der deutschen Vereinigung enorm gestiegene öffentliche Verschuldung zum Maßstab für die Politik der letzten fünf Jahre gemacht, ergäbe sich ein ebenso eindeutiges wie unvollständiges Bild. Die fiskalischen Kosten für die Zukunft sind in der Tat bedrohlich hoch und die zu einer Konsolidierung erforderlichen Haushaltsüberschüsse nach heute gängigen Vorstellungen kaum vorstellbar. Was wäre aber geschehen, wenn die öffentlichen Lasten der deutschen Vereinigung ausschließlich über Steuererhöhungen finanziert worden wären? Oder wie

210 Siehe zu dieser Argumentation für die Gegenwart Oberhauser (1996, S. 566ff.). 
wäre die Situation einzuschätzen, wenn durch den Staat wesentlich geringere oder gar keine Lasten übernommen und die Entwicklungen einer rein privatwirtschaftlichen Dynamik überlassen worden wären? Mit einiger Sicherheit kann prognostiziert werden, daß in diesem hypothetischen Fall die Lage in den neuen Bundesländern bzw. in der gesamten Bundesrepublik Deutschland sowohl ökonomisch als auch sozial schlechter wäre als sie es tatsächlich ist. $\mathrm{Ob}$ die amtierende Regierung dies politisch überstanden hätte, mag hier dahingestellt bleiben. Die einseitig fiskalische Perspektive ohne Berücksichtigung einer umfassenden Budgetinzidenz ist schließlich auch ein Mangel der gegenwärtigen Verengung der Sichtweise für die Kriterien zum Beitritt zur Europäischen Währungsunion. Allein die relative Höhe der öffentlichen Verschuldung ist ein schlechter Indikator für die Leistungsfähigkeit einer Volkswirtschaft, deren sozio-ökonomische Tragfähigkeit bei wachsender Arbeitslosigkeit und brüchiger werdenden gesellschaftlichen Fundamenten in Gefahr gerät.

Erst wenn nachgewiesen werden kann, daß eine Regierung ineffizient, also verschwenderisch, gewirtschaftet hat, so wäre die daraus entstandene Finanzierungslast (egal in welcher Form) eine Erblast zu nennen. Jenseits einer solchen reinen, ohne jede Wirkung auf die intendierten Ziele gekennzeichneten Vergeudung von Ressourcen ist eine objektive Beurteilung der erreichten Staatszwecke ohne Bestimmung der Beurteilungskriterien aber bekanntlich nicht möglich. Subjektive und parteipolitisch definierte Kriterien sind nicht vergleichbar im Sinne eines allgemeinen Urteils. Fehlen aber objektive Kriterien, so ist über unterschiedlich hohe Staatsanteile wiederum nur ein parteipolitisches Urteil möglich. In diesem Sinne ist Erblast dann zwar ein probates Mittel, um den politischen Gegner zu diskriminieren. In einer ökonomisch-analytisch angelegten Untersuchung sollte dagegen dieser Begriff nicht verwendet werden. 
Monika Hanswillemenke and Bernd Rahmann - 978-3-631-75268-5

Downloaded from PubFactory at 01/11/2019 06:29:54AM

via free access 


\section{Literatur}

Amann, E./Jäger, A. (1989): Staatsschuldenarithmetik: Zwei unerfreuliche Beispiele, in: Kredit und Kapital, S. 221-234.

Aschauer, D.A. (1989): Is Public Expenditure Productive?, in: Journal of Monetary Economics, Vol. 23, S. 177-200.

Barker, T. (1985): Erfahrungen mit dem Monetarismus in Großbritannien, in: Langkau, J./Köhler, C. (Hrsg.), Wirtschaftspolitik und wirtschaftliche Entwicklung. Festschrift für Walter Hesselbach, Bonn.

Barro, R.J./Sala-I-Martin, X. (1995): Economic Growth, New York u.a.

Benassi, C./Chirco, A./Colombo, C. (1994): The New Keynesian Economics, Oxford.

Berthold, N. (1988): Keynesianische versus klassische Arbeitslosigkeit, in: WiSt, 17. Jg., Heft 10, Oktober 1988, S. 485-493.

Beyfuss, J. (1977): 10 Jahre Stabilitätsgesetz. Erfahrungen und Kritik, Beiträge zur Wirtschafts- und Sozialpolitik Nr. 45, Institut der deutschen Wirtschaft.

Biehler, H./Brandes, W./Buttler, F./Gerlach, K./Liepmann, P. (1981): Arbeitsmarktstrukturen und -prozesse, Tübingen.

Blanchard, O. (1990): Suggestions For A New Set Of Fiscal Indicators, OECD Working Papers, No. 79, April 1990.

Blanchard, O./Chouraqui, J.-Cl./Hagemann, R.P./Sartor, N. (1990): The Sustainability Of Fiscal Policy: New Answers To An Old Question, OECD Economic Studies, No. 15, S. 7-36.

Blinder, A.M./Solow, R.M. (1973): Does Fiscal Policy Matter?, in: Journal of Public Economics, Vol. 2, S. 319-337.

Bolle, M. (1983): Anpassungsprozesse auf Arbeitsmärkten bei Gütermarktimpulsen, in Bolle, M./Gabriel, J. (Hrsg.), Die Dynamik der Arbeitsmärkte aus der Sicht internationaler Forschung, München.

Bolle, M./Gabriel, J. (Hrsg.)(1983): Die Dynamik der Arbeitsmärkte aus der Sicht internationaler Forschung, München.

Bombach, G./Netzband, K.-B./Ramser, H.-J./Timmermann, M. (Hrsg.) (1983): Der Keynesianismus, Band IV. Die beschäftigungspolitische Diskussion in der Wachstumsepoche der Bundesrepublik Deutschland, Berlin u.a., S. 1f. 
Boss, A./Lorz, J.O. (1995): Die öffentliche Verschuldung in der Bundesrepublik Deutschland. Ausmaß und Belastungswirkung, Kieler Arbeitspapier Nr. 669 (Institut für Weltwirtschaft an der Universität Kiel).

Brandt, W. (1969): Im Bewußtsein der Verantwortung für die Zukunft unseres Landes. Regierungserklärung des Bundeskanzlers vor dem Deutschen Bundestag am 28. Oktober 1969, abgedruckt in: Bulletin Nr. 132, S. 1121-1128.

Bruche, G./Reissert, B. (1985): Die Finanzierung der Arbeitsmarktpolitik. System, Effektivität, Reformansätze, Arbeitsberichte des Wissenschaftszentrums Berlin - Internationales Institut für Management und Verwaltung/Arbeitsmarktpolitik, Frankfurt/Main, New York.

Buiter, W.H. (1985): A guide to public sector debt and deficits, in: Economic Policy, No. 1, S. 14-79.

Bundesministerium der Finanzen (1967): Finanzbericht 1968, Bonn.

Bundesministerium der Finanzen (1968): Finanzbericht 1969, Bonn.

Bundesministerium der Finanzen (1969): Finanzbericht 1970, Bonn.

Bundesministerium der Finanzen (1970): Finanzbericht 1971, Bonn.

Bundesministerium der Finanzen (1971): Finanzbericht 1972, Bonn.

Bundesministerium der Finanzen (1972): Finanzbericht 1973, Bonn.

Bundesministerium der Finanzen (1973): Finanzbericht 1974, Bonn.

Bundesministerium der Finanzen (1974): Finanzbericht 1975, Bonn.

Bundesministerium der Finanzen (1975): Finanzbericht 1976, Bonn.

Bundesministerium der Finanzen (1976): Finanzbericht 1977, Bonn.

Bundesministerium der Finanzen (1977): Finanzbericht 1978, Bonn.

Bundesministerium der Finanzen (1978): Finanzbericht 1979, Bonn.

Bundesministerium der Finanzen (1979): Finanzbericht 1980, Bonn.

Bundesministerium der Finanzen (1980): Finanzbericht 1981, Bonn.

Bundesministerium der Finanzen (1981): Finanzbericht 1982, Bonn.

Bundesministerium der Finanzen (1982): Finanzbericht 1983, Bonn.

Bundesministerium der Finanzen (1983): Finanzbericht 1984, Bonn.

Bundesministerium der Finanzen (1995): Finanzbericht 1996, Bonn.

Bundesministerium der Finanzen (1996): Finanzbericht 1997, Bonn.

Buti, M. (1990): Monetary and Fiscal Rules for Public Debt Sustainability, Economic Papers No. 84, ed. by the Commission of the European Communities, September 1990.

Buttler, F./Kühl, J./Rahmann, B. (Hrsg.)(1985): Staat und Beschäftigung. Angebots- und Nachfragepolitik in Theorie und Praxis, Beiträge zur Ar- 
beitsmarkt- und Berufsforschung 88, (Institut für Arbeitsmarkt- und Berufsforschung der Bundesanstalt für Arbeit), Nürnberg.

Caesar, R. (1988): Öffentliche Verschuldung in Deutschland seit der Weltwirtschaftskrise: Wandlungen in Politik und Theorie, Ruhr-Universität Bochum, Seminar für Wirtschafts- und Finanzpolitik, Diskussionsbeitrag Nr. 5.

Chouraqui, J.-Cl./Hagemann, R.P./Sartor, N. (1990): Indicators Of Fiscal Policy: A Reassessment, OECD Working Papers, No. 78, April 1990.

Coen, R.M./Hickman, B.G. (1987): Keynesian and Classical Unemployment in Four Countries, in: Brookings Papers on Economic Activity, 1:1987, S. 123-193.

Coen, R.M./Hickman, B.G. (1988): Is European Unemployment Classical or Keynesian?, in: American Economic Review, Vol. 78, S. 188-193.

Conrad, K./Seitz, H. (1994): The economic benefits of public infrastructure, in: Applied Economics, Vol. 26, S. 303-311.

Cornelius, P. (1990): Münzgewinne und Haushaltsfinanzierung in der offenen Volkswirtschaft, in: WiSt, Heft 12, Dezember 1990, S. 615-616.

Crihfield, J.B./Panggabean, M.P.H. (1995): Is public infrastructure productive? A metropolitan perspective using new capital stock estimates, in: Regional Science and Urban Economics, Vol. 25, S. 607-630.

Deutsche Bundesbank (1969): Geschäftsbericht für das Jahr 1969. Deutsche Bundesbank (1970): Geschäftsbericht für das Jahr 1970. Deutsche Bundesbank (1971): Geschäftsbericht für das Jahr 1971. Deutsche Bundesbank (1972a): Geschäftsbericht für das Jahr 1972.

Deutsche Bundesbank (1972b): Die Entwicklung der öffentlichen Investitionsausgaben seit 1961, in: Monatsberichte der Deutschen Bundesbank, 24. Jg., Nr. 4, S. 12-20.

Deutsche Bundesbank (1973a): Geschäftsbericht für das Jahr 1973.

Deutsche Bundesbank (1973b): Die Gebietskörperschaften im Konjunkturverlauf seit 1967, in: Monatsberichte der Deutschen Bundesbank, 25. Jg., Nr. 11, S. 12-28.

Deutsche Bundesbank (1974): Geschäftsbericht für das Jahr 1974.

Deutsche Bundesbank (1975): Geschäftsbericht für das Jahr 1975.

Deutsche Bundesbank (1976): Geschäftsbericht für das Jahr 1976.

Deutsche Bundesbank (1977): Geschäftsbericht für das Jahr 1977.

Deutsche Bundesbank (1978a): Geschäftsbericht für das Jahr 1978. 
Deutsche Bundesbank (1978b): Die Entwicklung der Steuerbelastung seit Beginn der siebziger Jahre, in: Monatsberichte der Deutschen Bundesbank, 30. Jg., Nr. 11, S. 21-28.

Deutsche Bundesbank (1979a): Geschäftsbericht für das Jahr 1979.

Deutsche Bundesbank (1979b): Die Verschuldung der öffentlichen Haushalte, in: Monatsberichte der Deutschen Bundesbank, 31. Jg., Nr. 7, S. 1522.

Deutsche Bundesbank (1980): Geschäftsbericht für das Jahr 1980.

Deutsche Bundesbank (1981a): Geschäftsbericht für das Jahr 1981.

Deutsche Bundesbank (1981b): Die Ausgaben der Gebietskörperschaften seit Mitte der siebziger Jahre, in: Monatsberichte der Deutschen Bundesbank, 33. Jg., Nr. 1, S. 20-27.

Deutsche Bundesbank (1982): Geschäftsbericht für das Jahr 1982.

Deutsche Bundesbank (1984): Sachinvestitionen, Darlehen und sonstige Finanzierungshilfen der Gebietskörperschaften seit Mitte der siebziger Jahre, in: Monatsberichte der Deutschen Bundesbank, 36. Jg., Nr. 11, S. 2634.

Deutsche Bundesbank (1988): Entwicklung und Struktur der Ausgaben der Gebietskörperschaften für Sachinvestitionen, in: Monatsberichte der Deutschen Bundesbank, 40 Jg., Nr. 8, S. 32-39.

Deutsche Bundesbank (1997): Monatsbericht Mai, Frankfurt/Main.

Diekheuer, G. (1978): Staatsverschuldung und wirtschaftliche Stabilisierung, Baden-Baden.

Domar, E.D. (1944): The "Burden of the Debt" and the National Income, in: American Economic Review, Vol. 34, S. 798-827, reprinted in: The American Economic Association (Hrsg.): Readings in Fiscal Policy, London 1971, S. 479-501.

Drèze, J./Bean, Ch. (1990): European Unemployment: Lessons from a Multicountry Econometric Study, in: Scandinavian Journal of Economics, Vol. 92, S. 135-165.

Duwendag, D. (1991): Zur Frage eines tragfähigen Policy mix: Sind adäquate Regeln für die Fiskalpolitik unentbehrlich?, in: Weber, M. (Hrsg.): Europa auf dem Weg zur Währungsunion, Darmstadt, S. 220-248.

Eckey, H.-F. (1978): Strukturorientierte Konjunkturpolitik, Köln.

Elsner, H. (1978): Gemeindehaushalte, Konjunktur und Finanzausgleich, Baden-Baden. 
Elsner, H./Schüler, M. (1970): Das Gemeindefinanzreformgesetz. Einführung in die Gemeindefinanzreform und Erläuterungen zum Gemeindefinanzreformgesetz, Hannover.

Entorf, H. (1995): Mismatch-Arbeitslosigkeit in Deutschland: Eine Kritik bestehender Meßkonzepte und neue Evidenz, in: Steiner, V./Bellmann, L. (Hrsg.): Mikroökonomik des Arbeitsmarktes, Beiträge zur Arbeitsmarktund Berufsforschung 192 (Institut für Arbeitsmarkt- und Berufsforschung der Bundesanstalt für Arbeit), Nürnberg, S. 31-49.

Entorf, H./Franz, W./König, H./Smolny, W. (1989): The Development of German Employment and Unemployment: Estimation and Simulation of a Disequilibrium Macro Model, Discussion Paper No. 384-89, Universität Mannheim.

Ernst-Pörksen, M. (1983): Staatsschuldtheorien. Vom Merkantilismus bis zur gegenwärtigen Kontroverse um die Staatsverschuldung, Berlin.

Evans, P. (1987): Do Budget Deficits Raise Nominal Interest Rates? Evidence From Six Countries, Journal of Monetary Economics, Vol. 20, S. 281-300.

Ewringmann, D. (1975): Die Flexibilität öffentlicher Ausgaben, Göttingen.

Fabritius, H.G. (1975): Konjunkturtheoretische Vorstellungen des Sachverständigenrates zur Begutachtung der gesamtwirtschaftlichen Entwicklung. Eine Analyse der bis einschließlich 1972 veröffentlichten Gutachten, Berlin.

Fazzari, St.M. (1994): Why doubt the effectiveness of Keynesian fiscal policy?, in: Journal of Post Keynesian Economics, Vol. 17, No. 2, Winter 1994-95, S. 231-248.

Felderer, B./Homburg, St. (1994): Makroökonomik und neue Makroökonomik, 6. Auflage, Berlin u.a.

Finanzwissenschaftlicher Beirat beim Bundesministerium für Wirtschaft und Finanzen (1972): Gutachten zur Finanzierung eines höheren Staatsanteils am Sozialprodukt, Bonn.

Fischer-Menshausen, H. (1968): Mittelfristige Finanzplanung im Bundesstaat, in: Kaiser, J.H. (Hrsg.): Planung III, Baden-Baden, S. 73-109.

Franz, W. (1985): Challenges to the German Economy 1973-1983. Supply Shocks, Investment Slowdown, Inflation Variability and the Underutilization of Labor, in: Zeitschrift für Wirtschafts- und Sozialwissenschaft, 105. Jg., S. 407-430. 
Franz, W. (1987): Hysteresis, persistence, and the NAIRU: an empirical analysis for the Federal Republic of Germany, in: Layard, R./Calmfors, L. (Eds.): The Fight against Unemployment, Cambridge u.a.

Franz, W. (1994): Arbeitsökonomik, Berlin u.a.

Franz, W./König, H. (1986): The Nature and Causes of Unemployment in the Federal Republic of Germany since the 1970s: An Empirical Investigation, in: Economica, Vol. 53, Supplement, S. S219-S244.

Friedman, B.M. (1988): Day of Reckoning. The Consequences of American Economic Policy Under Reagan and After, New York.

Friedman, M. (1968): The Role of Monetary Policy, in: American Economic Review, Vol. 58, S. 1-17.

Friedman, M. (1970): Die optimale Geldmenge und andere Essays, München.

Gandenberger, O. (1981): Theorie der öffentlichen Verschuldung, in: Handbuch der Finanzwissenschaft, 3. Auflage, Band III, Tübingen.

Gebauer, W. (1982): Realzins, Inflation und Kapitalzins. Eine Neuinterpretation des Fisher-Theorems, Berlin u.a.

Glastetter, W./Paulert, R./Spörel, U. (1983): Die wirtschaftliche Entwicklung in der Bundesrepublik Deutschland 1950-1980. Befunde, Aspekte, Hintergründe, Frankfurt/Main.

Grassinger, R. (1993): Verfestigte Arbeitslosigkeit. Das Hysteresis-Phänomen unter besonderer Berücksichtigung des Humankapitalansatzes, Beiträge zur Arbeitsmarkt- und Berufsforschung 174 (Institut für Arbeitsmarkt- und Berufsforschung der Bundesanstalt für Arbeit), Nürnberg.

Gresser, K. (1974): Probleme der mehrjährigen öffentlichen Finanzplanung, Berlin.

Grund, W. (1968): Die mehrjährige Finanzplanung des Bundes. Grundkonzeption, Methoden und ihre Problematik, in: Kaiser, J.H. (Hrsg.): Planung III, Baden-Baden, S. 47-72.

Gruppe Politikinformation am Forschungsschwerpunkt Arbeitsmarkt und Beschäftigung, WZB (1987): Internationale Chronik zur Arbeitsmarktpolitik 1980-1986, in: Auer, P./Bruche, G./Kühl, J. (Hrsg.): Chronik zur Arbeitsmarktpolitik. National 1978-1986. International 1980-1986, Beiträge zur Arbeitsmarkt- und Berufsforschung 99 (Institut für Arbeitsmarkt- und Berufsforschung der Bundesanstalt für Arbeit), Nürnberg, S. 253-583.

Gschwendter, H. (1981): Staatsverschuldung und finanzwirtschaftlicher Ausgabenspielraum, in: Finanzarchiv, N.F. Bd. 39, S. 306-318. 


\section{Literatur}

Haan, J. de/Sterks, C.G.M./Kam, C.A. de (1992): Towards budget discipline: an economic assessment of the possibilities for reducing national deficits in the run-up to EMU, Economic Papers (Europäische Kommission), No. 99, Dezember 1992.

Haan, J. de/Zelhorst, D. (1992): Positive theories of public debt. Some evidence for Germany, in: Verbon, H./Winden, F. van (Hrsg.): The Political Economy of Government Debt, Amsterdam.

Haliassos, M./Tobin, J. (1990): The Macroeconomics of Government Finance, in: Friedman, B.M./Hahn, F.H. (Hrsg.): Handbook of Monetary Economics, Amsterdam u.a.

Hamilton, J.D./Flavin, M.A. (1986): On the Limitations of Government Borrowing: A Framework for Empirical Testing, in: American Economic Review, Vol. 76, No. 4, S. 808-819.

Hansen, H.-J. (1991): Keynesianische oder Klassische Arbeitslosigkeit in der Bundesrepublik Deutschland. Empirische Analysen mit einem ökonometrischen Ungleichgewichtsmodell, Idstein.

Hansmeyer, K.-H. (1973a): Das Gesetz zur Förderung der Stabilität und des Wachstums der Wirtschaft (I), in: WISU 6/73, S. 279-284.

Hansmeyer, K.-H. (1973b): Das Gesetz zur Förderung der Stabilität und des Wachstums der Wirtschaft (II), in: WISU 7/73, S. 331-335.

Hansmeyer, K.-H. (1973c): Das Gesetz zur Förderung der Stabilität und des Wachstums der Wirtschaft (III), in: WISU 8/73, S. 379-382.

Hansmeyer, K.-H. (1977): Erfahrungen mit dem Stabilitätsgesetz, in: Wirtschaftsdienst, 57. Jg., S. 607-612.

Hansmeyer, K.-H. (1984): Der öffentliche Kredit I. Der Staat als Schuldner, 3. Aufl., Frankfurt/Main

Hardes, H.-D. (1974): Einkommenspolitik in der BRD. Stabilität und Gruppeninteressen: Der Fall Konzertierte Aktion, Frankfurt/Main

Heinemann, F. (1993): Sustainability of National Debt in Europe, in: Intereconomics, März/April 1993, S. 61-68.

Herr, H. (1987): Die Sozialdemokraten und das Schuldenmachen, in: Die Neue Gesellschaft, Frankfurter Hefte 12, Dezember 1987.

Holtz-Eakin, D./Schwartz, A.E. (1995): Infrastructure in a structural model of economic growth, in: Regional Science and Urban Economics, Vol. 25, S. 131-151.

Horn, G./Möller, J. (1985): Keynesianische oder Klassische Arbeitslosigkeit in der Bundesrepublik Deutschland?, in: Ifo-Studien, 31. Jg., S. 203-238. 
Hujer, R./Hansen, H.-J./Klein, E. (1989): Zeitvariable Parameter in ökonometrischen Modellen, in: WISU 7/89, S. 423-428.

Hujer, R./Hansen, H.-J./Klein, E. (1994): Der Beitrag makroökonometrischer Modelle zur Beurteilung wirtschafts- und sozialpolitischer Maßnahmen - Erfahrungen mit dem Frankfurter Modell, in: Deutsche Forschungsgemeinschaft (Hrsg.): Mikroanalytische Grundlagen der Gesellschaftspolitik, Band 1, Ausgewählte Probleme und Lösungsansätze, Berlin, S. 176-196.

Institut „Finanzen und Steuern“ e.V. (1982): Die mittelfristige Finanzplanung des Bundes in der Zeit von 1971 bis 1982, Grüner Brief Nr. 216, Bonn, September 1982, Bearbeiter: Manfred Schlick.

Institut „Finanzen und Steuern“ e.V. (1993): Konvergenzkriterien einer Europäischen Währungsunion: Zur Logik der Bestimmungen von Maastricht, Grüner Brief Nr. 317, Bonn, Mai 1993, Bearbeiter: Hagen Lesch.

Institut „Finanzen und Steuern“ e.V. (1995): Zur Eigendynamik unserer Staatsverschuldung, Grüner Brief Nr. 333, Bonn, Januar 1995, Bearbeiter: Ludwig Dornbusch.

Issing, O. (1992): Einführung in die Geldpolitik, 4. Auflage, München.

Jahreswirtschaftsbericht 1970 der Bundesregierung, Bonn.

Jahreswirtschaftsbericht 1971 der Bundesregierung, Bonn. Jahreswirtschaftsbericht 1972 der Bundesregierung, Bonn. Jahreswirtschaftsbericht 1973 der Bundesregierung, Bonn. Jahreswirtschaftsbericht 1974 der Bundesregierung, Bonn. Jahreswirtschaftsbericht 1975 der Bundesregierung, Bonn. Jahreswirtschaftsbericht 1976 der Bundesregierung, Bonn. Jahreswirtschaftsbericht 1977 der Bundesregierung, Bonn. Jahreswirtschaftsbericht 1978 der Bundesregierung, Bonn. Jahreswirtschaftsbericht 1979 der Bundesregierung, Bonn. Jahreswirtschaftsbericht 1980 der Bundesregierung, Bonn. Jahreswirtschaftsbericht 1981 der Bundesregierung, Bonn. Jahreswirtschaftsbericht 1982 der Bundesregierung, Bonn. Jahreswirtschaftsbericht 1983 der Bundesregierung, Bonn. Jarchow, H.-J./Rühmann, P. (1994): Monetäre Außenwirtschaft, I. Monetäre Außenwirtschaftstheorie, 4. Auflage, Göttingen.

Joines, D.H. (1991): How Large A Federal Budget Deficit Can We Sustain?, in: Contemporary Policy Issues, Vol. IX, Juli 1991.

Kalmbach, P. (Hrsg.)(1973): Der neue Monetarismus, München. 
Kitterer, W. (1988): Staatsverschuldung und intertemporale Allokation, in: Jahrbuch für Nationalökonomie und Statistik, Bd. 204, S. 346-363.

Kitterer, W. (1993): Staatsverschuldung und Haushaltskonsolidierung - Folgen für den Standort Deutschland, in: Wirtschaftsdienst, 73. Jg., S. 633638.

Klein, F. (1969): Finanz- und Haushaltsreform. Gesetzestexte, München, Wien.

Klein, M./Neumann M.J.M. (1990): Seigniorage: What Is It and Who Gets It?, in: Weltwirtschaftliches Archiv, Bd. 126, S. 205-221.

Klementa, J. (1972): Das Verhältnis von Planung und Budgetierung in der Bundesrepublik Deutschland, in: Kaiser, J.H. (Hrsg.): Planung VI, BadenBaden, S. 69-77.

Kloten, N. (1976): Erfolg und Mißerfolg der Stabilisierungspolitik (19691974), in: Deutsche Bundesbank (Hrsg.): Währung und Wirtschaft in Deutschland 1876-1975, Frankfurt/Main, S. 643-690.

Kock, H. (1975): Stabilitätspolitik im föderalistischen System der Bundesrepublik Deutschland, Köln.

Kock, H./Leifert, E./Schmid, A./Stirnberg, L. (1977): Konzepte der Konjunktursteuerung. Von der globalen zur strukturellen Orientierung, Köln.

König, H./Entorf, H. (1990): Strukturelle Arbeitslosigkeit und unausgelastete Kapazitäten: Ergebnisse eines makroökonomischen Rationierungsmodells, in: Allgemeines Statistisches Archiv, Bd. 74, S. 117-136.

König, H./Zimmermann, K.F. (1986): Determinants of Employment Policy of German Manufacturing Firms: A Survey-Based Evaluation, in: Oppenländer, K.H./Poser, G. (Hrsg.): Business Cycle Surveys in the Assessment of Economic Activity, Aldershot, S. 631-649.

Kohl, H. (1982): Koalition der Mitte: Für eine Politik der Erneuerung. Regierungserklärung des Bundeskanzlers vor dem Deutschen Bundestag am 13. Oktober 1982, abgedruckt in: Bulletin Nr. 93, S. 853-868.

Kotlikoff, L. J. (1993): From Deficit Delusion to the Fiscal Balance Rule: Looking for an Economically Meaningful Way to Assess Fiscal Policy, in: Journal of Economics, Supplement 17-41.

Kromphardt, J. (1987): Arbeitslosigkeit und Inflation, eine Einführung in die makroökonomischen Kontroversen, Göttingen.

Kühl, J. (1982): Das Arbeitsförderungsgesetz (AFG) von 1969. Grundzüge seiner arbeitsmarkt- und beschäftigungspolitischen Konzeption, in: Mitteilungen aus der Arbeitsmarkt- und Berufsforschung, 15. Jg., S. 251-260. 
Kühl, J. (1987): Nationale Chronik zur Arbeitsmarktpolitik 1978-1986, in: Auer, P./Bruche, G./Kühl, J. (Hrsg.): Chronik zur Arbeitsmarktpolitik. National 1978-1986. International 1980-1986, Beiträge zur Arbeitsmarktund Berufsforschung 99 (Institut für Arbeitsmarkt- und Berufsforschung der Bundesanstalt für Arbeit), Nürnberg, S. 3-252.

Lambsdorff, O. Graf (1981): Wachsende Staatsverschuldung - Wohltat oder Plage?, in: Simmert, D.B./Wager, K.-D. (Hrsg.): Staatsverschuldung kontrovers, Schriftenreihe der Bundeszentrale für Politische Bildung, Bd. 174, Bonn, S. 197-202.

Lampert, H. (1989): 20 Jahre Arbeitsförderungsgesetz, in: Mitteilungen aus der Arbeitsmarkt- und Berufsforschung, 22. Jg., S. 173-186.

Laroque, G. (1989): Comparative Estimates of a Macroeconomic Disequilibrium Model. France, Germany, the U.K. and the U.S.A., in: European Economic Review, Vol. 33, S. 963-995.

Layard, R./Nickell, St./Jackman, R. (1994): Unemployment. Macroeconomic Performance and the Labour Market, New York.

Lehment, H. (1988): Fiskalpolitik in offenen Volkswirtschaften, Kieler Studien Nr. 219, Tübingen.

Leibfritz, W./Roseveare, D./Noord, P. van den (1994): Fiscal Policy, Government Debt and Economic Performance, OECD Working Papers, No. 33, Paris.

Leicht, A. (1970): Die Haushaltsreform, München, Wien.

Licht, G./Steiner, V. (1991): Abgang aus der Arbeitslosigkeit, Individualeffekte und Hysteresis. Eine Panelanalyse für die Bundesrepublik Deutschland, in: Helberger, Ch./Bellmann, L./Blaschke, D. (Hrsg.): Erwerbstätigkeit und Arbeitslosigkeit. Analysen auf der Grundlage des sozio-ökonomischen Panels, Beiträge zur Arbeitsmarkt- und Berufsforschung 144 (Institut für Arbeitsmarkt- und Berufsforschung der Bundesanstalt für Arbeit), S. 182-206.

Ludvigson, S. (1996): The macroeconomic effects of government debt in a stochastic growth model, in: Journal of Monetary Economics, Vol. 38, S. 25-45.

Mackscheidt, K./Steinhausen, J. (1978): Finanzpolitik I. Grundfragen fiskalpolitischer Lenkung, Tübingen.

Maussner, A. (1985): Stabilisierungspolitik im Lichte von Gleichgewichtsund Ungleichgewichtstheorie, Göttingen. 
McCallum, B.T. (1984): Are Bond-financed Deficits Inflationary? A Ricardian Analysis, in: Journal of Political Economy, Vol. 92, S. 123-135.

Möller, A. (1969): Kommentar zum Gesetz zur Förderung der Stabilität und des Wachstums der Wirtschaft, 2. Auflage, Hannover.

Möller, J. (1982): Strukturelle Veränderungen von Arbeitsmarktrelationen. Theoretische und empirische Aspekte, Frankfurt/Main.

Nachtkamp, H.-H. (1976): Mehrjährige Finanzplanungen und mittelfristige Zielprojektionen der Bundesregierung. Über Unzulänglichkeiten des Planungskalküls und die Einordnung der Stabilisierungspolitik des Bundes in die mittelfristige Wirtschaftsplanung, Baden-Baden.

Neldner, M. (1984): Seigniorage, inflationäre Staatshaushaltsfinanzierung und das Problem der optimalen Geldmenge, in: WISU 8-9, S. 411-418.

Neumark, F. (1971): Ermessensfreiheit oder Automatismus, Zürich.

Niskanen, W.A. (1988): Reaganomics. An Insider's Account of the Policies and the People, New York, Oxford.

Oberhauser, A. (1975): Stabilitätspolitik bei steigender Staatsquote, Schriften der Kommission für wirtschaftlichen und sozialen Wandel, Bd. 43, Göttingen.

Oberhauser, A. (1985): Das Schuldenparadox. Paradoxical Effects of Public Debt, in: Jahrbücher für Nationalökonomie und Statistik, Vol. 4, Bd. 200, S. 333-348.

Oberhauser, A. (1996): Mehr Arbeitslosigkeit durch Parallelpolitik: eine Folge einzelwirtschaftlichen Denkens, in: Wirtschaftsdienst, Heft XI, S. 566573.

OECD (1990): OECD Economic Outlook, 48, Dezember 1990.

OECD (1996): OECD Economic Outlook, 60, Dezember 1996.

Ohndorf, W. (1992): Das arbeitsmarktpolitische Programm der Bundesregierung für Regionen mit besonderen Beschäftigungsproblemen vom Mai 1979 (Sonderprogramm '79) - Anregungen für die neuen Bundesländer -, in: Mitteilungen aus der Arbeitsmarkt- und Berufsforschung, 25. Jg., S. 303-313.

Osterwald, E. (1982): Die Entstehung des Stabilitätsgesetzes, Frankfurt/ Main, New York.

Pasinetti, L.L. (1997): The Social 'Burden' Of High Interest Rates, in: Arestis, P./Palma, G./Sawver, M. (Ed.): Capital Controversy, Post-Keynesian Economics And The History Of Economic Thought. 
Pfadt, R./Schröder, J. (1994): Die Bonität hoch verschuldeter Länder und die europäische Währungsunion, in: Rübel, G. (Hrsg.): Perspektiven der Europäischen Integration, Heidelberg, S. 177-208.

Pfeiffer, A. (1984): Ergebnisse und Aussichten der Wirtschaftspolitik nach einem Jahr Regierung Kohl/Genscher, in: Gewerkschaftliche Monatshefte, 35. Jg., S. 146-157.

Phelps, E.S. (1970): Microeconomic Foundation of Employment and Inflation Theory, London and Basingstoke.

Recktenwald, H.C. (Hrsg.)(1970): Kosten-Nutzen-Analyse und Programmbudget. Grundlage staatlicher Entscheidung und Planung, Tübingen.

Reineke, U. (1986), Segmentationstheorien des Arbeitsmarktes und Beschäftigungsniveau. Eine Auseinandersetzung mit der theoretischen und empirischen Relevanz der Entkopplungshypothese, Bern, New York.

Rothschild, K.W. (1981): Einleitung in die Ungleichgewichtstheorie, Berlin.

Roubini, N./Sachs, J.D. (1989): Political and Economic Determinants of Budget Deficits in the Industrial Democracies, in: European Economic Review, Vol. 33, S. 903-938.

Rürup, B. (1971): Die Programmfunktion des Bundeshaushaltsplanes. Die deutsche Haushaltsreform im Lichte der amerikanischen Erfahrungen mit dem Planning-Programming-Budgeting System, Berlin.

Rürup, B. (1982): Begrenzungskriterien der staatlichen Kreditaufnahme, in: Bombach, G./Gahlen, B./Ott, A.E. (Hrsg.): Möglichkeiten und Grenzen der Staatstätigkeit, Tübingen.

Rürup, B./Körner, H. (1985): Finanzwissenschaft. Grundlagen der öffentlichen Finanzwirtschaft, 2. Auflage, Düsseldorf.

Rürup, B./Siedenberg, A. (1974): Das Stabilitätsgesetz im Spiegel der Kritik, in: Konjunkturpolitik, 20. Jg., S. 1-19.

Sachverständigenrat zur Begutachtung der gesamtwirtschaftlichen Entwicklung (1968): Alternativen außenwirtschaftlicher Anpassung, Jahresgutachten $1968 / 69$.

Sachverständigenrat zur Begutachtung der gesamtwirtschaftlichen Entwicklung (1969): Im Sog des Booms, Jahresgutachten 1969/70.

Sachverständigenrat zur Begutachtung der gesamtwirtschaftlichen Entwicklung (1970): Konjunktur im Umbruch - Risiken und Chancen -, Jahresgutachten 1970/71. 
Sachverständigenrat zur Begutachtung der gesamtwirtschaftlichen Entwicklung (1971): Währung, Geldwert, Wettbewerb - Entscheidungen für morgen -, Jahresgutachten 1971/72.

Sachverständigenrat zur Begutachtung der gesamtwirtschaftlichen Entwicklung (1972): Gleicher Rang für den Geldwert, Jahresgutachten 1972/73.

Sachverständigenrat zur Begutachtung der gesamtwirtschaftlichen Entwicklung (1973): Mut zur Stabilisierung, Jahresgutachten 1973/74.

Sachverständigenrat zur Begutachtung der gesamtwirtschaftlichen Entwicklung (1974): Vollbeschäftigung für morgen, Jahresgutachten 1974/75.

Sachverständigenrat zur Begutachtung der gesamtwirtschaftlichen Entwicklung (1975): Vor dem Aufschwung, Jahresgutachten 1975/76.

Sachverständigenrat zur Begutachtung der gesamtwirtschaftlichen Entwicklung (1976): Zeit zum Investieren, Jahresgutachten 1976/77.

Sachverständigenrat zur Begutachtung der gesamtwirtschaftlichen Entwicklung (1977): Mehr Wachstum - Mehr Beschäftigung, Jahresgutachten 1977/78.

Sachverständigenrat zur Begutachtung der gesamtwirtschaftlichen Entwicklung (1978): Wachstum und Währung, Jahresgutachten 1978/79.

Sachverständigenrat zur Begutachtung der gesamtwirtschaftlichen Entwicklung (1979): Herausforderung von außen, Jahresgutachten 1979/80.

Sachverständigenrat zur Begutachtung der gesamtwirtschaftlichen Entwicklung (1980): Unter Anpassungszwang, Jahresgutachten 1980/81.

Sachverstăndigenrat zur Begutachtung der gesamtwirtschaftlichen Entwicklung (1981): Investieren für mehr Beschäftigung, Jahresgutachten 1981/82.

Sachverständigenrat zur Begutachtung der gesamtwirtschaftlichen Entwicklung (1982): Gegen Pessimismus, Jahresgutachten 1982/83.

Sachverständigenrat zur Begutachtung der gesamtwirtschaftlichen Entwicklung (1995): Im Standortwettbewerb, Jahresgutachten 1995/96.

Sachverständigenrat zur Begutachtung der gesamtwirtschaftlichen Entwicklung (1996): Reformen voranbringen, Jahresgutachten 1996/97.

Sargent, Th. J./Wallace, N. (1986): Some Unpleasant Monetarist Arithmetic, in: Sargent, Th. J.: Rational Expectations and Inflation, New York u.a. Scharpf, F.W. (1974): Politische Durchsetzbarkeit innerer Reformen, Schriften der Kommission für wirtschaftlichen und sozialen Wandel, Bd. 28, Göttingen. 
Scharpf, F.W./Garlichs, D./Maier, F./Maier, H. (1982): Implementationsprobleme offensiver Arbeitsmarktpolitik. Das Sonderprogramm der Bundesregierung für Regionen mit besonderen Beschäftigungsproblemen, Frankfurt.

Scherf, H. (1986): Enttäuschte Hoffnungen - vergebene Chancen. Die Wirtschaftspolitik der Sozial-liberalen Koalition 1969-1982, Göttingen.

Schlecht, O. (1968): Gesamtwirtschaftliche Zielprojektionen als Grundlage der wirtschaftspolitischen Planung in der Marktwirtschaft, in: Kaiser, J.H. (Hrsg.): Planung III, Baden-Baden, S. 111-127.

Schlesinger, H./Weber, M./Ziebarth, G. (1993): Staatsverschuldung - ohne Ende? Zur Rationalität und Problematik des öffentlichen Kredits, Darmstadt.

Schlömann, H. (1972): Planungssysteme und Organisation der öffentlichen Verwaltung in der Bundesrepublik Deutschland, in: Kaiser, J.H. (Hrsg.): Planung VI, Baden-Baden, S. 531-544.

Schmidt, J. (1977): Zur Bedeutung der Staatsausgaben für die Beschäftigung. Input-Output-Studie der Beschäftigungswirkungen von Ausgabenkürzungen, Deutsches Institut für Wirtschaftsforschung, Beiträge zur Strukturforschung, Heft 46, Berlin.

Schmidt, K./Wille, E. (1970): Die mehrjährige Finanzplanung. Wunsch und Wirklichkeit, Tübingen.

Schneider, J. (1987): Marktfehler und Arbeitslosigkeit, Regensburg.

Sneessens, H.R./Drèze, J.H. (1986): A Discussion of Belgian Unemployment, Combining Traditional Concepts and Disequilibrium Econometrics, in: Economica, Vol. 53, Supplement, S. S89-S119.

Spahn, H.-P. (1988): Bundesbank und Wirtschaftskrise, Regensburg.

Spaventa, L. (1987): The Growth of Public Debt, in: IMF Staff Papers, 34, S. 374-399.

Statistisches Bundesamt (1994a): Fachserie 14, Finanzen und Steuern, Reihe 5, Schulden der öffentlichen Haushalte, Stuttgart.

Statistisches Bundesamt (1994b): Fachserie 14, Finanzen und Steuern, Reihe

3.1, Rechnungsergebnisse des öffentlichen Gesamthaushalts, Stuttgart.

Statistisches Bundesamt (1995): Fachserie 14, Finanzen und Steuern, Reihe 5, Schulden der öffentlichen Haushalte, Stuttgart.

Stern, K./Münch, P./Hansmeyer, K.-H. (1972): Gesetz zur Förderung der Stabilität und des Wachstums der Wirtschaft. Kommentar, 2. Auflage, Stuttgart u.a. 
Stützel, W. (1978): Ober- und Untergrenzen der öffentlichen Verschuldung, in: Kredit und Kapital, 11. Jg., S. 429-450.

Tobin, J. (1984): Budget Deficits, Federal Debt, And Inflation, in: Sommers, A.T. (Ed.): Reconstructing the Federal Budget, New York u.a.

Webber, D. (1982): Zwischen programmatischem Anspruch und politischer Praxis: Die Entwicklung der Arbeitsmarktpolitik in der Bundesrepublik Deutschland von 1974 bis 1982, in: Mitteilungen aus der Arbeitsmarktund Berufsforschung, 15. Jg., S. 261-275.

Werner, H. (1987): Arbeitsmarktpolitische Maßnahmen in den Ländern der Europäischen Gemeinschaft und in Schweden - eine Übersicht, in: Mitteilungen aus der Arbeitsmarkt- und Berufsforschung, 20. Jg., S. 441-452.

Wilcox, D.W. (1989): The Sustainability of Government Deficits: Implications of the Present-Value Borrowing Constraint, in: Journal of Money, Credit, and Banking, Vol. 21, No. 3, August 1989, S. 291-306.

Witterauf, P. (1983): Beschäftigungspolitik heute - Ein internationaler Vergleich der Depressionsbekämpfung in den siebziger Jahren, Volkswirtschaftliche Studien, Band 18: Wirtschaftspolitik, Gelsenkirchen.

Zunker, A. (1972): Finanzplanung und Bundeshaushalt. Zur Koordinierung und Kontrolle durch den Bundesfinanzminister, Frankfurt/Main, Berlin. 


\section{FINANZWISSENSCHAFTLICHE SCHRIFTEN}

Band 1 Werner Steden: Finanzpolitik und Einkommensverteilung. Ein Wachstums- und Konjunkturmodell der Bundesrepublik Deutschland. 1979.

Band 2 Rainer Hagemann: Kommunale Finanzplanung im föderativen Staat. 1976.

Band 3 Klaus Scherer: Maßstäbe zur Beurteilung von konjunkturellen Wirkungen des öffentlichen Haushalts. 1977.

Band 4 Brita Steinbach: "Formula Flexibility" - Kritische Analyse und Vergleich mit diskretionärer Konjunkturpolitik. 1977.

Band 5 Hans-Georg Petersen: Personelle Einkommensbesteuerung und Inflation. Eine theoretisch-empirische Analyse der Lohn- und veranlagten Einkommensteuer in der Bundesrepublik Deutschland. 1977.

Band 6 Friedemann Tetsch: Raumwirkungen des Finanzsystems der Bundesrepublik Deutschland. Eine Untersuchung der Auswirkungen der Finanzreform von 1969 auf die Einnahmenposition der untergeordneten Gebietskörperschaften und ihrer regionalpolitischen Zieladäquanz. 1978.

Band 7 Wilhelm Pfähler: Normative Theorie der fiskalischen Besteuerung. Ein methodologischer und theoretischer Beitrag zur Integration der normativen Besteuerungstheorie in der Wohlfahrtstheorie. 1978.

Band 8 Wolfgang Wiegard: Optimale Schattenpreise und Produktionsprogramme für öffentliche Unternehmen. Second-Best Modelle im finanzwirtschaftlichen Staatsbereich. 1978.

Band 9 Hans P. Fischer: Die Finanzierung des Umweltschutzes im Rahmen einer rationalen Umweltpolitik. 1978.

Band 10 Rainer Paulenz: Der Einsatz finanzpolitischer Instrumente in der Forschungs- und Entwicklungspolitik. 1978.

Band 11 Hans-Joachim Hauser: Verteilungswirkungen der Staatsverschuldung. Eine kreislauftheoretische Inzidenzbetrachtung. 1979.

Band 12 Gunnar Schwarting: Kommunale Investitionen. Theoretische und empirische Untersuchungen der Bestimmungsgründe kommunaler Investitionstätigkeit in NordrheinWestfalen 1965-1972. 1979.

Band 13 Hans-Joachim Conrad: Stadt-Umland-Wanderung und Finanzwirtschaft der Kernstädte. Amerikanische Erfahrungen, grundsätzliche Zusammenhänge und eine Fallstudie für das Ballungsgebiet Frankfurt am Main. 1980.

Band 14 Cay Folkers: Vermögensverteilung und staatliche Aktivität. Zur Theorie distributiver Prozesse im Interventionsstaat. 1981.

Band 15 Helmut Fischer: US-amerikanische Exportförderung durch die DISC-Gesetzgebung. 1981.

Band 16 Günter Ott: Einkommensumverteilungen in der gesetzlichen Krankenversicherung. Eine quantitative Analyse. 1981.

Band 17 Johann Hermann von Oehsen: Optimale Besteuerung. (Optimal Taxation). 1982.

Band 18 Richard Kössler: Sozialversicherungsprinzip und Staatszuschüsse in der gesetzlichen Rentenversicherung. 1982.

Band 19 Hinrich Steffen: Zum Handlungs- und Entscheidungsspielraum der kommunalen Investitionspolitik in der Bundesrepublik Deutschland. 1983.

Band 20 Manfred Scheuer: Wirkungen einer Auslandsverschuldung des Staates bei flexiblen Wechselkursen. 1983. 
Band 21 Christian Schiller: Staatsausgaben und crowding-out-Effekte. Zur Effizienz einer Finanzpolitik keynesianischer Provenienz. 1983.

Band 22 Hannelore Weck: Schattenwirtschaft: Eine Möglichkeit zur Einschränkung der öffentlichen Verwaltung? Eine ökonomische Analyse. 1983.

Band 23 Wolfgang Schmitt: Steuern als Mittel der Einkommenspolitik. Eine Ergänzung der Stabilitätspolitik? 1984.

Band 24 Wolfgang Laux: Erhöhung staatswirtschaftlicher Effizienz durch budgetäre Selbstbeschränkung? Zur Idee einer verfassungsmäßig verankerten Ausgabengrenze. 1984.

Band 25 Brita Steinbach-van der Veen: Steuerinzidenz. Methodologische Grundlagen und empirisch-statistische Probleme von Länderstudien. 1985.

Band 26 Albert Peters: Ökonomische Kriterien für eine Aufgabenverteilung in der Marktwirtschaft. Eine deskriptive und normative Betrachtung für den Allokationsbereich. 1985.

Band 27 Achim Zeidler: Möglichkeiten zur Fortsetzung der Gemeindefinanzreform. Eine theoretische und empirische Analyse. 1985.

Band 28 Peter Bartsch: Zur Theorie der längerfristigen Wirkungen 'expansiver' Fiskalpolitik. Eine dynamische Analyse unter besonderer Berücksichtigung der staatlichen Budgetbeschränkung und ausgewählter Möglichkeiten der öffentlichen Defizittinanzierung. 1986.

Band 29 Konrad Beiwinkel: Wehrgerechtigkeit als finanzpolitisches Verteilungsproblem. Möglichkeiten einer Kompensation von Wehrungerechtigkeit durch monetäre Transfers. 1986.

Band 30 Wolfgang Kitterer: Effizienz- und Verteilungswirkungen des Steuersystems. 1986.

Band 31 Heinz Dieter Hessler: Theorie und Politik der Personalsteuern. Eine Kritik ihrer Einkommens- und Vermögensbegriffe mit Blick auf die Leistungsfähigkeitstheorie. 1994.

Band 32 Wolfgang Scherf: Die beschäftigungspolitische und fiskalische Problematik der Arbeitgeberbeiträge zur Rentenversicherung. Eine Auseinandersetzung mit der Kritik an der lohnbezogenen Beitragsbemessung. 1987.

Band 33 Andreas Mästle: Die Steuerunion. Probleme der Harmonisierung spezifischer Gütersteuern. 1987.

Band 34 Günter Ott: Internationale Verteilungswirkungen im Finanzausgleich der Europäischen Gemeinschaften. 1987.

Band 35 Heinz Haller: Zur Frage der zweckmäßigen Gestalt gemeindlicher Steuern. Ein Diskussionsbeitrag zur Gemeindesteuerreform. 1987.

Band 36 Thomas Kuhn: Schlüsselzuweisungen und fiskalische Ungleichheit. Eine theoretische Analyse der Verteilung von Schlüsselzuweisungen an Kommunen. 1988.

Band 37 Walter Hahn: Steuerpolitische Willensbildungsprozesse in der Europäischen Gemeinschaft. Das Beispiel der Umsatzssteuer-Harmonisierung. 1988.

Band 38 Ulrike Hardt: Kommunale Finanzkraft. Die Problematik einer objektiven Bestimmung kommunaler Einnahmemöglichkeiten in der gemeindlichen Haushaltsplanung und im kommunalen Finanzausgleich. 1988.

Band 39 Jochen Michaelis: Optimale Finanzpolitik im Modell überlappender Generationen. 1989.

Band 40 Bernd Raffelhüschen: Anreizwirkungen der sozialen Alterssicherung. Eine dynamische Simulationsanalyse. 1989.

Band 41 Berend Diekmann: Die Anleihe- und Darlehenstransaktionen der Europäischen Gemeinschaften. 1990.

Band 42 Helmut Kaiser: Konsumnachfrage, Arbeitsangebot und optimale Haushaltsbesteuerung. Theoretische Ergebnisse und mikroökonometrische Simulation für die Bundesrepublik Deutschland. 1990. 
Band 43 Rüdiger von Kleist: Das Gramm-Rudman-Hollings-Gesetz. Ein gescheiterter Versuch der Haushaltskonsolidierung. 1991.

Band 44 Rolf Hagedorn: Steuerhinterziehung und Finanzpolitik. Ein theoretischer Beitrag unter besonderer Berücksichtigung der Hinterziehung von Zinserträgen. 1991.

Band 45 Cornelia S. Behrens: Intertemporale Verteilungswirkungen in der gesetzlichen Krankenversicherung der Bundesrepublik Deutschland. 1991.

Band 46 Peter Saile: Ein ökonomischer Ansatz der Theorie der intermediären Finanzgewalten Die Kirchen als Parafisci. 1992.

Band 47 Peter Gottfried: Die verdeckten Effizienzwirkungen der Umsatzsteuer. Eine empirische allgemeine Gleichgewichtsanalyse. 1992.

Band 48 Andreas Burger: Umweltorientierte Beschäftigungsprogramme. Eine Effizienzanalyse am Beispiel des "Sondervermögens Arbeit und Umwelt". 1992.

Band 49 Jeanette Malchow: Die Zuordnung verteilungspolitischer Kompetenzen in der Europäischen Gemeinschaft. Eine Untersuchung aufgrund einer Fortentwicklung der ökonomischen Theorie des Föderalismus. 1992.

Band 50 Barbara Seidel: Die Einbindung der Bundesrepublik Deutschland in die Europäischen Gemeinschaften als Problem des Finanzausgleichs. 1992.

Band 51 Ralph Wiechers: Markt und Macht im Rundfunk. Zur Stellung der öffentlich-rechtlichen Rundfunkanstalten im dualen Rundfunksystem der Bundesrepublik Deutschland. 1992.

Band 52 Klaus Eckhardt: Probleme einer Umweltpolitik mit Abgaben. 1993.

Band 53 Oliver Schwarzkopf: Die Problematik unterschiedlicher Körperschaftsteuersysteme innerhalb der EG. 1993.

Band 54 Thorsten Giersch: Bergson-Wohlfahrtsfunktion und normative Ökonomie. 1993.

Band 55 Li-Fang Chou: Selbstbeteiligung bei Arzneimitteln aus ordnungspolitischer Sicht. Das Beispiel der Bundesrepublik Deutschland. 1993.

Band 56 Harald Schlee: Einkommensteuerliche Behandlung von Transferzahlungen. Zur Neuordnung der Familienbesteuerung sowie der Besteuerung von Versicherungsleistungen und Sozialtransfers. 1994.

Band 57 Alexander Spermann: Kommunales Krisenmanagement. Reaktionen baden-württembergischer Stadtkreise auf steigende Sozialhilfekosten und Einnahmenausfälle (198092). 1993.

Band 58 Otto Roloff / Sibylle Brander / Ingo Barens / Claudia Wesselbaum: Direktinvestitionen und internationale Steuerkonkurrenz. 1994.

Band 59 Claudia Wesselbaum-Neugebauer: Internationale Steuerbelastungsvergleiche. 1994.

Band 60 Stephanie Miera: Kommunales Finanzsystem und Bevölkerungsentwicklung. Eine Analyse des kommunalen Finanzsystems vor dem Hintergrund der sich abzeichnenden Bevölkerungsentwicklung am Beispiel Niedersachsens unter besonderer Berücksichtigung des Landkreises Wolfenbüttel und seiner Gemeinden. 1994.

Band 61 Wolfgang Schert: Die Bedeutung des kaldorianischen Verteilungsmechanismus für die gesamtwirtschaftlichen Wirkungen der staatlichen Neuverschuldung. 1994.

Band 62 Rainer Volk: Vergleich der Vergünstigungseffekte der verschiedenen investitionsfördernden Maßnahmen. 1994.

Band 63 Hans-Georg Napp: Kommunale Finanzautonomie und ihre Bedeutung für eine effiziente lokale Finanzwirtschaft. 1994. 2., unveränderte Auflage 1994.

Band 64 Bernd Rahmann / Uwe Steinborn / Günter Vornholz: Empirische Analyse der Autonomie lokaler Finanzwirtschaften in der Europäischen Gemeinschaft. 1994. 
Band 65 Carsten Kühl: Strategien zur Finanzierung der Altlastensanierung. 1994.

Band 66 Stephan Boll: Intergenerationale Umverteilungswirkungen der Fiskalpolitik in der Bundesrepublik Deutschland. Ein Ansatz mit Hilfe des Generational Accounting. 1994.

Band 67 Karl Justus Bernhard Neumärker: Finanzverfassung und Staatsgewalt in der Demokratie. Ein Beitrag zur konstitutionellen Finanztheorie. 1995.

Band 68 Christian Haslbeck: Zentrale versus dezentrale Internalisierung externer Effekte bei unvollständiger Information. 1995.

Band 69 Regina Müller: Horizontale oder vertikale Transfers zur Durchsetzung eines horizontalen Finanzausgleichs. 1995.

Band 70 Christian Hockenjos: Öffentliche Sportförderung in der Bundesrepublik Deutschland. Darstellung und finanztheoretische Analyse. 1995.

Band 71 Manfred Rosenstock: Die Kontrolle und Harmonisierung nationaler Beihilfen durch die Kommission der Europäischen Gemeinschaften. 1995.

Band 72 Christian Rüsch: Wohnungsbau- und Wohneigentumspolitik im Rahmen der Einkommensteuer. Eine Analyse unter steuersystematischen, verteilungspolitischen und fiskalischen Aspekten. 1996.

Band 73 Stephan Winters: Die kollektive Vorsorge für den Pflegefall im Alter. Eine Untersuchung am Beispiel der gesetzlichen Pflegeversicherung in den Niederlanden. 1996.

Band 74 Knut Blind: Allokationsineffizienzen auf Sicherheitsmärkten: Ursachen und Lösungsmöglichkeiten. Fallstudie: Informationssicherheit in Kommunikationssystemen. 1996.

Band 75 Barbara Petrick-Rump: Ökonomische Wirkungen von Steueramnestien. Untersuchung konkreter Erfahrungen ausgewählter Länder mit dem Einsatz von Steueramnestien anhand eines effizienten Steueramnestieprogramms. 1996.

Band 76 Georg Hirte: Effizienzwirkungen von Finanzausgleichsregelungen. Eine Empirische Allgemeine Gleichgewichtsanalyse für die Bundesrepublik Deutschland. 1996.

Band 77 Ulrike Kirchhoff: Die rheinland-pfälzischen Gemeinden im System des Finanzausgleichs. 1996.

Band 78 Kerstin Keil: Der soziale Mietwohnungsbau: Mängel und Alternativen. 1996.

Band 79 Bernhard Manzke: Kinderlastenausgleich versus verstärkte Einwanderung. Alternative Ansätze zur langfristigen Sicherung der Gesetzlichen Rentenversicherung. 1997.

Band 80 Hariolf M. Wenzler: Institutionenökonomik und öffentliche Finanzkontrolle. Eine Analyse am Beispiel der Europäischen Union. 1997.

Band 81 Joachim Nagel: Supply-Side Policy in den USA. Eine theoretische und empirische Analyse der angebotsorientierten Wirtschaftspolitik Reagans unter besonderer Berücksichtigung steuerlicher Aspekte. 1997.

Band 82 Heinz Lampert: Krise und Reform des Sozialstaates. 1997.

Band 83 Monika Hanswillemenke / Bernd Rahmann: Zwischen Reformen und Verantwortung für Vollbeschäftigung. Die Finanz- und Haushaltspolitik der sozial-liberalen Koalition von 1969 bis 1982. 1997. 


\title{
Prinzipien wirtschaftlichen Handelns und ihre Anwendung
}

\section{Umriß einer Wirtschaftsphilosophie}

\author{
Frankfurt/M., Berlin, Bern, New York, Paris, Wien, 1996. 145 S., 1 Abb.
}

ISBN 3-631-30477-3 • br. DM 48.-*

Die enge Verbindung von Wirtschaft und Weltgeschehen verlangt nach einer exakten Untersuchung ihrer Notwendigkeit, Möglichkeiten und Grenzen. Die Ökonomie wird auf ihre erlernbaren theoretischen Prinzipien, die Möglichkeiten ihrer Anwendung und die Bedingungen ihrer praktischen Durchsetzbarkeit hin untersucht. Ergebnis ist eine gründliche Neubestimmung des Verhältnisses von Theorie, Anwendung und Praxis. Die historisch bekannten Wirtschaftsschulen und -richtungen werden auf ihren Geltungsanspruch hinterfragt. Politische und ideologische Rahmenbedingungen werden in ihrem Einfluß auf Privat- und Gemeinwirtschaft erläutert und Modelle zur Überprüfung von Wirtschaftspolitik und Wirtschaftsethik aufgezeigt.

Aus dem Inhalt: Wissenschaftsanspruch, Definition und Methoden der Ökonomie - Einfluß von politischen und ideologischen Rahmenbedingungen . Privat- und Gemeinwirtschaft - Wirtschaftsschulen und Wirtschaftsmodelle . Wirtschaftsrecht und Wirtschaftsethik - Ökonomische Begriffsbildung und Wissenschaft · Ökonomie und Philosophie

Frankfurt/M - Berlin - Bern - New York - Paris - Wien

Auslieferung: Verlag Peter Lang AG

Jupiterstr. 15, CH-3000 Bern 15

Telefax (004131) 9402131

*inklusive Mehrwertsteuer

Preisänderungen vorbehalten 